\title{
Development of Perfluoroarene:Arene Interactions for Macrocyclic En-Yne Metathesis and the Total Synthesis of Macrocyclic Natural Products.
}

Table of contents

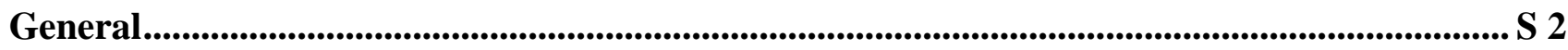

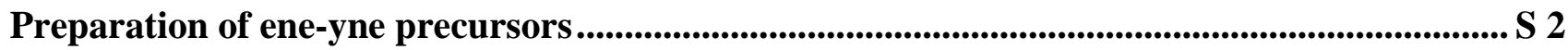

General procedure for alkylations via the Mitsunobu reaction. ...............................................S 5

General procedure for the direct ene-yne metathesis reaction................................................. S 15

General procedure for the hydrogenation of the ene-yne metathesis products ..................... S 21

Results of MP2 energies and DFT calculations...............................................S22

NMR spectra of all precursors and metathesis products...................................................... S 24 


\section{SUPPORTING INFORMATION}

\section{General}

All reactions that were carried out under anhydrous conditions were performed under an inert argon or nitrogen atmosphere in glassware that had previously been dried overnight at $120^{\circ} \mathrm{C}$ or had been flame dried and cooled under a stream of argon or nitrogen ${ }^{1}$. Isolated yields reflect the mass obtained following flash column silica gel chromatography using the method reported by W. C. Still ${ }^{2}$ and using silica gel (40-63 nm; 230-240 mesh). Analytical thin-layer chromatography (TLC) was performed on aluminum-backed silica gel 60 coated with a fluorescence indicator $\left(0.25 \mathrm{~mm}, \mathrm{~F}_{254}\right.$. $)$. All the compounds were UV active, and no development of the TLCs was required. Visualization of TLC plate was performed by UV $(254 \mathrm{~nm})$. All mixed solvent eluents are reported as v/v solutions. Concentration refers to removal of volatiles at low pressure on a rotary evaporator. All reported compounds were homogeneous by thin layer chromatography (TLC) and by ${ }^{1} \mathrm{H}$ NMR. NMR spectra were taken in deuterated $\mathrm{CDCl}_{3}$.Signals due to the solvent served as the internal standard. The acquisition parameters are shown on all spectra. The ${ }^{1} \mathrm{H}$ NMR chemical shifts and coupling constants were determined assuming first-order behavior. Multiplicity is indicated by one or more of the following: s (singlet), $\mathrm{d}$ (doublet), $\mathrm{t}$ (triplet), $\mathrm{q}$ (quartet), $\mathrm{m}$ (multiplet), br (broad); the list of couplings constants $(J)$ corresponds to the order of the multiplicity assignment. The ${ }^{1} \mathrm{H}$ NMR assignments were made based on chemical shift and multiplicity and were confirmed, where necessary, by homonuclear decoupling, 2D COSY experiments. The ${ }^{13} \mathrm{C} N M R$ assignments were made on the basis of chemical shift and multiplicity and were confirmed, where necessary, by two dimensional H/C correlation experiments (HSQC). High resolution mass spectroscopy (HRMS) was done using ESI mode of ionization. Charged molecular ion $[\mathrm{M}+\mathrm{H}]^{+}$and $[\mathrm{M}+\mathrm{Na}]^{+}$data are reported.

\section{Preparation of ene-yne precursors}

A general procedure for the preparation of precursors 7A- 10B-12A-14B- 16A- 18B- 20A- 22B24A- 26B- 28A- 30B-32A-34B- 36B- 38- 40A- 42B is detailed below. Following this description, the spectral data is indicated for each individual compound. Note that in general, we do not observe any ${ }^{13} \mathrm{C} N \mathrm{NR}$ signals for the five fluorine-bearing carbon atoms of the pentafluorobenzyl auxiliary or for the $\mathrm{CF}_{3}$ carbon. In select cases where a different synthetic route was necessary, these procedures are described in detail.

Ene-Yne precursors 7A- 10B- 12A- 14B- 16A- 18B- 20A- 22B- 32A-34B- 36B- 40A- 42B were prepared following this sequence:

${ }^{1}$ D. F. Shriver, M. A. Drezdon, The Manipulation of Air-Sensitive Compounds; $2^{\text {nd }}$ Edition, ed.; Wiley: New York, 1986.

${ }^{2}$ Still, W. C.; Kahn, M.; Mitra, A. J. Org. Chem. 1978, 43, 2923. 


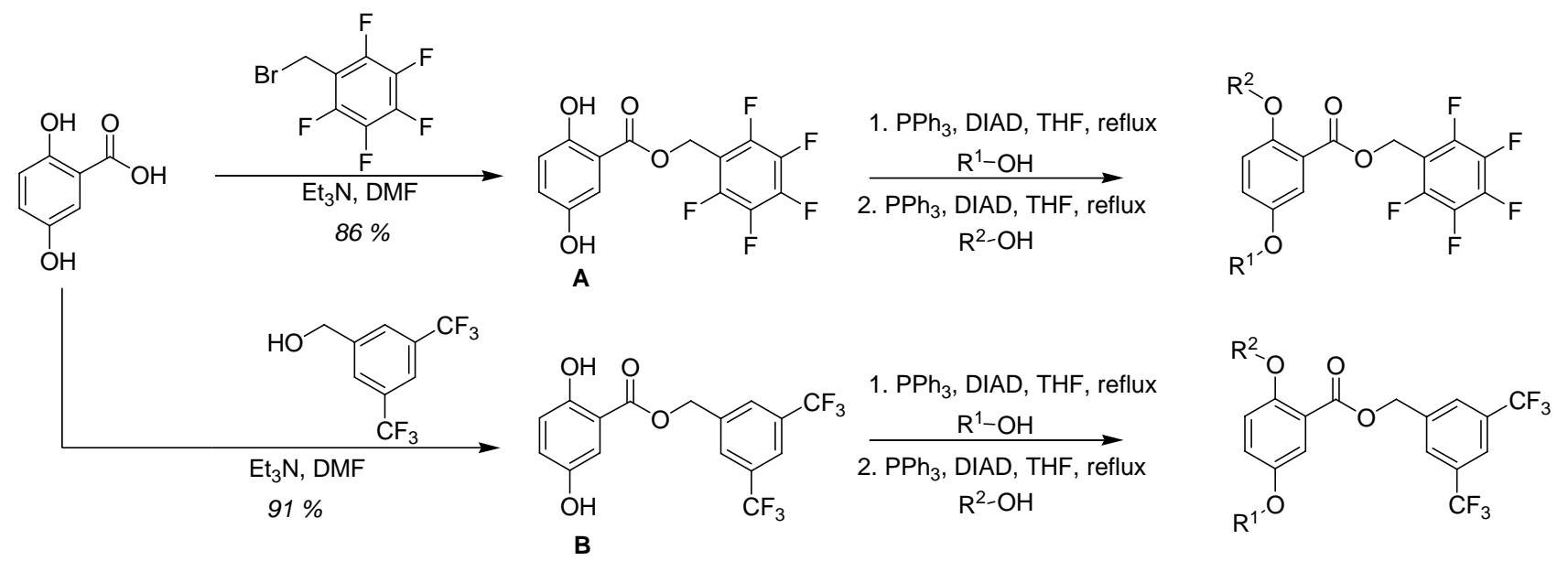

Figure SI1: Preparation of metathesis precursors 7A- 10B- 12A- 14B- 16A- 18B- 20A- 22B- 32A34B- 36B- 40A and 42B.

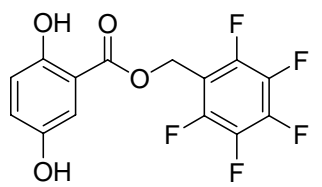

A

In a round bottom flask, 2, 5-dihydroxybenzoic acid (154 $\mathrm{mg}, 1.00 \mathrm{mmol}, 1.0$ equiv.) is dissolved at room temperature in dry DMF and triethylamine $(0.140 \mathrm{~mL}, 1.0 \mathrm{mmol}, 1.0$ equiv.) is added drop wise, the reaction is exothermic. Then, pentafluorobenzyl bromide $(0.140 \mathrm{~mL}, 1.0 \mathrm{mmol}, 1.0$ equiv.) is added to the mixture. The reaction was monitored by TLC (Hexanes: Ethyl acetate 7:1) and was typically complete after 1 hour, although reactions could be left overnight at room temperature. The DMF is then evaporated under vacuum, the reaction mixture was dry-packed and purified by flash column silica chromatography using (Hexanes: Ethyl acetate 7:1) to afford the product as a colorless solid (287 mg, $86 \%)$.

${ }^{1} \mathbf{H}$ NMR $\left(\mathrm{CDCl}_{3}, 400 \mathrm{MHz}\right) \delta 10.07(\mathrm{~s}, 1 \mathrm{H}), 7.21(\mathrm{~d}, J=3.1 \mathrm{~Hz}, 1 \mathrm{H}), 7.03(\mathrm{dd}, J=9.0,3.1 \mathrm{~Hz}$, $1 \mathrm{H}), 6.90(\mathrm{~d}, J=9.0 \mathrm{~Hz}, 1 \mathrm{H}), 5.46(\mathrm{~s}, 2 \mathrm{H}), 4.56(\mathrm{~s}, 1 \mathrm{H}), ;{ }^{13} \mathrm{C} \mathbf{~ N M R}\left(\mathrm{CDCl}_{3}, 100 \mathrm{MHz}\right) \delta 168.8$, 156.1, 147.7, 124.7, 118.7, 114.6, 111.3, 54.0; HRMS (ES) Calculated for $\mathrm{C}_{14} \mathrm{H}_{8} \mathrm{~F}_{5} \mathrm{O}_{4}[\mathrm{M}+\mathrm{H}]^{+}$: 335.0337 found 335.0342 .

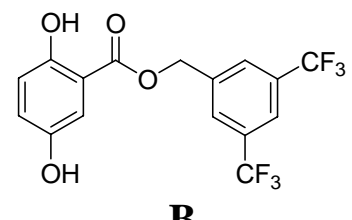

B

In a round bottom flask, 2, 5-dihydroxybenzoic acid (154 mg, $1.00 \mathrm{mmol}, 1.0$ equiv.) is dissolved at room temperature in dry DMF and triethylamine $(0.140 \mathrm{~mL}, 1.0 \mathrm{mmol}, 1.0$ equiv.) is added drop wise, the reaction is exothermic. Then, 3,5-di(trifluoromethyl)benzyl bromide $(310 \mathrm{mg}, 1.0 \mathrm{mmol}$, 1.0 equiv.) is added to the mixture. The reaction was monitored by TLC (Hexanes: Ethyl acetate 7:1) and was typically complete after 1 hour, although reaction could be left overnight at room temperature. The DMF is then evaporated under vacuum, the reaction mixture was dry-packed and 
purified by flash column silica chromatography using (Hexanes: Ethyl acetate 7:1) to afford the product as a colorless solid (346 mg, $91 \%$ ).

${ }^{1} \mathrm{H} \mathrm{NMR}\left(\mathrm{CDCl}_{3}, 400 \mathrm{MHz}\right) \delta 10.17(\mathrm{~s}, 1 \mathrm{H}), 7.89(\mathrm{~s}, 3 \mathrm{H}), 7.30(\mathrm{~d}, J=3.1 \mathrm{~Hz}, 1 \mathrm{H}), 7.05(\mathrm{dd}, J=9.1$, $3.1 \mathrm{~Hz}, 1 \mathrm{H}), 6.90(\mathrm{~d}, J=9.1 \mathrm{~Hz}, 1 \mathrm{H}), 5.45(\mathrm{~s}, 2 \mathrm{H}), 4.82(\mathrm{~s}, 1 \mathrm{H}) ;{ }^{13} \mathrm{C} \mathrm{NMR}\left(\mathrm{CDCl}_{3}, 100 \mathrm{MHz}\right) \delta$ 169.2, 156.1, 147.8, 137.7, 132.1 (q, $\mathrm{CF}_{3}$ ), 128.4, 124.8, 122.6, 121.7, 118.8, 114.5, 111.4, 65.3; HRMS (ES, negative mode of ionization) Calculated for $\mathrm{C}_{16} \mathrm{H}_{9} \mathrm{~F}_{6} \mathrm{O}_{4}[\mathrm{M}-\mathrm{H}]^{-}: 379.0405$ found 379.0423 .

Ene-Yne precursor 28A was prepared following this sequence:<smiles>O=C(O)c1cc(O)c(C(=O)O)cc1O</smiles>

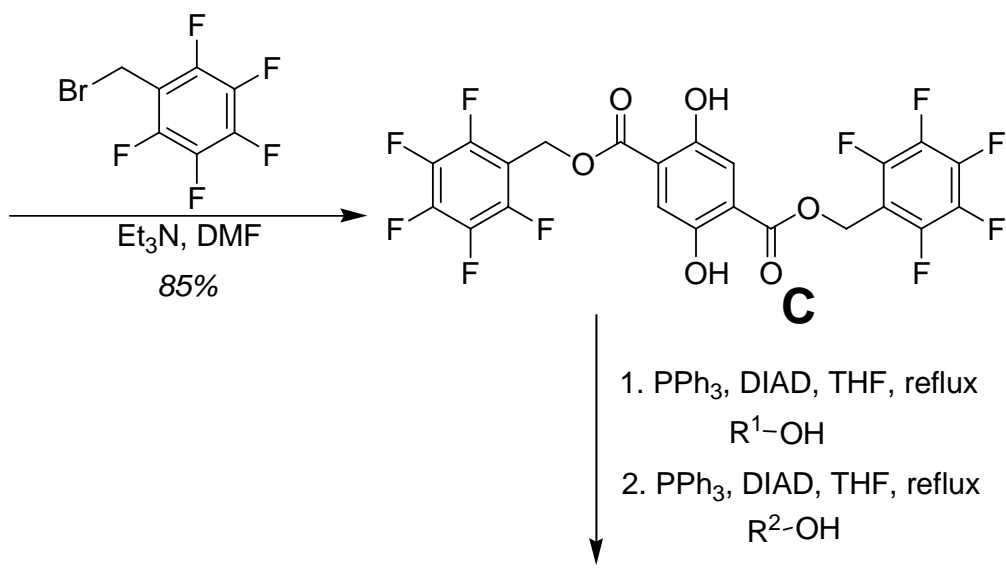<smiles>[R]Oc1cc(C(=O)OCc2c(F)c(F)c(F)c(F)c2F)c(O[R])cc1C(=O)OCc1c(F)c(F)c(F)c(F)c1F</smiles>

Figure SI2: Preparation of metathesis precursor 28A.<smiles>O=C(OCc1c(F)c(F)c(F)c(F)c1F)c1cc(O)c(C(=O)OCc2c(F)c(F)c(F)c(F)c2F)cc1O</smiles>

C

In a round bottom flask, 2,5-dihydroxy-terephtalic acid (198 mg, $1.0 \mathrm{mmol}, 1.0$ equiv.) is dissolved at room temperature in dry DMF. Triethylamine $(0.280 \mathrm{~mL}, 2.0 \mathrm{mmol}, 2.0$ equiv. $)$ is added drop wise. The reaction is exothermic. Then, pentafluorobenzyl bromide $(0.280 \mathrm{~mL}, 2.0 \mathrm{mmol}, 2.0$ equiv.) is added to the mixture. The reaction was monitored by TLC (Hexanes: Ethyl acetate 4:1) and was typically complete after 1 hour, although reactions could be left overnight at room temperature. The DMF is then evaporated under vacuum, the reaction mixture was dry-packed and purified by flash column silica chromatography using (Hexanes: Ethyl acetate 7:1) to afford the product as a colorless solid (475 $\mathrm{mg}, 85 \%)$.

${ }^{\mathbf{1}} \mathbf{H}$ NMR $\left(\mathrm{CDCl}_{3}, 400 \mathrm{MHz}\right) \delta 9.79(\mathrm{~s}, 2 \mathrm{H}), 7.41(\mathrm{~s}, 2 \mathrm{H}), 5.48(\mathrm{~s}, 4 \mathrm{H}) ;{ }^{13} \mathbf{C} \mathbf{~ N M R}\left(\mathrm{CDCl}_{3}, 100 \mathrm{MHz}\right)$ $\delta$ 168.1, 153.0, 118.0, 117.9, 54.5; HRMS (ES) Calculated for $\mathrm{C}_{22} \mathrm{H}_{9} \mathrm{~F}_{10} \mathrm{O}_{6}[\mathrm{M}+\mathrm{H}]^{+}: 559.0239$ found 559.0226 . 
Ene-Yne precursor 24A was prepared following this sequence:

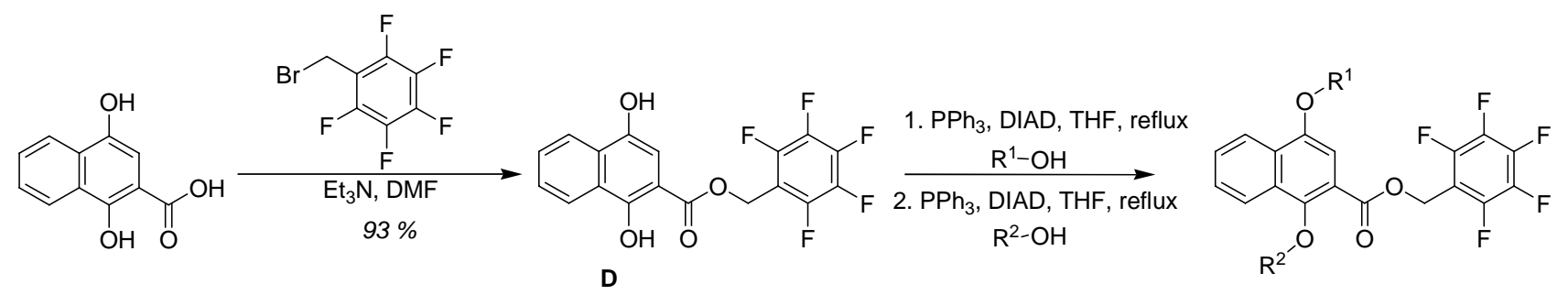

Figure SI3: Preparation of metathesis precursor 24A

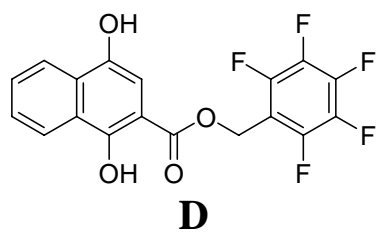

In a round bottom flask, 1, 4-dihydroxy-2-naphthanoic acid (204 mg, $1.0 \mathrm{mmol}, 1.0$ equiv.) is dissolved at room temperature in dry DMF and triethylamine $(0.140 \mathrm{~mL}, 1.0 \mathrm{mmol}, 1.0$ equiv. $)$ is added drop wise, the reaction is exothermic. Then, pentafluorobenzyl bromide $(0.140 \mathrm{~mL}, 1.0 \mathrm{mmol}$, 1.0 equiv.) is added to the mixture.The reaction was monitored by TLC (Hexanes: Ethyl acetate 7:1) and was typically complete after 1 hour, although reactions could be left overnight at room temperature. The DMF is then evaporated under vacuum, the reaction mixture was dry-packed and purified by flash column silica chromatography using (Hexanes: Ethyl acetate 7:1) to afford the product as a colourless solid (358 $\mathrm{mg}, 93 \%)$.

${ }^{1} \mathbf{H}$ NMR $\left(\mathrm{CDCl}_{3}, 400 \mathrm{MHz}\right) \delta 11.30(\mathrm{~s}, 1 \mathrm{H}), 8.39(\mathrm{~d}, J=8.3 \mathrm{~Hz}, 1 \mathrm{H}), 8.11(\mathrm{~d}, J=8.3 \mathrm{~Hz}, 1 \mathrm{H}), 7.67$ $(\mathrm{t}, J=7.8 \mathrm{~Hz}, 1 \mathrm{H}), 7.58(\mathrm{t}, J=7.2 \mathrm{~Hz}, 1 \mathrm{H}), 7.00(\mathrm{~s}, 1 \mathrm{H}), 5.50(\mathrm{~s}, 2 \mathrm{H}), 4.92(\mathrm{~s}, 1 \mathrm{H}) ;{ }^{13} \mathbf{C}$ NMR $\left(\mathrm{CDCl}_{3}, 100 \mathrm{MHz}\right) \delta 169.7,156.2,143.3,129.4,129.1,126.6,125.5,124.1,121.7,104.7,103.5$, 53.9; HRMS (ES, negative mode of ionization) Calculated for $\mathrm{C}_{18} \mathrm{H}_{8} \mathrm{~F}_{5} \mathrm{O}_{4}[\mathrm{M}-\mathrm{H}]$ : 383.0342 found 383.0351 .

\section{General procedure for alkylations via the Mitsunobu reaction.}

In a round bottom flask, pentafluorobenzyl-2, 5-dihydroxybenzoate (1.0 eq.) was added to triphenylphosphine (1.2 eq.) and the alkenyl or alkynyl alcohol (0.5 to 0.6 eq. $)^{3}$. This mixture was then dissolved in dry THF and heated to reflux. DIAD (1.2 eq.) was then added drop wise and the reaction was left under reflux and monitored by TLC. Typically after 10-15 hours the reaction was complete and no starting material was observed by TLC. The reaction mixture was then concentrated, dry-packed and purified by flash column silica chromatography using (Hexanes: Ethyl acetate 20:1). Isolated yields were obtained in the range of 55-90\%. The products were immediately carried onto the second alkylation. In a round bottom flask, the mono-alkylated product (1.0 eq.) was added to triphenylphosphine (1.6 eq.) and the alkenyl or alkynyl alcohol (1.2 eq.). This mixture was then dissolved in dry THF and heated to reflux. While under reflux, DIAD (1.6 eq.) was added drop wise and the reaction was left under reflux and monitored by TLC. After 15 hours, the reaction

\footnotetext{
${ }^{3}$ This stochiometry is important to avoid the formation of dialkylated product.
} 
mixture was concentrated, dry-packed and purified by flash chromatography using (Hexanes: Ethyl acetate 10:1). Isolated yields of approximately $60 \%$ were typically obtained.

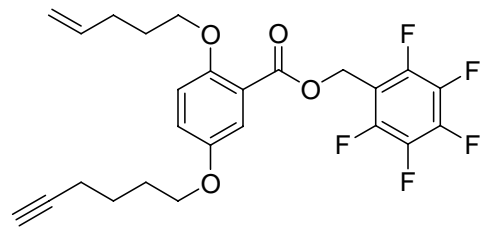

7A

${ }^{1} \mathbf{H}$ NMR $\left(\mathrm{CDCl}_{3}, 400 \mathrm{MHz}\right) \delta 7.29(\mathrm{~d}, J=3.0 \mathrm{~Hz}, 1 \mathrm{H}), 7.00(\mathrm{dd}, J=9.0,3.2 \mathrm{~Hz}, 1 \mathrm{H}), 6.85(\mathrm{~d}, J=$ $9.1 \mathrm{~Hz}, 1 \mathrm{H}), 5.82(\mathrm{~m}, 1 \mathrm{H}), 5.39(\mathrm{~s}, 2 \mathrm{H}), 5.06-4.97(\mathrm{~m}, 2 \mathrm{H}), 3.97-3.86(\mathrm{~m}, 4 \mathrm{H}), 2.24-2.20(\mathrm{~m}, 4 \mathrm{H})$, $1.95(\mathrm{t}, J=2.6 \mathrm{~Hz}, 1 \mathrm{H}), 1.87-1.83(\mathrm{~m}, 4 \mathrm{H}), 1.66-1.63(\mathrm{~m}, 2 \mathrm{H}) ;{ }^{13} \mathbf{C ~ N M R}\left(\mathrm{CDCl}_{3}, 100 \mathrm{MHz}\right) \delta$ 165.5, 153.0, 152.4, 146.9, 120.6, 119.6, 116.8, 115.1, 115.0, 84.0, 68.8, 68.6, 68.0, 53.6, 29.8, 28.3, 28.2, 24.9, 18.1; HRMS (ES) Calculated for $\mathrm{C}_{25} \mathrm{H}_{24} \mathrm{~F}_{5} \mathrm{O}_{4}[\mathrm{M}+\mathrm{H}]^{+}: 483.1589$ found 483.1591. Calculated for $\mathrm{C}_{25} \mathrm{H}_{23} \mathrm{~F}_{5} \mathrm{O}_{4} \mathrm{Na}[\mathrm{M}+\mathrm{Na}]^{+}$: 505.1409, found 505.1470.

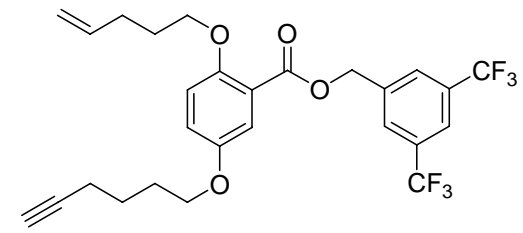

10B

${ }^{1} \mathbf{H}$ NMR $\left(\mathrm{CDCl}_{3}, 400 \mathrm{MHz}\right) \delta 7.92(\mathrm{~s}, 2 \mathrm{H}), 7.85(\mathrm{~s}, 1 \mathrm{H}), 7.35(\mathrm{~d}, J=3.1 \mathrm{~Hz}, 1 \mathrm{H}), 7.03(\mathrm{dd}, J=9.1$, $3.1 \mathrm{~Hz}, 1 \mathrm{H}), 6.91(\mathrm{~d}, J=9.1 \mathrm{~Hz}, 1 \mathrm{H}), 5.75(\mathrm{~m}, 1 \mathrm{H}), 5.44(\mathrm{~s}, 2 \mathrm{H}), 4.99-4.92(\mathrm{~m}, 2 \mathrm{H}), 4.00-3.94(\mathrm{~m}$, 4H), 2.27- $2.24(\mathrm{~m}, 2 \mathrm{H}), 1.17-1.12(\mathrm{~m}, 2 \mathrm{H}), 1.97(\mathrm{t}, J=2.6 \mathrm{~Hz}, 1 \mathrm{H}), 1.92-1.69(\mathrm{~m}, 4 \mathrm{H}), 1.80-1.60$ $(\mathrm{m}, 2 \mathrm{H}) ;{ }^{13} \mathbf{C} \mathbf{N M R}\left(\mathrm{CDCl}_{3}, 100 \mathrm{MHz}\right) \delta 165.9,153.0,152.4,138.8,137.5,131.9\left(\mathrm{q}, \mathrm{CF}_{3}\right), 128.0$, $127.2,124.5,122.0,121.8,120.8,119.7,116.7,115.1,115.0,84.0,68.8,68.6,67.9,64.9,29.8,28.2$, 24.9, 18.1; HRMS (ES) Calculated for $\mathrm{C}_{27} \mathrm{H}_{27} \mathrm{~F}_{6} \mathrm{O}_{4}[\mathrm{M}+\mathrm{H}]^{+}: 529.1808$ found 529.1811. Calculated for $\mathrm{C}_{27} \mathrm{H}_{26} \mathrm{~F}_{5} \mathrm{O}_{4} \mathrm{Na}[\mathrm{M}+\mathrm{Na}]^{+}:$551.1627, found 551.1632.

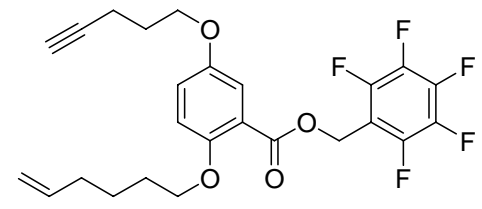

$12 \mathrm{~A}$

${ }^{1} \mathbf{H}$ NMR $\left(\mathrm{CDCl}_{3}, 400 \mathrm{MHz}\right) \delta 7.31(\mathrm{~d}, J=3.1 \mathrm{~Hz}, 1 \mathrm{H}), 7.00(\mathrm{dd}, J=9.0,3.1 \mathrm{~Hz}, 1 \mathrm{H}), 6.87(\mathrm{~d}, J=$ $9.0 \mathrm{~Hz}, 1 \mathrm{H}), 5.85(\mathrm{~m}, 1 \mathrm{H}), 5.39(\mathrm{~s}, 2 \mathrm{H}), 5.02-4.94(\mathrm{~m}, 2 \mathrm{H}), 4.02(\mathrm{t}, J=6.1 \mathrm{~Hz}, 2 \mathrm{H}), 3.94(\mathrm{t}, J=6.5$ $\mathrm{Hz}, 2 \mathrm{H}), 2.40-2.36(\mathrm{~m}, 2 \mathrm{H}), 2.40-2.36(\mathrm{~m}, 2 \mathrm{H}), 2.03-2.10(\mathrm{~m}, 2 \mathrm{H}), 2.00-1.94(\mathrm{~m}, 3 \mathrm{H}), 1.76-1.70$ $(\mathrm{m}, 2 \mathrm{H}), 1.52-1.48(\mathrm{~m}, 2 \mathrm{H}) ;{ }^{13} \mathrm{C} \mathbf{N M R}\left(\mathrm{CDCl}_{3}, 100 \mathrm{MHz}\right) \delta 165.5,153.2,152.1,138.4,120.6$, 119.6, 116.9, 114.9, 114.6, 83.3, 69.4, 68.9, 66.7, 53.6, 33.3, 28.6, 28.1, 25.1, 15.1; HRMS (ES) Calculated for $\mathrm{C}_{25} \mathrm{H}_{24} \mathrm{~F}_{5} \mathrm{O}_{4}[\mathrm{M}+\mathrm{H}]^{+}: 483.1589$ found 483.1592. Calculated for $\mathrm{C}_{25} \mathrm{H}_{23} \mathrm{~F}_{5} \mathrm{O}_{4} \mathrm{Na}[\mathrm{M}+$ $\mathrm{Na}]^{+}:$505.1409, found 505.1408. 


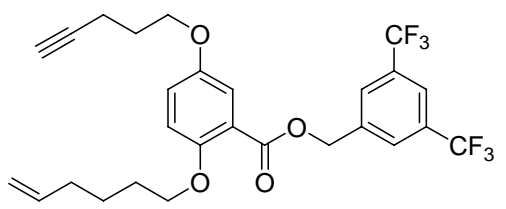

14B

${ }^{1} \mathbf{H}$ NMR $\left(\mathrm{CDCl}_{3}, 400 \mathrm{MHz}\right) \delta 7.92(\mathrm{~s}, 2 \mathrm{H}), 7.85(\mathrm{~s}, 1 \mathrm{H}), 7.37(\mathrm{~d}, J=3.2 \mathrm{~Hz}, 1 \mathrm{H}), 7.05(\mathrm{dd}, J=9.1$, $3.2 \mathrm{~Hz}, 1 \mathrm{H}), 6.91(\mathrm{~d}, J=9.1 \mathrm{~Hz}, 1 \mathrm{H}), 5.75(\mathrm{~m}, 1 \mathrm{H}), 5.44(\mathrm{~s}, 2 \mathrm{H}), 4.98-4.90(\mathrm{~m}, 2 \mathrm{H}), 4.05(\mathrm{t}, J=6.1$ $\mathrm{Hz}, 2 \mathrm{H}), 3.99(\mathrm{t}, J=6.6 \mathrm{~Hz}, 2 \mathrm{H}), 2.41-2.37(\mathrm{~m}, 2 \mathrm{H}), 2.04-1.96(\mathrm{~m}, 5 \mathrm{H}), 1.77-1.73(\mathrm{~m}, 2 \mathrm{H}), 1.53-$ $1.48(\mathrm{~m}, 2 \mathrm{H}) ;{ }^{13} \mathrm{C}$ NMR $\left(\mathrm{CDCl}_{3}, 100 \mathrm{MHz}\right) \delta 165.9,153.1,152.2,138.8,138.4,132.0\left(\mathrm{q}, \mathrm{CF}_{3}\right)$, 128.0, 127.2, 121.8, 120.8, 119.8, 115.0, 114.7, 121.8, 120.8, 83.3, 69.4, 68.9, 66.8, 64.9, 33.2, 28.5, 28.1, 25.1, 15.1; HRMS (ES) Calculated for $\mathrm{C}_{27} \mathrm{H}_{27} \mathrm{~F}_{6} \mathrm{O}_{4}[\mathrm{M}+\mathrm{H}]^{+}$: 529.1808 found 529.1803. Calculated for $\mathrm{C}_{27} \mathrm{H}_{26} \mathrm{~F}_{6} \mathrm{O}_{4} \mathrm{Na}[\mathrm{M}+\mathrm{Na}]^{+}:$551.1627, found 551.1627.

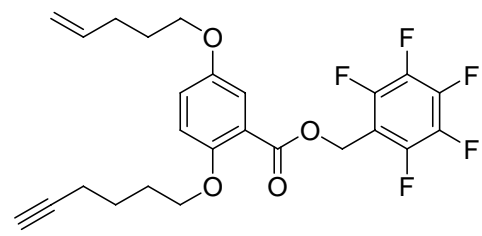

16A

${ }^{1} \mathbf{H}$ NMR $\left(\mathrm{CDCl}_{3}, 400 \mathrm{MHz}\right) \delta 7.29(\mathrm{~d}, J=3.0 \mathrm{~Hz}, 1 \mathrm{H}), 7.00(\mathrm{dd}, J=9.0,3.2 \mathrm{~Hz}, 1 \mathrm{H}), 6.85(\mathrm{~d}, J=$ $9.1 \mathrm{~Hz}, 1 \mathrm{H}), 5.82(\mathrm{~m}, 1 \mathrm{H}), 5.39(\mathrm{~s}, 2 \mathrm{H}), 5.06-4.97(\mathrm{~m}, 2 \mathrm{H}), 3.97-3.86(\mathrm{~m}, 4 \mathrm{H}), 2.24-2.20(\mathrm{~m}, 4 \mathrm{H})$, $1.95(\mathrm{t}, J=2.6 \mathrm{~Hz}, 1 \mathrm{H}), 1.87-1.83(\mathrm{~m}, 4 \mathrm{H}), 1.66-1.63(\mathrm{~m}, 2 \mathrm{H}) ;{ }^{13} \mathbf{C ~ N M R}\left(\mathrm{CDCl}_{3}, 100 \mathrm{MHz}\right) \delta$ $165.5,152.9,152.3,137.6,120.6,119.5,116.9,115.1,114.8,83.9,68.8,68.5,67.0,53.5,30.0,28.3$, 28.2, 24.8, 17.9; HRMS (ES) Calculated for $\mathrm{C}_{25} \mathrm{H}_{24} \mathrm{~F}_{5} \mathrm{O}_{4}[\mathrm{M}+\mathrm{H}]^{+}: 483.1589$ found 483.1597. Calculated for $\mathrm{C}_{25} \mathrm{H}_{23} \mathrm{~F}_{5} \mathrm{O}_{4} \mathrm{Na}[\mathrm{M}+\mathrm{Na}]^{+}:$505.1409, found 505.1483.

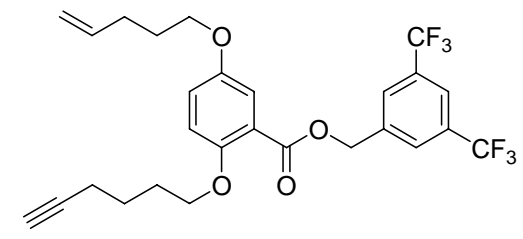

$18 B$

${ }^{1} \mathbf{H}$ NMR $\left(\mathrm{CDCl}_{3}, 400 \mathrm{MHz}\right) \delta 7.94(\mathrm{~s}, 2 \mathrm{H}), 7.87(\mathrm{~s}, 1 \mathrm{H}), 7.37(\mathrm{~d}, J=3.1 \mathrm{~Hz}, 1 \mathrm{H}), 7.06(\mathrm{dd}, J=9.1$, $3.1 \mathrm{~Hz}, 1 \mathrm{H}), 6.93(\mathrm{~d}, J=9.1 \mathrm{~Hz}, 1 \mathrm{H}), 5.85(\mathrm{~m}, 1 \mathrm{H}), 5.46(\mathrm{~s}, 2 \mathrm{H}), 5.09-5.02(\mathrm{~m}, 2 \mathrm{H}), 4.02(\mathrm{t}, J=6.0$ $\mathrm{Hz}, 2 \mathrm{H}), 3.96(\mathrm{t}, J=6.4 \mathrm{~Hz}, 2 \mathrm{H}), 2.27-2.20(\mathrm{~m}, 4 \mathrm{H}), 1.98-1.86(\mathrm{~m}, 5 \mathrm{H}), 1.68-1.64(\mathrm{~m}, 2 \mathrm{H}) ;{ }^{13} \mathrm{C}$ NMR $\left(\mathrm{CDCl}_{3}, 100 \mathrm{MHz}\right) \delta 166.0,152.9,152.5,138.8,137.7,131.8\left(\mathrm{q}, \mathrm{CF}_{3}\right), 128.0,127.2,124.5$, $122.0,122.1,121.6,121.8,120.8,119.7,116.8,115.2,114.9,83.9,68.9,68.7,67.8,64.9,30.0,28.4$, 28.1, 24.8, 17.9; HRMS (ES) Calculated for $\mathrm{C}_{27} \mathrm{H}_{27} \mathrm{~F}_{6} \mathrm{O}_{4}[\mathrm{M}+\mathrm{H}]^{+}$: 529.1808 found 529.1804. Calculated for $\mathrm{C}_{27} \mathrm{H}_{26} \mathrm{~F}_{6} \mathrm{O}_{4} \mathrm{Na}[\mathrm{M}+\mathrm{Na}]^{+}:$551.1627, found 551.1623.

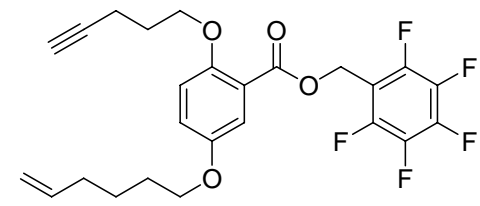

20A 
${ }^{1} \mathbf{H}$ NMR $\left(\mathrm{CDCl}_{3}, 400 \mathrm{MHz}\right) \delta 7.32(\mathrm{~d}, J=3.2 \mathrm{~Hz}, 1 \mathrm{H}), 7.00(\mathrm{dd}, J=9.0,3.2 \mathrm{~Hz}, 1 \mathrm{H}), 6.89(\mathrm{~d}, J=$ $9.1 \mathrm{~Hz}, 1 \mathrm{H}), 5.83(\mathrm{~m}, 1 \mathrm{H}), 5.40(\mathrm{~s}, 2 \mathrm{H}), 5.05-4.98(\mathrm{~m}, 2 \mathrm{H}), 4.04(\mathrm{t}, J=6.0 \mathrm{~Hz}, 2 \mathrm{H}), 3.92(\mathrm{t}, J=6.4$ $\mathrm{Hz}, 2 \mathrm{H}), 2.33-2.30(\mathrm{~m}, 2 \mathrm{H}), 2.13-2.10(\mathrm{~m}, 2 \mathrm{H}), 1.95-1.91(\mathrm{~m}, 3 \mathrm{H}), 1.79-1.75(\mathrm{~m}, 2 \mathrm{H}) ; 1.59-1.53$ $(\mathrm{m}, 2 \mathrm{H}) ;{ }^{13} \mathrm{C}$ NMR $\left(\mathrm{CDCl}_{3}, 100 \mathrm{MHz}\right) \delta 165.2,152.5,152.2,138.1,120.4,119.1,116.6,114.6$, 114.4, 83.0, 68.4, 68.1, 67.4, 53.3, 33.0, 28.3, 27.9, 24.9, 14.7; HRMS (ES) Calculated for $\mathrm{C}_{25} \mathrm{H}_{24} \mathrm{~F}_{5} \mathrm{O}_{4}[\mathrm{M}+\mathrm{H}]^{+}: 483.1589$ found 483.1572. Calculated for $\mathrm{C}_{25} \mathrm{H}_{23} \mathrm{~F}_{5} \mathrm{O}_{4} \mathrm{Na}[\mathrm{M}+\mathrm{Na}]^{+}$: 505.1409, found 505.1449.

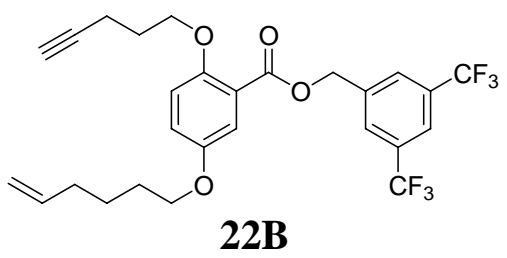

${ }^{1} \mathbf{H}$ NMR $\left(\mathrm{CDCl}_{3}, 400 \mathrm{MHz}\right) \delta 7.91(\mathrm{~s}, 2 \mathrm{H}), 7.85(\mathrm{~s}, 1 \mathrm{H}), 7.36(\mathrm{~d}, J=3.1 \mathrm{~Hz}, 1 \mathrm{H}), 7.04(\mathrm{dd}, J=9.0$, $3.1 \mathrm{~Hz}, 1 \mathrm{H}), 6.93(\mathrm{~d}, J=9.0 \mathrm{~Hz}, 1 \mathrm{H}), 5.80(\mathrm{~m}, 1 \mathrm{H}), 5.43(\mathrm{~s}, 2 \mathrm{H}), 5.04-4.96(\mathrm{~m}, 2 \mathrm{H}), 4.07(\mathrm{t}, J=6.0$ $\mathrm{Hz}, 2 \mathrm{H}), 3.94(\mathrm{t}, J=6.5 \mathrm{~Hz}, 2 \mathrm{H}), 2.34-2.30(\mathrm{~m}, 2 \mathrm{H}), 2.15-2.10(\mathrm{~m}, 2 \mathrm{H}), 1.96-1.76(\mathrm{~m}, 3 \mathrm{H}), 1.60-$ $1.53(\mathrm{~m}, 2 \mathrm{H}), 1.71-1.50(\mathrm{~m}, 2 \mathrm{H}) ;{ }^{13} \mathbf{C} \mathbf{~ N M R}\left(\mathrm{CDCl}_{3}, 100 \mathrm{MHz}\right) \delta 165.8,152.8,152.6,138.7,138.4$, $131.7\left(\mathrm{q}, \mathrm{CF}_{3}\right), 128.1,127.2,124.5,122.2,122.1,122.0,121.8,120.8,119.8,115.2,114.8,83.2$, 68.8, 68.5, 67.8, 64.9, 33.4, 28.6, 28.0, 25.2, 14.9; HRMS (ES) Calculated for $\mathrm{C}_{27} \mathrm{H}_{27} \mathrm{~F}_{6} \mathrm{O}_{4}[\mathrm{M}+\mathrm{H}]^{+}$: 529.1808 found 529.1796. Calculated for $\mathrm{C}_{27} \mathrm{H}_{26} \mathrm{~F}_{6} \mathrm{O}_{4} \mathrm{Na}[\mathrm{M}+\mathrm{Na}]^{+}$: 551.1627, found 551.1607.

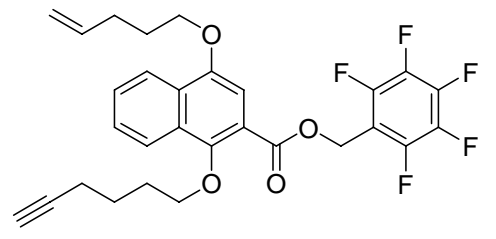

24A

${ }^{1} \mathbf{H}$ NMR $\left(\mathrm{CDCl}_{3}, 400 \mathrm{MHz}\right) \delta 8.27(\mathrm{~m}, 1 \mathrm{H}), 8.15(\mathrm{~m}, 1 \mathrm{H}), 7.59(\mathrm{~m}, 2 \mathrm{H}), 7.11(\mathrm{~s}, 1 \mathrm{H}), 5.90(\mathrm{~m}, 1 \mathrm{H})$, $5.50(\mathrm{~s}, 2 \mathrm{H}), 5.12-5.01(\mathrm{~m}, 2 \mathrm{H}), 4.15(\mathrm{t}, J=6.3 \mathrm{~Hz}, 2 \mathrm{H}), 4.02(\mathrm{t}, J=6.5 \mathrm{~Hz}, 2 \mathrm{H}), 2.35-2.30(\mathrm{~m}$, $4 \mathrm{H})$, 2.05- $1.93(\mathrm{~m}, 5 \mathrm{H}), 1.82-1.75(\mathrm{~m}, 2 \mathrm{H}) ;{ }^{13} \mathbf{C} \mathbf{~ N M R}\left(\mathrm{CDCl}_{3}, 100 \mathrm{MHz}\right) \delta$ 165.7, 151.3, 150.7, 137.7, 129.3, 129.2, 128.0, 127.1, 123.5, 122.8, 117.8, 115.3, 104.1, 83.9, 75.7, 68.6, 67.5, 53.9, 30.3, 29.3, 28.4, 25.0, 18.3; HRMS (ES) Calculated for $\mathrm{C}_{29} \mathrm{H}_{26} \mathrm{~F}_{5} \mathrm{O}_{4}[\mathrm{M}+\mathrm{H}]^{+}: 533.1745$ found 533.1768. Calculated for $\mathrm{C}_{29} \mathrm{H}_{25} \mathrm{~F}_{5} \mathrm{O}_{4} \mathrm{Na}[\mathrm{M}+\mathrm{Na}]^{+}:$555.1571, found 555.1594.

Ene-Yne precursor 26B was prepared following this sequence: 


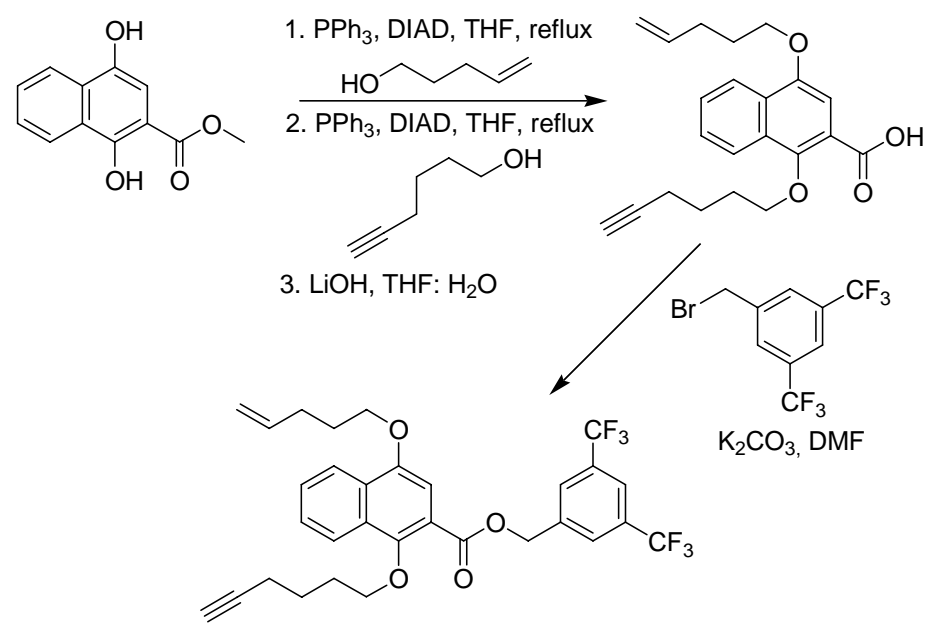

Figure SI4: Preparation of metathesis precursor 26B

In a round bottom flask, methyl-1, 4-dihydroxy-2-naphthanoic acid (500mg, $2.29 \mathrm{mmol}, 1.0$ eq.) was added to triphenylphosphine $(720 \mathrm{mg}, 2.75 \mathrm{mmol}, 1.2$ eq.) and pent-4-en-1-ol $(0.115 \mathrm{~mL}, 1.15$ mmol, 0.5 eq. $)^{4}$ was added. This mixture was then dissolved in dry THF $(12 \mathrm{~mL})$ and heated to reflux. DIAD $(0.56 \mathrm{~mL}, 2.75 \mathrm{mmol}, 1.2 \mathrm{eq}$.) was then added drop wise and the reaction was left under reflux and monitored by TLC. Typically after 10-15 hours the reaction was complete and no starting material was observed by TLC. The reaction mixture was then concentrated, dry-packed and purified by flash column silica chromatography using (Hexanes: Ethyl acetate 20:1). The product was immediately carried onto the second alkylation. In a round bottom flask, the mono-alkylated product (320 mg, $1.12 \mathrm{mmol}, 1.2 \mathrm{eq}$.) was added to triphenylphosphine (470 mg, $1.8 \mathrm{mmol}, 1.6 \mathrm{eq}$.) and hex-5-yn-1-ol $(0.145 \mathrm{~mL}, 1.34 \mathrm{mmol}, 1.2$ eq.) was added. This mixture was then dissolved in dry THF (10 mL) and heated to reflux. While under reflux, DIAD (0.265 mL, $1.8 \mathrm{mmol}, 1.6 \mathrm{eq}$.) was added drop wise and the reaction was left under reflux and monitored by TLC. After 15 hours, the reaction mixture was concentrated, dry-packed and purified by flash chromatography using (Hexanes: Ethyl acetate 10:1). The product was subsequently dissolved in a mixture of THF: H2O (4:1, $30 \mathrm{~mL})$ and $\mathrm{LiOH}(230 \mathrm{mg}, 9.5 \mathrm{mmol}, 10$ eq.) was added. The mixture was stirred at reflux for 18 hours. The reaction mixture was then quenched with $\mathrm{HCl}(10 \%)$ until acidic $\mathrm{pH}$ was obtained. The mixture was extracted many times with diethyl ether and washed with brine $(2 * 30 \mathrm{~mL})$. The organic phase was dried over $\mathrm{MgSO}_{4}$ and evaporated to afford a crude solid. The crude solid (316 $\mathrm{mg}, 0.90 \mathrm{mmol}, 1.0$ equiv.) is dissolved at room temperature in dry DMF. $\mathrm{K}_{2} \mathrm{CO}_{3}(740 \mathrm{mg}, 5.5$ mmol, 5.0 equiv.) is added. Then 3, 5-di (trifluoromethyl)benzyl bromide (330 mg, $1.1 \mathrm{mmol}, 1.2$ equiv.) is added to the mixture. The reaction was monitored by TLC (Hexanes: Ethyl acetate $7: 1$ ) and was typically complete after 1 hour, although reaction could be left overnight at room temperature. The DMF is then evaporated under vacuum, the reaction mixture acidified with $\mathrm{HCl}$ (10\%) until no more $\mathrm{CO}_{2}$ was formed. The mixture was extracted many times with diethyl ether and washed with brine $\left(2 * 30 \mathrm{~mL}\right.$ ). After drying over $\mathrm{MgSO}_{4}$ and evaporation the residue was drypacked and purified by flash column silica chromatography using (Hexanes: Ethyl acetate 10:1) to afford the product as a colourless oil.

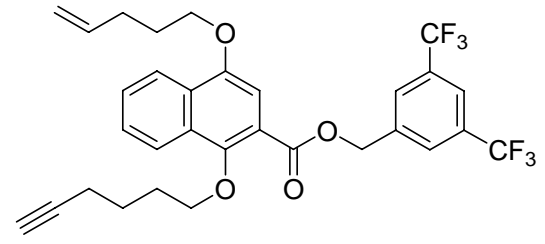

\footnotetext{
${ }^{4}$ This stochiometry is important to avoid the formation of dialkylated product.
} 
${ }^{1} \mathbf{H}$ NMR $\left(\mathrm{CDCl}_{3}, 400 \mathrm{MHz}\right) \delta 8.33(\mathrm{~m}, 1 \mathrm{H}), 8.21(\mathrm{~m}, 1 \mathrm{H}), 8.00(\mathrm{~s}, 2 \mathrm{H}), 7.91(\mathrm{~s}, 1 \mathrm{H}), 7.63-7.59(\mathrm{~m}$, 2H), $7.19(\mathrm{~s}, 1 \mathrm{H}), 5.90(\mathrm{~m}, 1 \mathrm{H}), 5.53(\mathrm{~s}, 2 \mathrm{H}), 5.14-5.05(\mathrm{~m}, 2 \mathrm{H}), 4.17(\mathrm{t}, J=6.2 \mathrm{~Hz}, 2 \mathrm{H}), 4.05(\mathrm{t}, J=$ $6.5 \mathrm{~Hz}, 2 \mathrm{H}), 2.37-2.27(\mathrm{~m}, 4 \mathrm{H}), 2.06-1.99(\mathrm{~m}, 5 \mathrm{H}), 1.82-1.70(\mathrm{~m}, 2 \mathrm{H}) ;{ }^{13} \mathbf{C} \mathbf{~ N M R}\left(\mathrm{CDCl}_{3}, 100\right.$ $\mathrm{MHz}) \delta 165.7,151.3,150.7,138.7,137.6,131.4\left(\mathrm{q}, \mathrm{CF}_{3}\right), 129.3,129.1,128.1,127.9,127.1,124.5$, 123.4, 122.3, 122.1, 117.9, 115.2, 103.9, 83.9, 75.6, 68.6, 67.4, 65.0, 31.5, 30.2, 29.3, 25.2, 18.2; HRMS (ES) Calculated for $\mathrm{C}_{31} \mathrm{H}_{29} \mathrm{~F}_{6} \mathrm{O}_{4}[\mathrm{M}+\mathrm{H}]^{+}:$579.1965, found 579.1963. Calculated for $\mathrm{C}_{31} \mathrm{H}_{28} \mathrm{~F}_{6} \mathrm{O}_{4} \mathrm{Na}[\mathrm{M}+\mathrm{Na}]^{+}: 601.1784$, found 601.1785 .

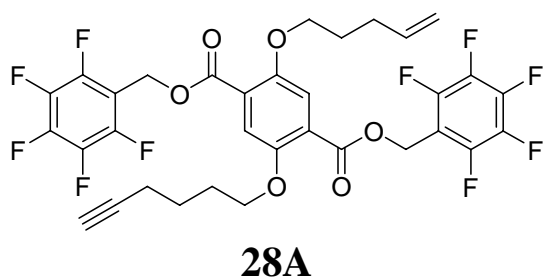

${ }^{1} \mathbf{H}$ NMR $\left(\mathrm{CDCl}_{3}, 400 \mathrm{MHz}\right) \delta 7.33(\mathrm{~s}, 2 \mathrm{H}), 5.77(\mathrm{~m}, 1 \mathrm{H}), 5.42(\mathrm{~s}, 4 \mathrm{H}), 5.01-4.97(\mathrm{~m}, 2 \mathrm{H}), 4.00-$ $3.94(\mathrm{~m}, 4 \mathrm{H}), 2.24-2.20(\mathrm{~m}, 2 \mathrm{H}), 2.14-2.12(\mathrm{~m}, 2 \mathrm{H}), 1.95(\mathrm{~s}, 1 \mathrm{H}), 1.86-1.64(\mathrm{~m}, 4 \mathrm{H}), 1.65-1.61$ $(\mathrm{m}, 2 \mathrm{H}) ;{ }^{13} \mathbf{C} \mathbf{N M R}\left(\mathrm{CDCl}_{3}, 100 \mathrm{MHz}\right) \delta 165.2,165.1,151.8,151.7,137.4,123.5,116.6,116.5$, 115.2, 83.8, 68.9, 68.7, 68.6, 54.0, 29.8, 28.1, 28.0, 24.8, 18.0; HRMS (ES) Calculated for $\mathrm{C}_{33} \mathrm{H}_{25} \mathrm{~F}_{10} \mathrm{O}_{6}[\mathrm{M}+\mathrm{H}]^{+}: 707.1486$ found 707.1483. Calculated for $\mathrm{C}_{33} \mathrm{H}_{24} \mathrm{~F}_{10} \mathrm{O}_{6} \mathrm{Na}[\mathrm{M}+\mathrm{Na}]^{+}$: 729.1311 , found 729.1316 .

Ene-Yne precursor 30B was prepared following this sequence:

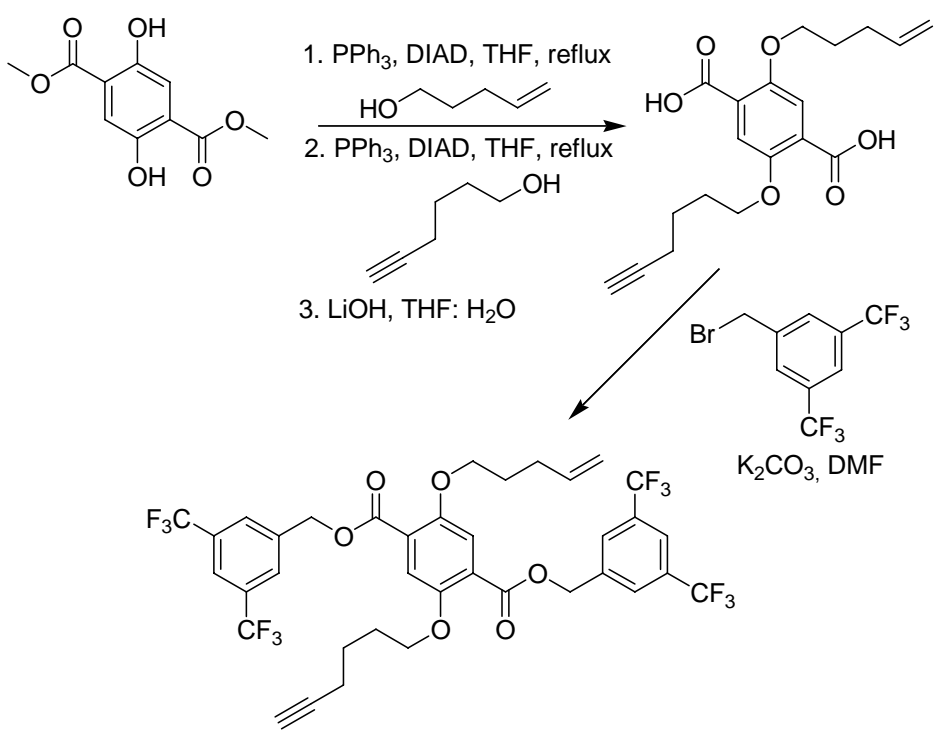

Figure SI5: Preparation of metathesis precursor 30B

In a round bottom flask, methyl-2, 5-dihydroxybenzene dicarboxylate (250 $\mathrm{mg}, 1.1 \mathrm{mmol}, 1.0$ eq.) was added to triphenylphosphine (434 mg, $1.66 \mathrm{mmol}, 1.5$ eq.) and pent-4-en-1-ol (0.056 mL, 0.55 mmol, 0.50 eq. $)^{5}$ was added. This mixture was then dissolved in dry THF $(6 \mathrm{~mL})$ and heated to

\footnotetext{
${ }^{5}$ This stochiometry is important to avoid the formation of dialkylated product.
} 
reflux. DIAD (0.338 mL, $1.66 \mathrm{mmol}, 1.5$ eq.) was then added drop wise and the reaction was left under reflux and monitored by TLC. Typically after 10-15 hours the reaction was complete and no starting material was observed by TLC. The reaction mixture was then concentrated, dry-packed and purified by flash column silica chromatography using (Hexanes: Ethyl acetate 20:1). The product was immediately carried onto the second alkylation. In a round bottom flask, the mono-alkylated product (177 mg, .605 mmol, 1.0 eq.) was added to triphenylphosphine (253 mg, $0.97 \mathrm{mmol}, 1.6 \mathrm{eq}$.) and hex-5-yn-1-ol (0.082 mL, $0.726 \mathrm{mmol}, 1.2 \mathrm{eq}$.) was added. This mixture was then dissolved in dry THF $(6 \mathrm{~mL})$ and heated to reflux. While under reflux, DIAD $(0.20 \mathrm{~mL}, 0.97 \mathrm{mmol}, 1.6$ eq. $)$ was added drop wise and the reaction was left under reflux and monitored by TLC. After 15 hours, the reaction mixture was concentrated, dry-packed and purified by flash chromatography using (Hexanes: Ethyl acetate 10:1). The product was subsequently dissolved in a mixture of THF: $\mathrm{H}_{2} \mathrm{O}$ (4:1, $20 \mathrm{~mL})$ and $\mathrm{LiOH}(120 \mathrm{mg}, 5 \mathrm{mmol}, 10 \mathrm{eq}$.) was added. The mixture was stirred at reflux for 15 hours. The reaction mixture was then quenched with $\mathrm{HCl}(10 \%)$ until acidic $\mathrm{pH}$ was obtained. The mixture was extracted many times with diethyl ether and washed with brine $(2 * 25 \mathrm{~mL})$. The organic phase was separated, dried $\left(\mathrm{MgSO}_{4}\right)$ and evaporated to afford a crude solid. The crude solid (160 mg, $0.46 \mathrm{mmol}, 1.0$ equiv.) is dissolved at room temperature in dry DMF and 3, 5di(trifluoromethyl)benzyl bromide (169 mg, $0.55 \mathrm{mmol}, 1.2 \mathrm{eq}$.) is added to the mixture. $\mathrm{K}_{2} \mathrm{CO}_{3}$ (370 mg, $2.75 \mathrm{mmol}, 5.0$ equiv.) is then added. The reaction was monitored by TLC (Hexanes: Ethyl acetate $7: 1$ ) and was typically complete after 1 hour, although reactions could be left overnight at room temperature. The DMF is then evaporated under vacuum, the reaction mixture acidified with $\mathrm{HCl}(10 \%)$ until no more $\mathrm{CO}_{2}$ was formed. The mixture was extracted many times with diethyl ether and washed with brine $\left(2 * 25 \mathrm{~mL}\right.$ ). After drying over $\mathrm{MgSO}_{4}$ and evaporation the residue was drypacked and purified by flash column silica chromatography using (Hexanes: Ethyl acetate 10:1) to afford the product as a colourless oil.

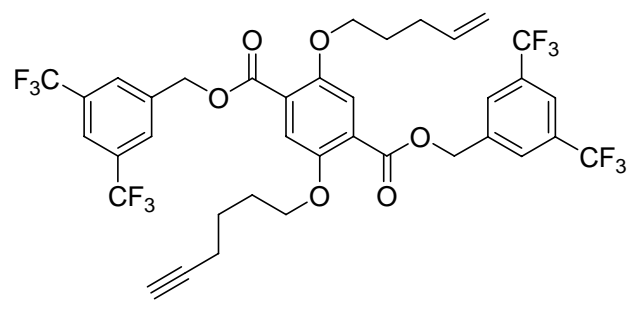

30B

${ }^{1} \mathbf{H}$ NMR $\left(\mathrm{CDCl}_{3}, 400 \mathrm{MHz}\right) \delta 7.92(\mathrm{~s}, 2 \mathrm{H}), 7.87(\mathrm{~s}, 1 \mathrm{H}), 7.43(\mathrm{~s}, 2 \mathrm{H}), 5.75(\mathrm{~m}, 1 \mathrm{H}), 5.47(\mathrm{~s}, 4 \mathrm{H})$, 4.97- $4.92(\mathrm{~m}, 2 \mathrm{H}), 4.07-4.01(\mathrm{~m}, 4 \mathrm{H}), 2.19-2.12(\mathrm{~m}, 4 \mathrm{H}), 1.91-1.80(\mathrm{~m}, 5 \mathrm{H}), 1.70-1.55(\mathrm{~m}, 2 \mathrm{H})$; ${ }^{13} \mathbf{C}$ NMR $\left(\mathrm{CDCl}_{3}, 100 \mathrm{MHz}\right) \delta 165.5,165.4,151.8,151.7,138.4,138.3,137.2,131.8\left(\mathrm{q}, \mathrm{CF}_{3}\right)$, 128.0, 127.2, 124.5, 123.8, 122.3, 122.2, 121.7, 119.1, 116.6, 116.5, 115.3, 83.8, 68.9, 68.7, 68.6, 65.3, 65.2, 29.7, 28.1, 28.0, 24.8, 17.9; HRMS (ES) Calculated for $\mathrm{C}_{37} \mathrm{H}_{31} \mathrm{~F}_{12} \mathrm{O}_{6}[\mathrm{M}+\mathrm{H}]^{+}$: 799.1924, found 799.1925. Calculated for $\mathrm{C}_{37} \mathrm{H}_{30} \mathrm{~F}_{12} \mathrm{O}_{6} \mathrm{Na}[\mathrm{M}+\mathrm{Na}]^{+}:$821.1743, found 821.1743.

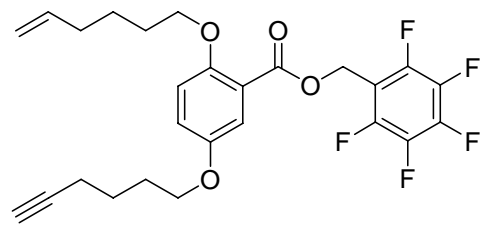

32A

${ }^{1} \mathbf{H}$ NMR $\left(\mathrm{CDCl}_{3}, 400 \mathrm{MHz}\right) \delta 7.28(\mathrm{~d}, J=3.1 \mathrm{~Hz}, 1 \mathrm{H}), 7.00(\mathrm{dd}, J=9.5,3.1 \mathrm{~Hz}, 1 \mathrm{H}), 6.86(\mathrm{~d}, J=$ $9.7 \mathrm{~Hz}, 1 \mathrm{H}), 5.78(\mathrm{~m}, 1 \mathrm{H}), 5.39(\mathrm{~s}, 2 \mathrm{H}), 5.03-4.94(\mathrm{~m}, 2 \mathrm{H}), 3.95-3.92(\mathrm{t}, J=6.1 \mathrm{~Hz}, 4 \mathrm{H}), 2.27-2.24$ $(\mathrm{m}, 2 \mathrm{H}), 2.09-2.06(\mathrm{~m}, 2 \mathrm{H}), 1.95(\mathrm{t}, J=2.5 \mathrm{~Hz}, 1 \mathrm{H}), 1.90-1.85(\mathrm{~m}, 2 \mathrm{H}), 1.76-1.67(\mathrm{~m}, 4 \mathrm{H}), 1.52-$ 
$1.49(\mathrm{~m}, 2 \mathrm{H}) ;{ }^{13} \mathbf{C} \mathbf{N M R}\left(\mathrm{CDCl}_{3}, 100 \mathrm{MHz}\right) \delta 165.5,153.1,152.3,138.4,120.6,119.6,116.8,114.9$, 114.6, 84.0, 69.4, 68.6, 67.9, 53.6, 33.3, 28.6, 28.2, 25.1, 24.9, 18.1; HRMS (ES) Calculated for $\mathrm{C}_{26} \mathrm{H}_{26} \mathrm{~F}_{5} \mathrm{O}_{4}[\mathrm{M}+\mathrm{H}]^{+}: 497.1745$ found 497.1739. Calculated for $\mathrm{C}_{26} \mathrm{H}_{25} \mathrm{~F}_{5} \mathrm{O}_{4} \mathrm{Na}[\mathrm{M}+\mathrm{Na}]^{+}$: 519.1571 , found 519.1568 .

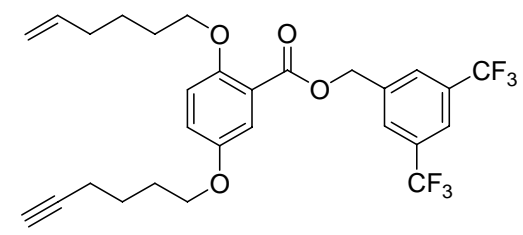

$34 B$

${ }^{1} \mathbf{H}$ NMR $\left(\mathrm{CDCl}_{3}, 400 \mathrm{MHz}\right) \delta 7.92(\mathrm{~s}, 2 \mathrm{H}), 7.85(\mathrm{~s}, 1 \mathrm{H}), 7.36(\mathrm{~d}, J=3.0 \mathrm{~Hz}, 1 \mathrm{H}), 7.03(\mathrm{dd}, J=9.1$, $3.0 \mathrm{~Hz}, 1 \mathrm{H}), 6.91(\mathrm{~d}, J=9.1 \mathrm{~Hz}, 1 \mathrm{H}), 5.73(\mathrm{~m}, 1 \mathrm{H}), 5.44(\mathrm{~s}, 2 \mathrm{H}), 4.99-4.91(\mathrm{~m}, 2 \mathrm{H}), 4.00-3.94(\mathrm{~m}$, 4H), 2.28- $2.21(\mathrm{~m}, 2 \mathrm{H}), 2.10-2.00(\mathrm{~m}, 2 \mathrm{H}), 1.97(\mathrm{t}, J=2.6 \mathrm{~Hz}, 1 \mathrm{H}), 1.87-1.80(\mathrm{~m}, 2 \mathrm{H}), 1.77-1.65$ $(\mathrm{m}, 4 \mathrm{H}), 1.55-1.45(\mathrm{~m} \mathrm{2H}) ;{ }^{13} \mathbf{C}$ NMR $\left(\mathrm{CDCl}_{3}, 100 \mathrm{MHz}\right) \delta 166.0,153.1,152.3,138.8,138.4,131.8$ (q, $\left.\mathrm{CF}_{3}\right), 128.0,127.2,124.5,122.0,121.8,120.8,119.7,116.7,114.9,114.6,84.0,69.4,68.6,68.0$, 64.9, 33.2, 28.5, 25.1, 25.0, 24.8, 18.1; HRMS (ES) Calculated for $\mathrm{C}_{28} \mathrm{H}_{29} \mathrm{~F}_{6} \mathrm{O}_{4}[\mathrm{M}+\mathrm{H}]^{+}: 543.1965$, found 543.1972. Calculated for $\mathrm{C}_{28} \mathrm{H}_{28} \mathrm{~F}_{6} \mathrm{O}_{4} \mathrm{Na}[\mathrm{M}+\mathrm{Na}]^{+}: 565.1784$, found 565.1790.

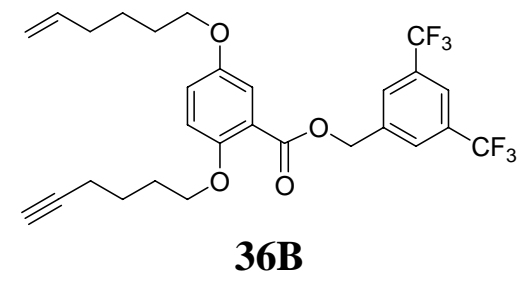

${ }^{1} \mathbf{H}$ NMR $\left(\mathrm{CDCl}_{3}, 400 \mathrm{MHz}\right) \delta 7.95(\mathrm{~s}, 2 \mathrm{H}), 7.88(\mathrm{~s}, 1 \mathrm{H}), 7.38(\mathrm{~d}, J=3.0 \mathrm{~Hz}, 1 \mathrm{H}), 7.06(\mathrm{dd}, J=9.1$, $3.0 \mathrm{~Hz}, 1 \mathrm{H}), 6.94(\mathrm{~d}, J=9.1 \mathrm{~Hz}, 1 \mathrm{H}), 5.83(\mathrm{~m}, 1 \mathrm{H}), 5.47(\mathrm{~s}, 2 \mathrm{H}), 5.07-4.97(\mathrm{~m}, 2 \mathrm{H}), 4.04(\mathrm{t}, J=6.0$ $\mathrm{Hz}, 2 \mathrm{H}), 3.96(\mathrm{t}, J=6.4 \mathrm{~Hz}, 2 \mathrm{H}), 2.22-2.10(\mathrm{~m}, 4 \mathrm{H}), 1.94-1.78(\mathrm{~m}, 5 \mathrm{H}), 1.69-1.50(\mathrm{~m}, 4 \mathrm{H}) ;{ }^{13} \mathrm{C}$ NMR $\left(\mathrm{CDCl}_{3}, 100 \mathrm{MHz}\right) \delta 165.8,152.9,152.5,138.8,138.4,131.7\left(\mathrm{q}, \mathrm{CF}_{3}\right), 128.0,127.2,124.5$, 122.0, 121.8, 120.8, 119.7, 116.7, 114.9, 114.7, 83.9, 68.9, 68.5, 68.4, 64.8, 33.3, 28.6, 28.1, 25.2, 24.8, 17.9; HRMS (ES) Calculated for $\mathrm{C}_{28} \mathrm{H}_{29} \mathrm{~F}_{6} \mathrm{O}_{4}[\mathrm{M}+\mathrm{H}]^{+}:$543.1965, found 543.1969. Calculated for $\mathrm{C}_{28} \mathrm{H}_{28} \mathrm{~F}_{6} \mathrm{O}_{4} \mathrm{Na}[\mathrm{M}+\mathrm{Na}]^{+}: 565.1784$, found 565.1781 
Ene-Yne precursor 38B was prepared following this sequence:

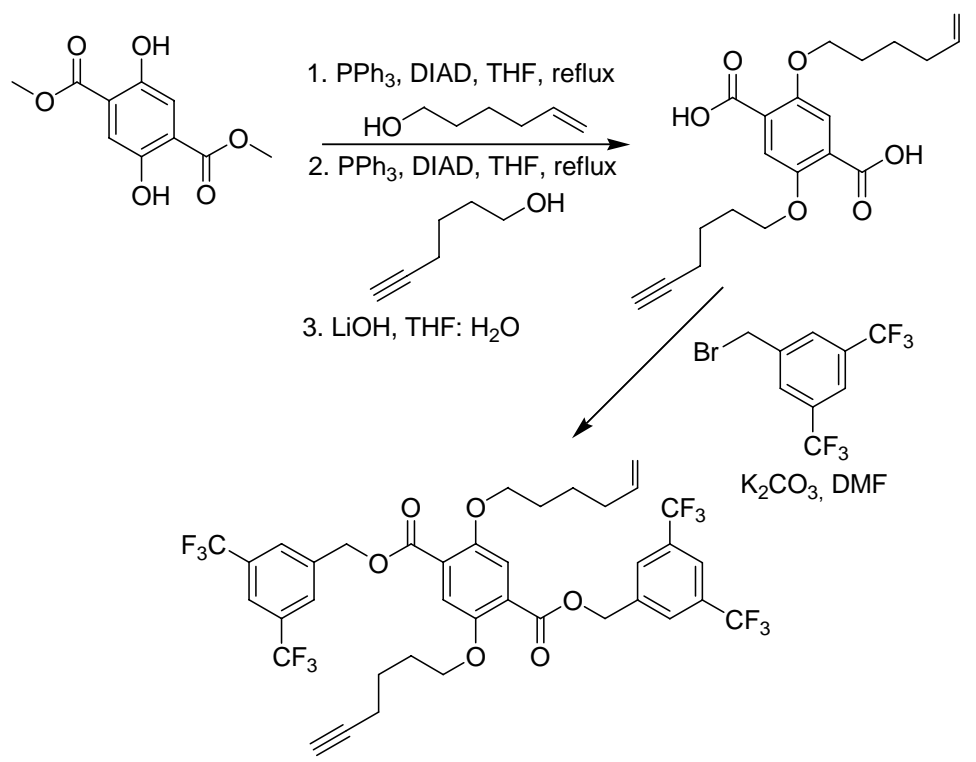

Figure SI6: Preparation of metathesis precursor 38B

In a round bottom flask, methyl-2, 5-dihydroxybenzene dicarboxylate (250 $\mathrm{mg}, 1.1 \mathrm{mmol}, 1.0$ eq.) was added to triphenylphosphine (434 mg, $1.66 \mathrm{mmol}, 1.5$ eq.) and Hexen-5-en-1-ol (0.066 mL, 0.55 mmol, 0.50 eq. $\left.{ }^{6}\right)$ was added. This mixture was then dissolved in dry THF $(10 \mathrm{~mL})$ and heated to reflux. DIAD (0.338 $\mathrm{mL}, 1.66 \mathrm{mmol}, 1.5 \mathrm{eq}$.$) was then added drop wise and the reaction was left$ under reflux and monitored by TLC. Typically after 10-15 hours the reaction was complete and no starting material was observed by TLC. The reaction mixture was then concentrated, dry-packed and purified by flash column silica chromatography using (Hexanes: Ethyl acetate 20:1). The product was immediately carried onto the second alkylation. In a round bottom flask, the mono-alkylated product (185 mg, $.60 \mathrm{mmol}, 1.0$ eq.) was added to triphenylphosphine ( $253 \mathrm{mg}, 0.97 \mathrm{mmol}, 1.6 \mathrm{eq}$.) and hex-5-yn-1-ol $(0.080 \mathrm{~mL}, 0.72 \mathrm{mmol}, 1.2$ eq.) was added. This mixture was then dissolved in dry THF $(6 \mathrm{~mL})$ and heated to reflux. While under reflux, DIAD $(0.19 \mathrm{~mL}, 0.95 \mathrm{mmol}, 1.6 \mathrm{eq}$. $)$ was added drop wise and the reaction was left under reflux and monitored by TLC. After 15 hours, the reaction mixture was concentrated, dry-packed and purified by flash chromatography using (Hexanes: Ethyl acetate 10:1). The product was subsequently dissolved in a mixture of THF: $\mathrm{H}_{2} \mathrm{O}$ $(4: 1,20 \mathrm{~mL})$ and $\mathrm{LiOH}(120 \mathrm{mg}, 5 \mathrm{mmol}, 10 \mathrm{eq}$.) was added. The mixture was stirred at reflux for 15 hours. The reaction mixture was then quenched with $\mathrm{HCl}(10 \%)$ until acidic $\mathrm{pH}$ was obtained. The mixture was extracted many times with diethyl ether and washed with brine $(2 * 25 \mathrm{~mL})$. The organic phase was separated, dried $\left(\mathrm{MgSO}_{4}\right)$ and evaporated to afford a crude solid. The crude solid (160 mg, $0.46 \mathrm{mmol}, 1.0$ equiv.) is dissolved at room temperature in dry DMF and 3, 5-di (trifluoromethyl)benzyl bromide ( $170 \mathrm{mg}, 0.55 \mathrm{mmol}, 1.2 \mathrm{eq}$.) is added to the mixture. $\mathrm{K}_{2} \mathrm{CO}_{3}(370$ $\mathrm{mg}, 2.75 \mathrm{mmol}, 5.0$ equiv.) is then added. The reaction was monitored by TLC (Hexanes: Ethyl acetate 7:1) and was typically complete after 1 hour, although reactions could be left overnight at room temperature. The DMF is then evaporated under vacuum, the reaction mixture acidified with $\mathrm{HCl}(10 \%)$ until no more $\mathrm{CO}_{2}$ was formed. The mixture was extracted many times with diethyl ether and washed with brine $\left(2 * 25 \mathrm{~mL}\right.$ ). After drying over $\mathrm{MgSO}_{4}$ and evaporation the residue was drypacked and purified by flash column silica chromatography using (Hexanes: Ethyl acetate 10:1) to afford the product as a colourless oil.

\footnotetext{
${ }^{6}$ This stochiometry is important to avoid the formation of dialkylated product.
} 


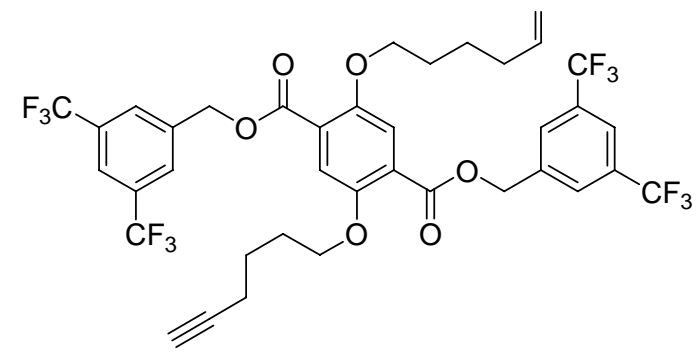

$38 B$

${ }^{1} \mathbf{H}$ NMR $\left(\mathrm{CDCl}_{3}, 400 \mathrm{MHz}\right) \delta 7.92(\mathrm{~s}, 4 \mathrm{H}), 7.87(\mathrm{~s}, 2 \mathrm{H}), 7.43(\mathrm{~s}, 2 \mathrm{H}), 5.71(\mathrm{~m}, 1 \mathrm{H}), 5.47(\mathrm{~s}, 4 \mathrm{H})$, 4.98- $4.92(\mathrm{~m}, 2 \mathrm{H}), 4.07-4.01(\mathrm{~m}, 4 \mathrm{H}), 2.19-2.16(\mathrm{~m}, 2 \mathrm{H}), 2.10-1.97(\mathrm{~m}, 2 \mathrm{H}), 1.90-1.87(\mathrm{~m}, 3 \mathrm{H})$, $1.80-1.70(\mathrm{~m}, 2 \mathrm{H}), 1.68-1.60(\mathrm{~m}, 2 \mathrm{H}), 1.57-1.45(\mathrm{~m}, 2 \mathrm{H}) ;{ }^{13} \mathbf{C} \mathbf{N M R}\left(\mathrm{CDCl}_{3}, 100 \mathrm{MHz}\right) \delta 165.5$, $165.4,151.9,151.8,138.5,138.2,131.8\left(\mathrm{q}, \mathrm{CF}_{3}\right), 128.0,127.2,124.5,123.9,123.8,122.2,121.8$, 119.1, 116.6, 116.5, 114.7, 83.8, 69.5, 69.0, 68.6, 65.3, 33.1, 28.4, 28.0, 25.0, 24.8, 17.9; HRMS (ES) Calculated for $\mathrm{C}_{38} \mathrm{H}_{33} \mathrm{~F}_{12} \mathrm{O}_{6}[\mathrm{M}+\mathrm{H}]^{+}$: 813.2080, found 813.2079. Calculated for $\mathrm{C}_{38} \mathrm{H}_{32} \mathrm{~F}_{12} \mathrm{O}_{6} \mathrm{Na}[\mathrm{M}+\mathrm{Na}]^{+}:$835.1900, found 835.1900.

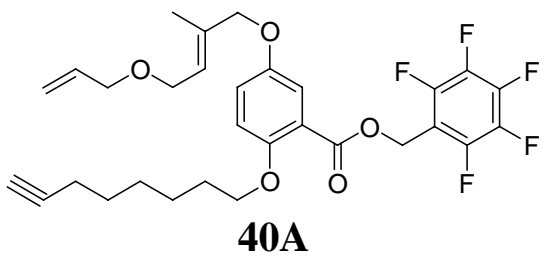

${ }^{1} \mathbf{H}$ NMR $\left(\mathrm{CDCl}_{3}, 400 \mathrm{MHz}\right) \delta 7.30(\mathrm{~d}, J=3.2 \mathrm{~Hz}, 1 \mathrm{H}), 7.10(\mathrm{dd}, J=9.1,3.2 \mathrm{~Hz}, 1 \mathrm{H}), 6.86(\mathrm{~d}, J=$ $9.1 \mathrm{~Hz}, 1 \mathrm{H}), 5.90(\mathrm{~m}, 1 \mathrm{H}), 5.72(\mathrm{td}, J=6.0,1.2 \mathrm{~Hz}, 1 \mathrm{H}), 5.39(\mathrm{~s}, 2 \mathrm{H}),, 5.29-5.17(\mathrm{~m}, 2 \mathrm{H}), 4.37(\mathrm{~s}$, $2 \mathrm{H}), 4.05(\mathrm{~d}, J=6.4 \mathrm{~Hz}, 2 \mathrm{H}), 3.97-3.92(\mathrm{~m}, 4 \mathrm{H}), 2.22-2.18(\mathrm{td}, J=9.6,2.6 \mathrm{~Hz}, 2 \mathrm{H}), 1.94(\mathrm{t}, J=2.6$ $\mathrm{Hz}, 1 \mathrm{H}), 1.77-1.71(\mathrm{~m}, 5 \mathrm{H}), 1.55-1.40(\mathrm{~m}, 6 \mathrm{H}) ;{ }^{13} \mathbf{C} \mathbf{~ N M R}\left(\mathrm{CDCl}_{3}, 100 \mathrm{MHz}\right) \delta 165.4,153.3$, 134.7, 134.6, 124.6, 120.9, 119.5, 117.4, 117.3, 117.2, 114.9, 84.5, 73.6, 71.2, 69.5, 68.2, 66.0, 29.0, 28.6, 28.3, 25.3, 18.3, 14.1. HRMS (ES) Calculated for $\mathrm{C}_{30} \mathrm{H}_{32} \mathrm{~F}_{5} \mathrm{O}_{5}[\mathrm{M}+\mathrm{H}]^{+}: 567.2172$ found 567.2167. Calculated for $\mathrm{C}_{30} \mathrm{H}_{31} \mathrm{~F}_{5} \mathrm{O}_{5} \mathrm{Na}[\mathrm{M}+\mathrm{Na}]^{+}: 589.1990$, found 589.1983 .

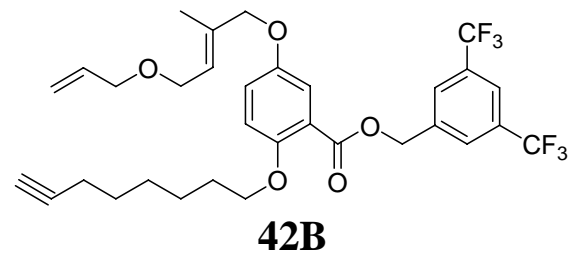

${ }^{1} \mathbf{H}$ NMR $\left(\mathrm{CDCl}_{3}, 400 \mathrm{MHz}\right) \delta 7.91(\mathrm{~s}, 2 \mathrm{H}), 7.85(\mathrm{~s}, 1 \mathrm{H}), 7.37(\mathrm{~d}, J=3.1 \mathrm{~Hz}, 1 \mathrm{H}), 7.04(\mathrm{dd}, J=$ 9.1,3.1 Hz, 1H), $6.90(\mathrm{~d}, J=9.1 \mathrm{~Hz}, 1 \mathrm{H}), 5.91(\mathrm{~m}, 1 \mathrm{H}), 5.73(\mathrm{td}, J=6.3,1.2 \mathrm{~Hz}, 1 \mathrm{H}), 5.43(\mathrm{~s}, 2 \mathrm{H}$,$) ,$ 5.28- $5.16(\mathrm{~m}, 2 \mathrm{H}), 4.39(\mathrm{~s}, 2 \mathrm{H}), 4.06(\mathrm{~d}, J=6.3 \mathrm{~Hz}, 2 \mathrm{H}), 4.00-3.95(\mathrm{~m}, 4 \mathrm{H}), 2.20-2.15(\mathrm{~m}, 2 \mathrm{H})$, $1.92(\mathrm{t}, J=2.6 \mathrm{~Hz}, 1 \mathrm{H}), 1.77-1.72(\mathrm{~m}, 5 \mathrm{H}), 1.53-1.35(\mathrm{~m}, 6 \mathrm{H}) ;{ }^{13} \mathbf{C} \mathbf{~ N M R}\left(\mathrm{CDCl}_{3}, 100 \mathrm{MHz}\right) \delta$ 165.9, 153.2, 152.0, 138.9, 134.7, $131.7\left(\mathrm{~m}, \mathrm{CF}_{3}\right), 127.9,127.2$, 124.5, 124.4, 122.0, 121.9, 121.8, 121.7, 121.0, 119.7, 117.3, 117.1, 114.9, 114.8, 84.4, 73.6, 71.2, 69.5, 68.2, 66.0, 53.6, 28.9, 28.6, 28.3, 25.3, 18.2, 14.0. HRMS (ES) Calculated for $\mathrm{C}_{32} \mathrm{H}_{35} \mathrm{~F}_{6} \mathrm{O}_{5}[\mathrm{M}+\mathrm{H}]^{+}: 613.2389$, found 613.2393. Calculated for $\mathrm{C}_{32} \mathrm{H}_{34} \mathrm{~F}_{6} \mathrm{O}_{5} \mathrm{Na}[\mathrm{M}+\mathrm{Na}]^{+}:$635.2208, found 635.2197. 


\section{General procedure for the direct ene-yne metathesis reaction}

A general procedure for en- yne metathesis reaction is detailed below. Following this description, the spectral data is indicated for each individual compound. Note that the determination of the configuration of the double bond $\left(E\right.$ or $Z$ ) was accomplished using ${ }^{1} \mathrm{H}$ NMR NOE and NOESY experiments. The $Z: E$ ratios were determined by ${ }^{1} \mathrm{H}$ NMR through analogy with en- yne products 21A and 27B (see the attached spectra section).

The $Z$ and $E$ isomers were quantified based on the relative integrations of the ${ }^{1} \mathrm{H}$ NMR signals for protons $\mathrm{H}_{\mathrm{a}}$ and $\mathrm{H}_{\mathrm{b}}$ (see Figure SI7). These assignments are consistent with those observed by Lee and co-workers. ${ }^{7}$ In all cases the two isomers produced are inseparable by flash column silica chromatography. Spectral data is reported for the major isomer in each case. The ${ }^{1} \mathrm{H}$ and ${ }^{13} \mathrm{C}$ NMR spectra of the Z:E mixtures are included in the Supporting Information as a reference. In addition, the majority of the macrocycles formed are atropisomeric. The diastereotopicity combined with the fact that the products are mixtures of $Z: E$ isomers renders some spectra quite complicated. As such, many products of en -yne metathesis were fully hydrogenated ${ }^{8}$ and the characterization data, ${ }^{1} \mathrm{H}$ and ${ }^{13} \mathrm{C}$ NMR spectra for a selection of these compounds are listed below.

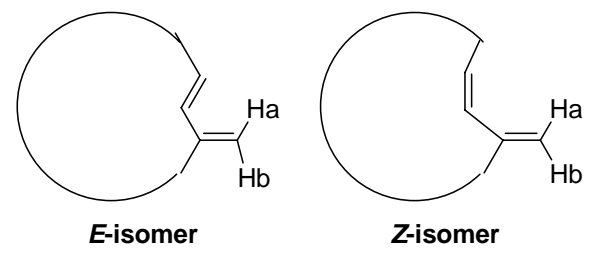

Figure SI7: Identification of $Z$ and $E$ isomers

A flame dried $500 \mathrm{~mL}$ tri-neck round bottom flask, equipped with a magnetic stirrer, reflux condenser, and isobar addition funnel or a syringe pump system is charged with catalyst G1, G2 or GH2 $(20 \mathrm{~mol} \%)$ and anhydrous $\mathrm{CH}_{2} \mathrm{Cl}_{2}$ or toluene (volume is determined by the amount needed to afford a final concentration of $[\mathrm{M}]=0.4 \times 10^{-4} \mathrm{M}$ after complete addition of the precursor). The catalyst solution is then placed at reflux $\left(\mathrm{CH}_{2} \mathrm{Cl}_{2}\right)$ or $110^{\circ} \mathrm{C}$ (toluene). The metathesis precursor in solution (approximately $50 \mathrm{~mL}$ ) is placed in the addition funnel or the syringe pumps system and added over $1 \mathrm{~h}$. After addition, the solution was allowed to stir at reflux for 1 additional hour to ensure complete conversion (reactions performed in $\mathrm{CH}_{2} \mathrm{Cl}_{2}$ all had to be left overnight). The reaction mixture was concentrated in vacuo, dry-packed and purified by flash column silica chromatography (Hexanes-Ethyl Acetate 20:1) to afford the desired 1, 3-diene paracyclophanes.

\footnotetext{
${ }^{7}$ Hansen, E. C.; Lee, D. J. Am. Chem. Soc. 2004; 126, 15074-15080 and references therein.

${ }^{8}$ Hydrogenation of 1, 3-diene paracyclophanes in solution in Ethyl Acetate: MeOH 10:1 using $\mathrm{H}_{2}(1 \mathrm{~atm})$ and $\mathrm{Pd}(\mathrm{OH})_{2} / \mathrm{C}(10 \mathrm{~mol} \%)$ as a catalyst.
} 


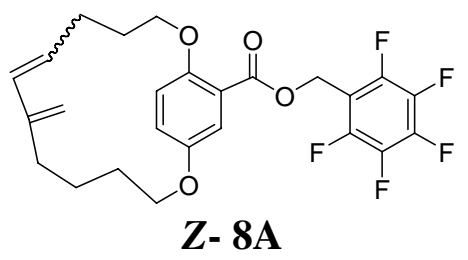

${ }^{1} \mathbf{H}$ NMR $\left(\mathrm{CDCl}_{3}, 400 \mathrm{MHz}\right) \delta 7.42(\mathrm{~d}, J=3.1 \mathrm{~Hz}, 1 \mathrm{H}), 7.10(\mathrm{dd}, J=9.0,3.0 \mathrm{~Hz}, 1 \mathrm{H}), 6.95(\mathrm{~d}, J=$ $9.0 \mathrm{~Hz}, 1 \mathrm{H}), 5.44-5.40(\mathrm{~m}, 2 \mathrm{H}), 5.18-5.14(\mathrm{~m}, 2 \mathrm{H}), 4.81(\mathrm{~d}, J=1.7 \mathrm{~Hz}, 1 \mathrm{H}), 4.82(\mathrm{~s}, 1 \mathrm{H}), 4.24-4.17$ $(\mathrm{m}, 4 \mathrm{H}), 2.08-2.04(\mathrm{~m}, 2 \mathrm{H}), 1.84-1.64(\mathrm{~m}, 4 \mathrm{H}), 1.39-1.08(\mathrm{~m}, 4 \mathrm{H}) ;{ }^{13} \mathbf{C} \mathbf{~ N M R}\left(\mathrm{CDCl}_{3}, 100 \mathrm{MHz}\right) \delta$ $164.9,155.7,152.7,145.8,131.5,128.5,124.6,121.7,121.6,120.4,111.0,72.2,70.1,53.7,34.6$, 30.7, 28.7, 27.0, 24.8; HRMS (ES) Calculated for $\mathrm{C}_{25} \mathrm{H}_{24} \mathrm{~F}_{5} \mathrm{O}_{4}[\mathrm{M}+\mathrm{H}]^{+}: 483.1589$ found 483.1601. Calculated for $\mathrm{C}_{25} \mathrm{H}_{23} \mathrm{~F}_{5} \mathrm{O}_{4} \mathrm{Na}[\mathrm{M}+\mathrm{Na}]^{+}:$505.1409, found 505.1411.

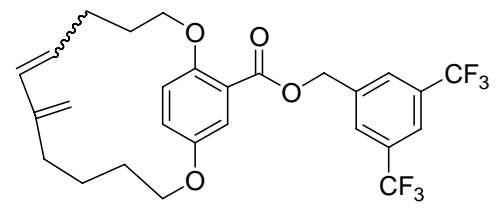

Z- 11B

${ }^{1} \mathbf{H}$ NMR $\left(\mathrm{CDCl}_{3}, 400 \mathrm{MHz}\right) \delta 7.95(\mathrm{~s}, 2 \mathrm{H}), 7.85(\mathrm{~s}, 1 \mathrm{H}), 7.49(\mathrm{~d}, J=3.0 \mathrm{~Hz}, 1 \mathrm{H}), 7.13(\mathrm{dd}, J=9.0$, $3.0 \mathrm{~Hz}, 1 \mathrm{H}), 6.99(\mathrm{~d}, J=9.0 \mathrm{~Hz}, 1 \mathrm{H}), 5.45(\mathrm{~m}, 2 \mathrm{H}), 5.20(\mathrm{~d}, J=15.7 \mathrm{~Hz}, 1 \mathrm{H}), 5.17-5.09(\mathrm{~m}, 1 \mathrm{H})$, $4.81(\mathrm{~s}, 1 \mathrm{H}), 4.60(\mathrm{~s}, 1 \mathrm{H}), 4.40-4.10(\mathrm{~m}, 4 \mathrm{H}), 2.05-1.25(\mathrm{~m}, 10 \mathrm{H}) ;{ }^{13} \mathbf{C} \mathbf{N M R}\left(\mathrm{CDCl}_{3}, 100 \mathrm{MHz}\right) \delta$ 165.6, 151.9, 150.9, 144.7, 138.9, 133.2, $132.1\left(\mathrm{q}, \mathrm{CF}_{3}\right), 128.9,127.7,124.9,123.2,122.4,121.9$, 120.9, 119.7, 113.1, 68.5, 68.3, 64.9, 30.6, 27.5, 27.0, 24.9, 22.8; HRMS (ES) Calculated for $\mathrm{C}_{27} \mathrm{H}_{27} \mathrm{~F}_{6} \mathrm{O}_{4}[\mathrm{M}+\mathrm{H}]^{+}: 529.1808$ found 529.1813. Calculated for $\mathrm{C}_{27} \mathrm{H}_{26} \mathrm{~F}_{6} \mathrm{O}_{4} \mathrm{Na}[\mathrm{M}+\mathrm{Na}]^{+}$: 551.1627, found 551.1637 .

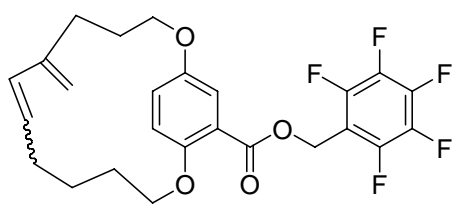

$E-13 A$

${ }^{1} \mathbf{H}$ NMR $\left(\mathrm{CDCl}_{3}, 400 \mathrm{MHz}\right) \delta 7.39(\mathrm{~d}, J=3.1 \mathrm{~Hz}, 1 \mathrm{H}), 7.04(\mathrm{dd}, J=9.0,3.1 \mathrm{~Hz}, 1 \mathrm{H}), 6.93(\mathrm{~d}, J=$ $9.0 \mathrm{~Hz}, 1 \mathrm{H}), 5.77(\mathrm{~d}, J=15.7 \mathrm{~Hz}, 1 \mathrm{H}), 5.43(\mathrm{~m}, 2 \mathrm{H}), 4.82(\mathrm{~s}, 1 \mathrm{H}), 4.78(\mathrm{~s}, 1 \mathrm{H}), 4.59-4.53(\mathrm{~m}, 1 \mathrm{H})$, 4.47- $4.38(\mathrm{~m}, 1 \mathrm{H}), 4.28-4.25(\mathrm{~m}, 3 \mathrm{H}), 1.96-1.55(\mathrm{~m}, 4 \mathrm{H}), 1.25-1.19(\mathrm{~m}, 6 \mathrm{H}) ;{ }^{13} \mathbf{C}$ NMR $\left(\mathrm{CDCl}_{3}\right.$, $100 \mathrm{MHz}) \delta 165.0,151.8,151.0,144.8,133.1,129.1,124.6,123.0,120.7,119.9,113.0,72.6,68.5$, 53.8, 32.5, 30.7, 27.4, 25.2, 22.2; HRMS (ES) Calculated for $\mathrm{C}_{25} \mathrm{H}_{24} \mathrm{~F}_{5} \mathrm{O}_{4}[\mathrm{M}+\mathrm{H}]^{+}: 483.1589$ found 483.1589. Calculated for $\mathrm{C}_{25} \mathrm{H}_{23} \mathrm{~F}_{5} \mathrm{O}_{4} \mathrm{Na}[\mathrm{M}+\mathrm{Na}]^{+}:$505.1409, found 505.1408.

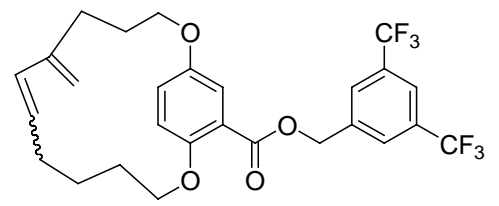

E- 15B 
${ }^{1} \mathbf{H}$ NMR $\left(\mathrm{CDCl}_{3}, 400 \mathrm{MHz}\right) \delta 7.95(\mathrm{~s}, 2 \mathrm{H}), 7.85(\mathrm{~s}, 1 \mathrm{H}), 7.47(\mathrm{~d}, J=3.0 \mathrm{~Hz}, 1 \mathrm{H}), 7.10(\mathrm{dd}, J=9.0$, $3.0 \mathrm{~Hz}, 1 \mathrm{H}), 6.96(\mathrm{~d}, J=9.0 \mathrm{~Hz}, 1 \mathrm{H}), 5.76(\mathrm{~d}, J=15.8 \mathrm{~Hz}, 1 \mathrm{H}), 5.45(\mathrm{~m}, 2 \mathrm{H}), 4.83(\mathrm{~s}, 1 \mathrm{H}), 4.79(\mathrm{~s}$, $1 \mathrm{H}), 4.61-4.49(\mathrm{~m}, 1 \mathrm{H}), 4.49-4.40(\mathrm{~m}, 1 \mathrm{H}), 4.28-4.25(\mathrm{~m}, 2 \mathrm{H}), 4.23-4.16(\mathrm{~m}, 1 \mathrm{H}), 1.96-1.60(\mathrm{~m}$, $6 \mathrm{H}), 1.32-1.25(\mathrm{~m}, 4 \mathrm{H}) ;{ }^{13} \mathbf{C}$ NMR $\left(\mathrm{CDCl}_{3}, 100 \mathrm{MHz}\right) \delta 165.6,151.9,150.9,144.7,138.9,133.2$, 132.1 (q, $\left.\mathrm{CF}_{3}\right), 128.9,127.7,124.9,123.2,122.4,121.9,120.9,119.7,113.1,68.5,68.3,64.9,30.6$, 27.5, 27.0, 24.9, 22.8; HRMS (ES) Calculated for $\mathrm{C}_{27} \mathrm{H}_{27} \mathrm{~F}_{6} \mathrm{O}_{4}[\mathrm{M}+\mathrm{H}]^{+}$: 529.1808 found 529.1803. Calculated for $\mathrm{C}_{27} \mathrm{H}_{26} \mathrm{~F}_{6} \mathrm{O}_{4} \mathrm{Na}[\mathrm{M}+\mathrm{Na}]^{+}:$551.1627, found 551.1622.

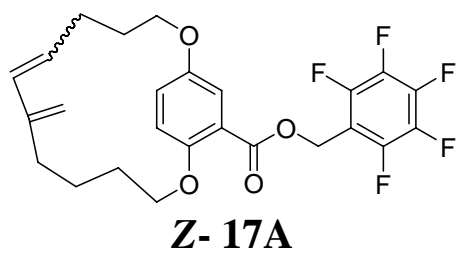

${ }^{1} \mathbf{H}$ NMR $\left(\mathrm{CDCl}_{3}, 400 \mathrm{MHz}\right) \delta 7.37(\mathrm{~d}, J=2.7 \mathrm{~Hz}, 1 \mathrm{H}), 7.06-7.02(\mathrm{~m}, 2 \mathrm{H}), 5.44(\mathrm{~d}, J=8.2 \mathrm{~Hz}, 1 \mathrm{H})$, $5.38(J=12.1 \mathrm{~Hz}, 1 \mathrm{H}), 5.30(\mathrm{~d}, J=15.7 \mathrm{~Hz}, 1 \mathrm{H}), 5.17(\mathrm{~m}, 1 \mathrm{H}), 4.82(\mathrm{~d}, J=1.5 \mathrm{~Hz}, 1 \mathrm{H}), 4.60(\mathrm{~s}$, $1 \mathrm{H}), 4.28(\mathrm{~m}, 4 \mathrm{H}), 2.24-2.05(\mathrm{~m}, 2 \mathrm{H}), 1.79-1.58(\mathrm{~m}, 4 \mathrm{H}), 1.38-1.25(\mathrm{~m}, 2 \mathrm{H}), 0.93-0.88(\mathrm{~m}, 2 \mathrm{H})$; ${ }^{13}$ C NMR $\left(\mathrm{CDCl}_{3}, 100 \mathrm{MHz}\right) \delta 165.0,154.9,153.4,145.9,131.7,128.7,123.9,122.5,122.1,120.6$, 111.3, 71.4, 70.9, 53.9, 34.6, 29.7, 28.6, 28.2, 25.7; HRMS (ES) Calculated for $\mathrm{C}_{25} \mathrm{H}_{24} \mathrm{~F}_{5} \mathrm{O}_{4}[\mathrm{M}+$ $\mathrm{H}]^{+}: 483.1589$ found 483.1581. Calculated for $\mathrm{C}_{25} \mathrm{H}_{23} \mathrm{~F}_{5} \mathrm{O}_{4} \mathrm{Na}[\mathrm{M}+\mathrm{Na}]^{+}:$505.1409, found 505.1407.

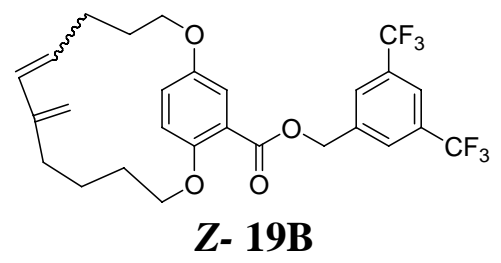

${ }^{1} \mathbf{H}$ NMR $\left(\mathrm{CDCl}_{3}, 400 \mathrm{MHz}\right) \delta 7.95(\mathrm{~s}, 2 \mathrm{H}), 7.85(\mathrm{~s}, 1 \mathrm{H}), 7.46(\mathrm{~d}, J=3.0 \mathrm{~Hz}, 1 \mathrm{H}), 7.09(\mathrm{dd}, J=9.0$, $3.0 \mathrm{~Hz}, 1 \mathrm{H}), 6.96(\mathrm{~d}, J=9.0 \mathrm{~Hz}, 1 \mathrm{H}), 5.44(\mathrm{~m}, 2 \mathrm{H}), 5.39(\mathrm{~d}, J=15.6 \mathrm{~Hz}, 1 \mathrm{H}), 5.20-5.09(\mathrm{~m}, 1 \mathrm{H})$, $4.83(\mathrm{~s}, 1 \mathrm{H}), 4.54(\mathrm{~s}, 1 \mathrm{H}), 4.28-3.90(\mathrm{~m}, 4 \mathrm{H}), 2.05-1.57(\mathrm{~m}, 4 \mathrm{H}), 1.42-1.19(\mathrm{~m}, 6 \mathrm{H}){ }^{13} \mathbf{C}$ NMR $\left(\mathrm{CDCl}_{3}, 100 \mathrm{MHz}\right) \delta 165.2,152.9,149.6,145.4,138.5,135.8,132.1\left(\mathrm{q}, \mathrm{CF}_{3}\right), 129.3,127.4,123.6$, 122.3, 121.6, 121.4, 120.3, 119.7, 111.0, 70.7, 70.6, 64.6, 29.3, 28.8, 27.9, 23.1, 22.3; HRMS (ES) Calculated for $\mathrm{C}_{27} \mathrm{H}_{27} \mathrm{~F}_{6} \mathrm{O}_{4}[\mathrm{M}+\mathrm{H}]^{+}: 529.1808$ found 529.1809. Calculated for $\mathrm{C}_{27} \mathrm{H}_{26} \mathrm{~F}_{6} \mathrm{O}_{4} \mathrm{Na}[\mathrm{M}+$ $\mathrm{Na}]^{+}:$551.1627, found 505.1623.

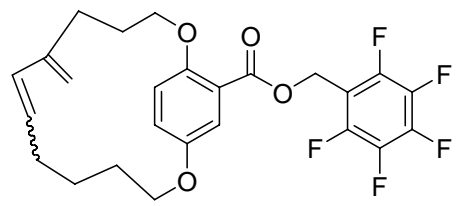

E- 21A

${ }^{1} \mathbf{H}$ NMR $\left(\mathrm{CDCl}_{3}, 400 \mathrm{MHz}\right) \delta 7.34(\mathrm{~d}, J=3.0 \mathrm{~Hz}, 1 \mathrm{H}), 7.02(\mathrm{dd}, J=9.0,3.0 \mathrm{~Hz}, 1 \mathrm{H}), 6.96(\mathrm{~d}, J=$ $9.0 \mathrm{~Hz}, 1 \mathrm{H}), 5.76(\mathrm{~d}, J=15.8 \mathrm{~Hz}, 1 \mathrm{H}), 5.43(\mathrm{~m}, 2 \mathrm{H}), 4.81(\mathrm{~s}, 1 \mathrm{H}), 4.74(\mathrm{~s}, 1 \mathrm{H}), 4.63-4.55(\mathrm{~m}, 1 \mathrm{H})$, 4.45- $4.37(\mathrm{~m}, 1 \mathrm{H}), 4.24-4.19(\mathrm{~m}, 3 \mathrm{H}), 1.96-1.80(\mathrm{~m}, 6 \mathrm{H}), 1.72-1.40(\mathrm{~m}, 2 \mathrm{H}), 1.37-1.33(\mathrm{~m}, 2 \mathrm{H})$; ${ }^{13} \mathrm{C}$ NMR $\left(\mathrm{CDCl}_{3}, 100 \mathrm{MHz}\right) \delta 165.1,152.7,150.1,144.7,133.1,129.0,124.0,122.0,121.0,119.5$, 112.9, 69.6, 67.8, 53.8, 30.8, 27.3, 26.8, 24.8, 22.8; HRMS (ES) Calculated for $\mathrm{C}_{25} \mathrm{H}_{24} \mathrm{~F}_{5} \mathrm{O}_{4}[\mathrm{M}+$ $\mathrm{H}]^{+}: 483.1589$ found 483.1593. Calculated for $\mathrm{C}_{25} \mathrm{H}_{23} \mathrm{~F}_{5} \mathrm{O}_{4} \mathrm{Na}[\mathrm{M}+\mathrm{Na}]^{+}:$505.1409, found 505.1408. 


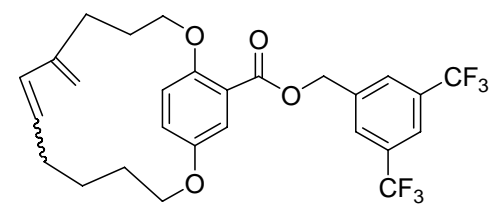

E- 23B

${ }^{1} \mathbf{H}$ NMR $\left(\mathrm{CDCl}_{3}, 400 \mathrm{MHz}\right) \delta 7.95(\mathrm{~s}, 2 \mathrm{H}), 7.85(\mathrm{~s}, 1 \mathrm{H}), 7.41(\mathrm{~d}, J=3.1 \mathrm{~Hz}, 1 \mathrm{H}), 7.05(\mathrm{dd}, J=9.0$, $3.0 \mathrm{~Hz}, 1 \mathrm{H}), 7.00(\mathrm{~d}, J=9.0 \mathrm{~Hz}, 1 \mathrm{H}), 5.77(\mathrm{~d}, J=15.9 \mathrm{~Hz}, 1 \mathrm{H}), 5.48(\mathrm{~m}, 2 \mathrm{H}), 4.80(\mathrm{~s}, 1 \mathrm{H}), 4.73(\mathrm{~s}$, $1 \mathrm{H}), 4.63-4.52(\mathrm{~m}, 1 \mathrm{H}), 4.40-4.51(\mathrm{~m}, 1 \mathrm{H}), 4.28-4.25(\mathrm{~m}, 3 \mathrm{H}), 1.96-1.79(\mathrm{~m}, 6 \mathrm{H}),, 1.60-1.52(\mathrm{~m}$, 2H), 1.42-1.36 (m, 2H); ${ }^{13} \mathbf{C}$ NMR $\left(\mathrm{CDCl}_{3}, 100 \mathrm{MHz}\right) \delta 165.7,152.6,150.2,144.6,138.9,133.1$, 132.7, $132.0\left(\mathrm{q}, \mathrm{CF}_{3}\right), 129.0,127.7,124.2,122.2,122.0,121.1,119.3,113.0,69.4,67.8,64.9,30.8$, 27.3, 26.8, 24.8, 22.8; HRMS (ES) Calculated for $\mathrm{C}_{27} \mathrm{H}_{27} \mathrm{~F}_{6} \mathrm{O}_{4}[\mathrm{M}+\mathrm{H}]^{+}: 529.1808$ found 529.1808. Calculated for $\mathrm{C}_{27} \mathrm{H}_{26} \mathrm{~F}_{6} \mathrm{O}_{4} \mathrm{Na}[\mathrm{M}+\mathrm{Na}]^{+}:$551.1627, found 551.1607.

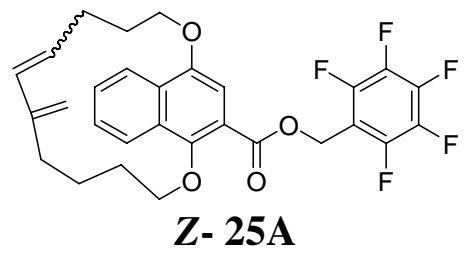

${ }^{1} \mathbf{H}$ NMR $\left(\mathrm{CDCl}_{3}, 400 \mathrm{MHz}\right) \delta 8.32(\mathrm{~d}, J=8.4 \mathrm{~Hz}, 1 \mathrm{H}), 8.15(\mathrm{~d}, J=8.2 \mathrm{~Hz}, 1 \mathrm{H}), 7.60-7.54(\mathrm{~m}, 2 \mathrm{H})$, $7.20(\mathrm{~s}, 1 \mathrm{H}), 5.48(\mathrm{~m}, 2 \mathrm{H}), 5.09(\mathrm{~m}, 1 \mathrm{H}), 4.92(\mathrm{~d}, J=15.9 \mathrm{~Hz}, 1 \mathrm{H}), 4.56(\mathrm{~d}, J=1.5 \mathrm{~Hz}, 1 \mathrm{H}), 4.50-$ $4.43(\mathrm{~m}, 2 \mathrm{H}), 4.40(\mathrm{~s}, 1 \mathrm{H}), 4.22-4.06(\mathrm{~m}, 2 \mathrm{H}), 2.17-2.03(\mathrm{~m}, 2 \mathrm{H}), 1.67-1.53(\mathrm{~m}, 2 \mathrm{H}), 1.24-1.10$ $(\mathrm{m}, 4 \mathrm{H}), 0.50(\mathrm{~m}, 1 \mathrm{H}), 0.07(\mathrm{~m}, 1 \mathrm{H}) ;{ }^{13} \mathbf{C} \mathbf{~ N M R}\left(\mathrm{CDCl}_{3}, 100 \mathrm{MHz}\right) \delta 165.8,152.0,151.5,150.2$, 145.7, 131.7, 129.8, 128.9, 128.2, 126.7, 124.4, 121.8, 116.9, 110.9, 108.6, 75.0, 70.2, 54.0, 35.0, 30.4, 28.3, 27.7, 27.5; HRMS (ES) Calculated for $\mathrm{C}_{29} \mathrm{H}_{26} \mathrm{~F}_{5} \mathrm{O}_{4}[\mathrm{M}+\mathrm{H}]^{+}: 533.1745$ found 533,1729. Calculated for $\mathrm{C}_{29} \mathrm{H}_{25} \mathrm{~F}_{5} \mathrm{O}_{5} \mathrm{Na}[\mathrm{M}+\mathrm{Na}]^{+}:$555.1571, found 555.1538.

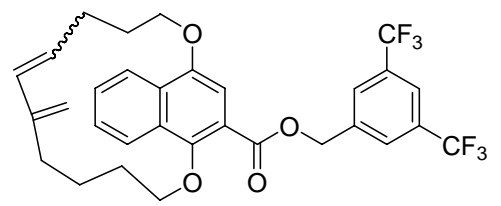

Z- 27B

${ }^{1} \mathrm{H}$ NMR $\left(\mathrm{CDCl}_{3}, 400 \mathrm{MHz}\right) \delta 8.39(\mathrm{~d}, J=8.3 \mathrm{~Hz}, 1 \mathrm{H}), 8.30(\mathrm{~d}, J=8.2 \mathrm{~Hz}, 1 \mathrm{H}), 8.01(\mathrm{~s}, 2 \mathrm{H}), 7.90$ $(\mathrm{s}, 1 \mathrm{H}), 7.65-7.59(\mathrm{~m}, 2 \mathrm{H}), 7.29(\mathrm{~m}, 1 \mathrm{H}), 5.60-5.53(\mathrm{~m}, 2 \mathrm{H}), 5.20-5.10(\mathrm{~m}, 1 \mathrm{H}), 4.98(\mathrm{~d}, J=15.6$ $\mathrm{Hz}, 1 \mathrm{H}), 4.59(\mathrm{~s}, 1 \mathrm{H}), 4.52-4.44(\mathrm{~m}, 2 \mathrm{H}), 4.41(\mathrm{~s}, 1 \mathrm{H}), 4.20-4.00(\mathrm{~m}, 2 \mathrm{H}), 2.28-1.21(\mathrm{~m}, 9 \mathrm{H}), 0.75-$ $0.46(\mathrm{~m}, 1 \mathrm{H}) ;{ }^{13} \mathbf{C} \mathbf{~ N M R}\left(\mathrm{CDCl}_{3}, 100 \mathrm{MHz}\right) \delta 165.9,151.5,145.6,138.8,132.1,131.2\left(\mathrm{q}, \mathrm{CF}_{3}\right)$, $130.8,129.8,128.9,128.2,128.0,126.7,126.6,124.5,123.9,122.1,121.8,111.5,108.3,103.9,76.7$, 70.0, 65.0, 34.9, 30.3, 28.3, 27.6, 24.0; HRMS (ES) Calculated for $\mathrm{C}_{31} \mathrm{H}_{29} \mathrm{~F}_{6} \mathrm{O}_{4}[\mathrm{M}+\mathrm{H}]^{+}$: 579.1965, found 579.1970. Calculated for $\mathrm{C}_{31} \mathrm{H}_{28} \mathrm{~F}_{6} \mathrm{O}_{4} \mathrm{Na}[\mathrm{M}+\mathrm{Na}]^{+}$: 601.1784, found 601.1788.

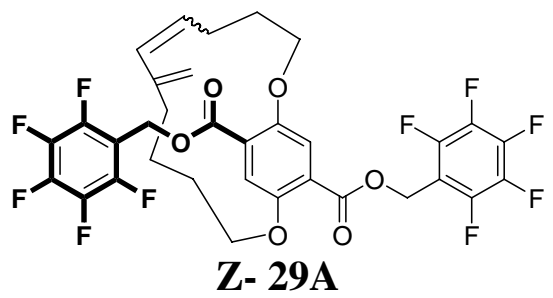


${ }^{1} \mathbf{H}$ NMR $\left(\mathrm{CDCl}_{3}, 400 \mathrm{MHz}\right) \delta 7.46(\mathrm{~s}, 1 \mathrm{H}), \delta 7.39(\mathrm{~s}, 1 \mathrm{H}), 5.43(\mathrm{~m}, 4 \mathrm{H}), 5.30(\mathrm{~m}, 2 \mathrm{H}), 4.77(\mathrm{~d}, J=$ $1.4 \mathrm{~Hz}, 1 \mathrm{H}), 4.57(\mathrm{~s}, 1 \mathrm{H}), 4.50-4.00(\mathrm{~m}, 4 \mathrm{H}), 2.21-1.95(\mathrm{~m}, 2 \mathrm{H}), 1.82-1.30(\mathrm{~m}, 4 \mathrm{H}), 0.98-0.68(\mathrm{~m}$, $4 \mathrm{H}) ;{ }^{13} \mathrm{C}$ NMR $\left(\mathrm{CDCl}_{3}, 100 \mathrm{MHz}\right) \delta 164.4,164.2,154.3,152.1,145.5,131.7,128.6,126.5,125.1$, 123.8, 121.1, 113.3, 111.5, 71.9, 70.5, 53.4, 34.4, 29.6, 29.3, 28.1, 25.5; HRMS (ES) Calculated for $\mathrm{C}_{33} \mathrm{H}_{25} \mathrm{~F}_{10} \mathrm{O}_{6}[\mathrm{M}+\mathrm{H}]^{+}:$707.1486 found 707.1489. Calculated for $\mathrm{C}_{33} \mathrm{H}_{24} \mathrm{~F}_{10} \mathrm{O}_{6} \mathrm{Na}[\mathrm{M}+\mathrm{Na}]^{+}$: 729.1311, found 729.1319 .

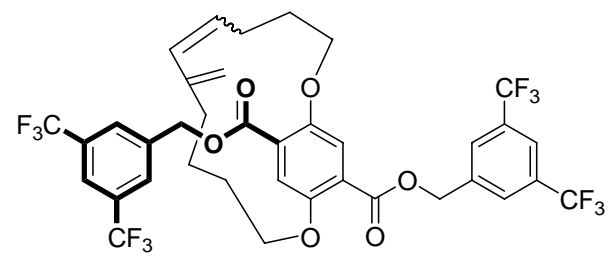

E- 31B

${ }^{1} \mathbf{H}$ NMR $\left(\mathrm{CDCl}_{3}, 400 \mathrm{MHz}\right) \delta 7.92(\mathrm{~s}, 4 \mathrm{H}), 7.87(\mathrm{~s}, 2 \mathrm{H}), 7.51(\mathrm{~s}, 1 \mathrm{H}), 7.48(\mathrm{~s}, 1 \mathrm{H}), 5.70(\mathrm{~d}, J=15.2$ $\mathrm{Hz}, 1 \mathrm{H}), 5.53-5.37(\mathrm{~m}, 4 \mathrm{H}), 4.93-4.82(\mathrm{~m}, 1 \mathrm{H}), 4.72(\mathrm{~s}, 1 \mathrm{H}), 4.63(\mathrm{~s}, 1 \mathrm{H}), 4.43-4.00(\mathrm{~m}, 4 \mathrm{H}), 2.25-$ $0.88(\mathrm{~m}, 10 \mathrm{H}) ;{ }^{13} \mathrm{C}$ NMR $\left(\mathrm{CDCl}_{3}, 100 \mathrm{MHz}\right) \delta 165.5,165.3,152.0,150.7,145.9,143.0,138.4$, 138.4, 132.6, 132.3, 132.2, 131.8 (q, $\mathrm{CF}_{3}$ ), 129.6, 128.7, 127.8, 125.8, 124.5, 122.1, 122.0, 121.8, 121.3, 120.9, 113.6, 79.0, 68.7, 65.8, 36.3.0, 31.9, 29.5, 27.9, 24.0; HRMS (ES) Calculated for $\mathrm{C}_{37} \mathrm{H}_{31} \mathrm{~F}_{12} \mathrm{O}_{6}[\mathrm{M}+\mathrm{H}]^{+}:$799.1924, found 799.1924. Calculated for $\mathrm{C}_{37} \mathrm{H}_{30} \mathrm{~F}_{12} \mathrm{O}_{6} \mathrm{Na}[\mathrm{M}+\mathrm{Na}]^{+}$: 821.1743 , found 821.1742 .

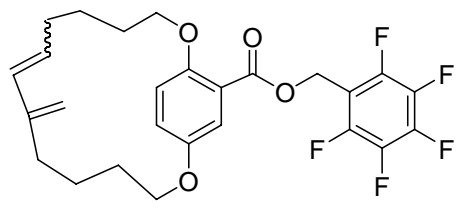

$E-33 A$

${ }^{1} \mathbf{H}$ NMR $\left(\mathrm{CDCl}_{3}, 400 \mathrm{MHz}\right) \delta 7.35(\mathrm{~d}, J=3.1 \mathrm{~Hz}, 1 \mathrm{H}), 7.07(\mathrm{dd}, J=9.0,3.1 \mathrm{~Hz}, 1 \mathrm{H}), 6.93(\mathrm{~d}, J=$ $9.1 \mathrm{~Hz}, 1 \mathrm{H}), 5.71(\mathrm{~d}, J=15.7 \mathrm{~Hz}, 1 \mathrm{H}), 5.41(\mathrm{~s}, 2 \mathrm{H}), 4.90(\mathrm{~m}, 1 \mathrm{H}), 4.77(\mathrm{~s}, 1 \mathrm{H}), 4.73(\mathrm{~s}, 1 \mathrm{H}), 4.28-$ $4.17(\mathrm{~m}, 4 \mathrm{H}), 1.93-185(\mathrm{~m}, 4 \mathrm{H}), 1.70-1.38(\mathrm{~m}, 4 \mathrm{H}), 1.30-1.21(\mathrm{~m}, 4 \mathrm{H}) ;{ }^{13} \mathbf{C} \mathbf{~ N M R}\left(\mathrm{CDCl}_{3}, 100\right.$ $\mathrm{MHz}) \delta 165.1,152.3,151.2,146.0,132.6,129.2,123.2,121.8,120.3,118.7,113.4,69.8,68.6,53.8$, 32.3, 31.7, 29.9, 26.1, 25.4, 23.9; HRMS (ES) Calculated for $\mathrm{C}_{26} \mathrm{H}_{26} \mathrm{~F}_{5} \mathrm{O}_{4}[\mathrm{M}+\mathrm{H}]^{+}: 497.1745$ found 497.1739. Calculated for $\mathrm{C}_{26} \mathrm{H}_{25} \mathrm{~F}_{5} \mathrm{O}_{4} \mathrm{Na}[\mathrm{M}+\mathrm{Na}]^{+}$519.1571, found 519.1568.

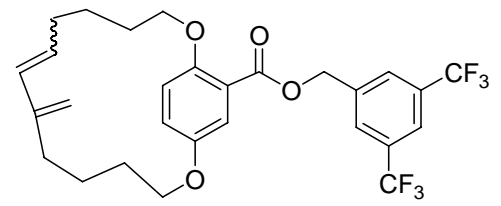

E- 35B

${ }^{1} \mathbf{H}$ NMR $\left(\mathrm{CDCl}_{3}, 400 \mathrm{MHz}\right) \delta 7.95(\mathrm{~s}, 2 \mathrm{H}), 7.85(\mathrm{~s}, 1 \mathrm{H}), 7.44(\mathrm{~d}, J=3.0 \mathrm{~Hz}, 1 \mathrm{H}), 7.10(\mathrm{dd}, J=8.9$, $3.0 \mathrm{~Hz}, 1 \mathrm{H}), 6.97(\mathrm{~d}, J=9.0 \mathrm{~Hz}, 1 \mathrm{H}), 5.72(\mathrm{~d}, J=15.7 \mathrm{~Hz}, 1 \mathrm{H}), 5.47(\mathrm{~s}, 2 \mathrm{H}), 4.93-4.85(\mathrm{~m}, 1 \mathrm{H})$, $4.78(\mathrm{~s}, 1 \mathrm{H}), 4.74(\mathrm{~s}, 1 \mathrm{H}), 4.35-4.23(\mathrm{~m}, 4 \mathrm{H}), 2.08-1.86(\mathrm{~m}, 4 \mathrm{H}), 1.80-1.21(\mathrm{~m}, 8 \mathrm{H}) ;{ }^{13} \mathbf{C}$ NMR $\left(\mathrm{CDCl}_{3}, 100 \mathrm{MHz}\right) \delta 165.7,152.3,151.1,146.0,138.9,132.6,132.0\left(\mathrm{q}, \mathrm{CF}_{3}\right), 129.1,127.7,124.6$, $123.4,121.9,121.8,120.4,118.6,113.6,69.8,68.5,64.9,32.2,31.6,29.7,26.0,25.4,23.9$; HRMS 
(ES) Calculated for $\mathrm{C}_{28} \mathrm{H}_{29} \mathrm{~F}_{6} \mathrm{O}_{4}[\mathrm{M}+\mathrm{H}]^{+}:$543.1965, found 543.1965. Calculated for $\mathrm{C}_{28} \mathrm{H}_{28} \mathrm{~F}_{6} \mathrm{O}_{4} \mathrm{Na}$ $[\mathrm{M}+\mathrm{Na}]^{+}:$565.1784, found 565.1784 .

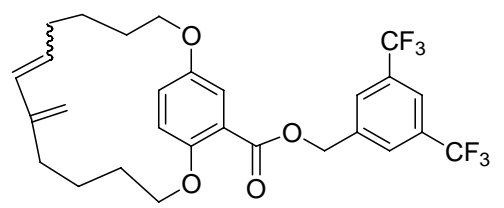

E- 37B

${ }^{1} \mathbf{H}$ NMR $\left(\mathrm{CDCl}_{3}, 400 \mathrm{MHz}\right) \delta 7.94(\mathrm{~s}, 2 \mathrm{H}), 7.85(\mathrm{~s}, 1 \mathrm{H}), 7.40(\mathrm{~d}, J=2.9 \mathrm{~Hz}, 1 \mathrm{H}), 7.06(\mathrm{dd}, J=8.9$, $2.9 \mathrm{~Hz}, 1 \mathrm{H}), 7.03(\mathrm{~d}, J=9.0 \mathrm{~Hz}, 1 \mathrm{H}), 5.73(\mathrm{~d}, J=15.7 \mathrm{~Hz}, 1 \mathrm{H}), 5.45(\mathrm{~s}, 2 \mathrm{H}), 4.99-4.89(\mathrm{~m}, 1 \mathrm{H})$, $4.73(\mathrm{~s}, 1 \mathrm{H}), 4.68(\mathrm{~s}, 1 \mathrm{H}), 4.33-4.24(\mathrm{~m}, 4 \mathrm{H}), 2.10-1.91(\mathrm{~m}, 2 \mathrm{H}), 1.85-1.80(\mathrm{~m}, 2 \mathrm{H}), 1.70-1.11(\mathrm{~m}$, $8 \mathrm{H}) ;{ }^{13} \mathrm{C}$ NMR $\left(\mathrm{CDCl}_{3}, 100 \mathrm{MHz}\right) \delta 165.6,153.0,150.5,145.8,138.8,132.6,131.7\left(\mathrm{q}, \mathrm{CF}_{3}\right), 129.1$, $127.7,124.5,123.4,122.1,121.9,120.3,119.6,113.5,70.9,68.0,64.9,32.2,31.7,29.7,26.2,25.2$, 24.0; HRMS (ES) Calculated for $\mathrm{C}_{28} \mathrm{H}_{29} \mathrm{~F}_{6} \mathrm{O}_{4}[\mathrm{M}+\mathrm{H}]^{+}: 543.1965$ found 543.1968. Calculated for $\mathrm{C}_{28} \mathrm{H}_{28} \mathrm{~F}_{6} \mathrm{O}_{4} \mathrm{Na}[\mathrm{M}+\mathrm{Na}]^{+}:$565.1784, found 565.1784.

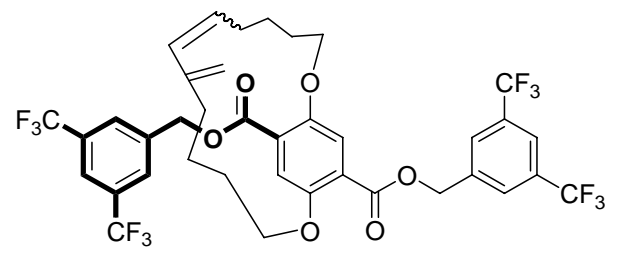

E- 39B

${ }^{1} \mathbf{H}$ NMR $\left(\mathrm{CDCl}_{3}, 400 \mathrm{MHz}\right) \delta 7.95(\mathrm{~s}, 4 \mathrm{H}), 7.87(\mathrm{~s}, 2 \mathrm{H}), 7.52(\mathrm{~s}, 1 \mathrm{H}), 7.47(\mathrm{~s}, 1 \mathrm{H}), 5.70(\mathrm{~d}, \mathrm{~J}=15.5$ $\mathrm{Hz}, 1 \mathrm{H}), 5.57-5.38(\mathrm{~m}, 4 \mathrm{H}), 4.91-4.80(\mathrm{~m}, 1 \mathrm{H}), 4.70(\mathrm{~s}, 1 \mathrm{H}), 4.65(\mathrm{~s}, 1 \mathrm{H}), 4.40-3.97(\mathrm{~m}, 4 \mathrm{H}), 2.21-$ $0.83(\mathrm{~m}, 12 \mathrm{H}) ;{ }^{13} \mathrm{C}$ NMR $\left(\mathrm{CDCl}_{3}, 100 \mathrm{MHz}\right) \delta 165.1,165.0,152.0,150.2,145.5,144.0,138.5$, $138.4,132.6,132.3,132.2,131.8$ (q, $\mathrm{CF}_{3}$ ), 129.6, 128.7, 127.8, 125.8, 124.5, 122.1, 122.0, 121.8, 120.8, 120.1, 113.5, 71.7, 68.7, 65.3, 37.0, 31.6, 29.7, 27.4, 24.6, 23.9; HRMS (ES) Calculated for $\mathrm{C}_{38} \mathrm{H}_{33} \mathrm{~F}_{12} \mathrm{O}_{6}[\mathrm{M}+\mathrm{H}]^{+}:$813.2080, found 813.2074. Calculated for $\mathrm{C}_{38} \mathrm{H}_{32} \mathrm{~F}_{12} \mathrm{O}_{6} \mathrm{Na}[\mathrm{M}+\mathrm{Na}]^{+}$: 835.1900 , found 835.1894 .

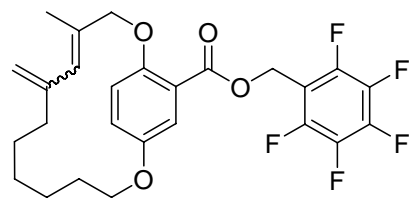

Z- 41A

${ }^{1} \mathbf{H}$ NMR $\left(\mathrm{CDCl}_{3}, 400 \mathrm{MHz}\right) \delta 7.31(\mathrm{~d}, J=3.0 \mathrm{~Hz}, 1 \mathrm{H}), 7.08(\mathrm{dd}, J=8.9,3.0 \mathrm{~Hz}, 1 \mathrm{H}), 6.96(\mathrm{~s}, 1 \mathrm{H})$, $6.88(\mathrm{~d}, J=9.1 \mathrm{~Hz}, 1 \mathrm{H}), 5.40(\mathrm{~m}, 2 \mathrm{H}), 4.60(\mathrm{~d}, J=1.9 \mathrm{~Hz}, 1 \mathrm{H}), 4.38(\mathrm{~s}, 1 \mathrm{H}), 4.73-4.47(\mathrm{~m}, 2 \mathrm{H})$, 4.25- $4.01(\mathrm{~m}, 2 \mathrm{H}), 2.20-1.93(\mathrm{~m}, 2 \mathrm{H}), 1.43-0.83(\mathrm{~m}, 9 \mathrm{H}), 0.45-0.35(\mathrm{~m}, 2 \mathrm{H}) ;{ }^{13} \mathbf{C ~ N M R}\left(\mathrm{CDCl}_{3}\right.$, $100 \mathrm{MHz}) \delta 164.9,152.3,151.8,145.9,133.6,132.2,128.8,126.3,123.2,118.5,112.7,73.8,67.6$, 53.7, 36.1, 29.9, 29.7, 27.5, 23.9, 15.2; HRMS (ES) Calculated for $\mathrm{C}_{26} \mathrm{H}_{26} \mathrm{~F}_{5} \mathrm{O}_{5}[\mathrm{M}+\mathrm{H}]^{+}: 497.1746$ found 497.1746. Calculated for $\mathrm{C}_{26} \mathrm{H}_{25} \mathrm{~F}_{5} \mathrm{O}_{5} \mathrm{Na}[\mathrm{M}+\mathrm{Na}]^{+}:$519.1565, found 519.1564. 


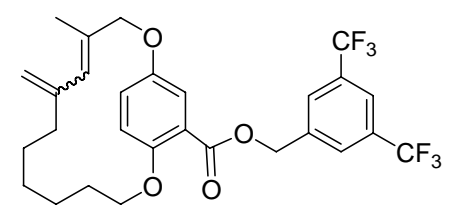

Z- 43B

${ }^{1}$ H NMR $\left(\mathrm{CDCl}_{3}, 400 \mathrm{MHz}\right) \delta 7.93$ (s, 2H), 7.85 (s, 1H), 7.37 (d, $\left.J=3.0 \mathrm{~Hz}, 1 \mathrm{H}\right), 7.08$ (dd, $J=9.1$, $3.0 \mathrm{~Hz}, 1 \mathrm{H}), 6.91(\mathrm{~d}, J=9.1 \mathrm{~Hz}, 1 \mathrm{H}), 5.72(\mathrm{~s}, 1 \mathrm{H}), 5.45-5.40(\mathrm{~m}, 2 \mathrm{H}), 4.75(\mathrm{~s}, 1 \mathrm{H}), 4.40$ (s, $1 \mathrm{H})$, 4.39 (s, 2H), 4.20- 3.99 (m, 2H), 2.25- 0.80 (m, 13H); ${ }^{13}$ C NMR $\left(\mathrm{CDCl}_{3}, 100 \mathrm{MHz}\right) \delta 165.5,152.3$, 151.9, 145.9, 139.0, 135.0, 133.6, 133.5, 132.0, 131.7 (q, $\left.\mathrm{CF}_{3}\right)$, 127.9, 127.2, 125.5, 118.4, 117.3, 112.7, 73.8, 67.4, 64.8, 36.0, 29.7, 27.3, 23.9, 21.3, 15.2; HRMS (ES) Calculated for $\mathrm{C}_{28} \mathrm{H}_{29} \mathrm{~F}_{6} \mathrm{O}_{4}$ [M $+\mathrm{H}]^{+}$: 543.1965, found 543.1964. Calculated for $\mathrm{C}_{28} \mathrm{H}_{28} \mathrm{~F}_{6} \mathrm{O}_{4} \mathrm{Na}[\mathrm{M}+\mathrm{Na}]^{+}$: 565.1784, found 565.1783.

\section{General procedure for the hydrogenation of the ene-yne metathesis products}

A general procedure for the hydrogenation of 1,3- diene paracyclophanes is detailed below. As a model, products $\mathbf{8 A}, 33 \mathrm{~A}$ and $37 \mathbf{B}$ were fully hydrogenated and the characterization data for the compounds produced are described below.

A flame dried round bottom flask, equipped with a magnetic stirrer was added the 1,3-diene product (1.0 eq.) and a mixture of ethyl acetate: $\mathrm{MeOH}(10: 1)$. The catalyst $\mathrm{Pd}(\mathrm{OH})_{2} / \mathrm{C}(10 \mathrm{~mol} \%)^{9}$ was added the sealed flask was fitted with a baloon of $\mathrm{H}_{2}(\mathrm{~g})$. The reaction mixture was stirred at room temperature for $15 \mathrm{~h}$ and the balloon of $\mathrm{H}_{2}$ (g) was typically refilled halfway through the reaction. The reaction mixture was concentrated in vacuo, dry-packed and purified by flash column silica chromatography (Hexanes-Ethyl Acetate 20:1) to afford the desired paracyclophanes. As a consequence of the presence of atropisomerism (due to the rigidity of the macrocycle) and the introduction of a stereogenic methyl group following hydrogenation, a mixture of inseparable diastereoisomers is present. Therefore, all ${ }^{1} \mathrm{H}$ and ${ }^{13} \mathrm{C}$ NMR signals are 'doubled' (see spectra for more details).

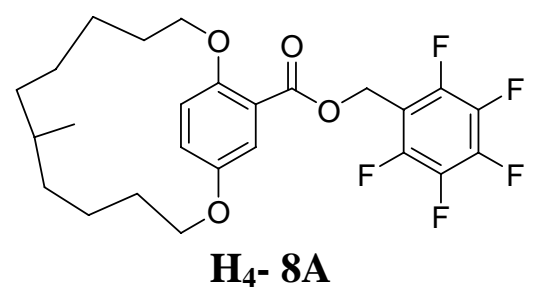

${ }^{1} \mathbf{H}$ NMR (CDCl , $\left.400 \mathrm{MHz}\right) \delta 7.40(\mathrm{~m}, 1 \mathrm{H}), 7.10(\mathrm{~m}, 1 \mathrm{H}), 7.00(\mathrm{~d}, J=9.0 \mathrm{~Hz}, 1 \mathrm{H}), 5.41(\mathrm{~m}, 2 \mathrm{H})$, 4.30- 4.13 (m, 4H), 1.90- 0.82 (br, 15H), 0.70- 0.63 (d, $J=6 \mathrm{~Hz}, 3 \mathrm{H}) ;{ }^{13} \mathbf{C}$ NMR (CDCl , $100 \mathrm{MHz}$ ) $\delta$ 165.1, 153.7, 153.2, 128.8, 121.8, 121.0, 120.3, 71.5, 69.6, 53.7, 35.5, 34.4, 31.8, 30.7, 28.4, 25.3, 23.9, 22.0, 19.6; HRMS (ES) Calculated for $\mathrm{C}_{25} \mathrm{H}_{28} \mathrm{~F}_{5} \mathrm{O}_{4}[\mathrm{M}+\mathrm{H}]^{+}: 487.1902$ found 487.1900. Calculated for $\mathrm{C}_{25} \mathrm{H}_{27} \mathrm{~F}_{5} \mathrm{O}_{4} \mathrm{Na}[\mathrm{M}+\mathrm{Na}]^{+}:$509.1721, found 509.1728.

\footnotetext{
${ }^{9}$ Other catalysts such as $\mathrm{Pd} / \mathrm{C}$ and $\mathrm{PtO}_{2}$ were not effective for the hydrogenation.
} 


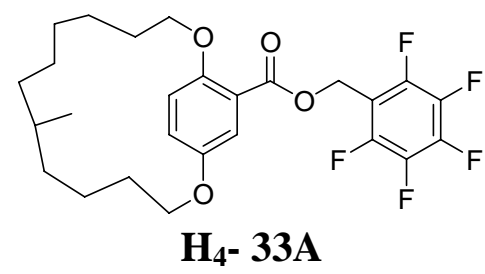

${ }^{1} \mathbf{H}$ NMR $(C D C l, 400 \mathrm{M} \mathrm{Hz}) \delta 7.34(\mathrm{~d}, \mathrm{~J}=2.8 \mathrm{~Hz}, 1 \mathrm{H}), 7.08(\mathrm{dd}, \mathrm{J}=8.9,2.8 \mathrm{~Hz}, 1 \mathrm{H}), 6.97(\mathrm{~d}, \mathrm{f}=$ $9.0 \mathrm{~Hz}, 1 \mathrm{H}), 5.42^{\prime}(\mathrm{s}, 2 \mathrm{H}), 4.28-4.14(\mathrm{br}, 4 \mathrm{H}), 1.85-1.70(\mathrm{br}, 4 \mathrm{H}), 1.28-0.75(\mathrm{br}, 13 \mathrm{H}), 0.71(\mathrm{~d}, \mathrm{~J}=$ $6.4 \mathrm{~Hz}, 3 \mathrm{H}) ;{ }^{13} \mathbf{C}$ NMR $(\mathrm{CDCl}, 100 \mathrm{M} \mathrm{Hz}) \delta 165.3,153.0,152.7,123.1,121.6,119.3,118.9,69.9$, 69.2, 53.7, 35.3, 34.3, 32.4, 29.7, 29.2, 27.7, 26.1, 24.2, 22.7, 20.3. HRMS (ES) Calculated for $\mathrm{C}_{26} \mathrm{H}_{30} \mathrm{~F}_{5} \mathrm{O}_{4}\left[\mathrm{M}+\mathrm{H}^{+}: 501.2059\right.$ found 501.2061. Calculated for $\mathrm{C}_{26} \mathrm{H}_{29} \mathrm{~F}_{5} \mathrm{O}_{4} \mathrm{Na}[\mathrm{M}+\mathrm{Na}]$ : 523.1878, found 523.1881 .

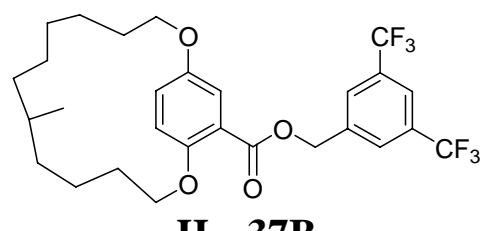

$\mathrm{H}_{4}-\mathbf{3 7 B}$

${ }^{1} \mathbf{H}$ NMR $\left(\mathrm{CDCl}_{3}, 400 \mathrm{M} \mathrm{Hz}\right) \delta 7.95(\mathrm{~s}, 2 \mathrm{H}), 7.85(\mathrm{~s}, 1 \mathrm{H}), 7.43(\mathrm{~d}, \mathrm{~J}=3.0 \mathrm{~Hz}, 1 \mathrm{H}), 7.10(\mathrm{dd}, \mathrm{J}=8.9$, $3.0 \mathrm{~Hz}, 1 \mathrm{H}), 7.02(\mathrm{~d}, \mathrm{~J}=9.0 \mathrm{~Hz}, 1 \mathrm{H}), 5.46(\mathrm{~s}, 2 \mathrm{H}), 4.29-4.22(\mathrm{~m}, 4 \mathrm{H}), 2.25-1.89(\mathrm{~m}, 2 \mathrm{H}), 1.60-$ $1.57(\mathrm{~m}, 2 \mathrm{H}), 1.38-1.20(\mathrm{~m}, 9 \mathrm{H}), 1.15-0.88(\mathrm{~m}, 4 \mathrm{H}), 0.69(\mathrm{~d}, \mathrm{~J}=6.5 \mathrm{~Hz}, 3 \mathrm{H}) ;{ }^{13} \mathbf{C} \mathbf{~ N M R}\left(\mathrm{CDCl}_{3}\right.$, $100 \mathrm{M} \mathrm{Hz}) \delta 165.8,152.2,138.8,132.6,132.0\left(\mathrm{q}, \mathrm{CF}_{3}\right), 129.7,128.8,126.6,122.0,121.5,120.4$, $118.6,70.2,68.8,64.9,35.2,34.3,29.2,29.0,27.9,24.1,22.4,22.3,20.3,14.0$; HRMS (ES) Calculated for $\mathrm{C}_{28} \mathrm{H}_{33} \mathrm{~F}_{6} \mathrm{O}_{4}[\mathrm{M}+\mathrm{H}]^{+}: 547.2278$, found 547.2279. Calculated for $\mathrm{C}_{28} \mathrm{H}_{32} \mathrm{~F}_{6} \mathrm{O}{ }_{4} \mathrm{Na}$ [M + $\mathrm{Na]^{+ }}$ : 569.0297, found 569.2097. 


\section{Results of MP2 energies and DFT calculations}

In evaluating the conformational control elements described herein, we assumed that the relative aromatic-aromatic conformational geometry in the substrates might play a crucial role in determining the product of the reaction. As such, several possible lowest energy initial geometries for each substrate were generated by a conformational search at the A M 1 level.

Subsequently, we wished to further refine the geometries and energies of the open and stacked conformers and turned to the second-order Møller-Plesset (MP2) and DFT methods. Given that recent M P2 calculations for the hexafluorobenzene:benzene complex shows that dispersion forces are important for the stabilisation of the complex, ${ }^{10}$ we first investigated M P2 level of theory in an attempt to include dispersion interactions, despite the fact that this method is very time-consuming.

However, due to the evident importance of correlation effects in the hexafluorobenzene:benzene interaction, it also seemed possible that density functional theory (DFT) could be valuable. We therefore evaluated each substrate conformer at the B $3 L Y$ P/6-31G* level. Although the results obtained from the DFT are commented on in the main text, Table S1 summarizes also the results obtained from the MP2 calculations and shows that the MP2 correlation energies produced by this level of theory follow the same tendencies as the heats of formation obtained through DFT.

Table SI1: M P2 Energies and DFT Heats of Formation.

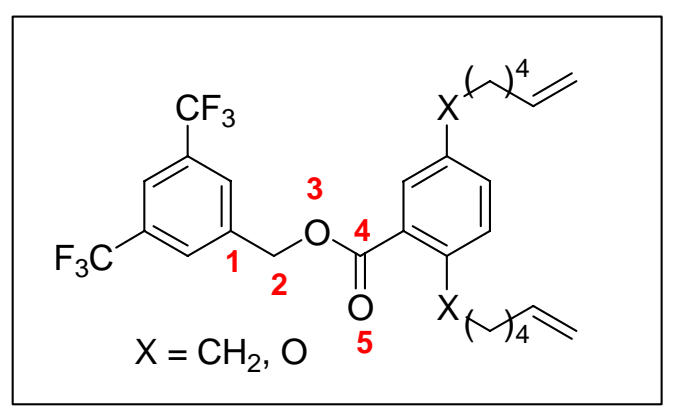

Molecule MP2 Energy $(\mathrm{kcal} / \mathrm{mol}) \quad$ DFT Heat of Formation

\begin{tabular}{ccc} 
& & $\begin{array}{c}\text { DFT } \\
(\mathrm{kcal} / \mathrm{mol})\end{array}$ \\
\hline $\mathbf{5 *}$ & $0:-899.38$ & $0:-255.7$ \\
& $\mathrm{~S}:-901.23$ & $\mathrm{~S}:-255.9$ \\
& $0:-1283.01$ & $\mathrm{O}:-351.77$ \\
$\mathbf{4 5}$ & $\mathrm{S}:-1288.29$ & $\mathrm{~S}:-353.05$ \\
& $0:-1231.43$ & $0:-207.49$ \\
$\mathbf{4 6}$ & $\mathrm{S}:-1233.01$ & $\mathrm{~S}:-208.04$ \\
& $0:-1311.27$ & $0:-309.04$ \\
& $\mathrm{~S}:-1313.08$ & $\mathrm{~S}:-309.44$ \\
\hline
\end{tabular}

10 (a) Hernandez-Trujillo, J.; Colmenares, F.; Cuevas, G.; Costas, M. Chem Phys. Lett. 1997, 265, 503. (b) Vanspeybrouck, W.; Herrebout, W. A .; van der Veken, B. J.; Lundell, J.; Perutz, R. N. J. Phys. Chem. B, 2003, 107, 13855. 
Table SI2: Dihedral Angles (as defined below)

\begin{tabular}{ccccc} 
Molecule & Dihedral angle $2(2-3-4-5)$ & \multicolumn{2}{c}{ Dihedral angle $1(1-2-3-4)$} \\
\hline $\mathbf{5}$ & $0: 30$ & $0: 29.38$ & $0: 172.17$ & $0: 173.98$ \\
& S: 165.4 & S: 171.63 & $S: 71.38$ & $S: 81.88$ \\
$\mathbf{4 4}$ & $0:-175.12$ & $0:-176.96$ & $0: 89.66$ & $0:-138.78$ \\
& $S:-177.41$ & $S:-177.43$ & $S: 72.19$ & $S: 88.41$ \\
$\mathbf{4 5}$ & $0: 40.38$ & $0: 77.16$ & $0: 167.97$ & $0: 127.89$ \\
& $S: 151.93$ & $S: 140.38$ & $S: 63.25$ & $S: 67.97$ \\
$\mathbf{4 6}$ & $0:-176.96$ & $0: 160.75$ & $0:-138.78$ & $0 *: 122.27$ \\
& $S: 162.18$ & $S:-175.18$ & $S: 83.04$ & $S: 89.66$ \\
\hline
\end{tabular}

NMR spectra of all precursors and metathesis products 


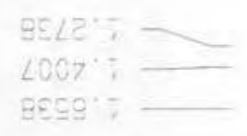

รงอย

פट87
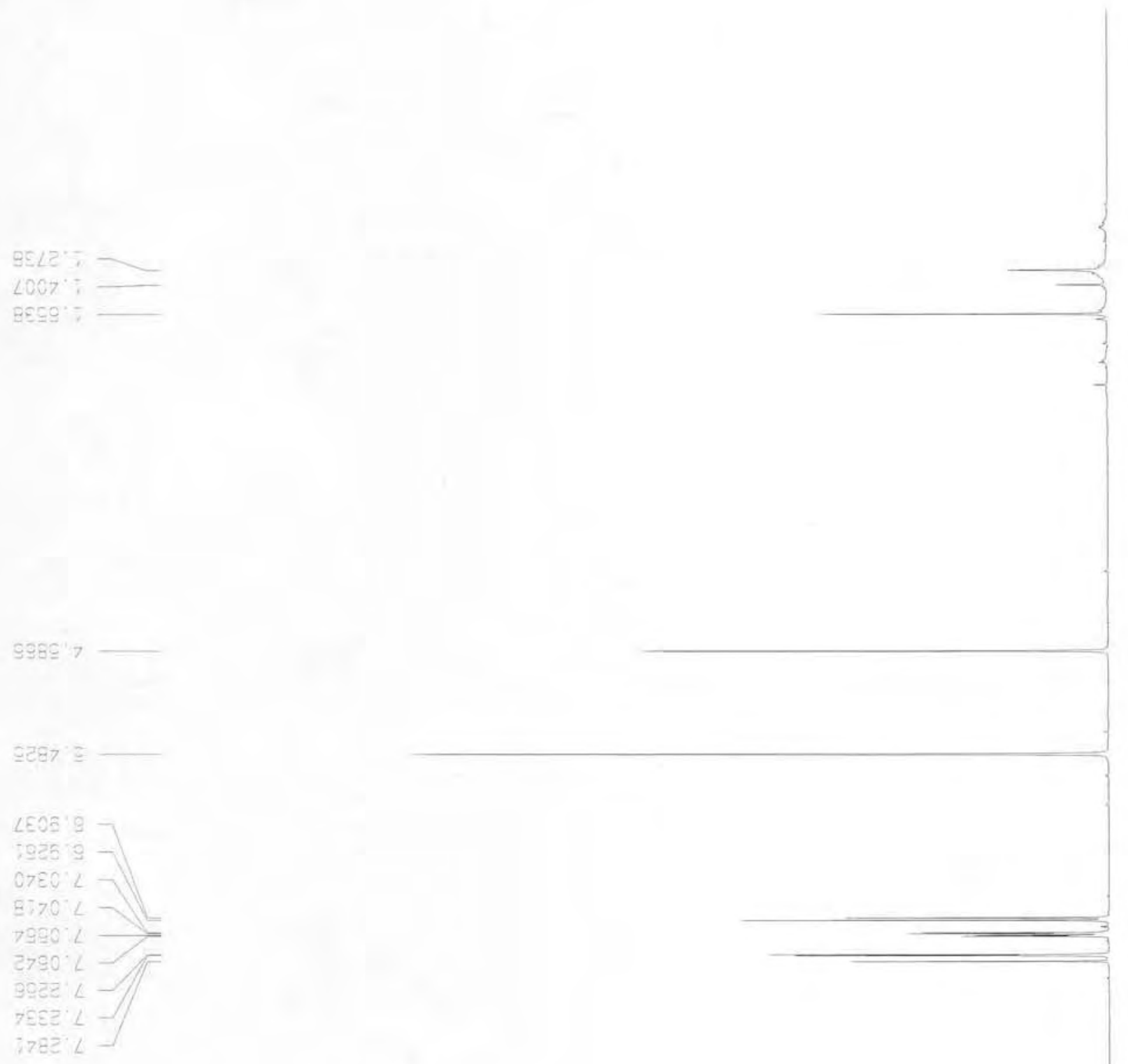
Eis, ,

LES 7::

EZL'EZ:

769* 7E:

\section{$9 \angle 9^{\circ} \angle F T$}

SEI SET

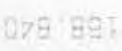

$-30$
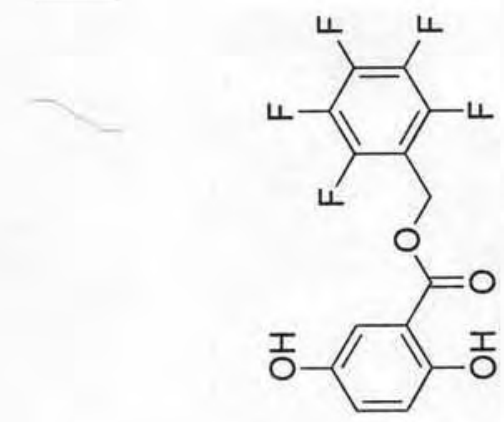

(4)
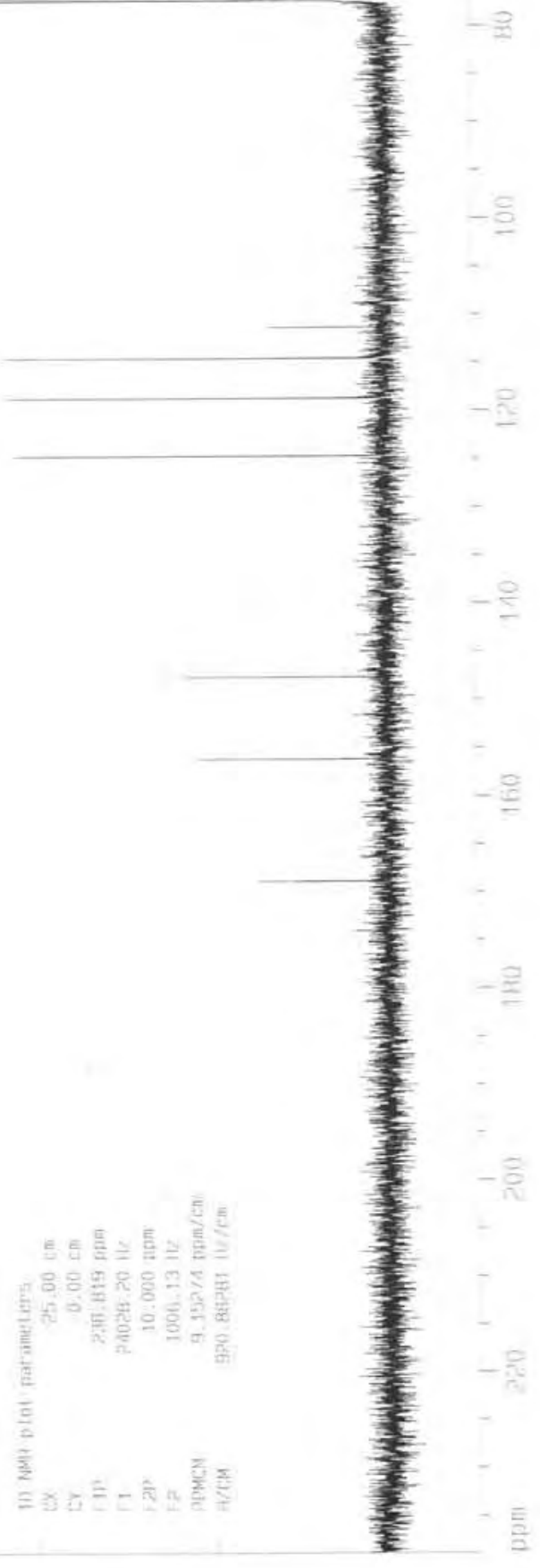
$1128 \circ$

$\angle B G V^{\circ} G$
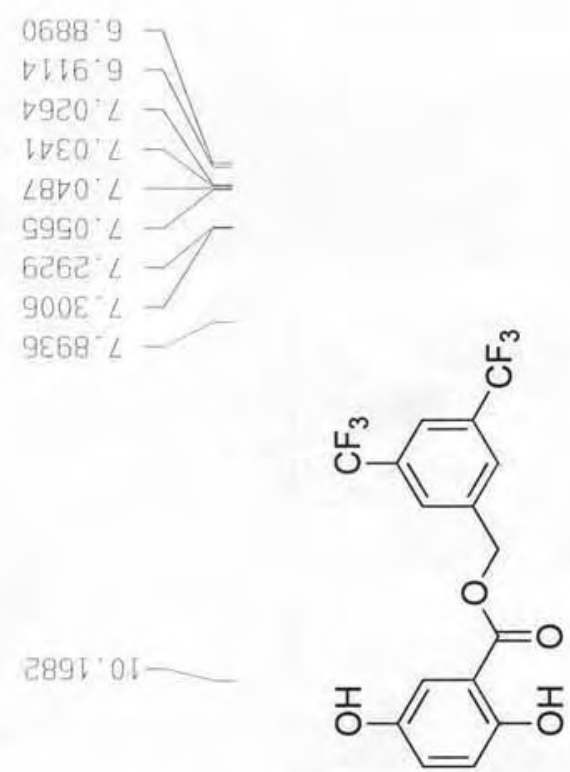

(C)

$289101-$

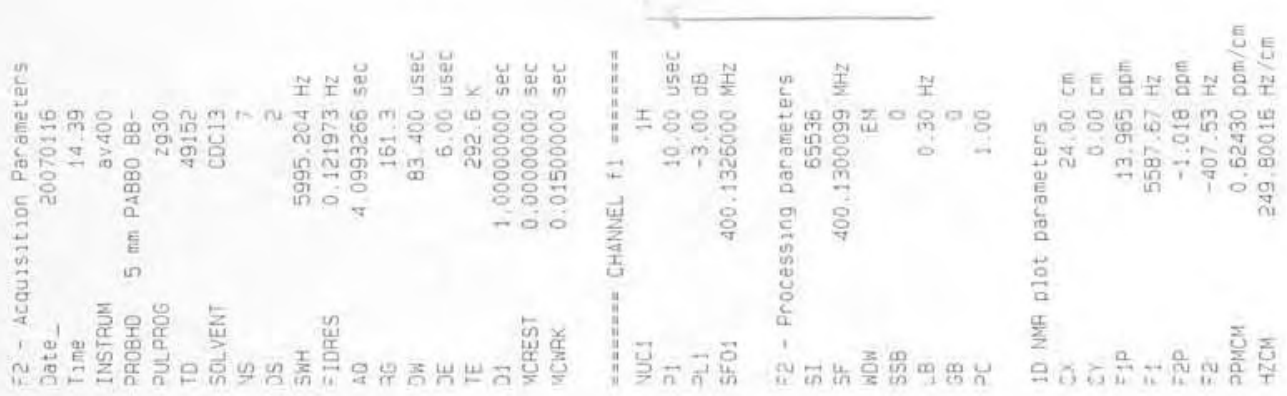




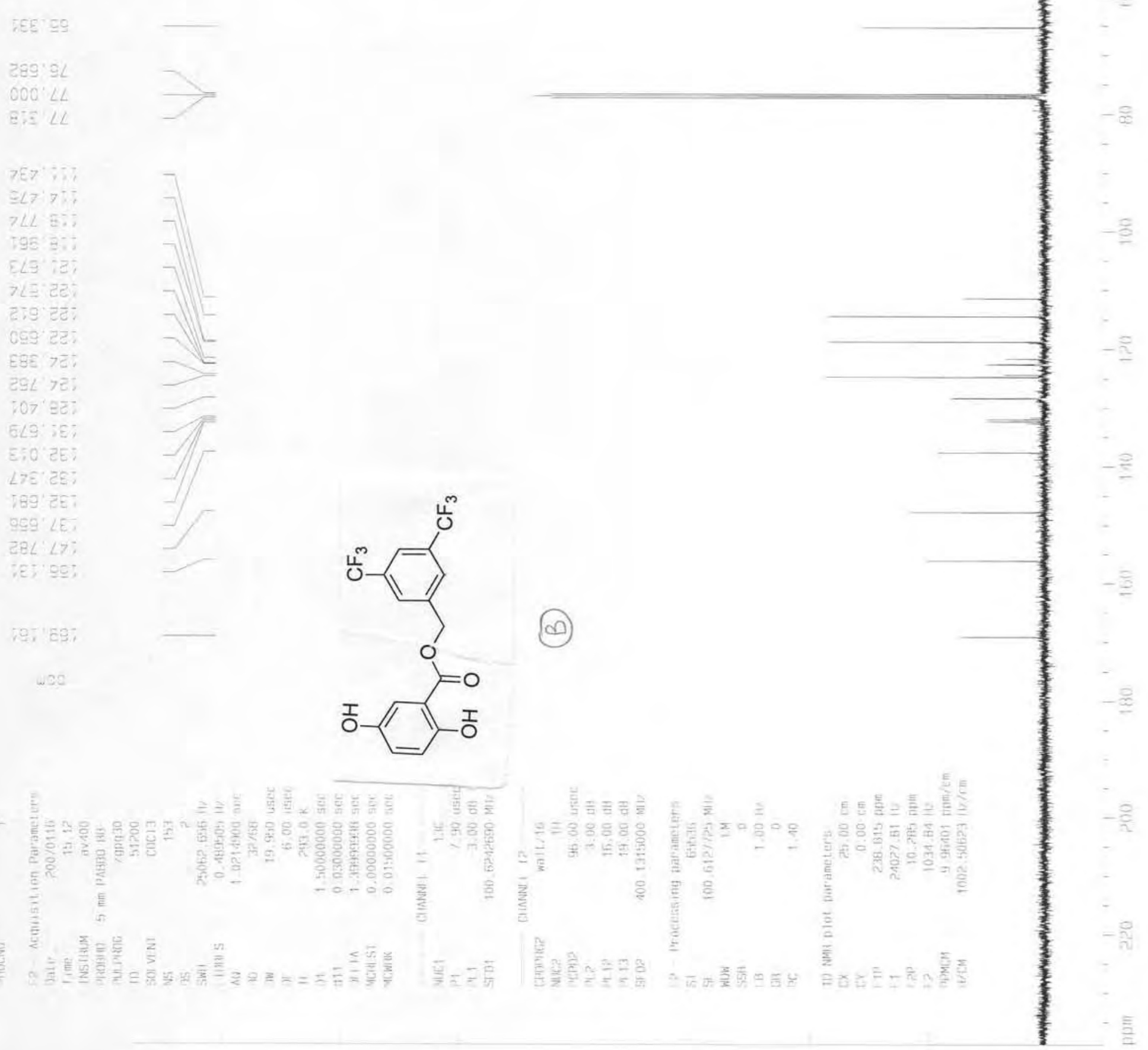




\section{GBOSE: ; \\ $\angle E L L E$ \\ $\angle 7 S 9 S$}

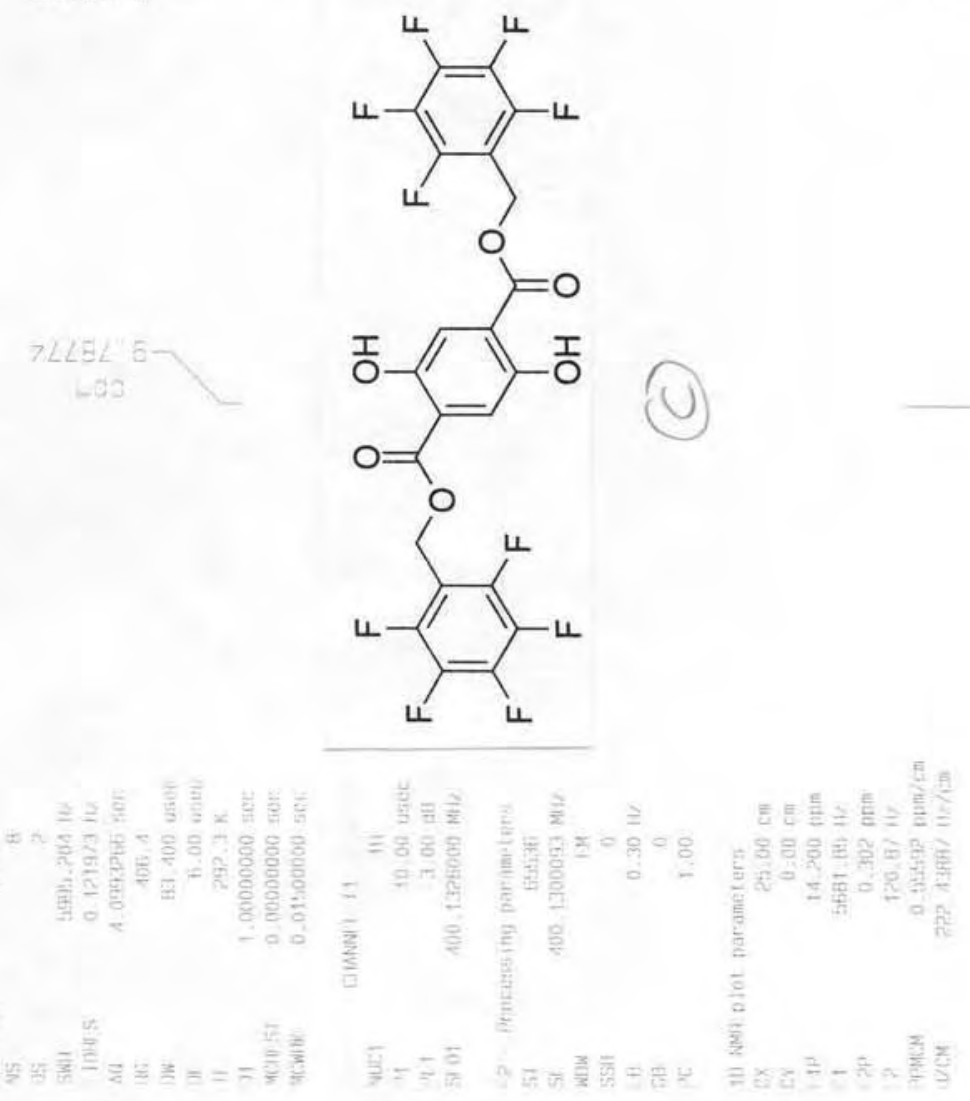


S30

LEE $7 E$

$589 \div 4$

$000 \mathrm{LL}$

Gie $\angle L$

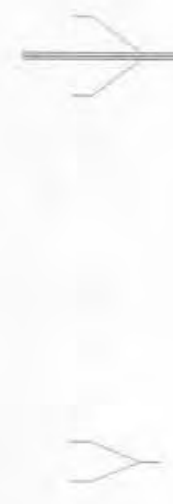

$875<1 \%$
$996 \angle 7$<smiles>CCc1c(Cl)c(CC)c(Br)c(CC)c1Cl</smiles>

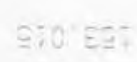

ขอเ 89

4ख0

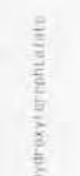<smiles>O=C(O)c1cc([O-])c(C(=O)O)cc1[O-]</smiles>

(v)<smiles>[AlH2]c1c(Cl)c(CCl)c(Cl)c(Br)c1Br</smiles>

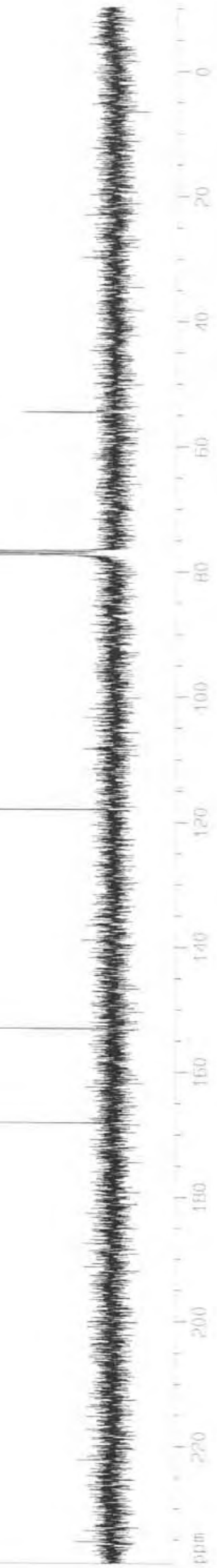




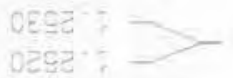

$9976>$

00572

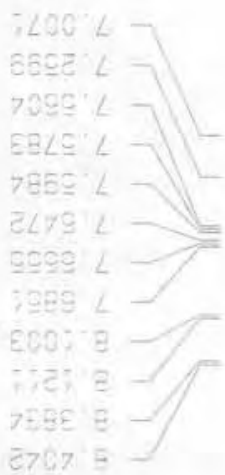

7962 :

Lez

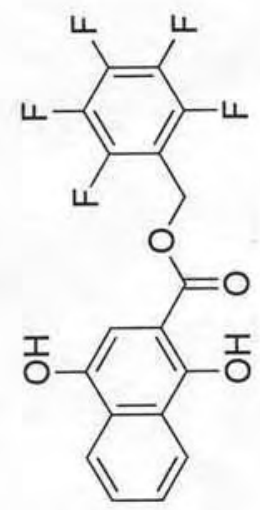

(a)
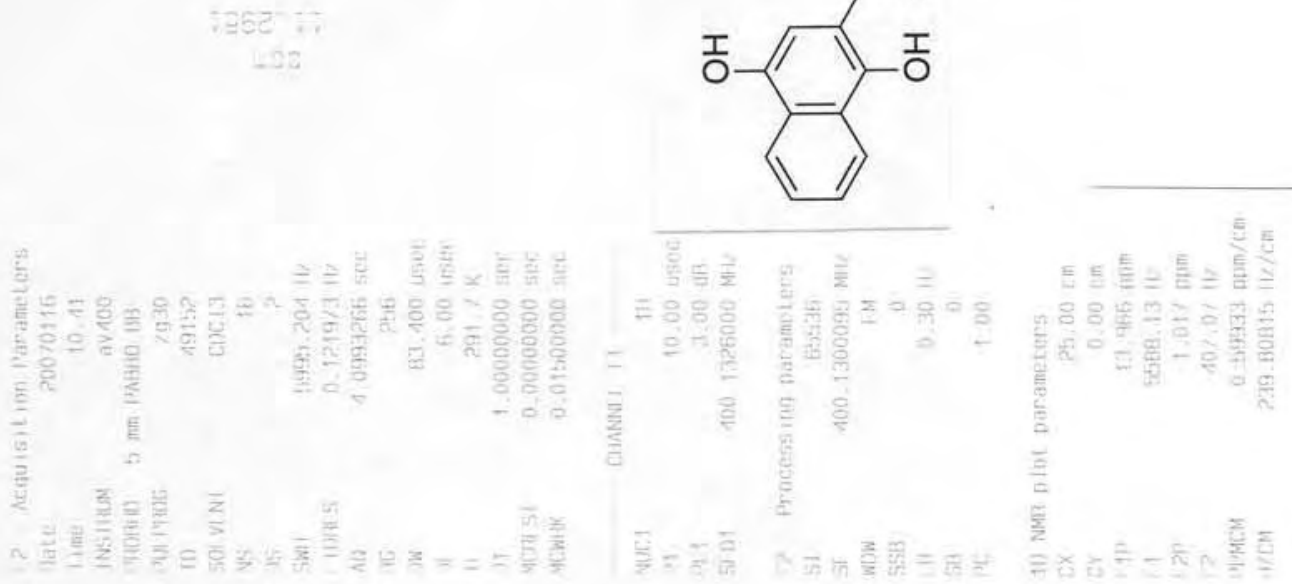


\section{DeS ET:}

det'se:

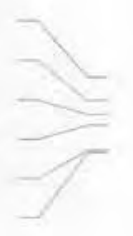

:3L 69:

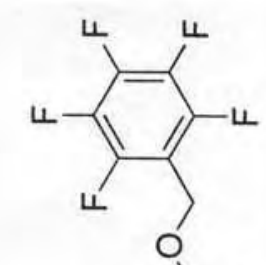

(5)

400
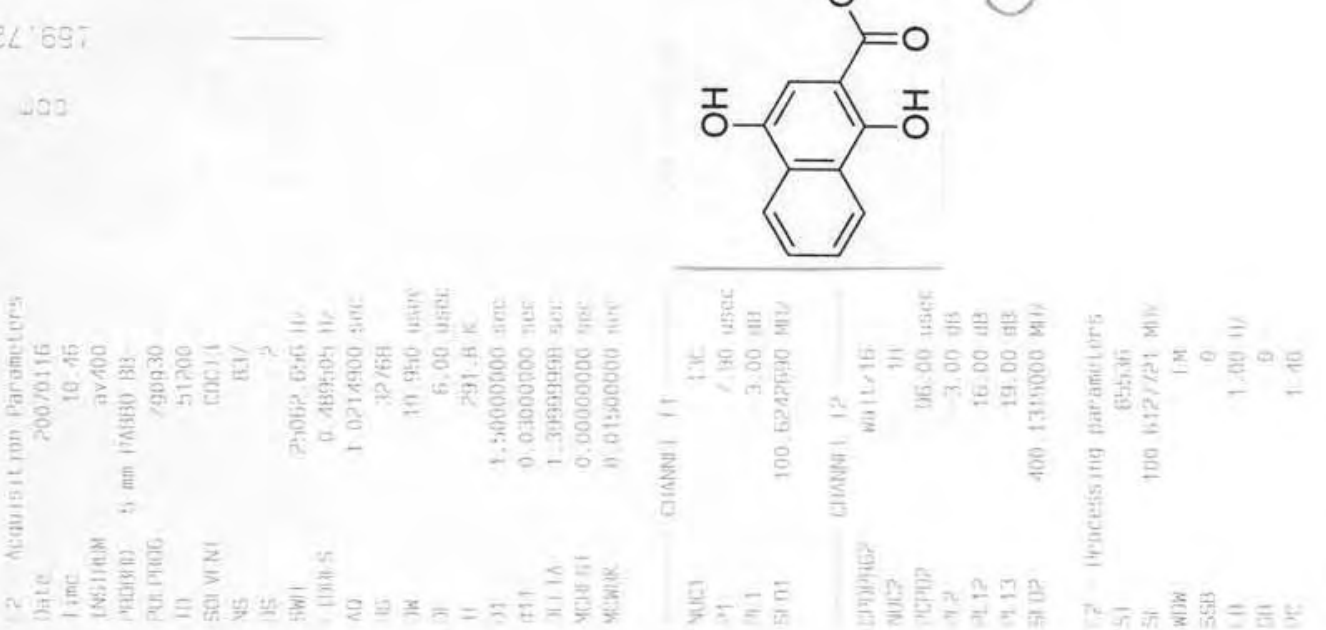

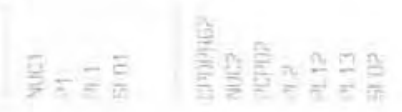

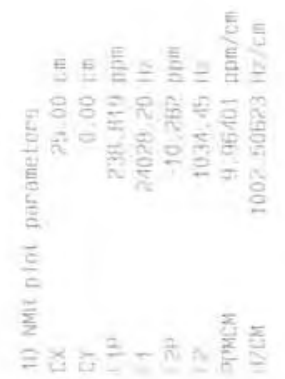




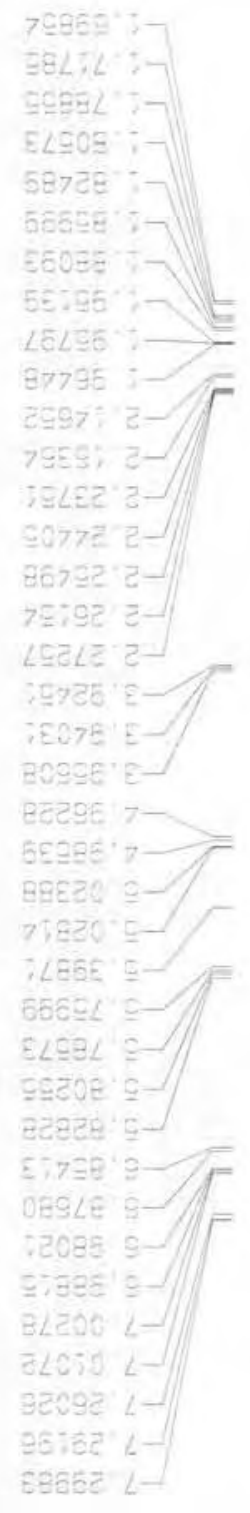

$\ldots 53$
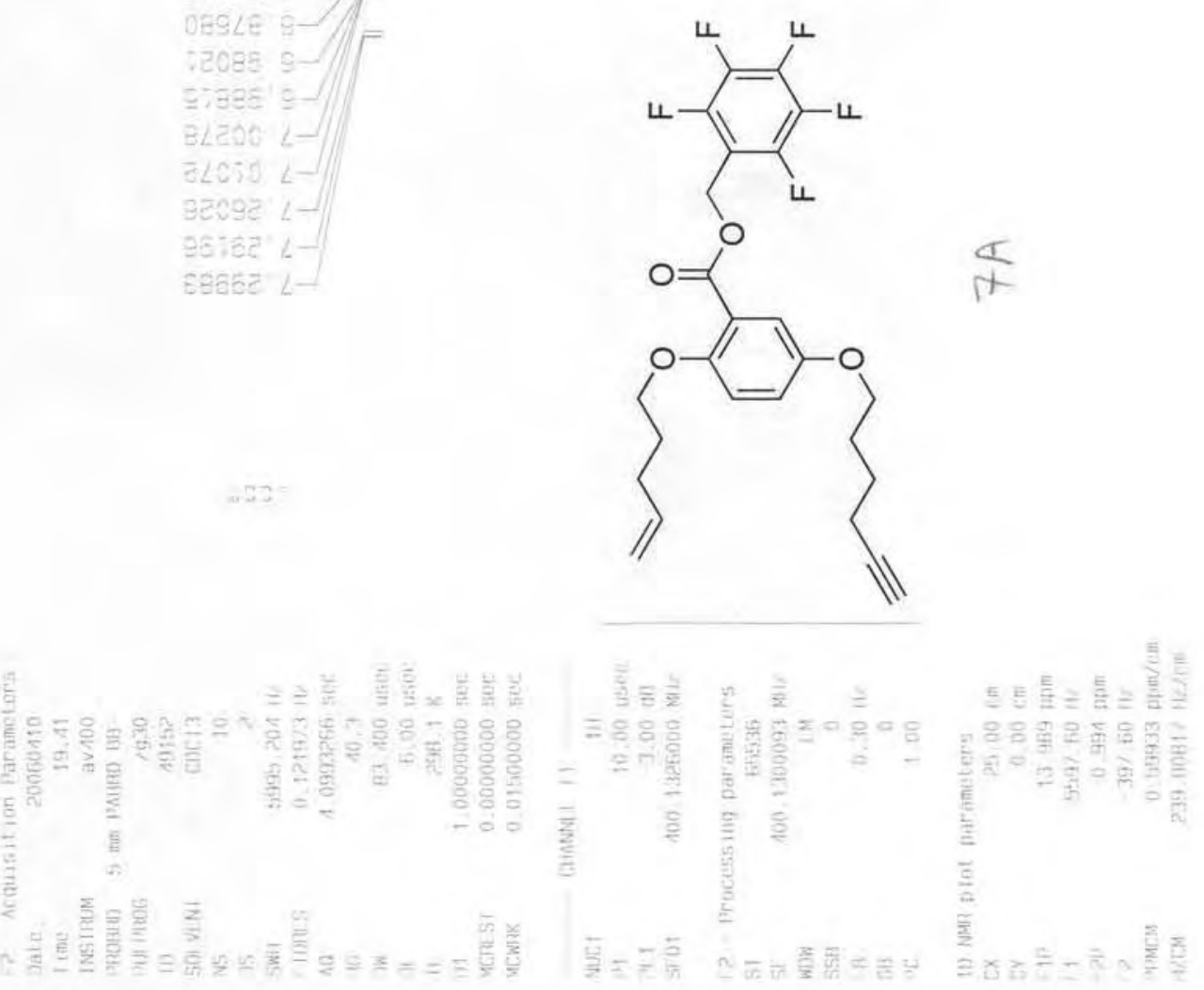
ceo a;

ज76'7ट

साद 28

GLC EZ

BER EZ

ZLG'ES

095: $\angle 9$

609 99

07899

दeg' 32

$0.00: \angle L$

gis $\angle L$

758,58

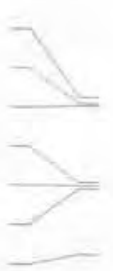

$799: 807$

$165: 7:$ :

ECO'E::

8อg:g:

C79 $51 \%$

iig'ozi

EQS $\angle E Y$

E06. 977

$798 \mathrm{ZS:}$

ESO S?:

gac ga-

400

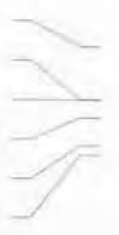

-
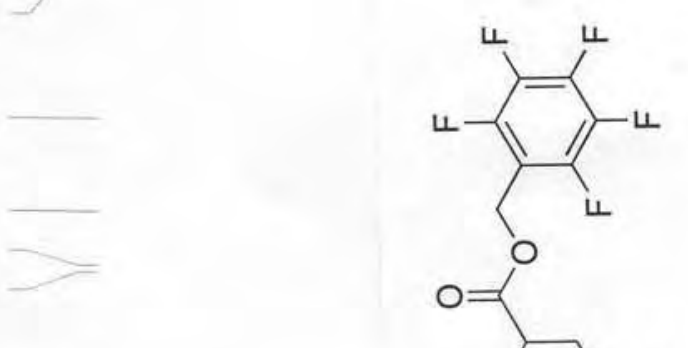

$\frac{\pi}{\pi}$
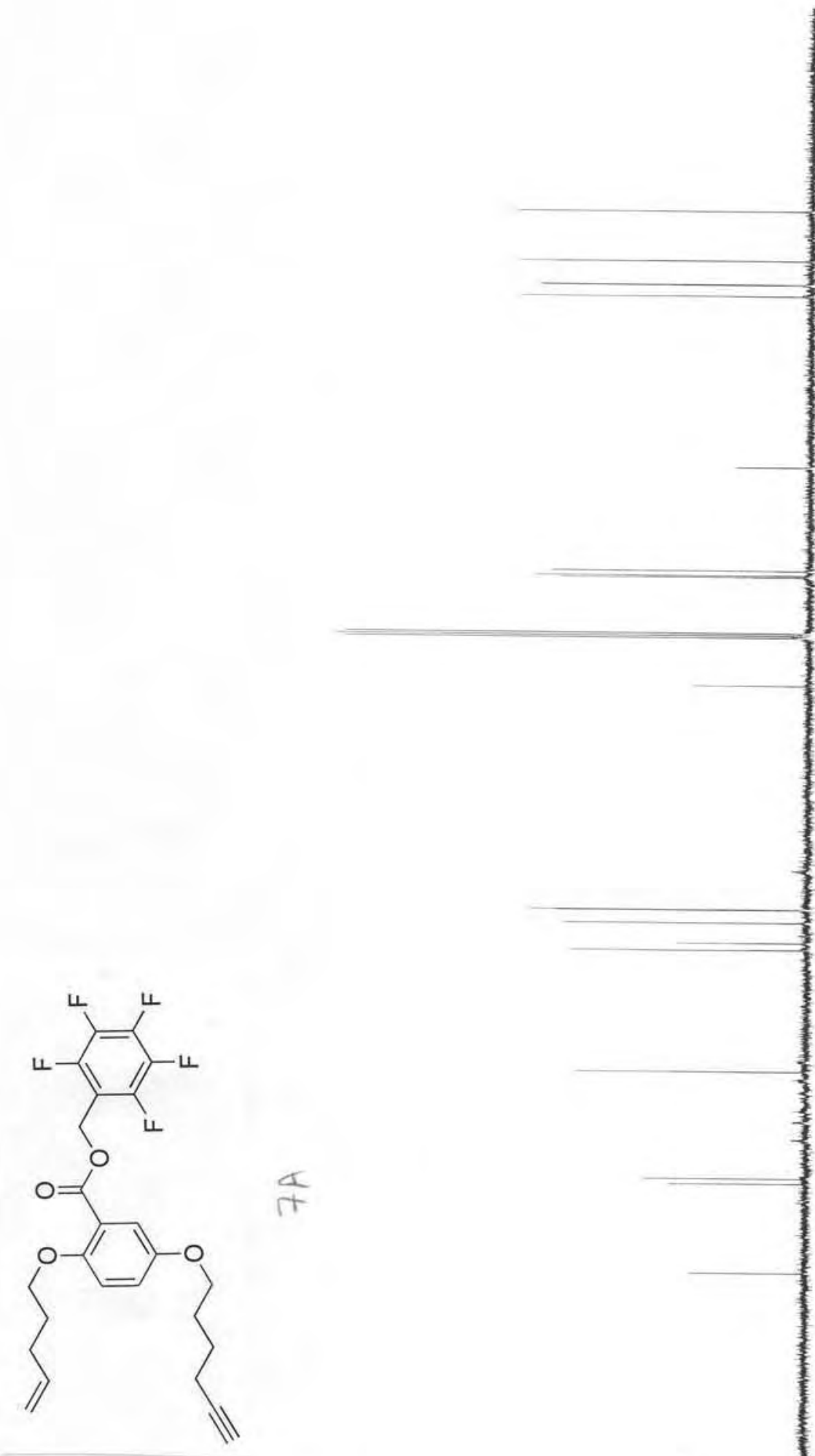

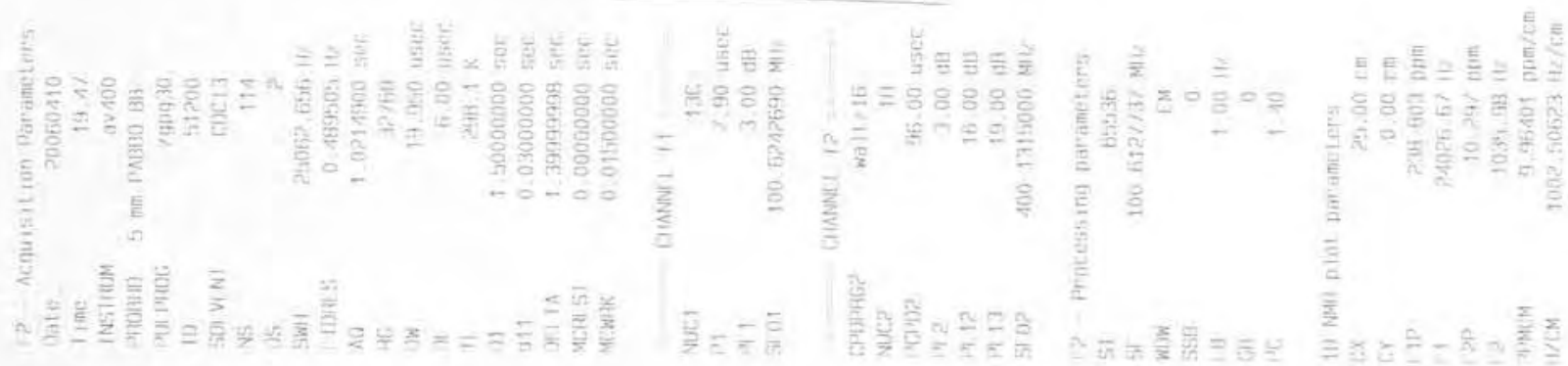




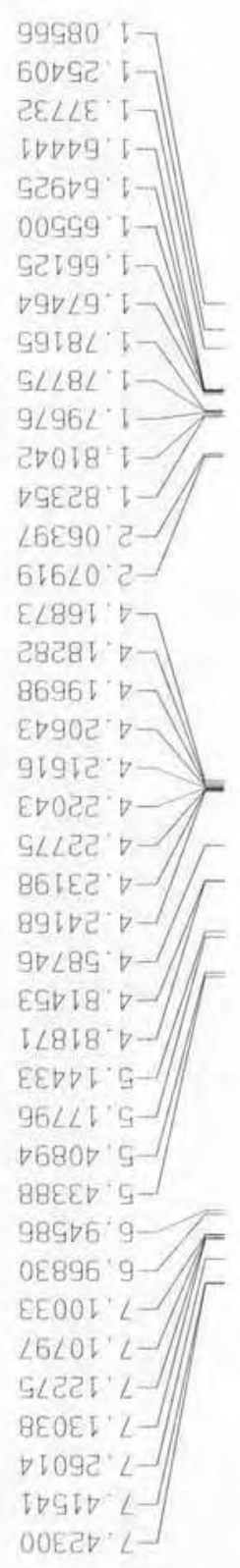

udd
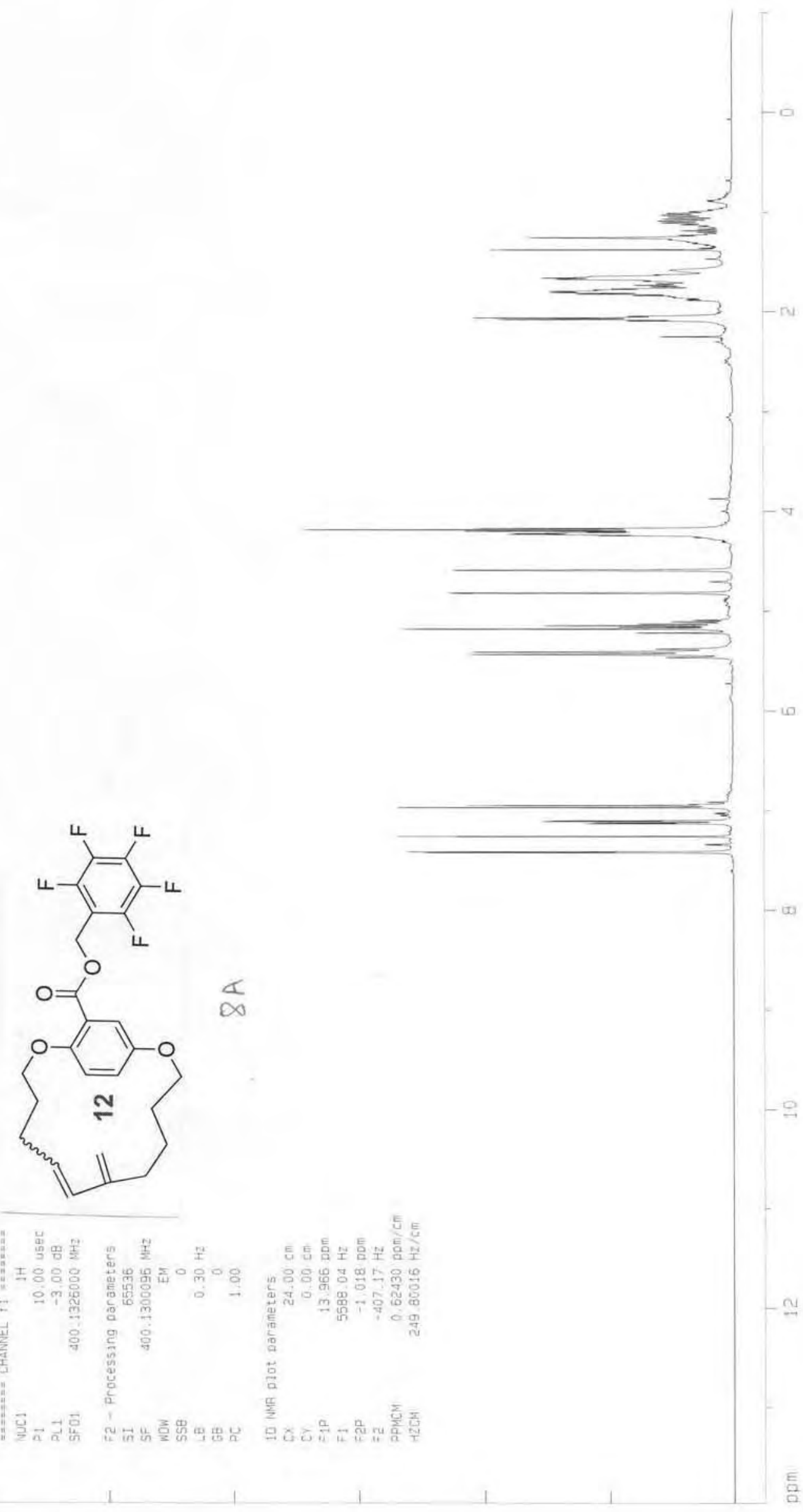

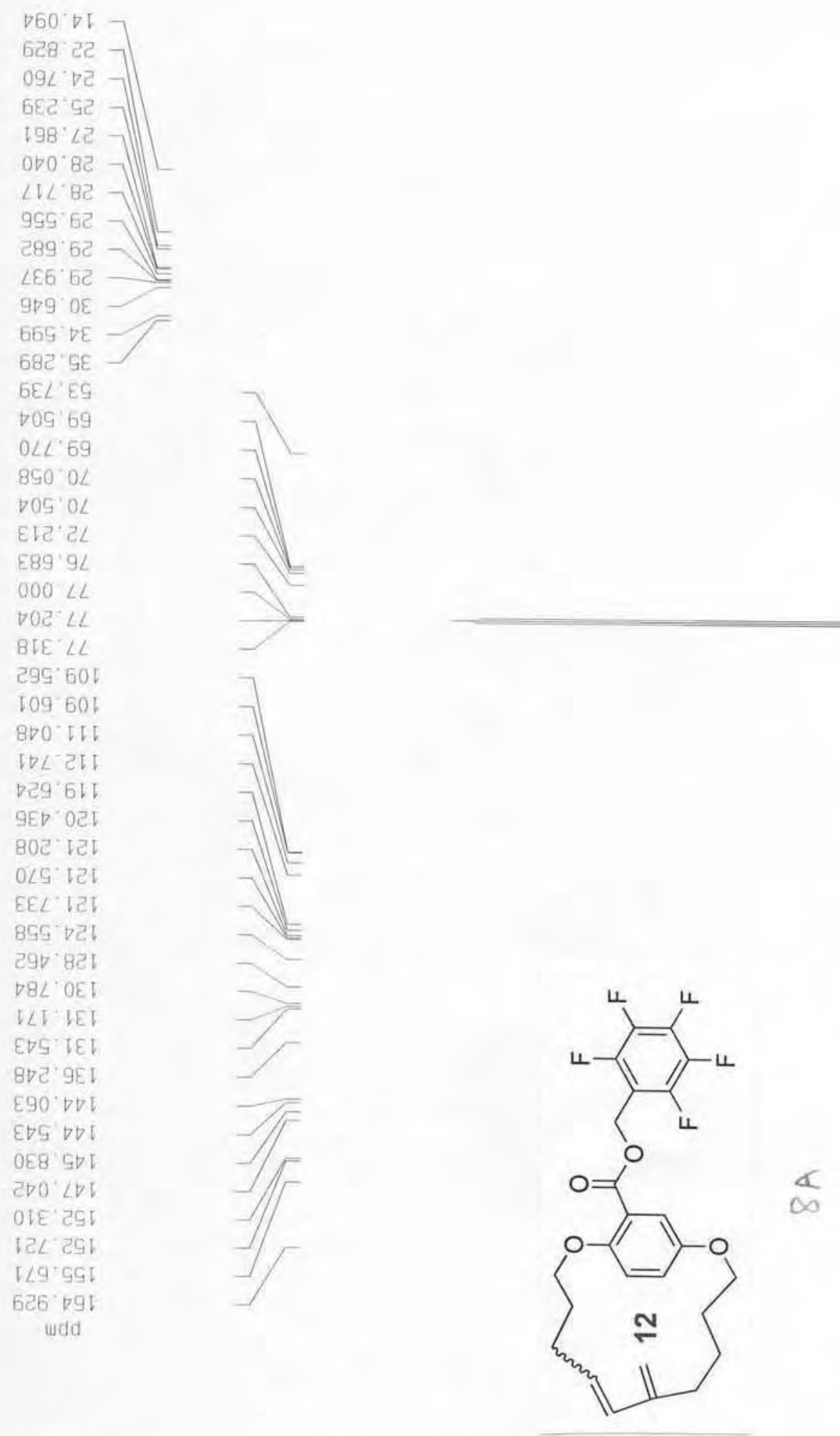


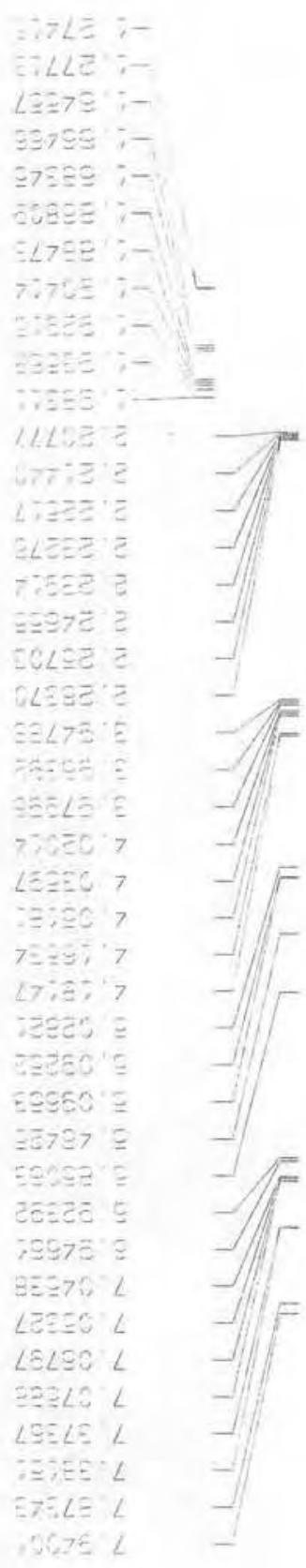

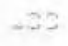
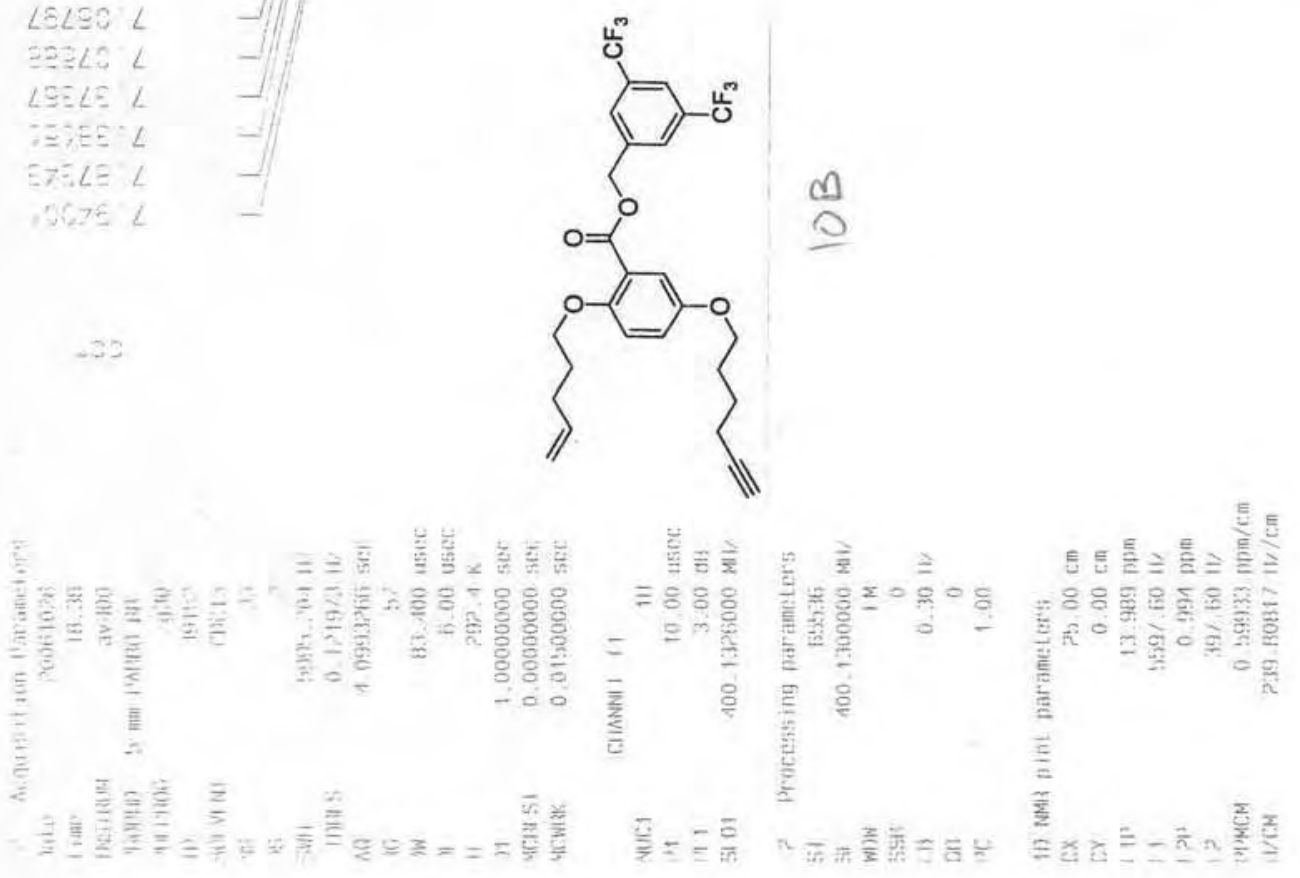


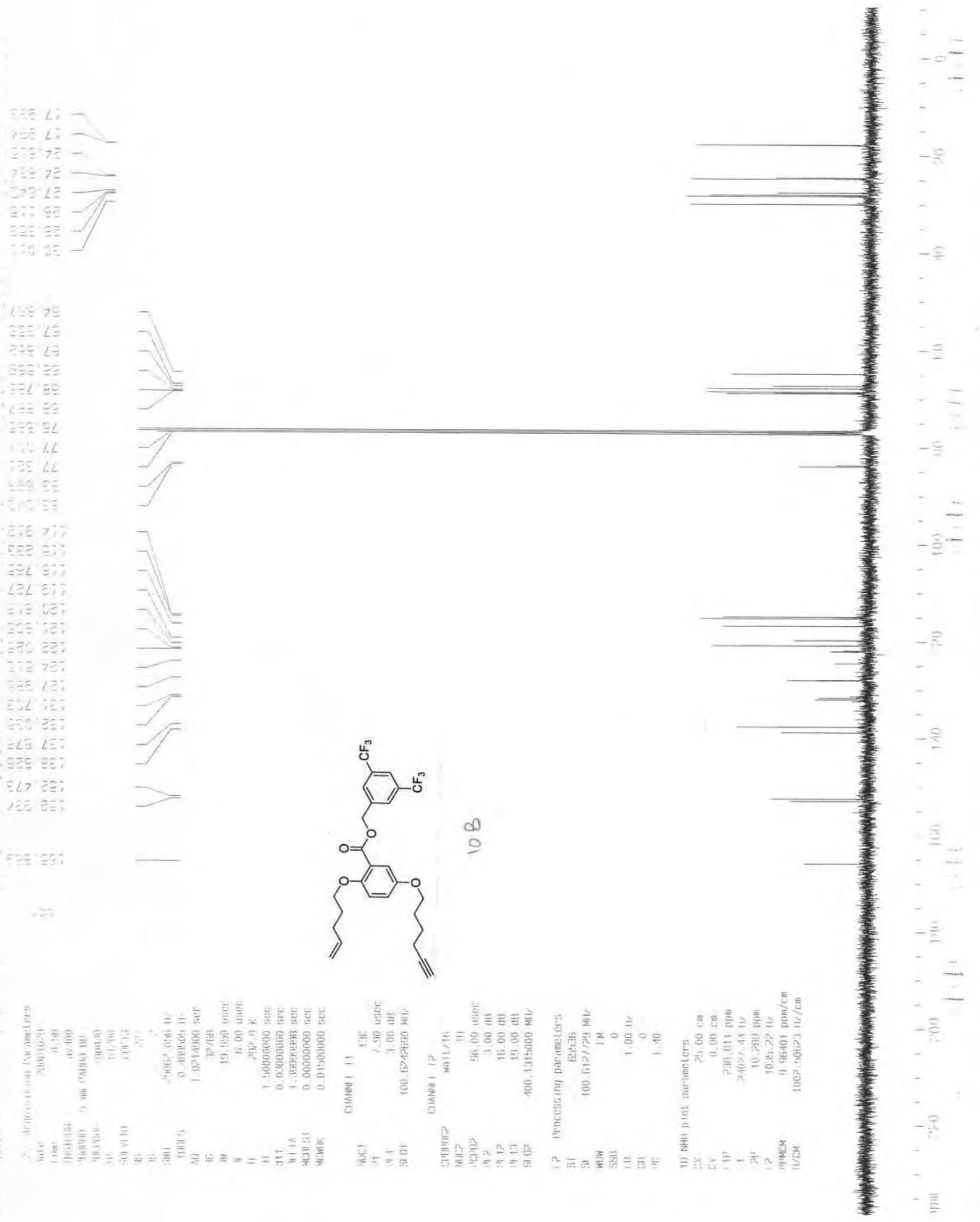




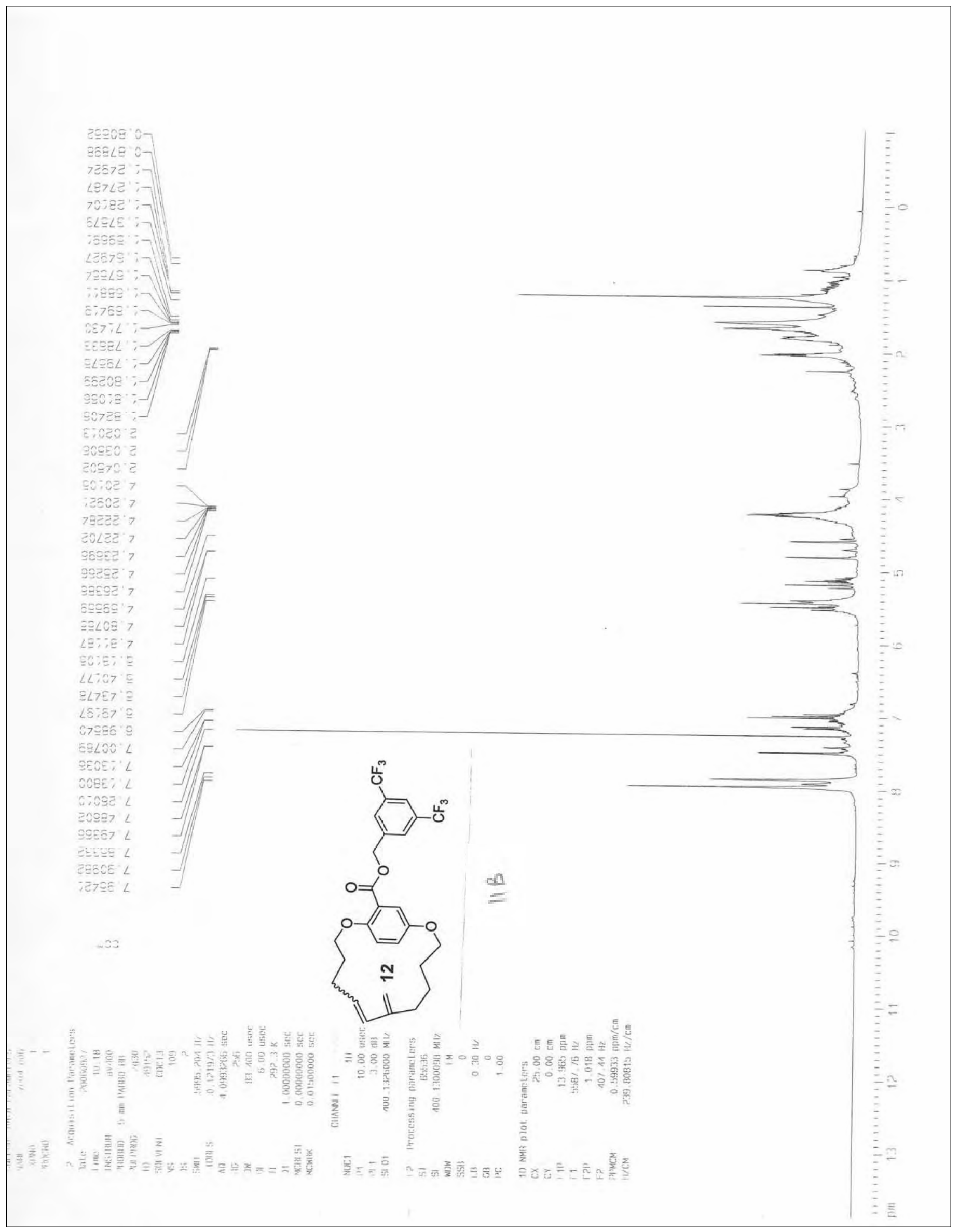




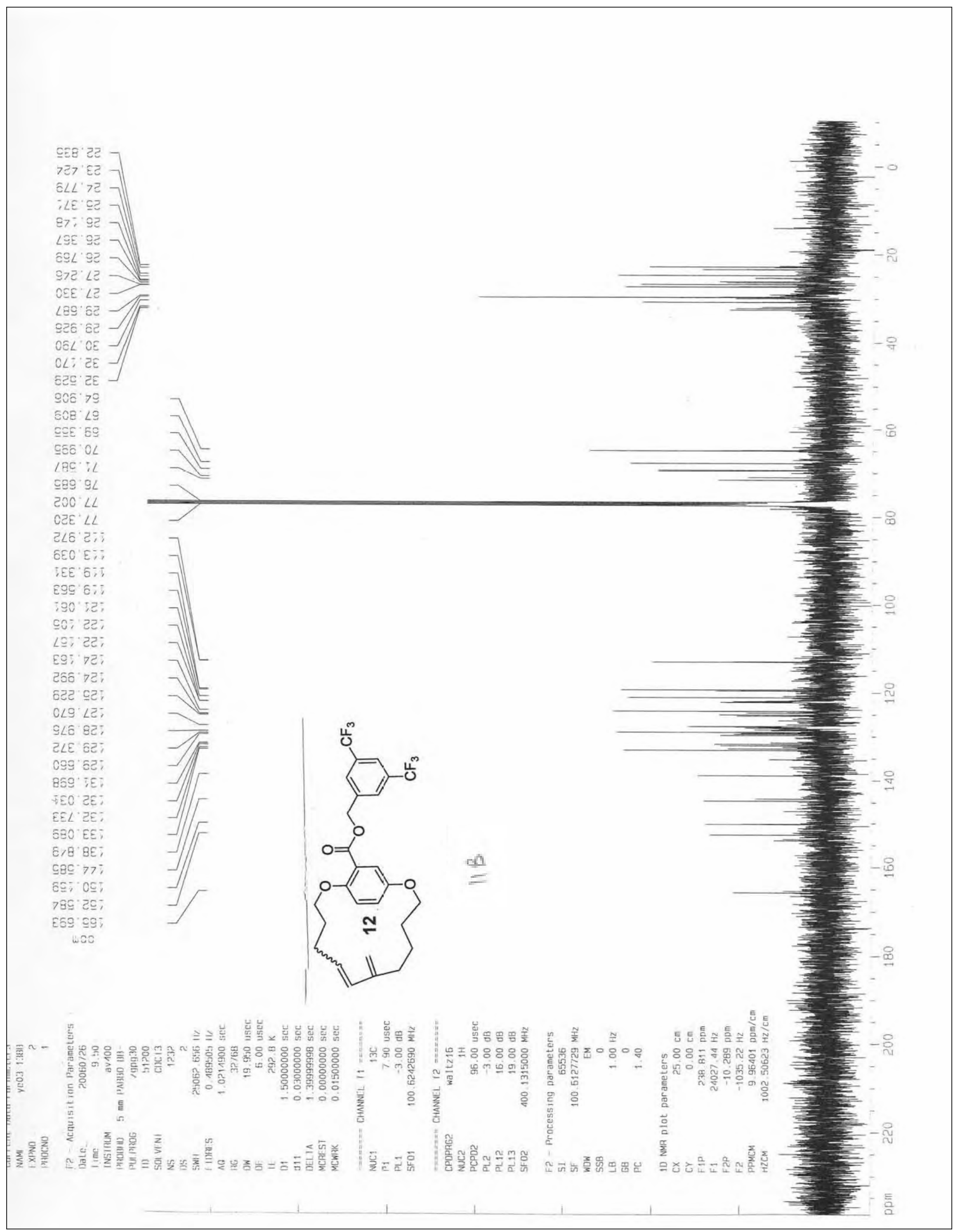



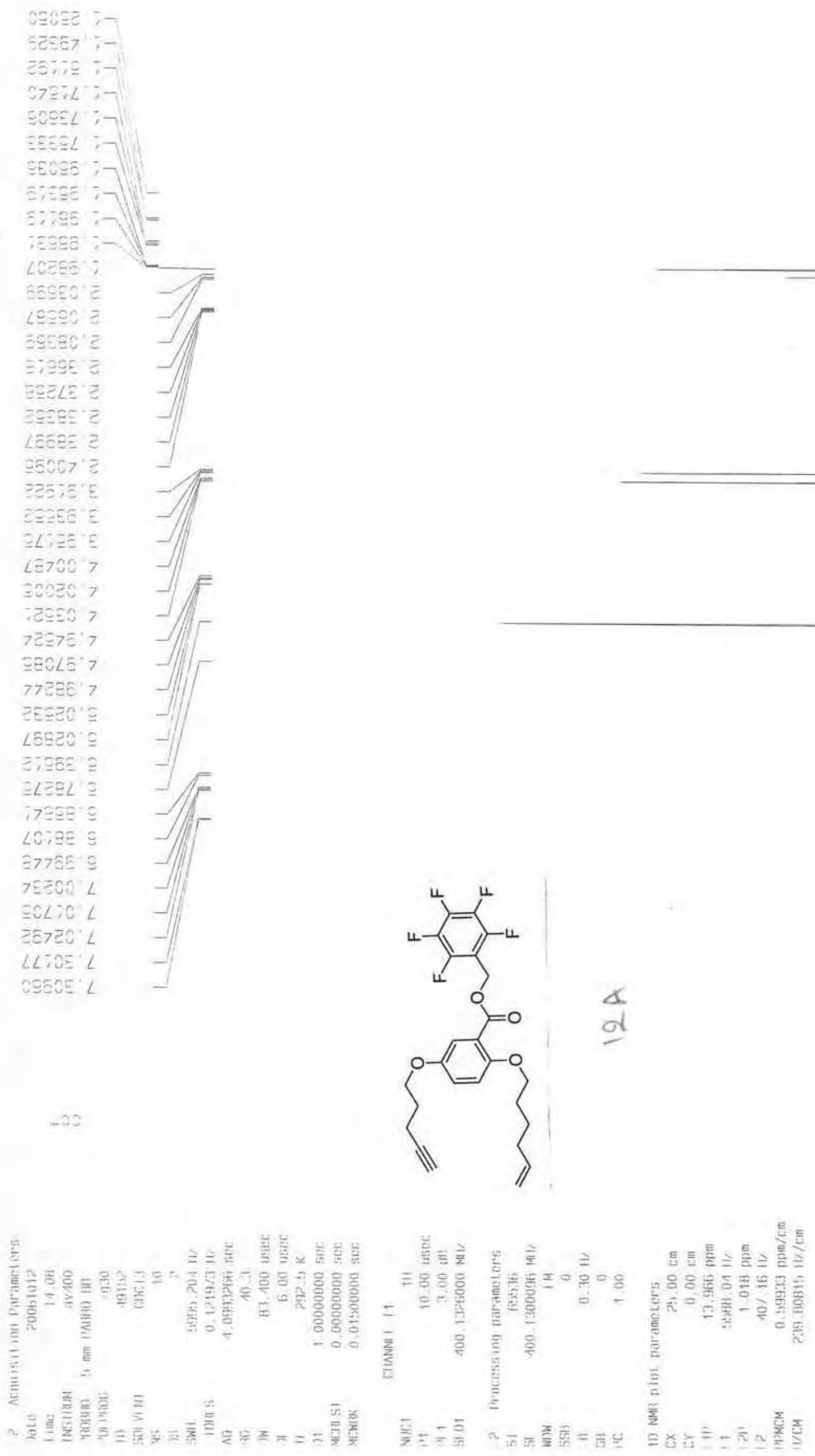


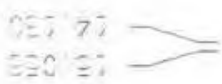

ELO $E Z$

हर० इ5

ESO हE

SE?

\section{LE}

$\Rightarrow 7$ 능

BSL $g 9$

ELE' $E S$

967 59

อ2ड $\cong L$

एOC $\angle L$

E:E' $L L$

उडs हe

\section{LEE $7: \%$}

ㅈis $7:$

LOE $B:$ :

CLE 6 :

25: 03

ELS ES:

67: ह5:

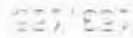

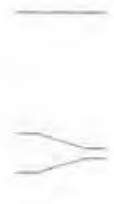

स:-

$+20$

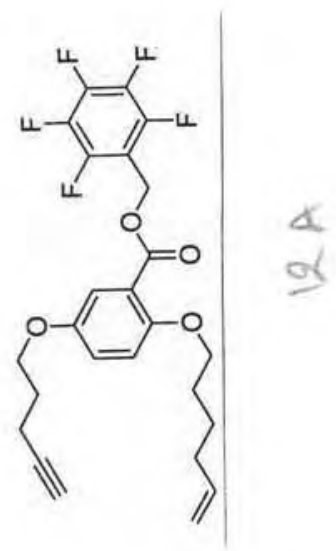

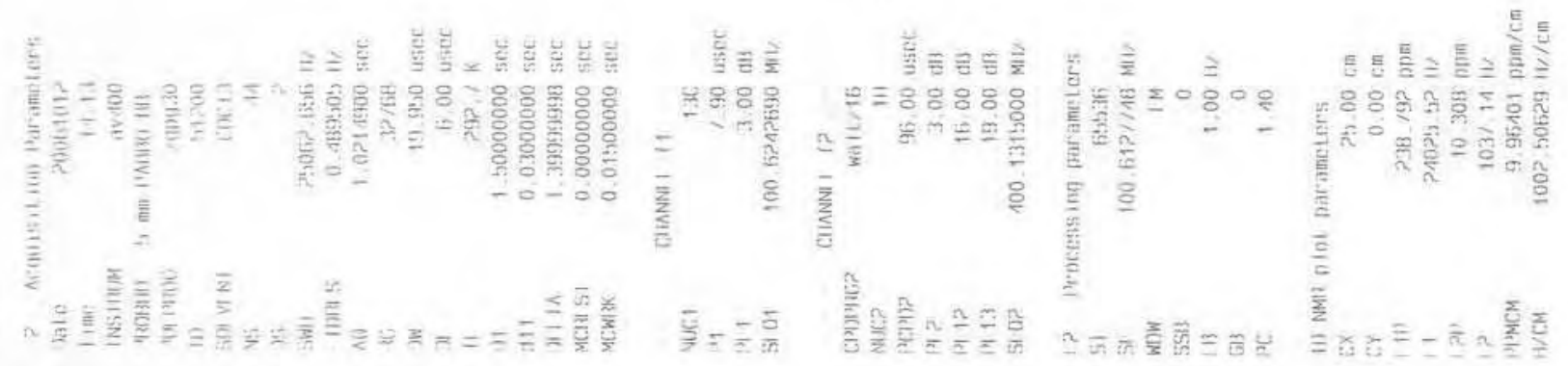
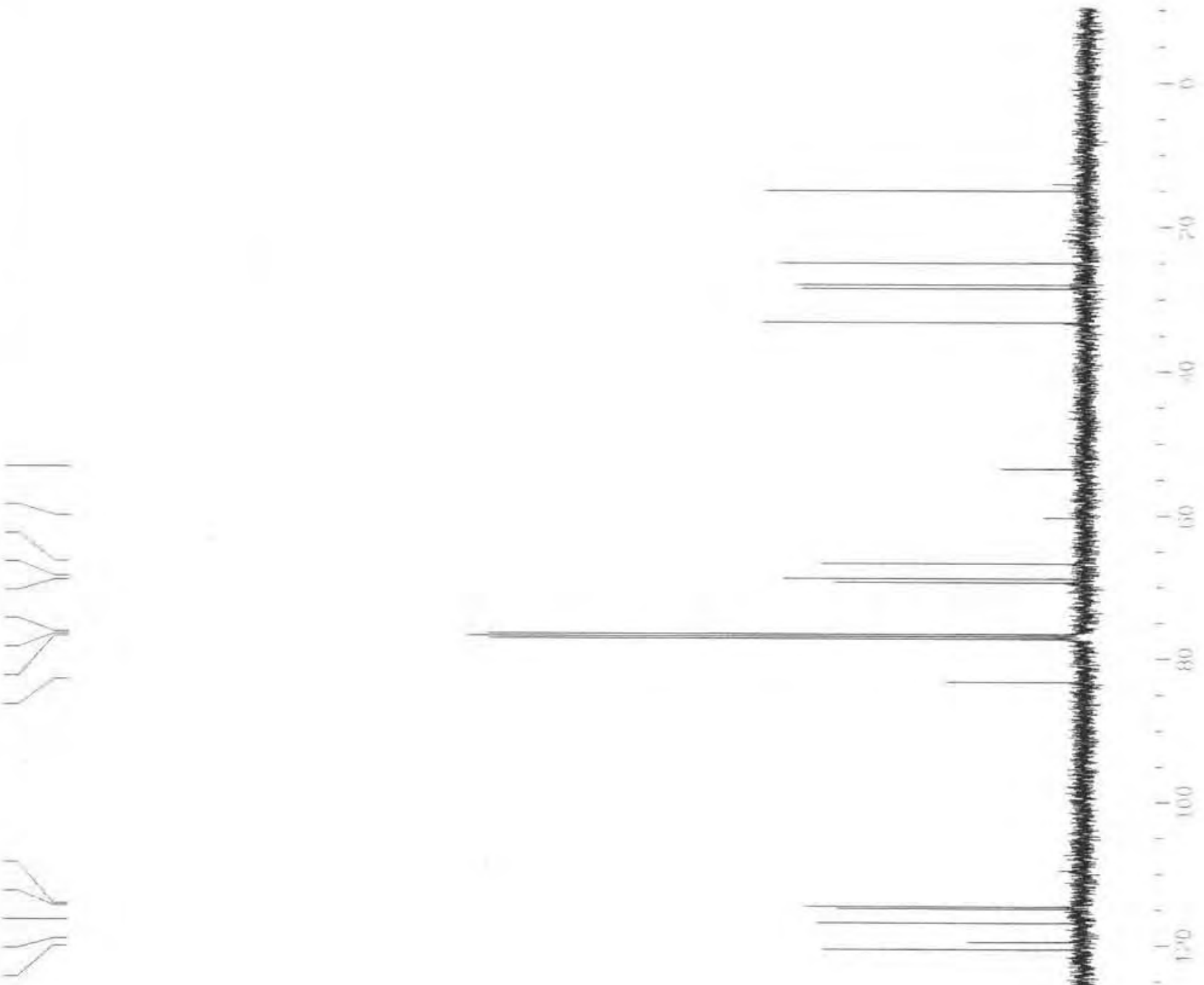


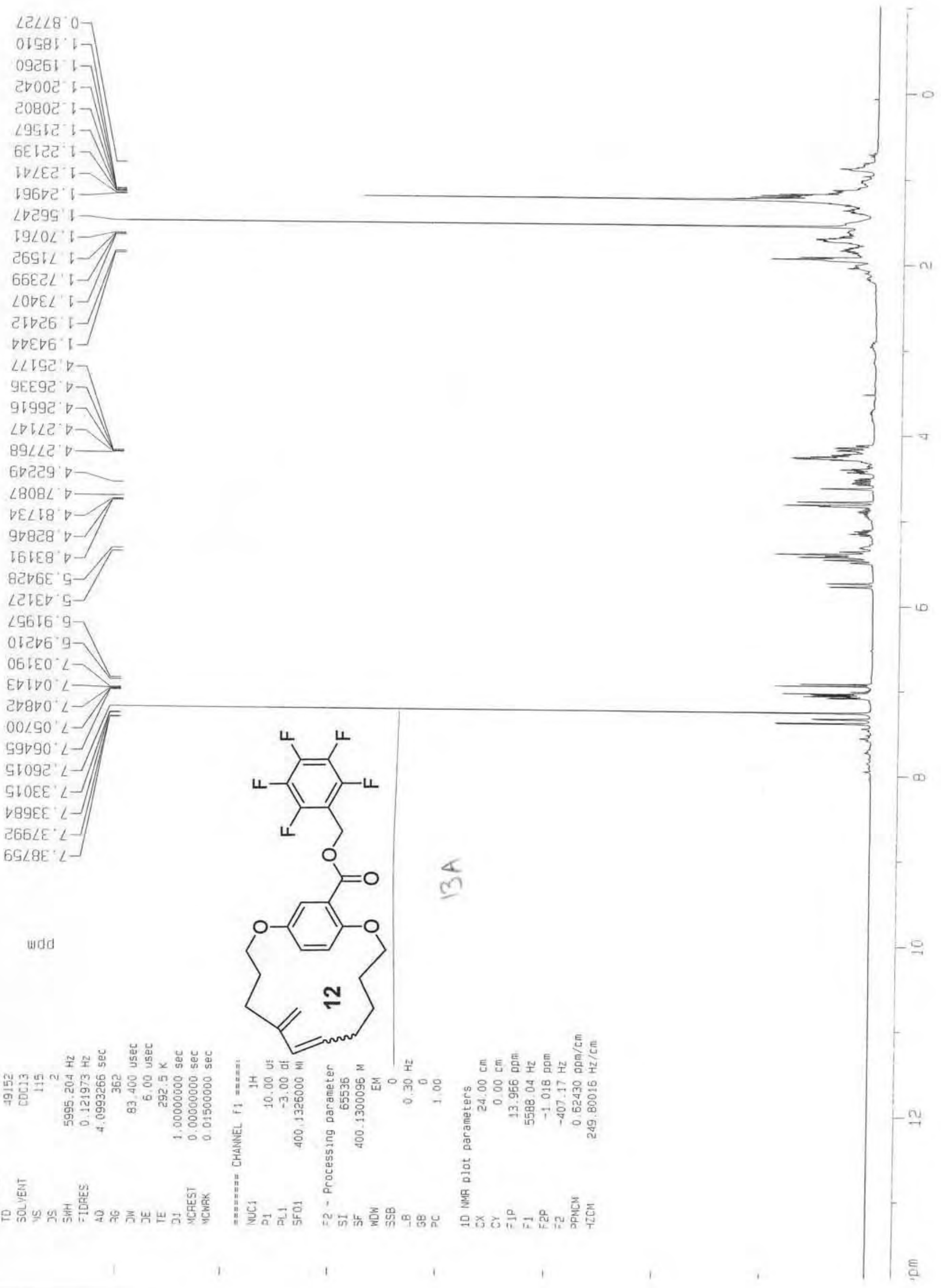



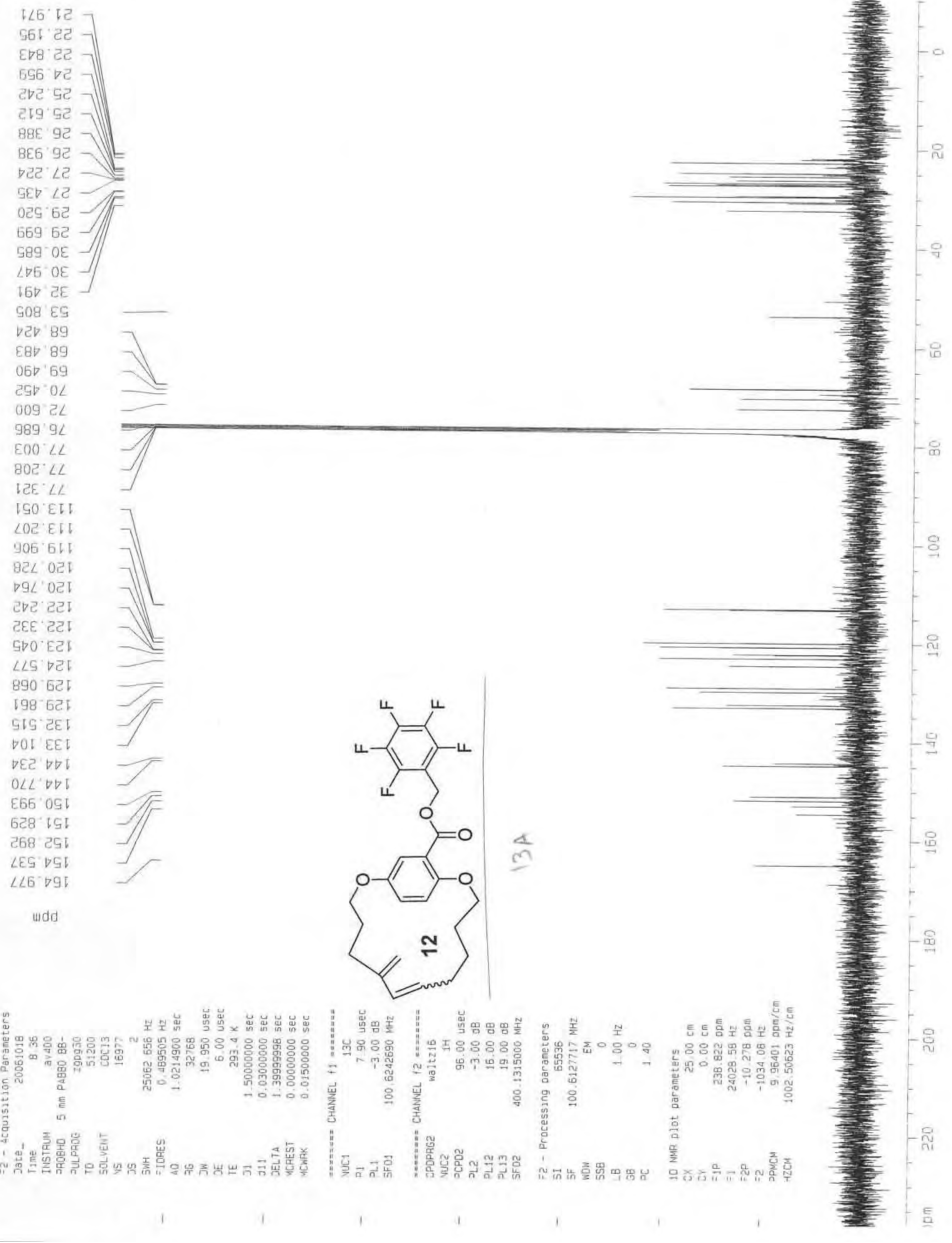

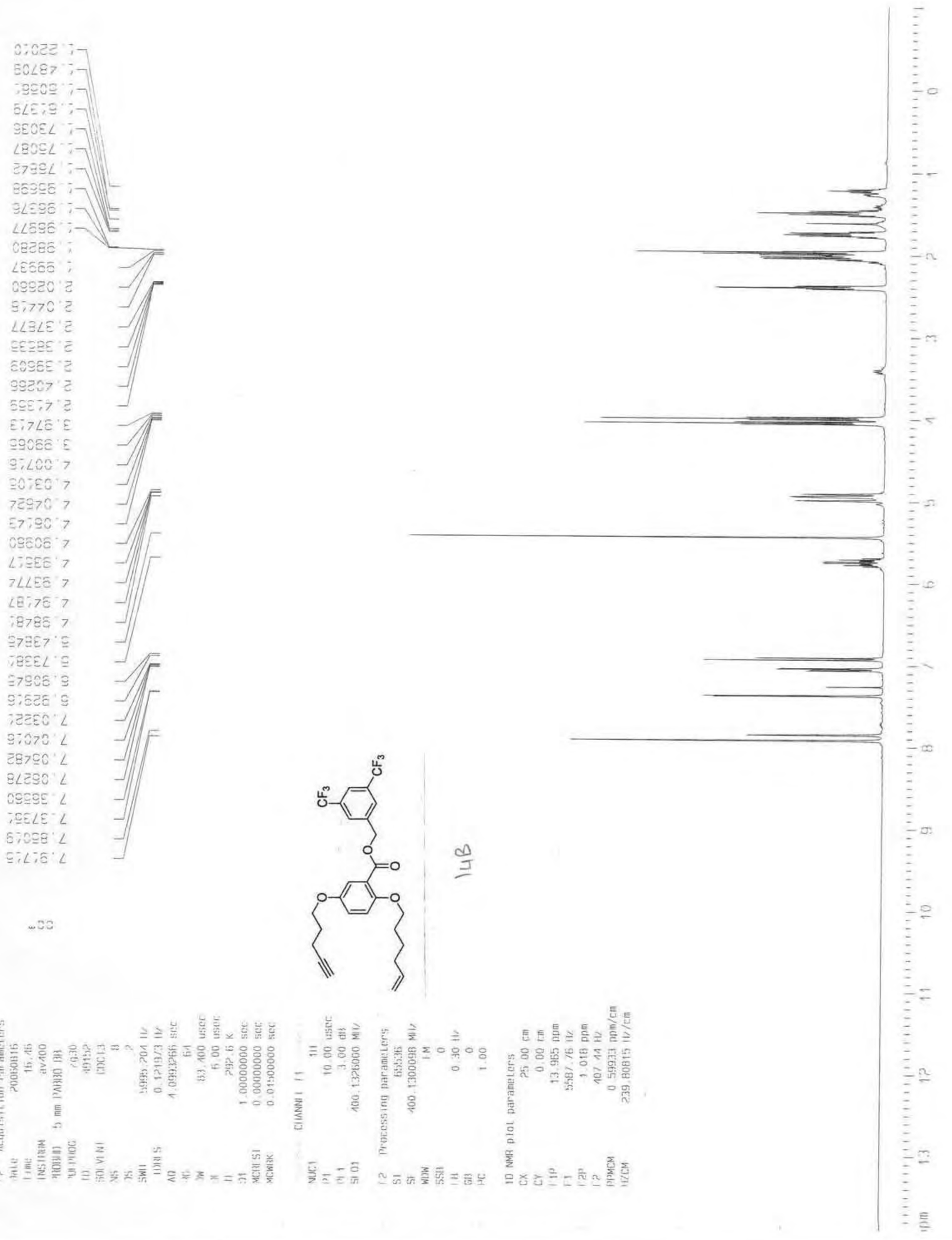
$9 \angle 0^{\circ} \mathrm{Gl}$

$\angle 90^{\circ} \mathrm{SC}$

$\angle 80^{\circ} 82$

oIS 82

$86 t=\varepsilon$

$568 \vee \nabla 9$

$662 \cdot 99$

ป 16.89

$\checkmark 8 E^{\circ} 69$

โ89. 92

$666^{\circ} 9 L$

ดเE $\angle L$

$90 \varepsilon^{\prime} \varepsilon 8$
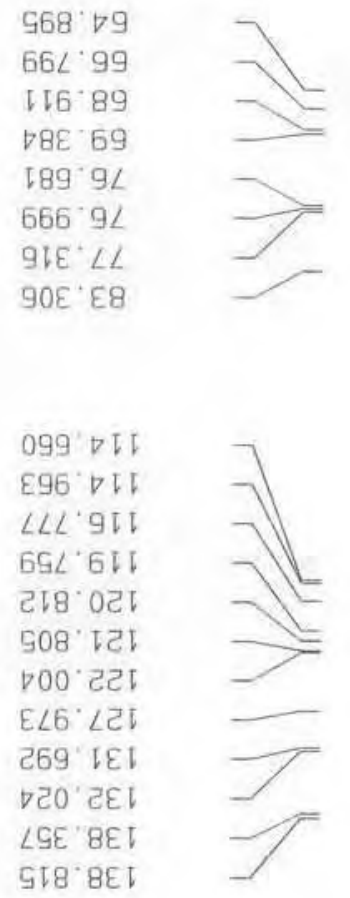

จЕट' टढा

LEV EST

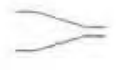

$6 \nabla 6.991$

wdd
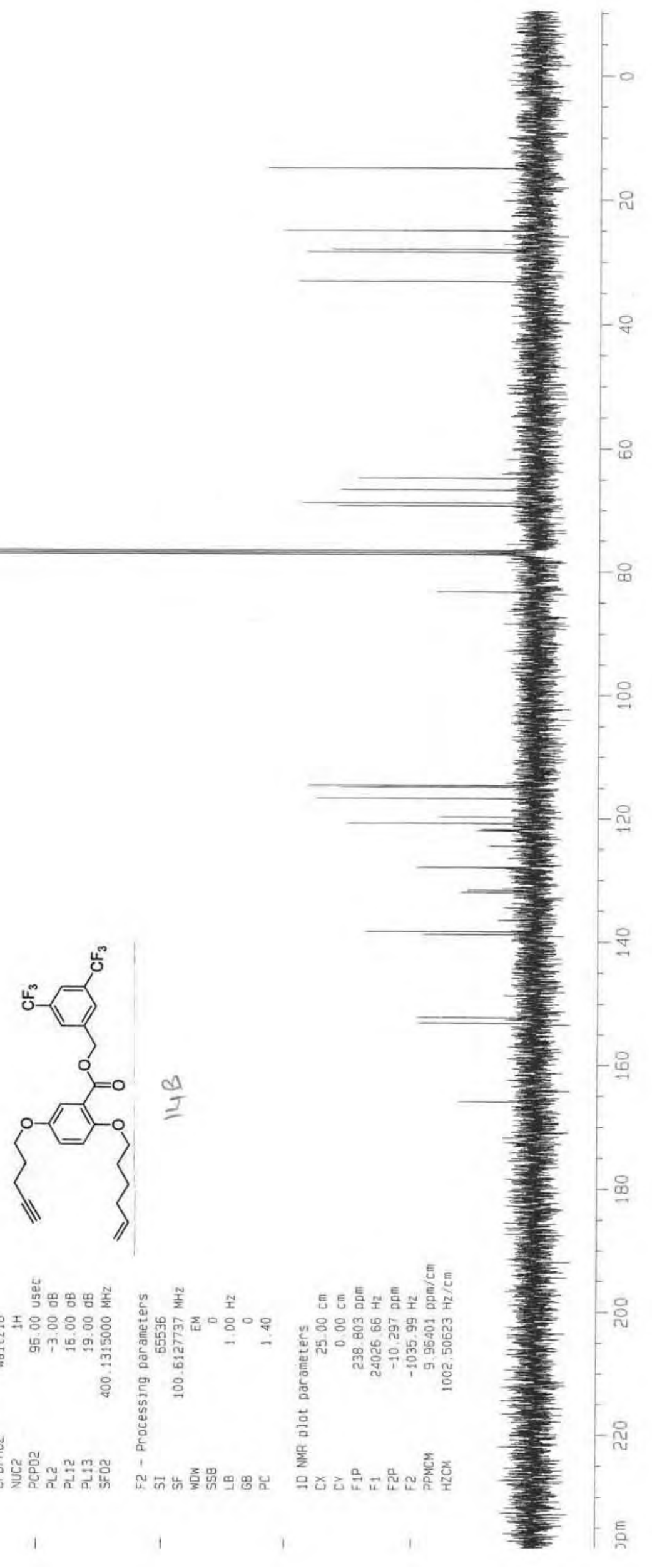

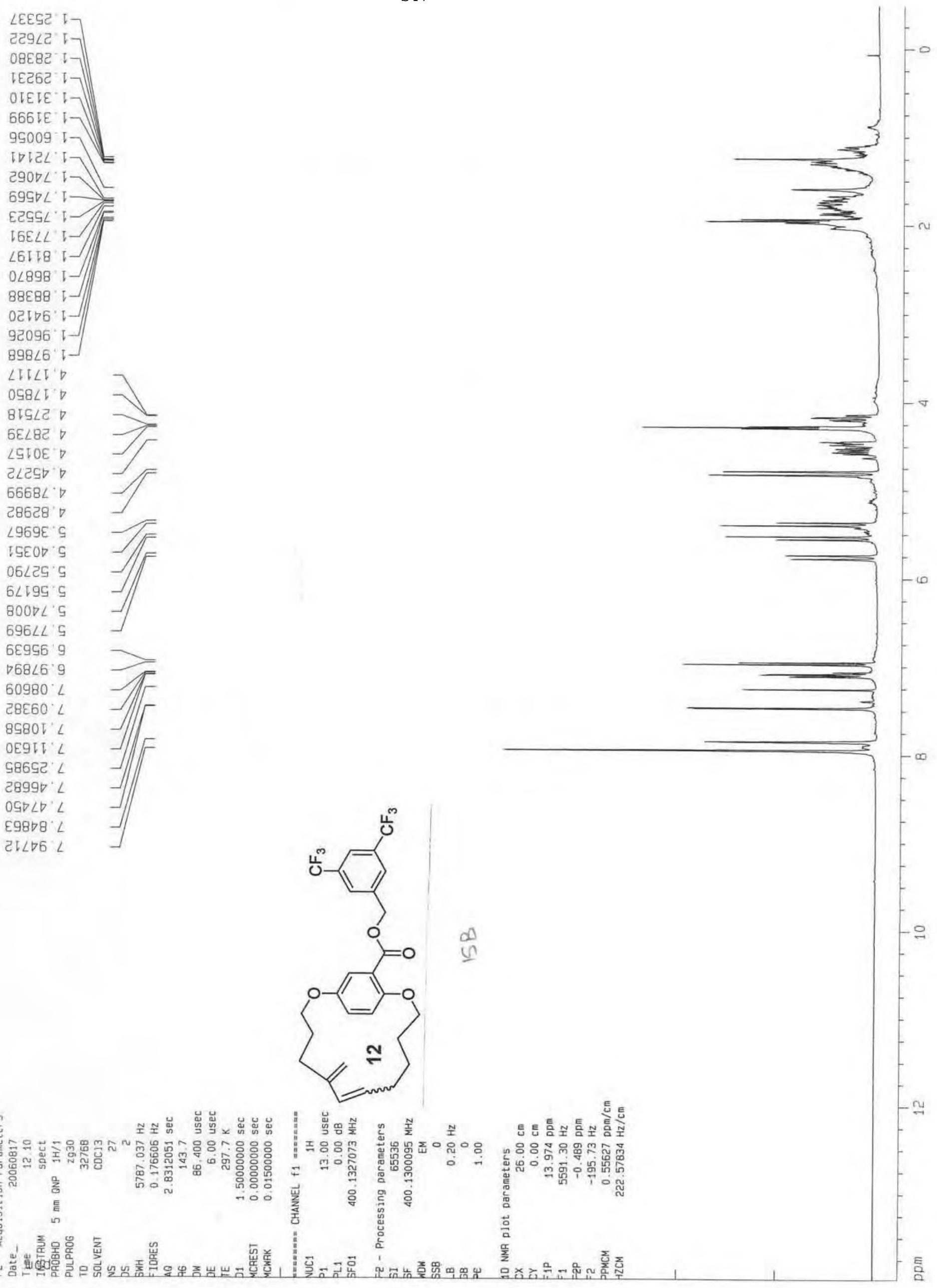


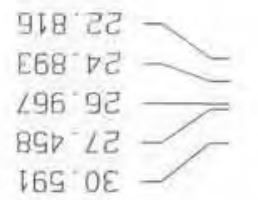

$\left\lceil\angle B^{\circ} \nabla 9\right.$

$90 \varepsilon^{\prime} 89$

टG० 89

ट89 92

$666^{\circ} \cdot 9 L$

$\angle E^{\circ} \angle L$
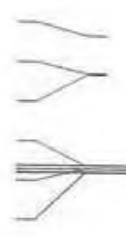

SOIEIT

$0 V L=6 L$

$998^{\circ} 0$ ट

E娒圮।

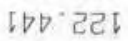

891 हट I

$\angle O L \angle C$ I

$868^{\circ} 82$.

$06 t^{\circ} \varepsilon E \downarrow$

6ट5 $8 \varepsilon \downarrow$

$S \nabla L^{\circ} \nabla \nabla \digamma$

898 0 51

เट6. I5

689 991

wdd
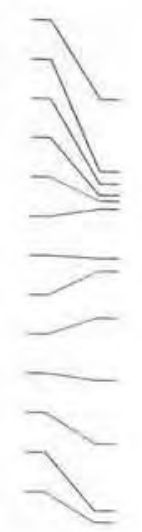

in
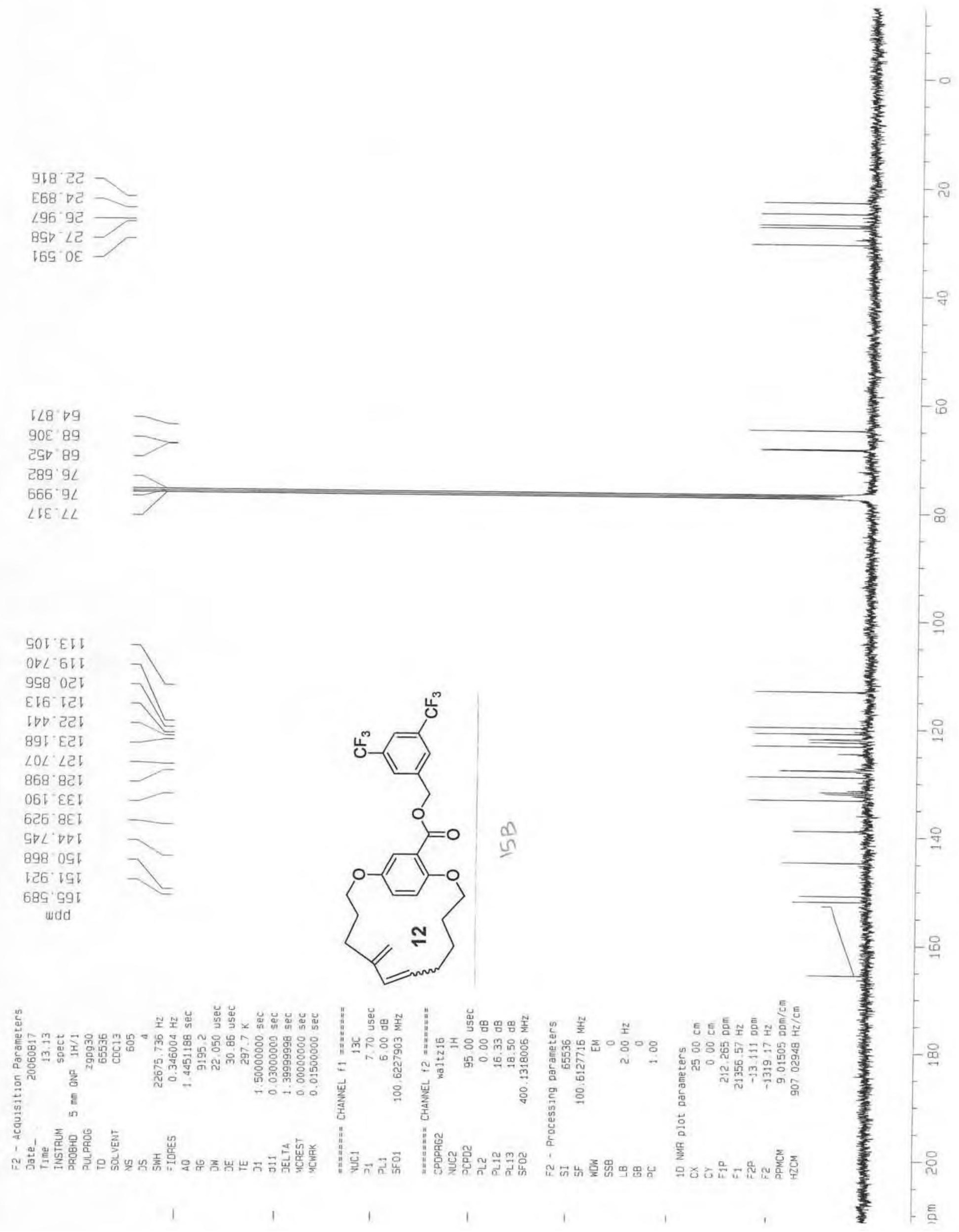


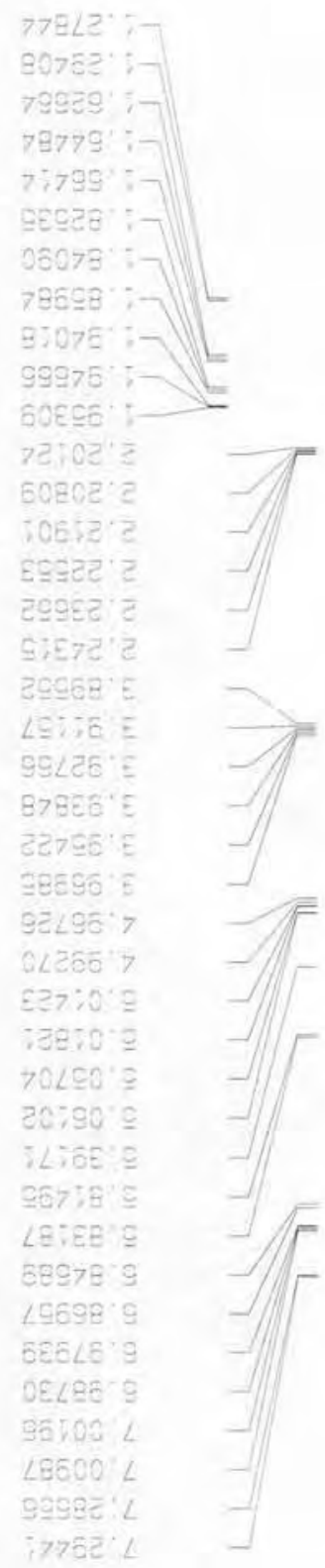

403
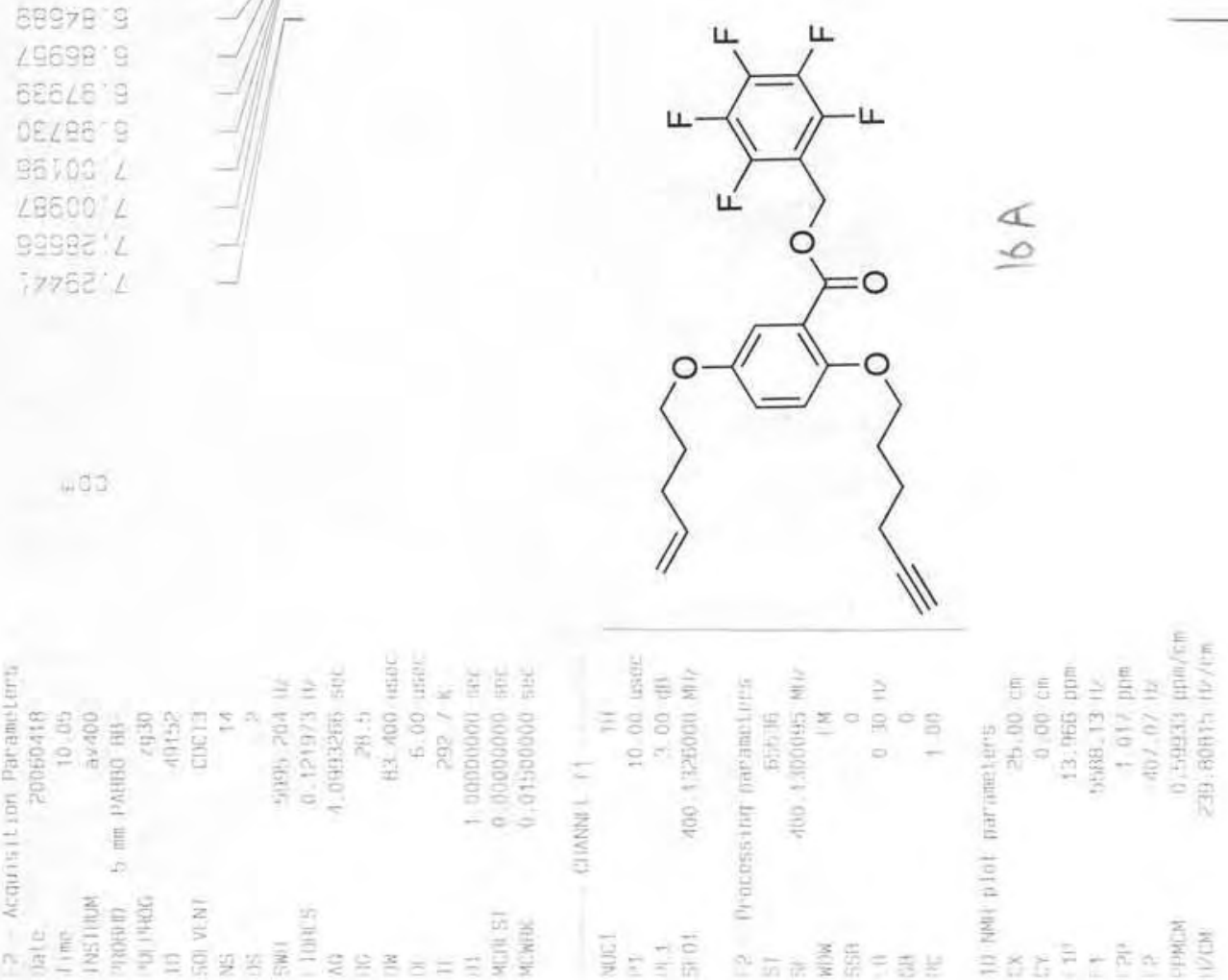

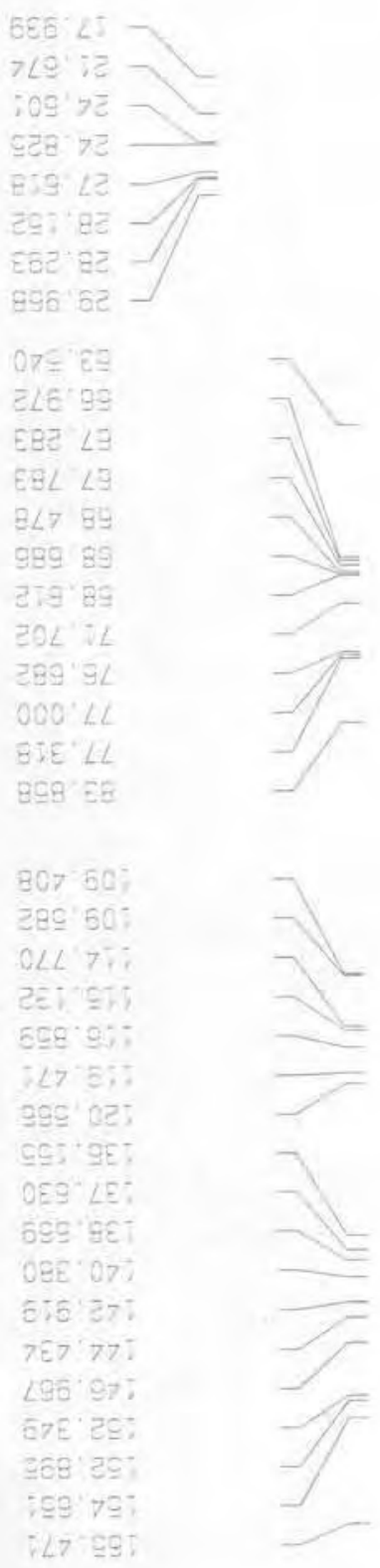

wes
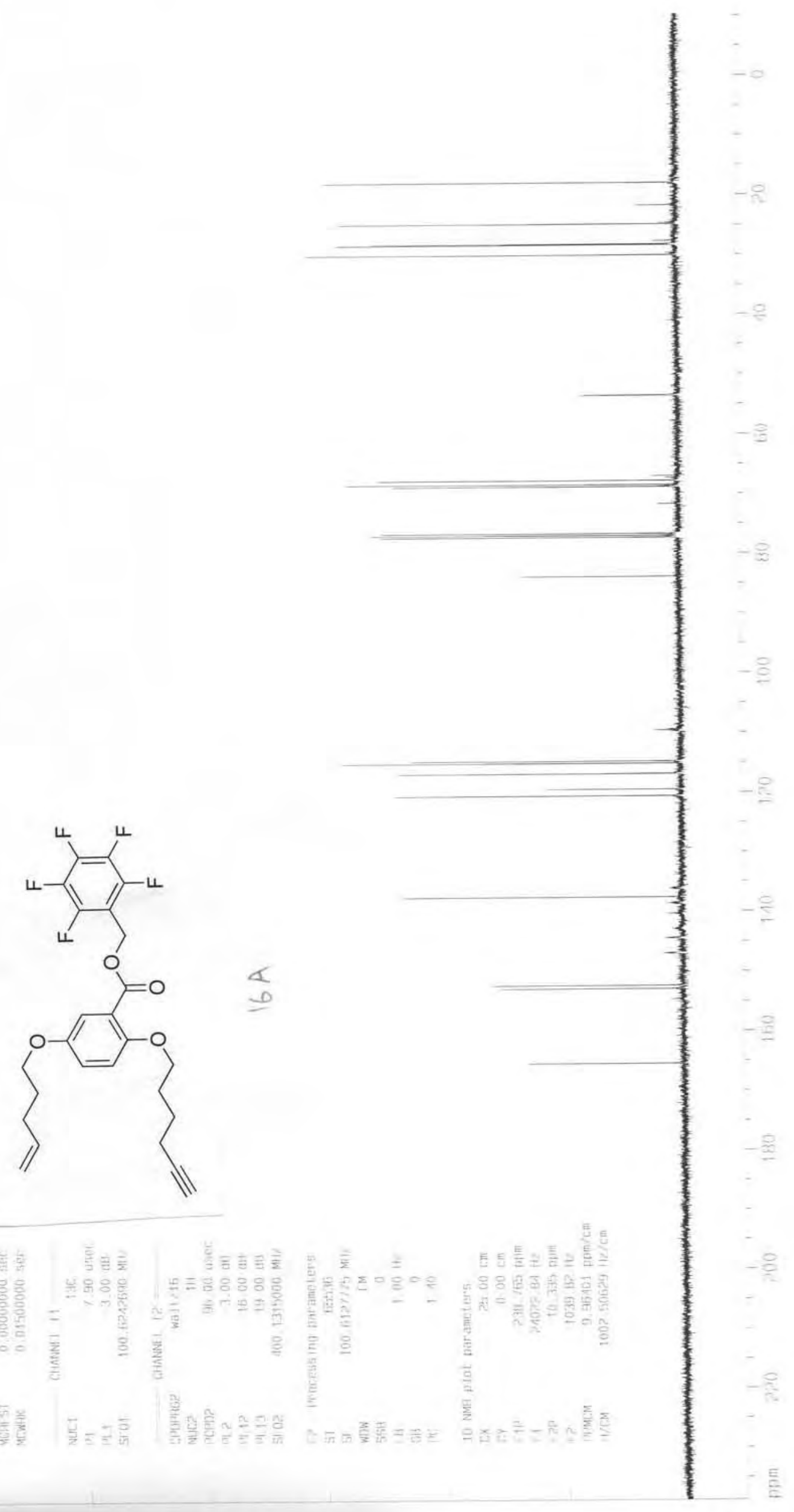


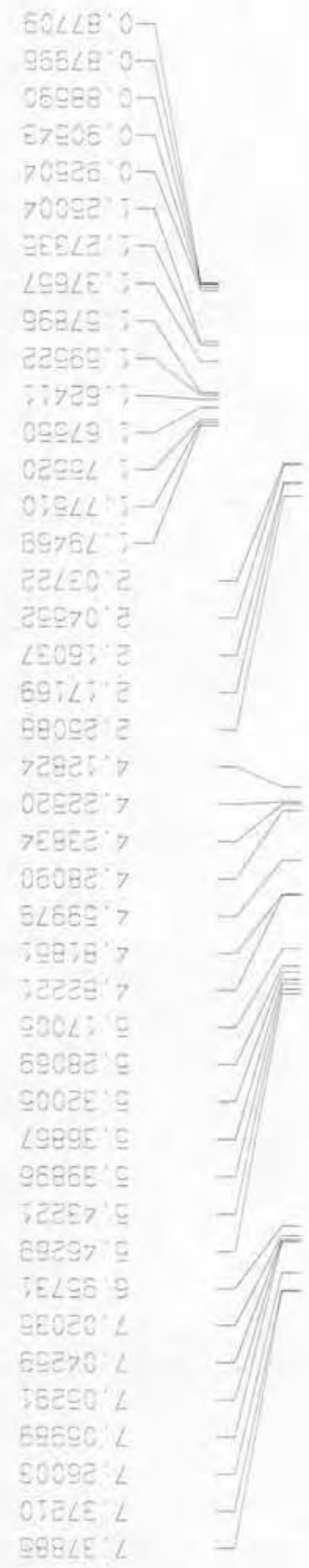

400

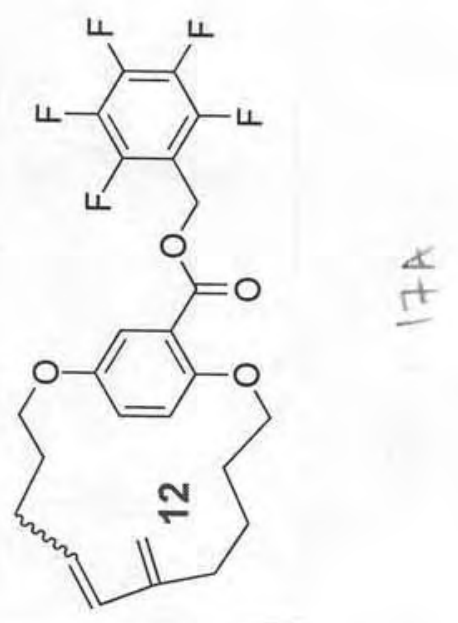

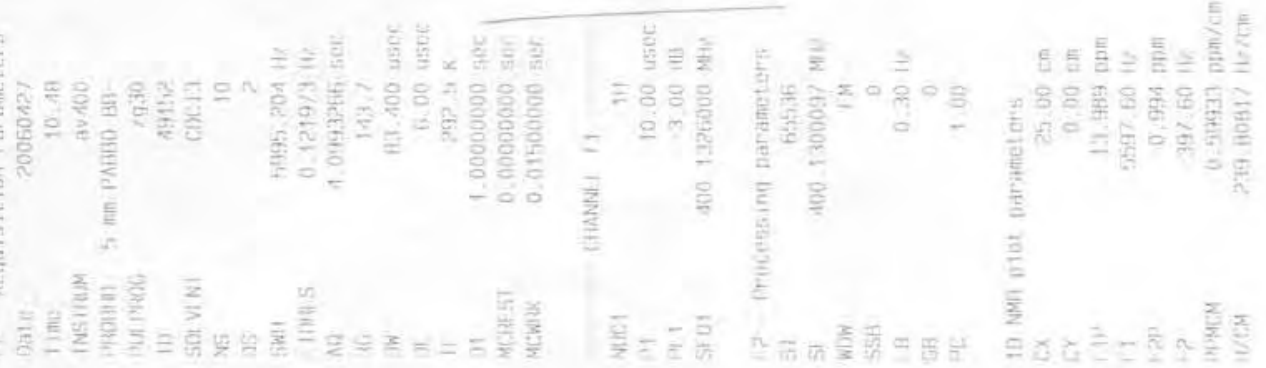


S52

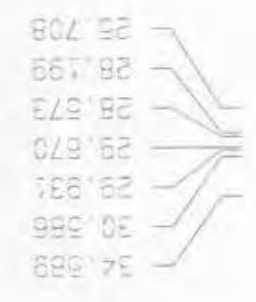

$\angle 98 \cdot 95$

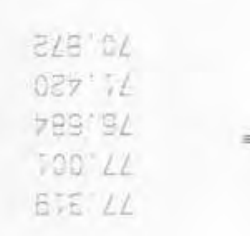

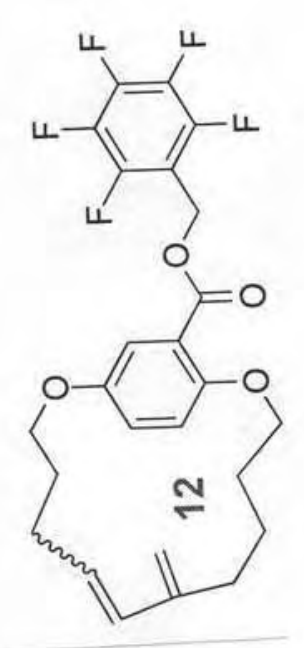

I

$-\frac{5}{2}$

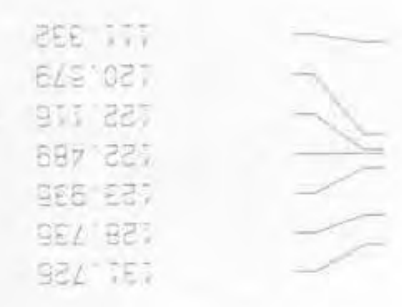

E99 57:

EET EE:

800 Te:

แม2

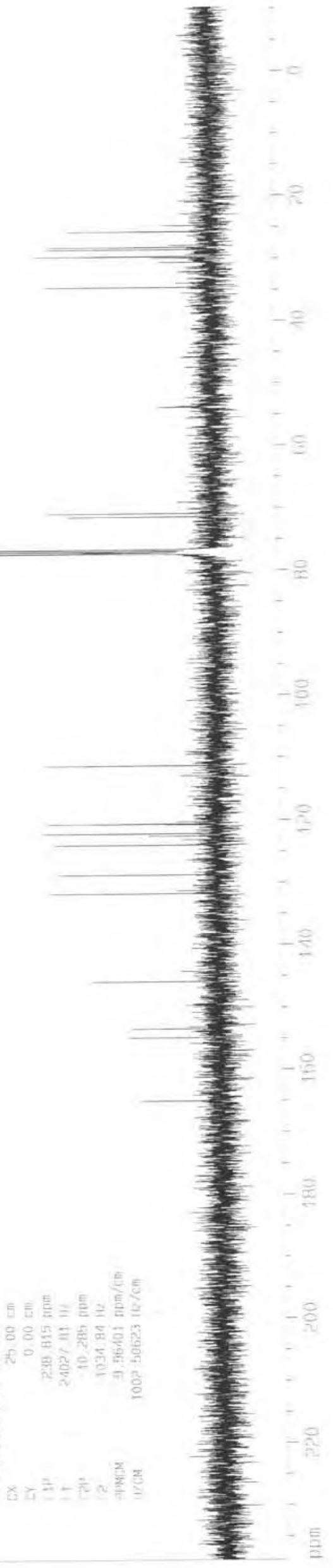




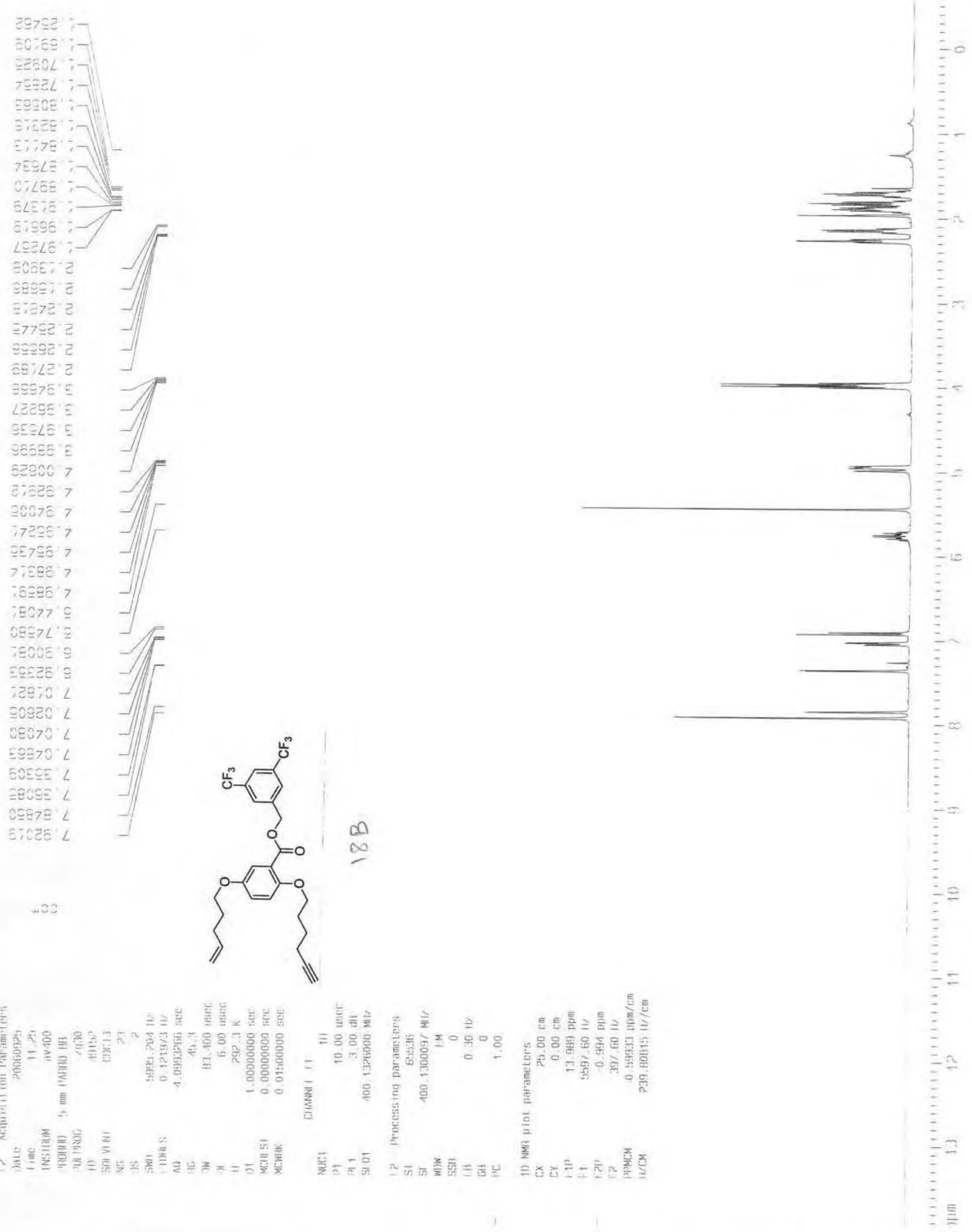


$2: 80$ :

ธटล 73

Qร:'

LEe हอ

\section{5沱 79}

$375 \angle 9$

¿ㅇ 99

$\exists=L$ ป

ige 91

$356 \Xi L$

QSE LL

¿LE EQ

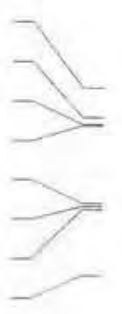

E00.

Eา: $2:$ :

ese

टEL E⿱一

टEL OE:

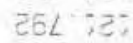

हEO टE:

EUE $7 Z$;

TडE $L E$ :

Ese 湜:

इट0 วह:

टड़ LE:

¿LL $9 E$ :

ESE $25 ;$

200 Ee:

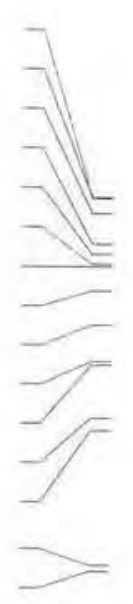

$756 \leqq 9 ;$

was

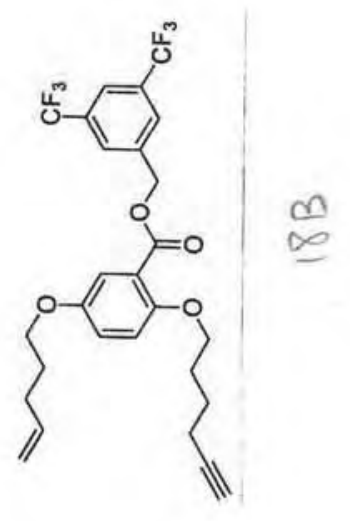

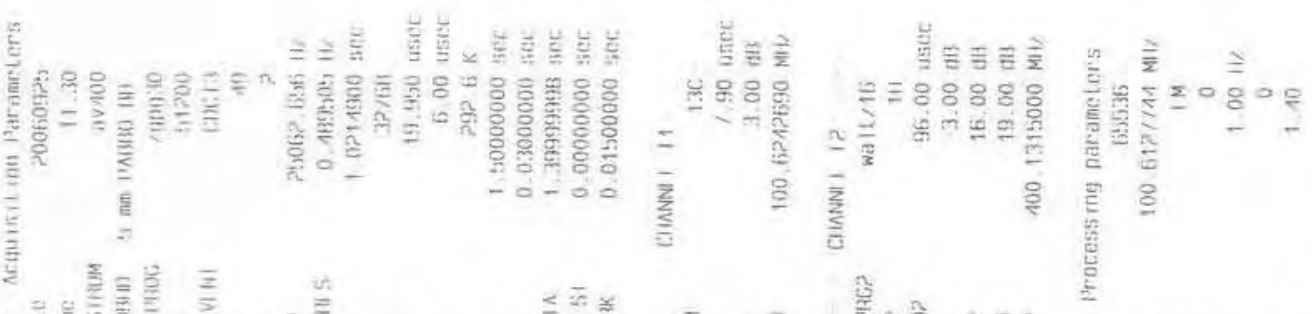

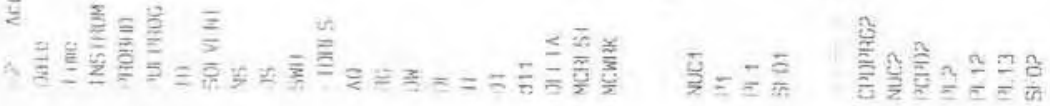

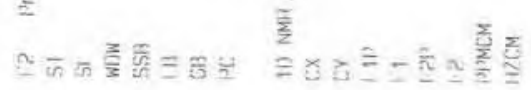

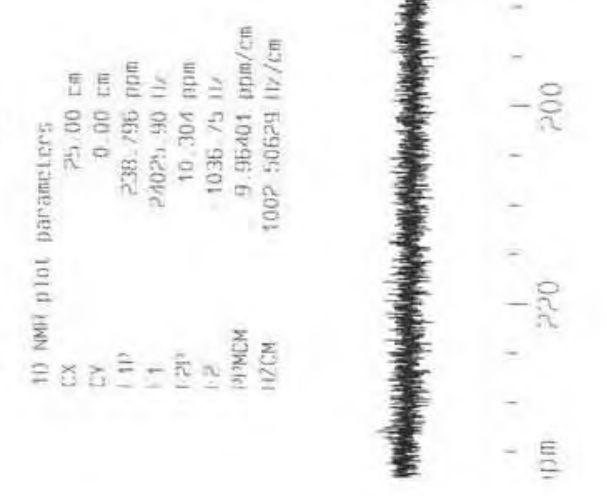




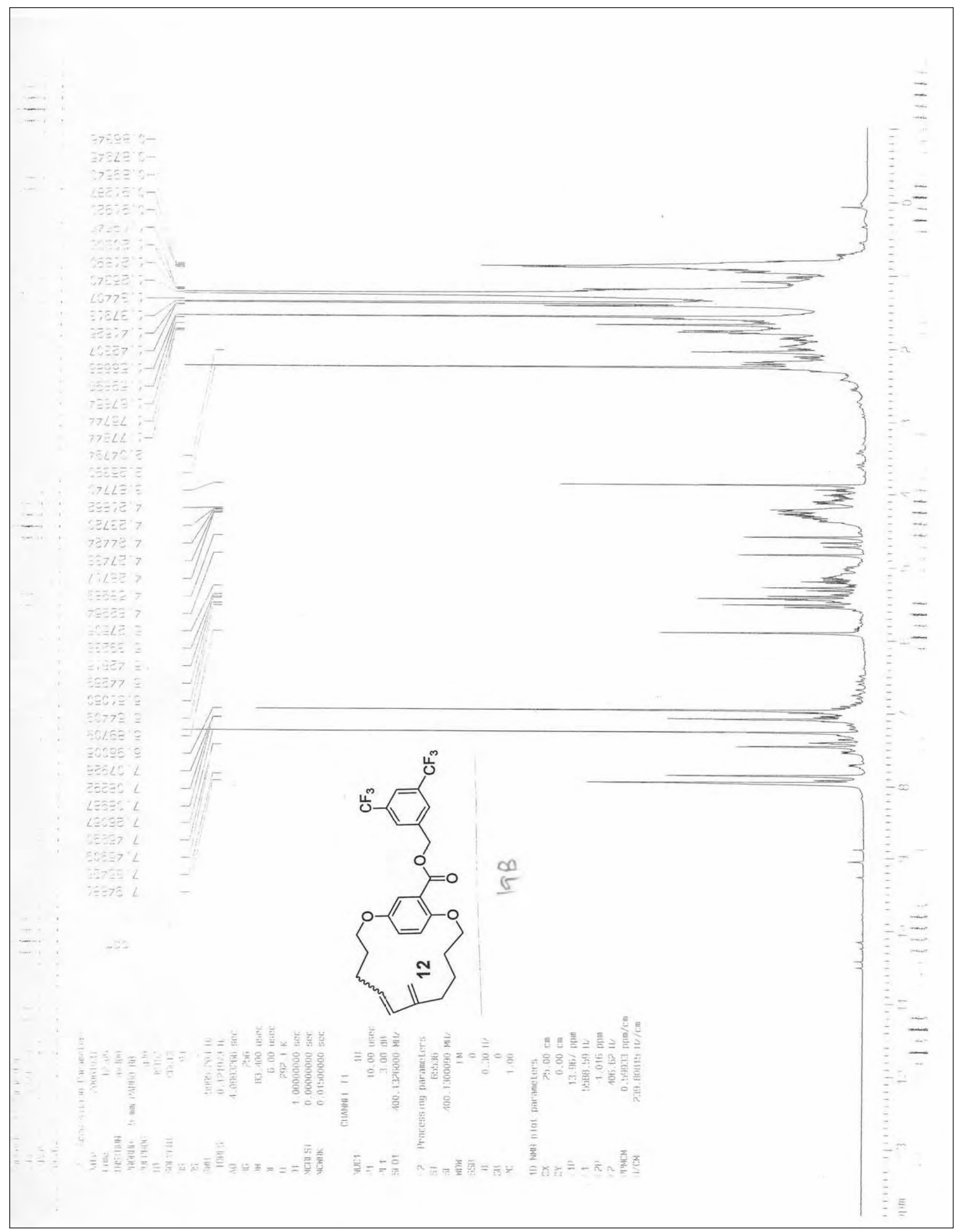


S56

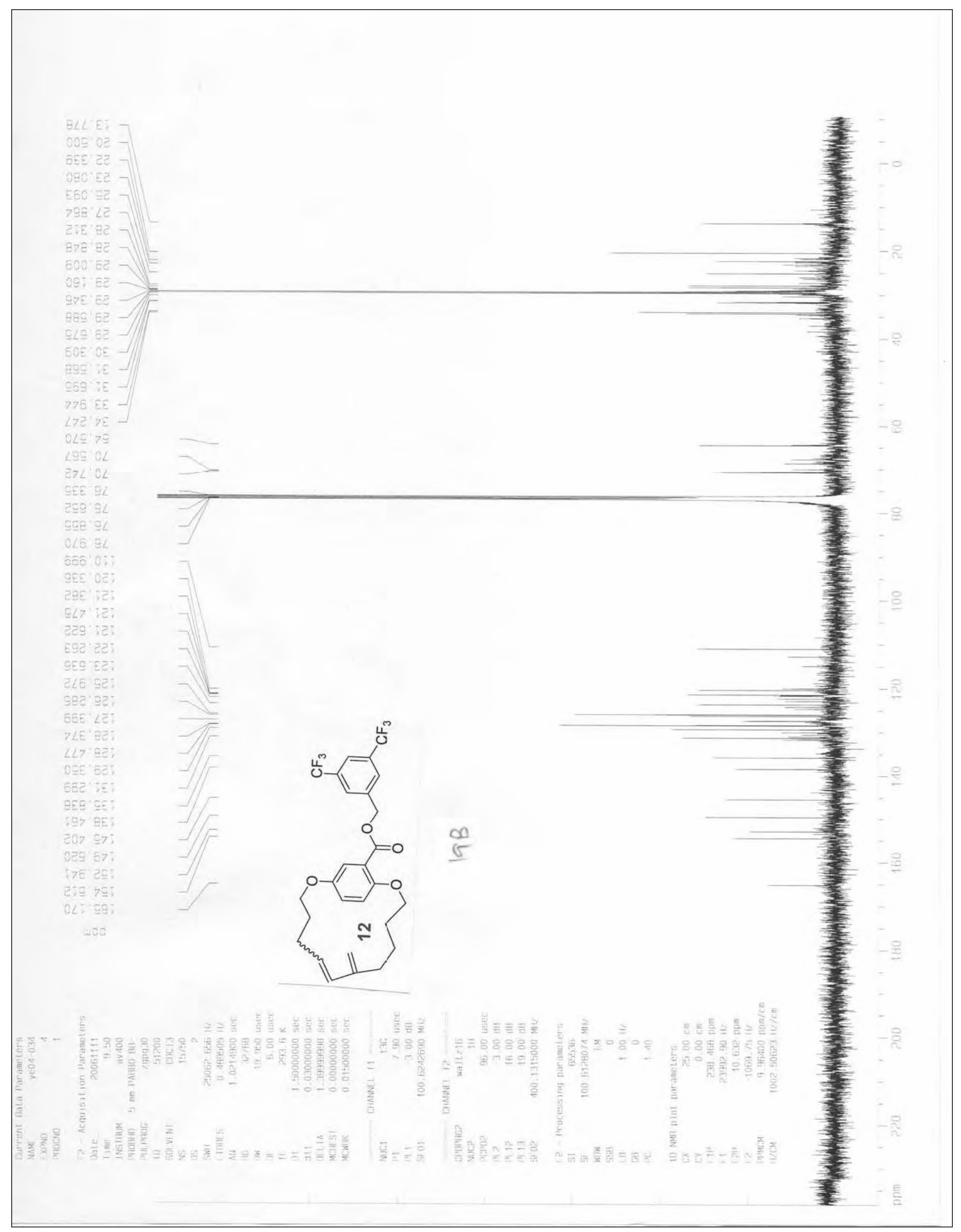



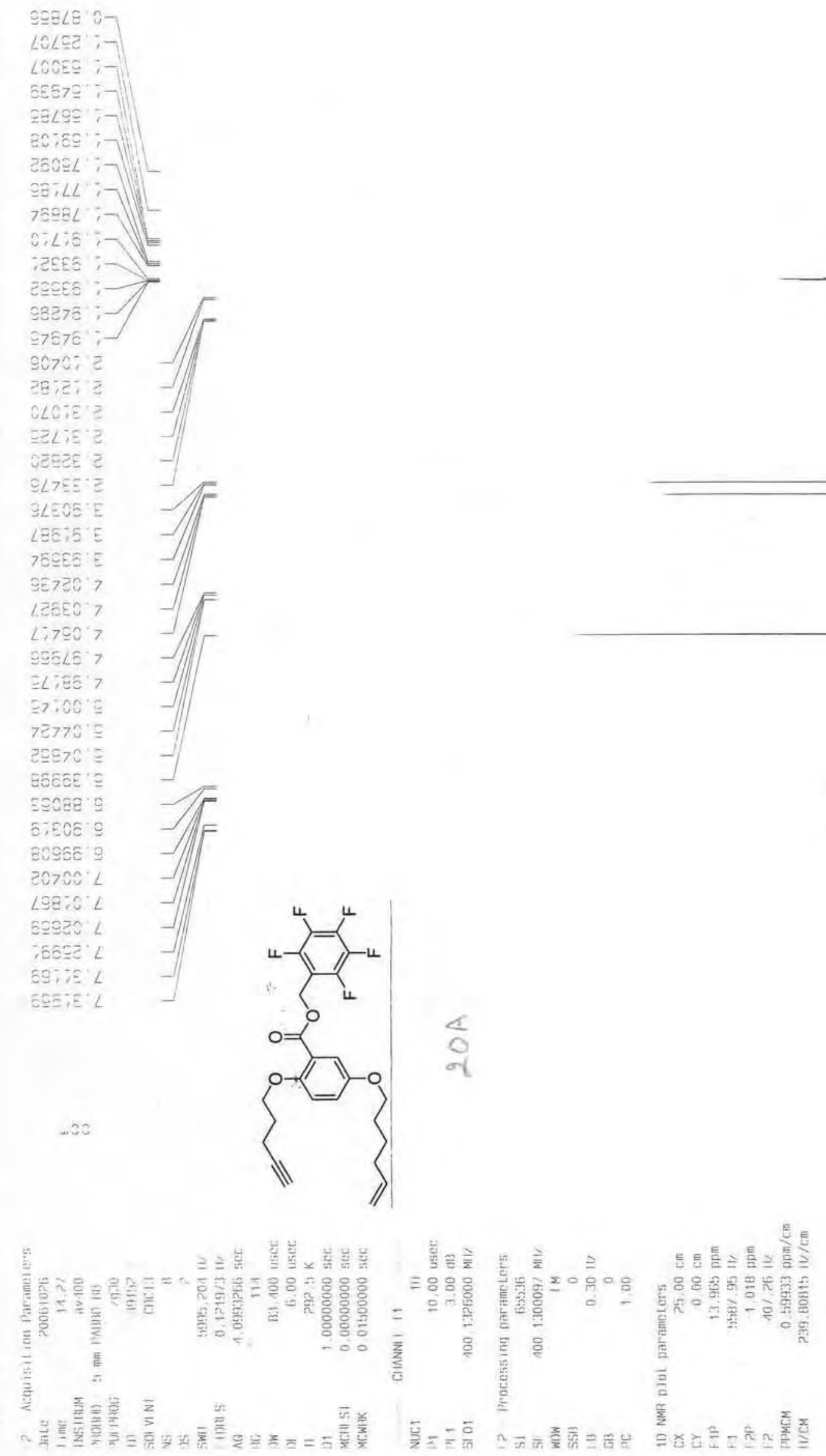


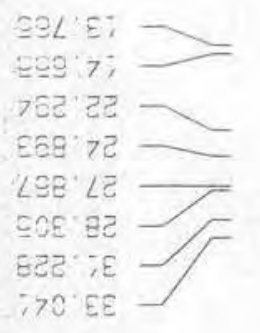

\section{วอट ฮฐ}

EEE $\angle E$

ट0, 89

ธะอ 89

OTE $O L$

LSG $G L$

¿LE $E L$

ELS ¿ะ

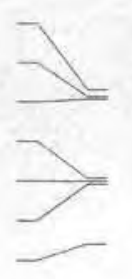

E:7 $7:$

ХEE 7 ,

ธระ $9:$

ह5, का?

ร88 23 ?

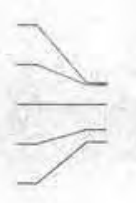

90:

श०5 5:

CED 32:

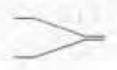

LL:อะ:
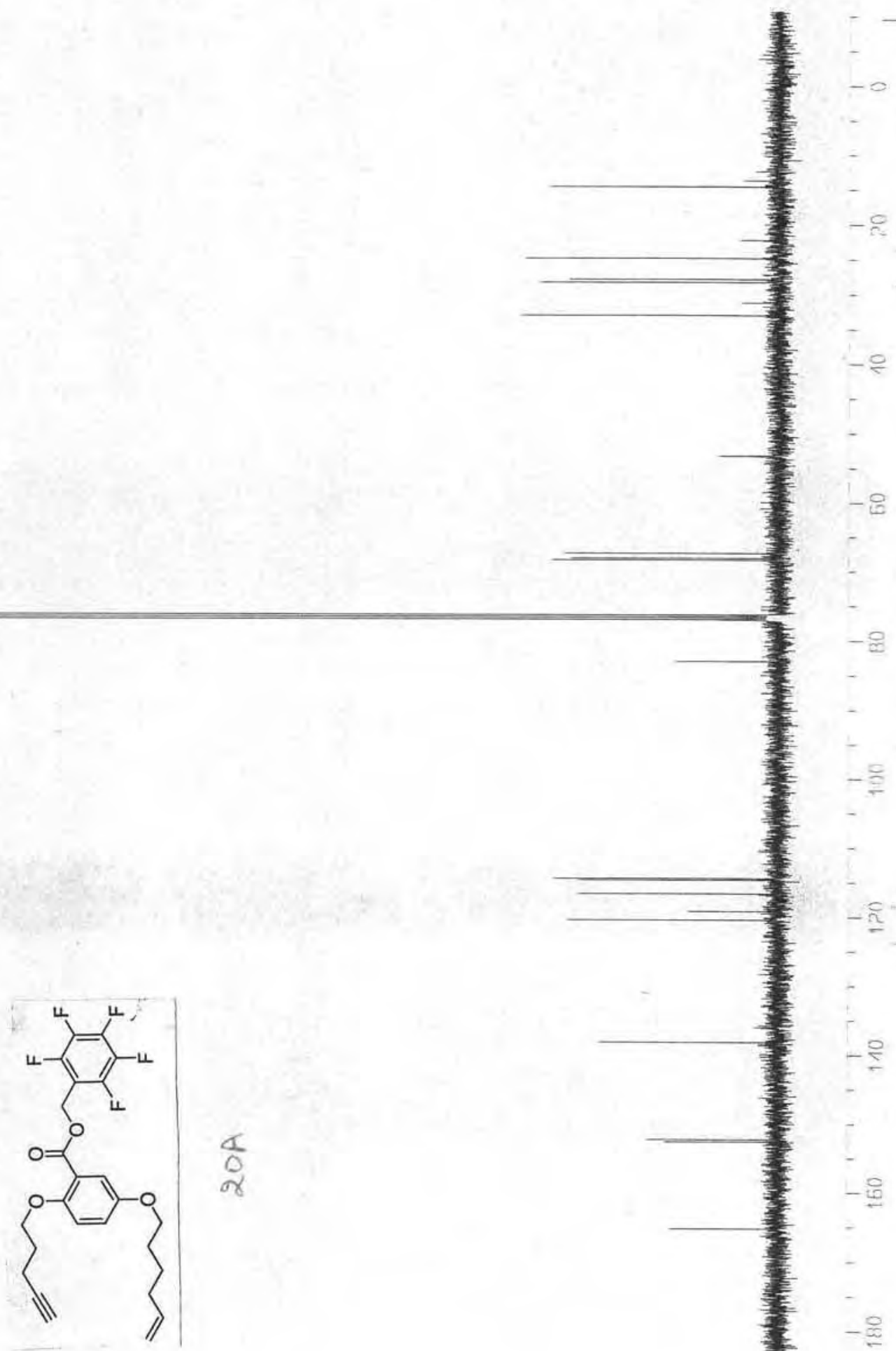

$-0$

$-8$

$-?$ 


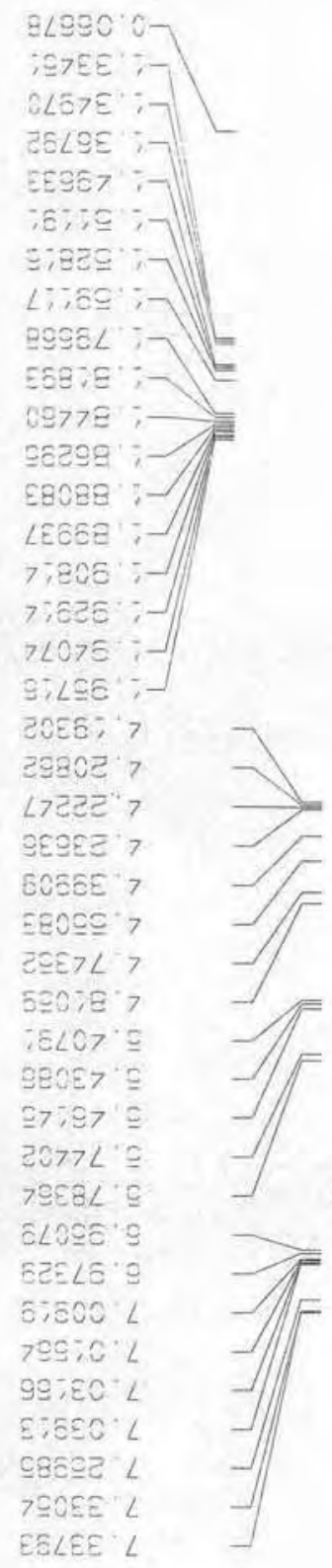

wos
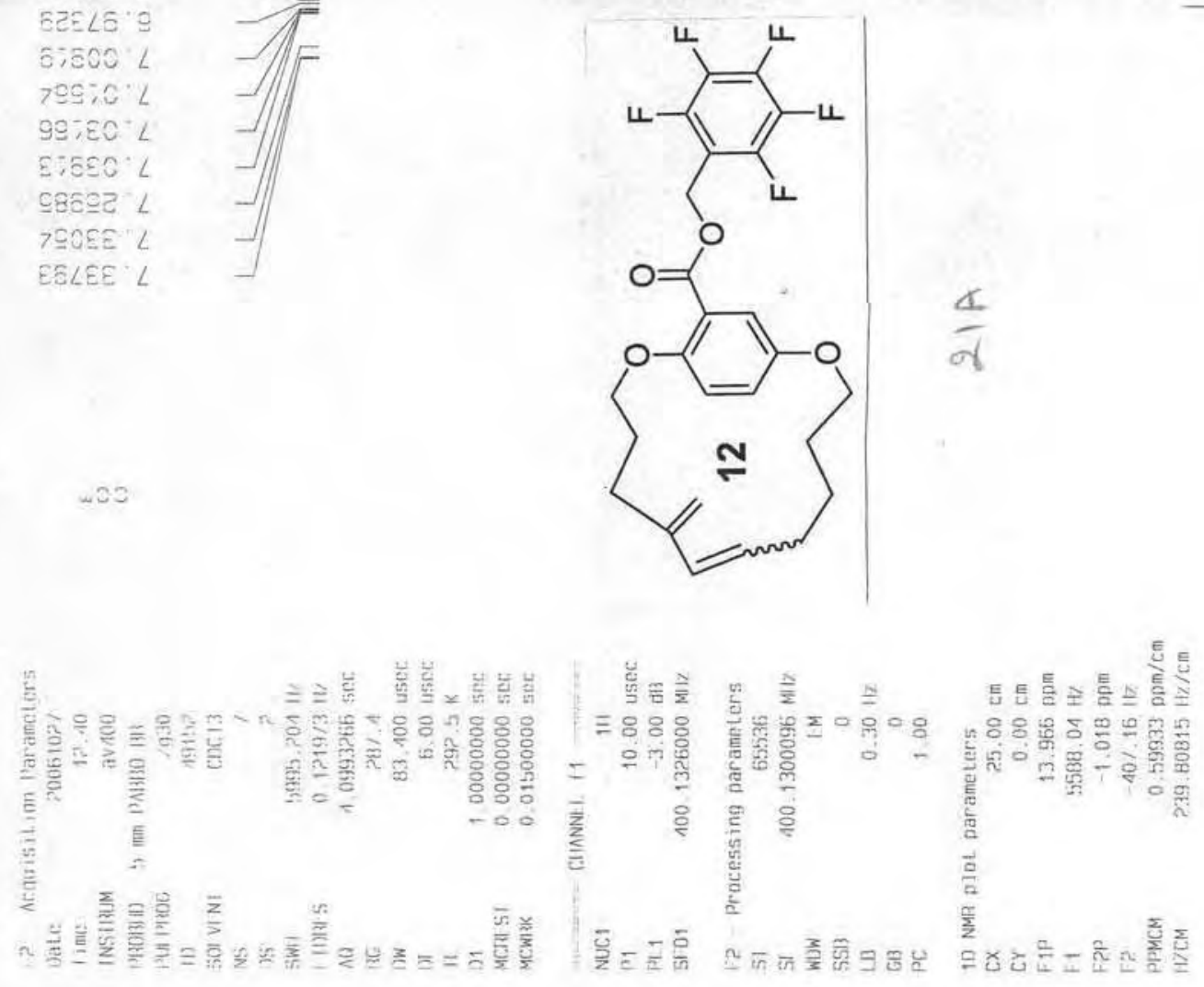

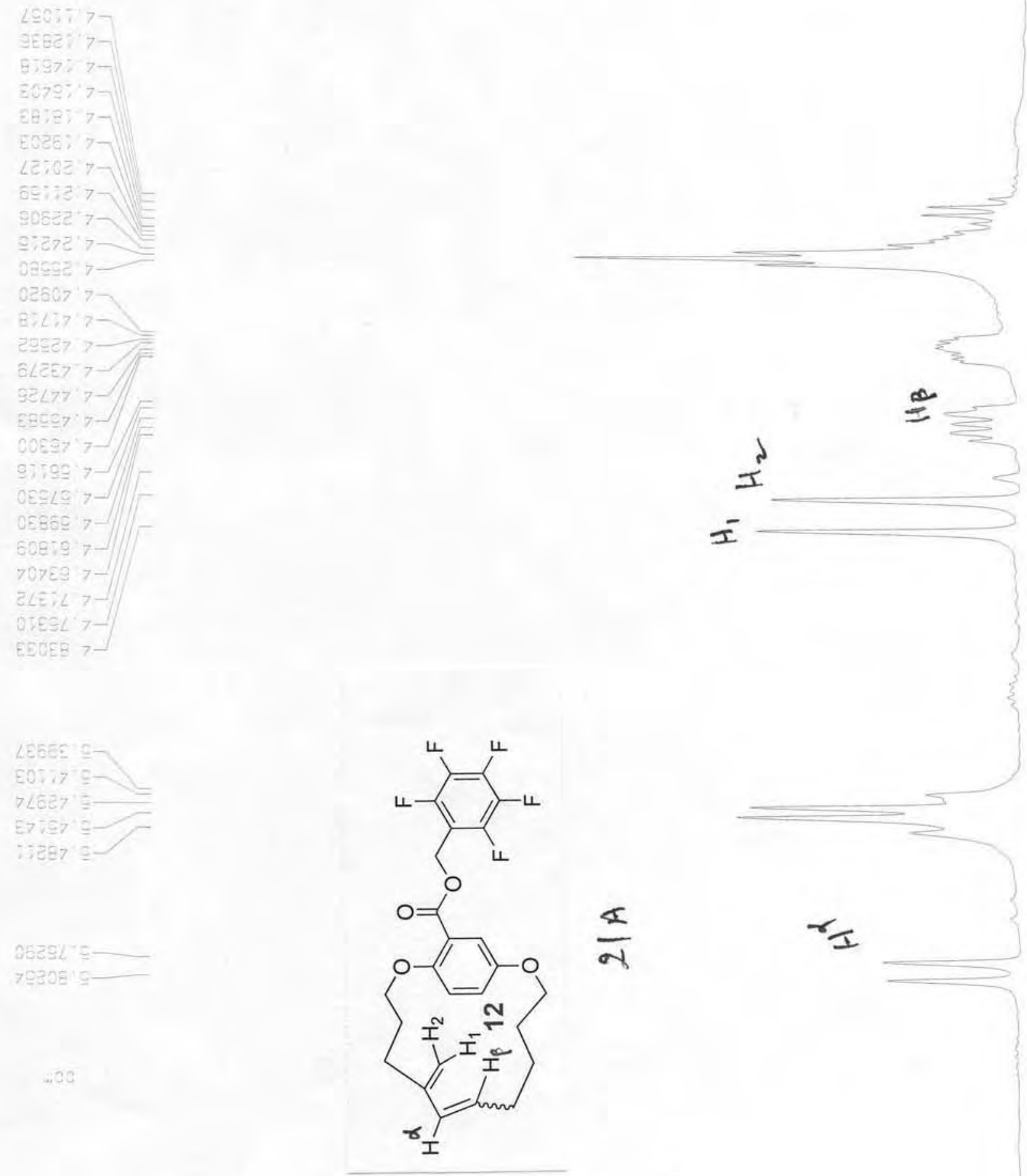

$$
\text { I }
$$

DSट9LY:-
7อ30E

400
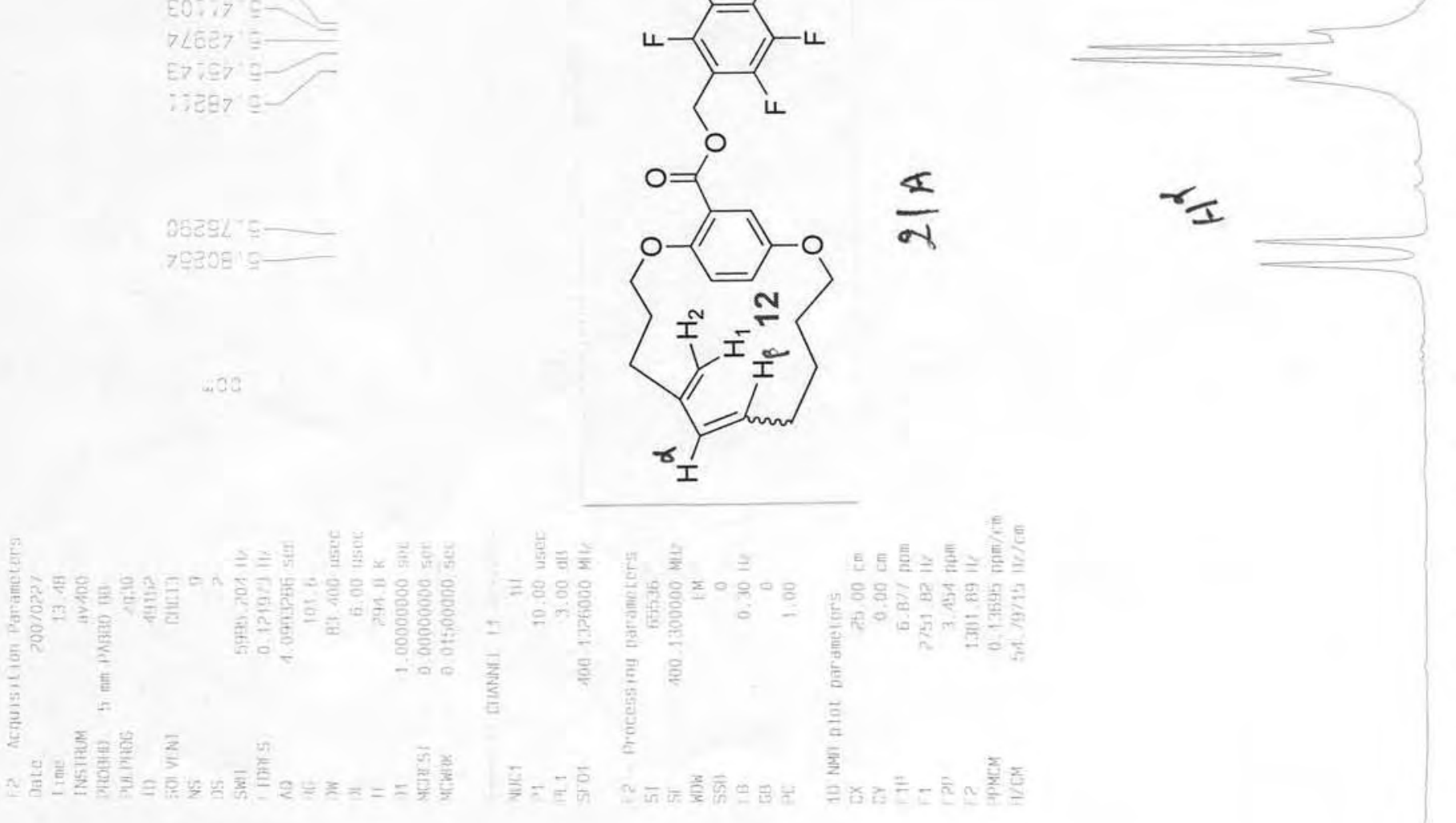


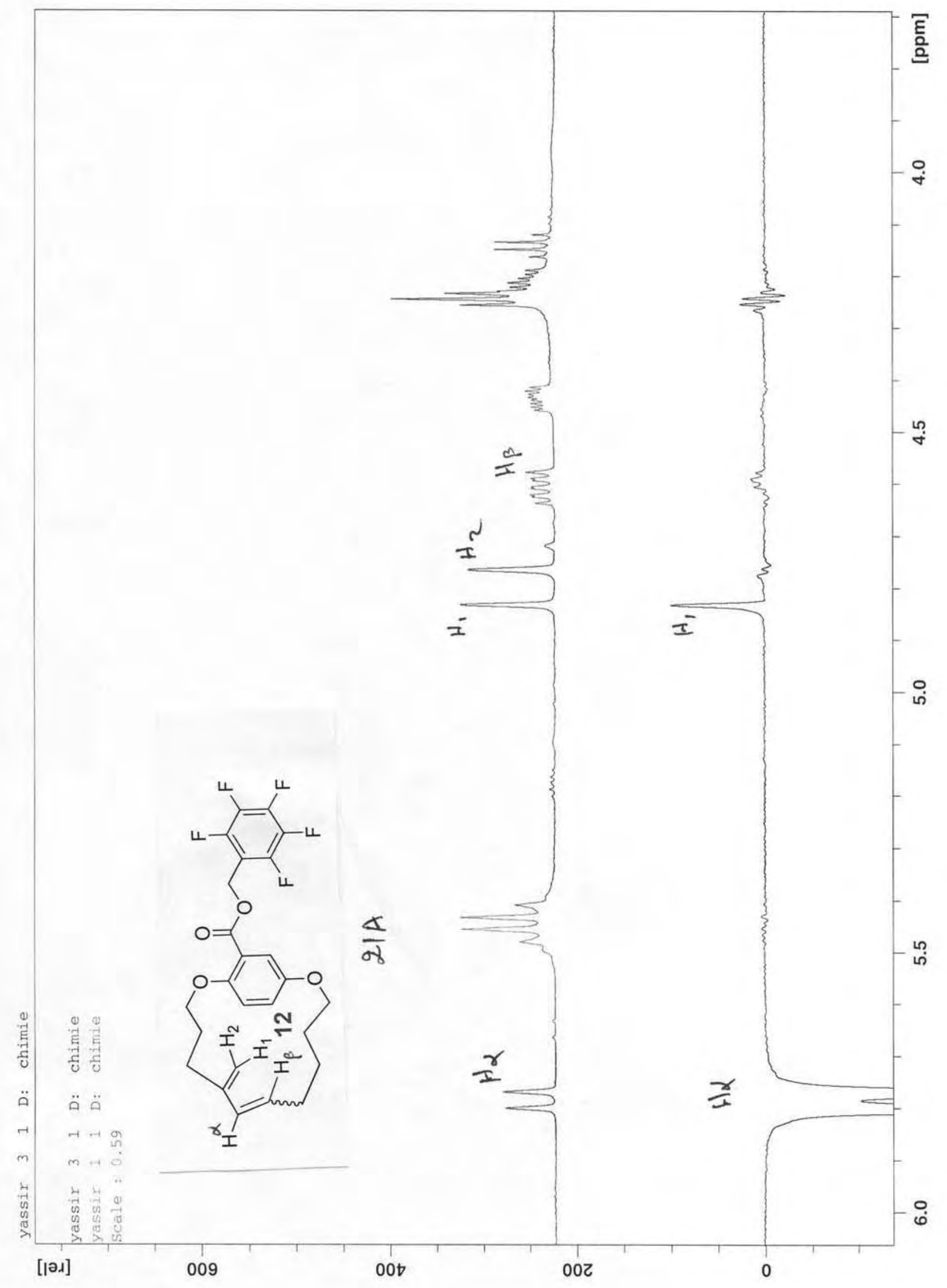


앨 z5

$: 3 L 7 z-1$

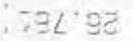

gie le

ize oe

\section{'EEL EE}

BEL $\angle 9$

¿s9 59

IES $8 L$

马65 $9 L$

EIE LL

\section{zoe zi:}

gES हU:

7 SOE $5: 1$

, OED i己;

घडर टट:

'786 हट?

iต; פะ:

ริธ हटँ

हटอ วE:

- 7 \&O EE;

;

E;, 0 ;

EटL टE;
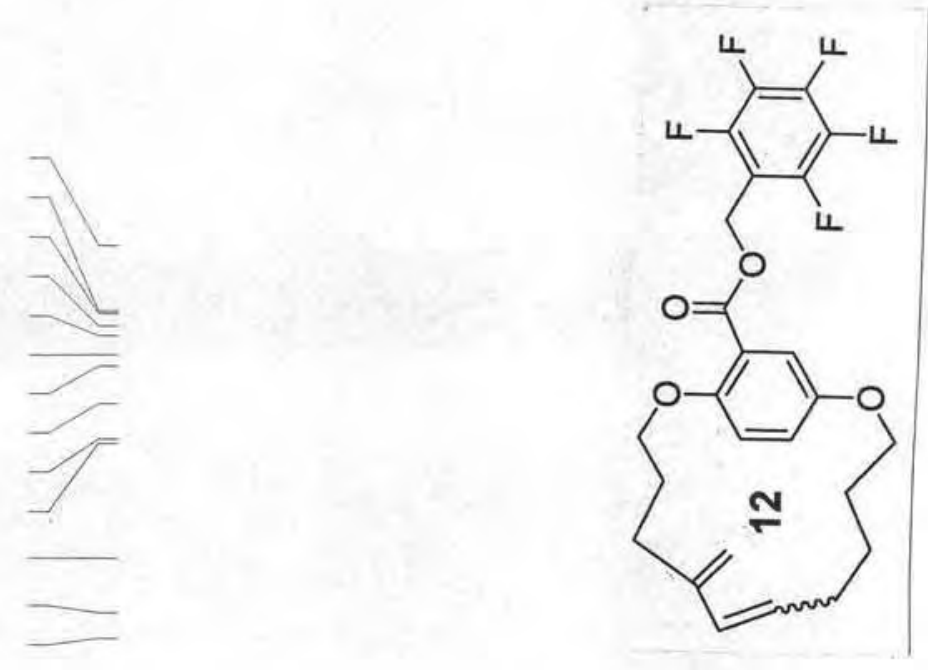

$\frac{1}{a x}$

ร50
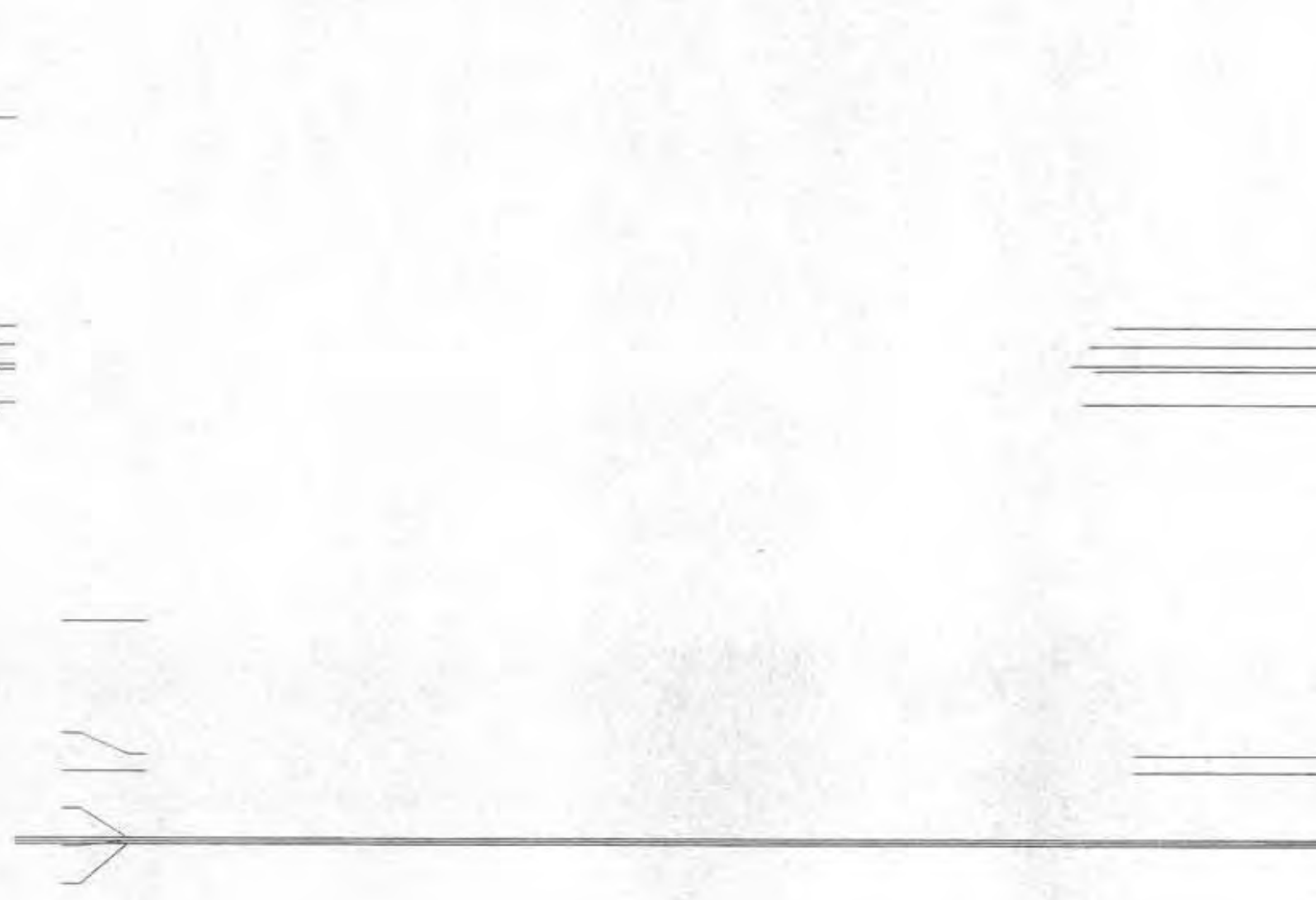


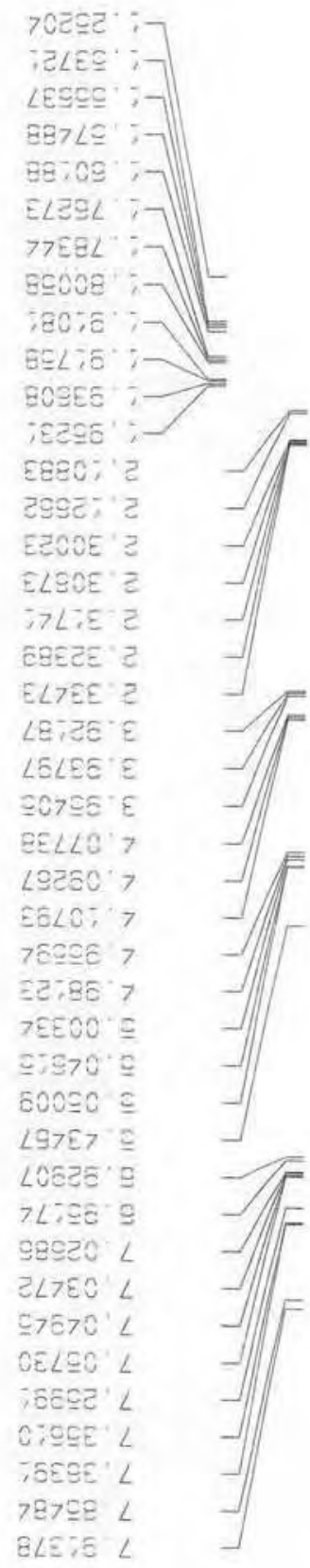

$-53$
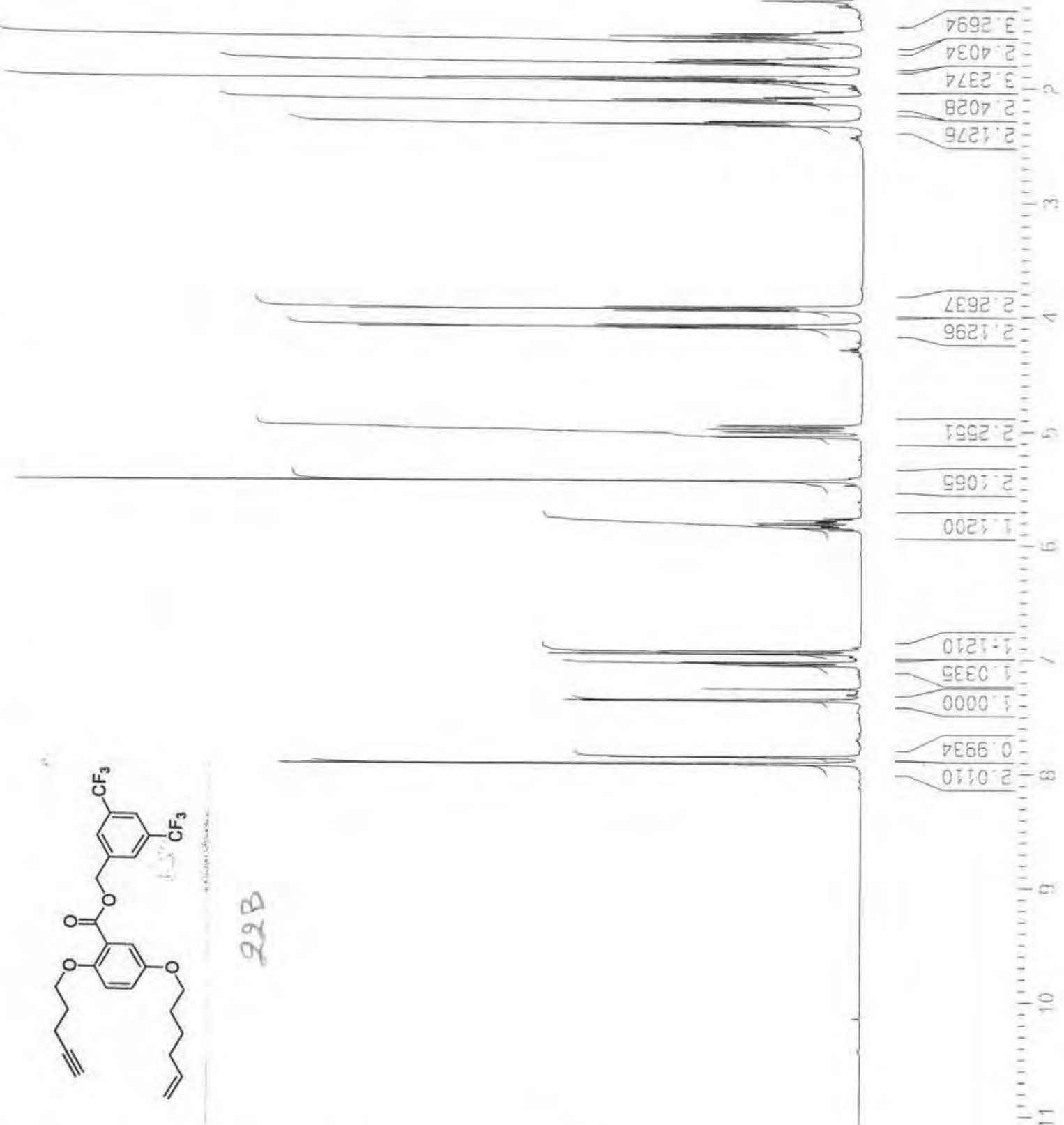

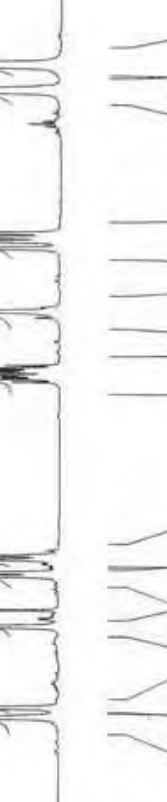

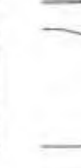


$968>$;
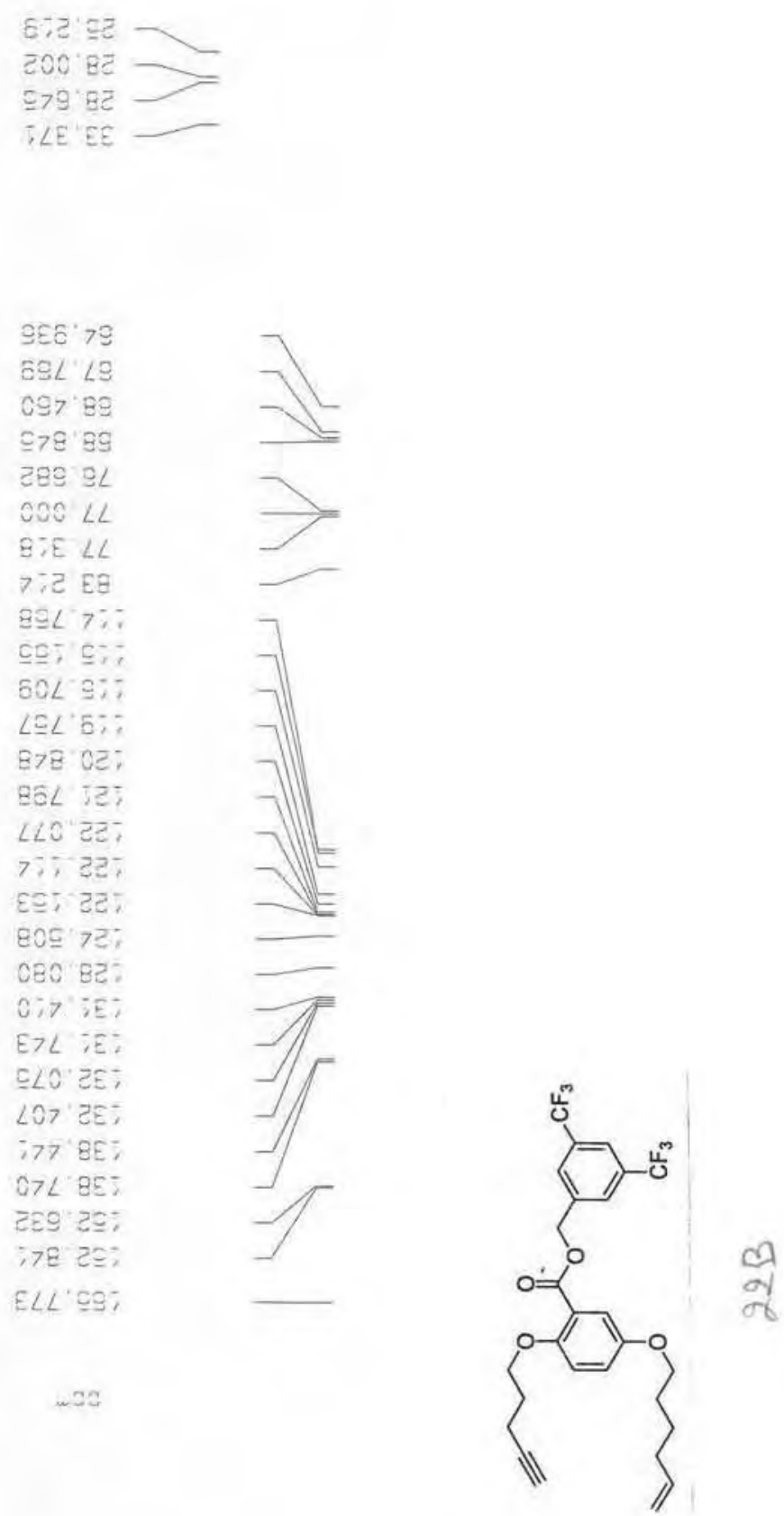

or

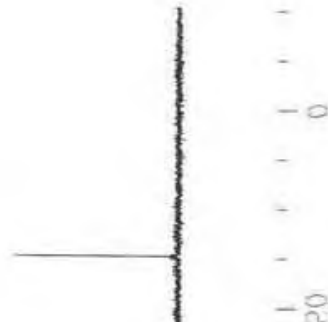

$(3$

078 to

,LE SE

टอS $3 L$

$000 \angle L$

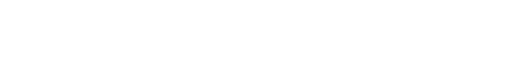

$-0$

$-\infty$

$-$

- 우

$-$

$-8$

$-$

$-\infty$

$-8$

-

$-\underline{2}$

8 告

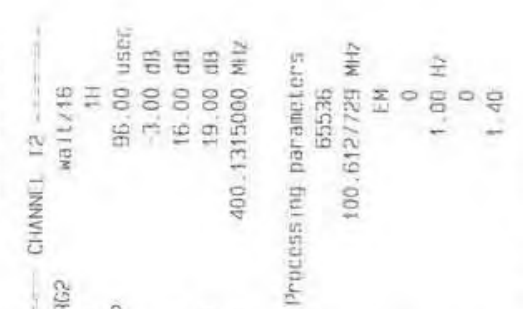

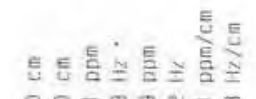

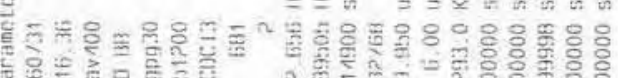

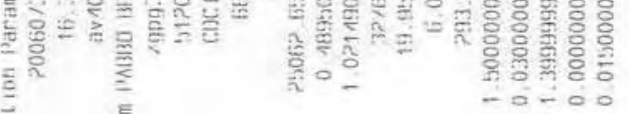

正

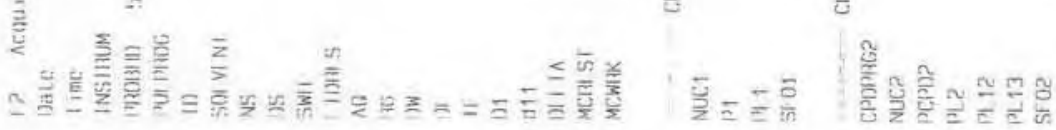

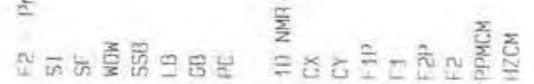
으음 


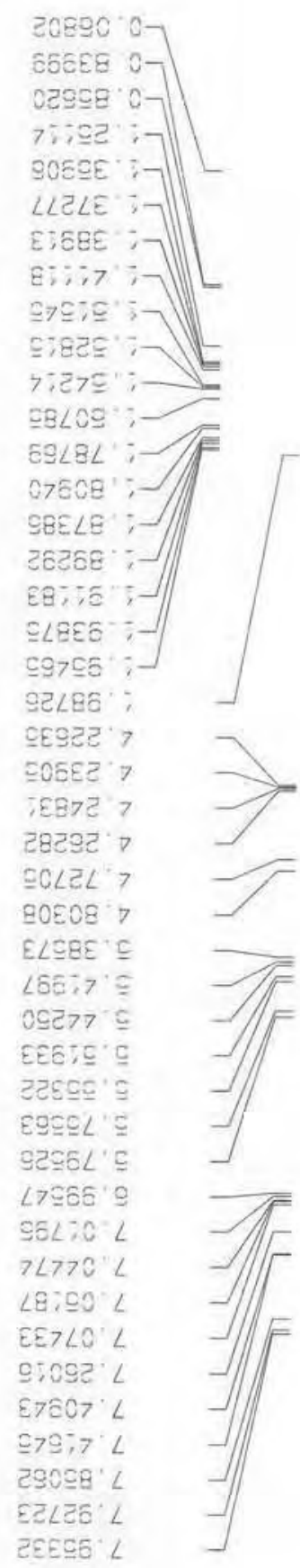

4.5e

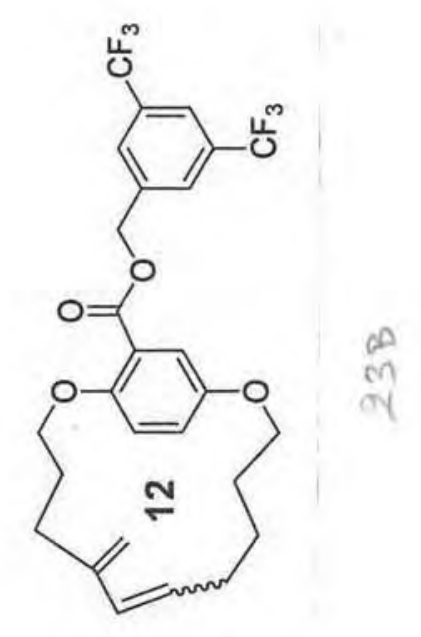

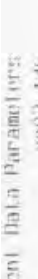



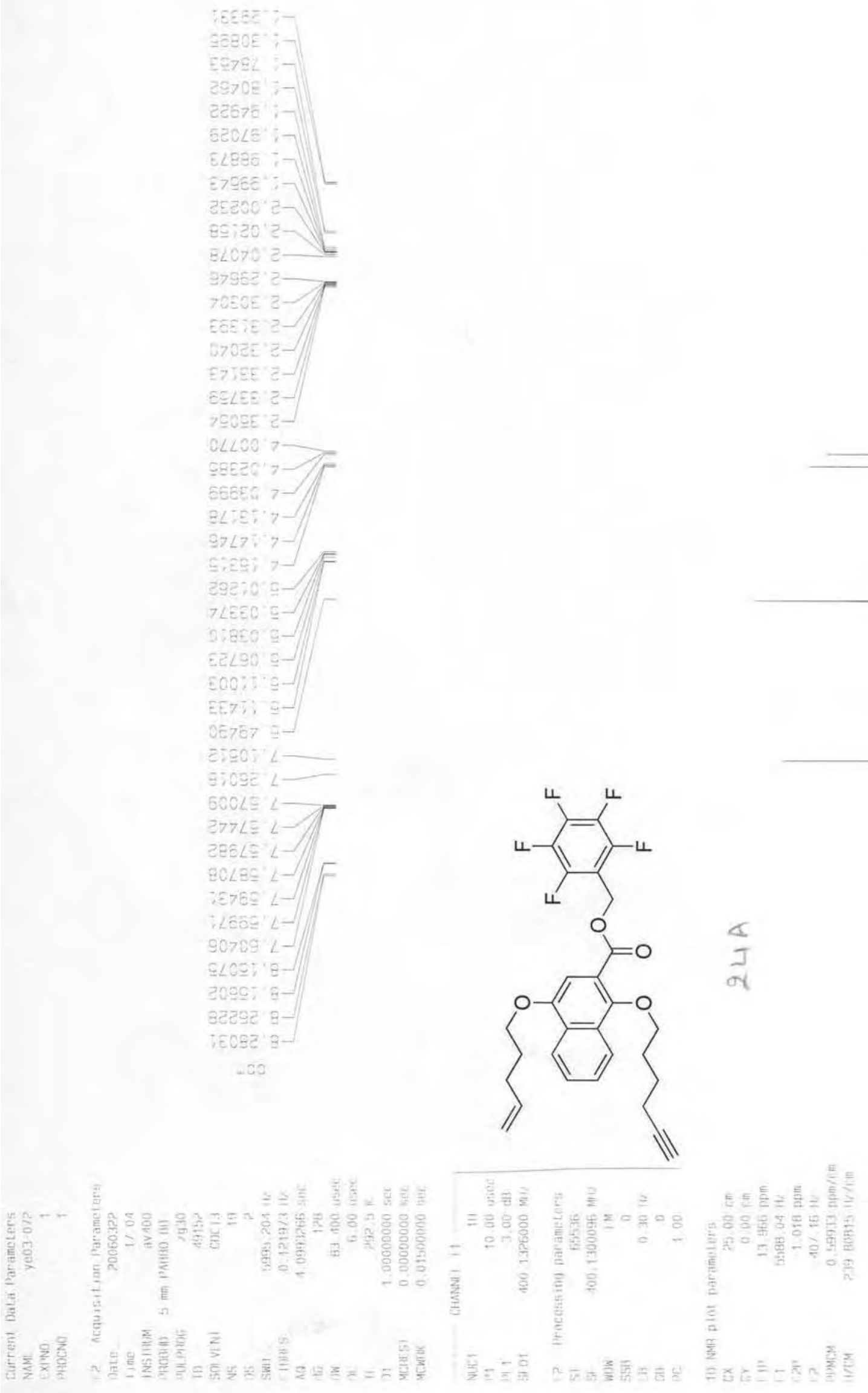
gEE' G:

$760^{\circ}: 33-\longrightarrow$

gCE be

टEE 'ह

gas 05

EE6 Eg

$07 E \angle 9$

709.99

089 t

उ9g: 94

000 LL

LiE LL

LC5':

$280^{\prime} 70 \div$

66е

Dope Li:

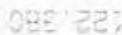

SC7 EZ:

TOF' $L E$ :

टश5 LZ:

og: $52:$

टละ

S:LLE

פELL' 39

c98 is

IอL g9:
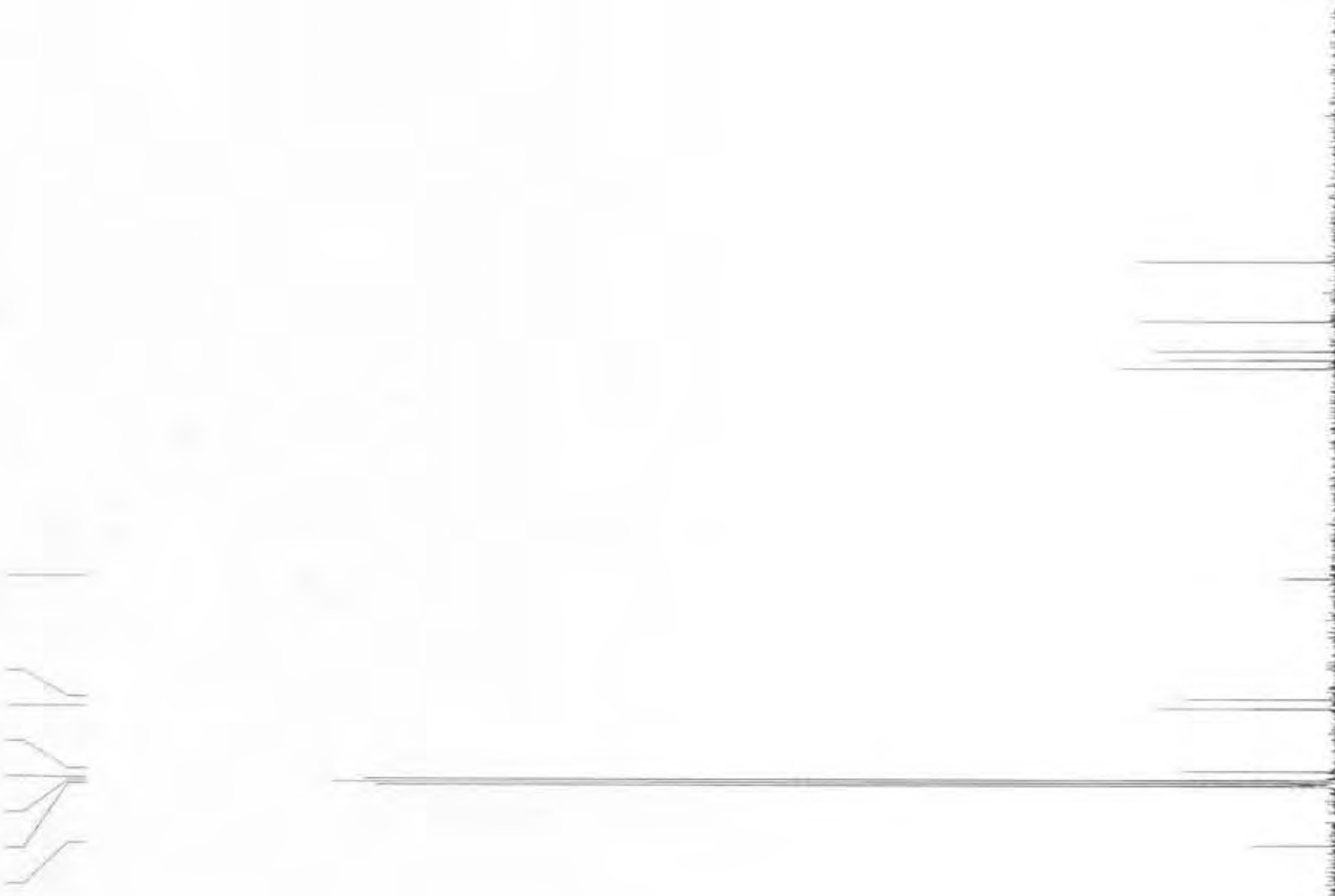

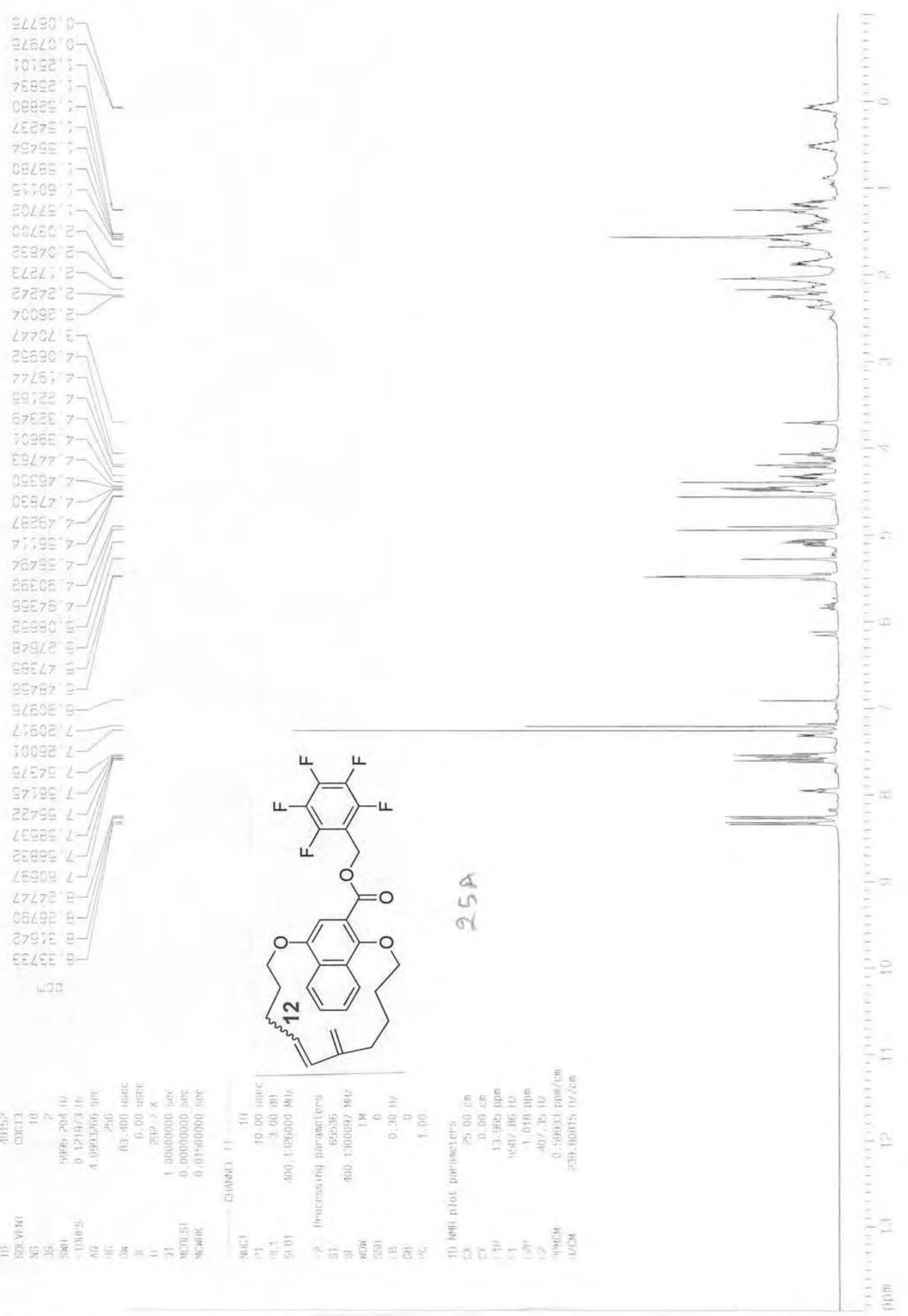


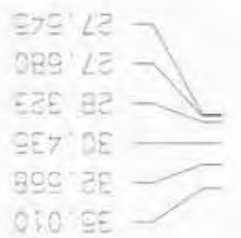

उSE :3

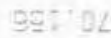

aco'el

5eg $9 L$

666 $9 L$

$700^{\circ} \angle L$

LIE LL

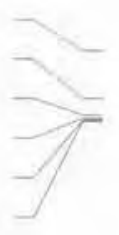

Gog'so:

Ege' or

$7759: \%$

यद्र ?2:

OET 7E:

:

द: $21 \mathrm{se}$

GLE GE:

उद7 ह5:

zER 5a:

OER OE:

$90 \mathrm{~L}$ IE

769.97

79; 09:

$5 \angle 7 \quad 7:$

E96 이

iog'se:

wos
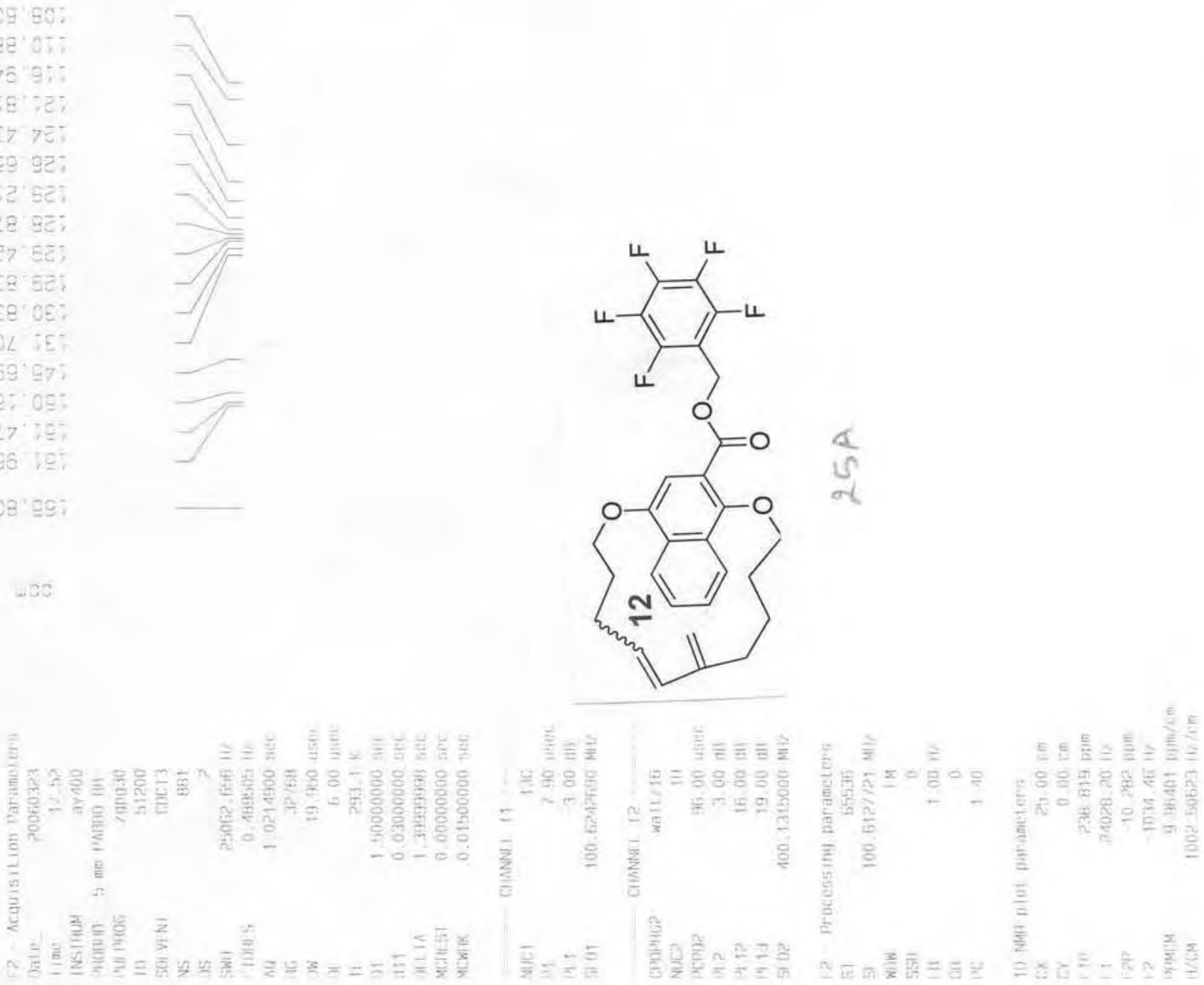


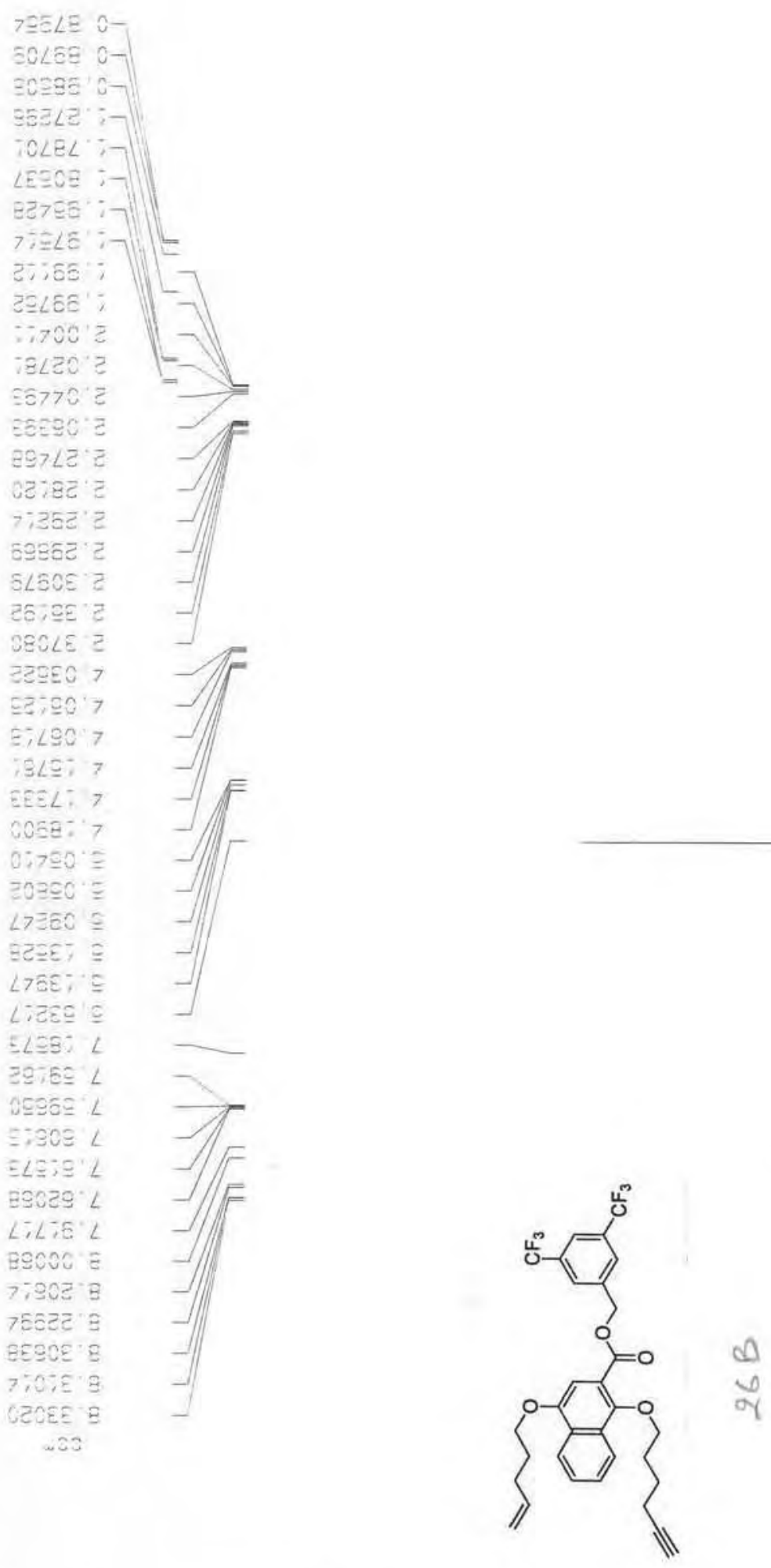



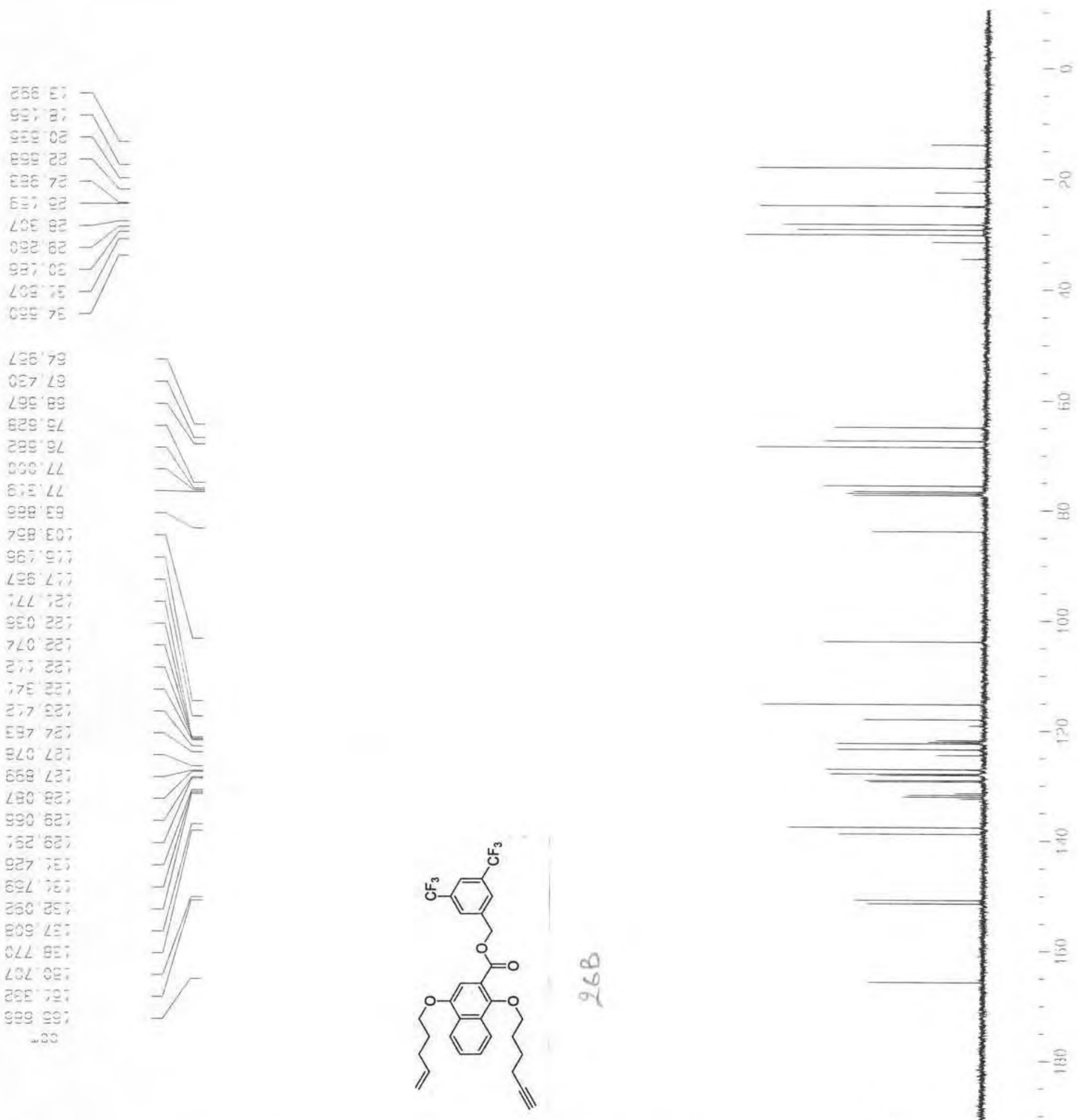

$-8$

$-9$

$-\underline{0}$

$-\underline{1}$

$-\underline{2}$ 


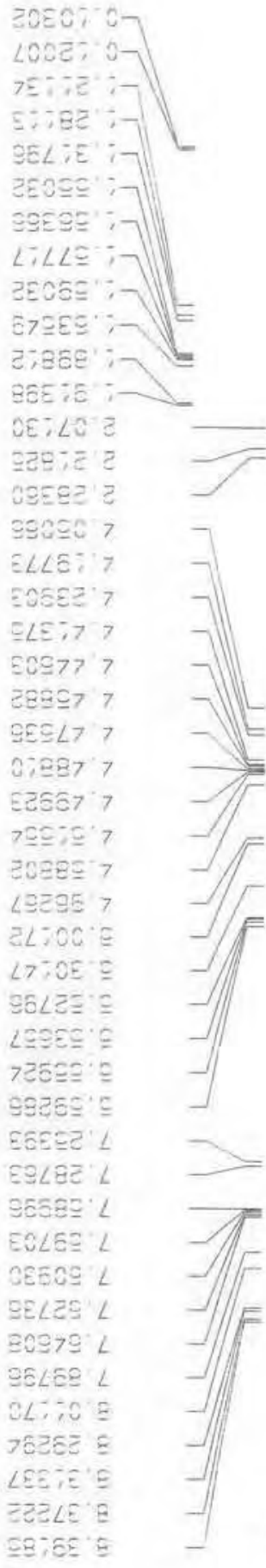

- 30

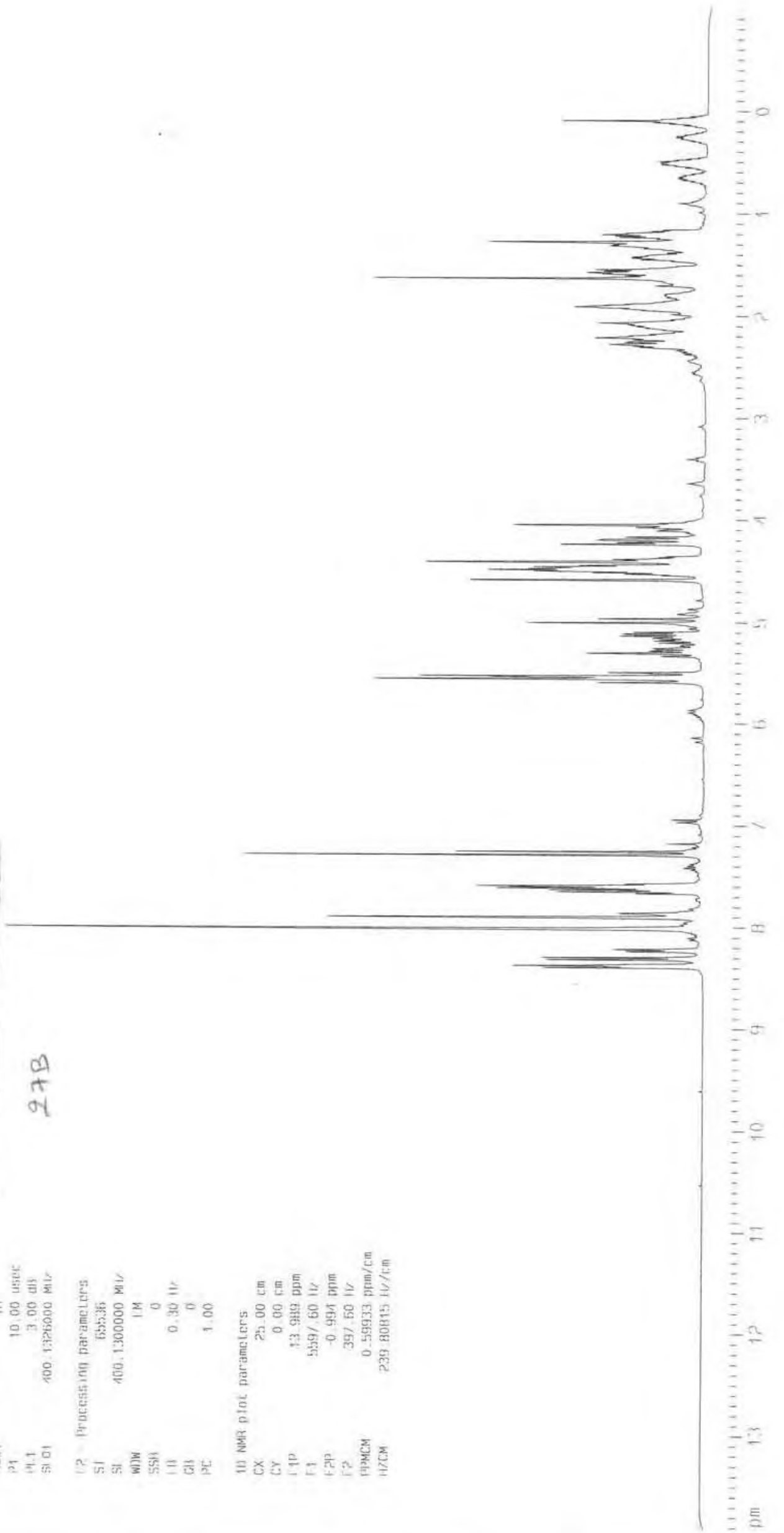



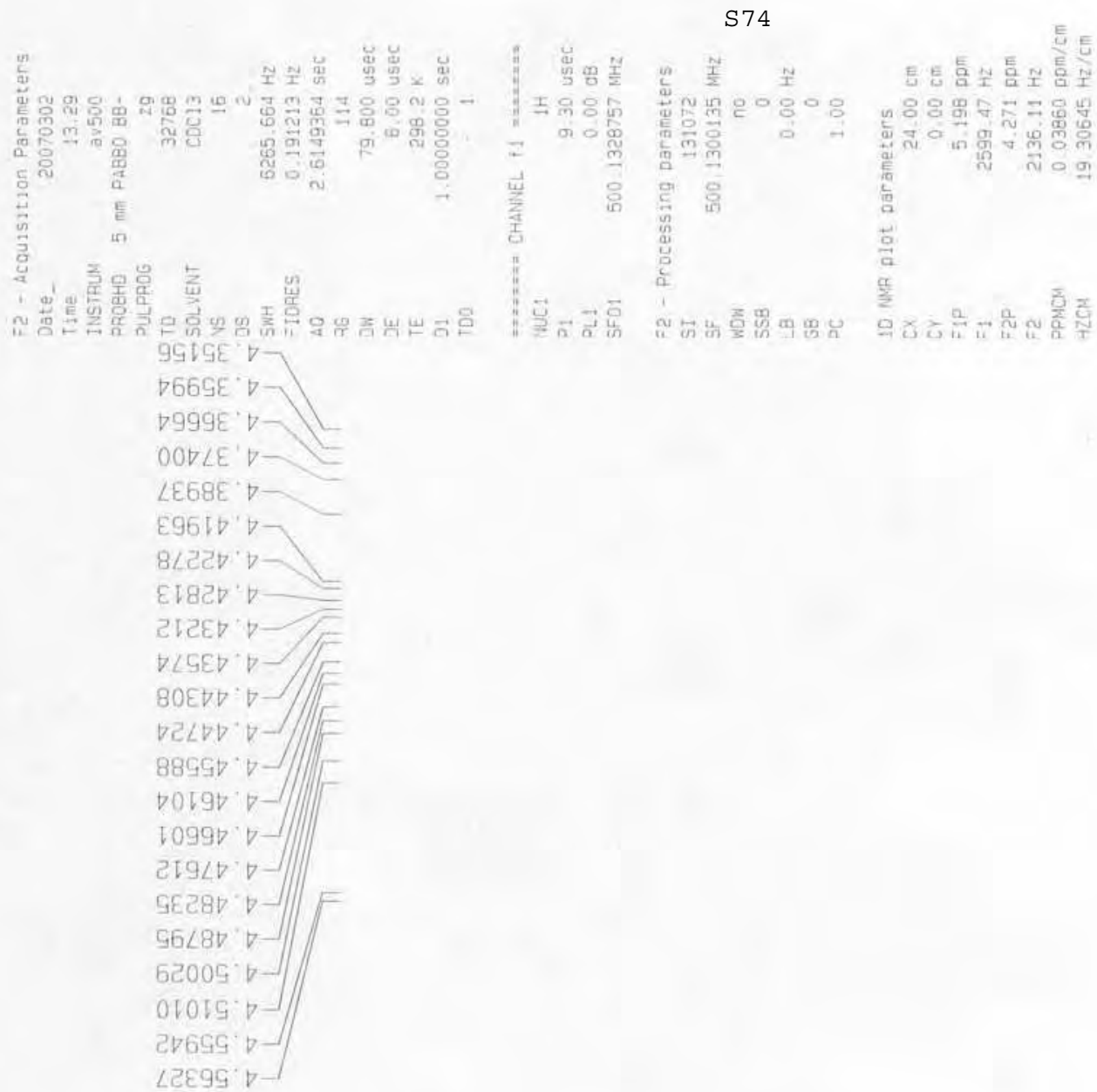
9GIGE ' ग66GE $007 / E^{\prime}$

$\angle E 68 E^{\circ}$ 岁 $x$ 㟧

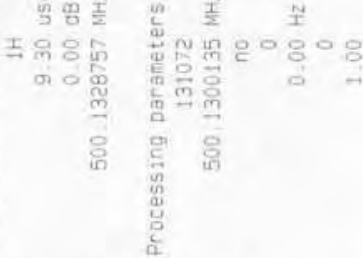

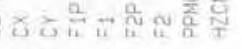
E96 ID हा8टन टाटEन $\nabla-$ $\checkmark \angle S E D D^{\circ}-$ BOEDV $\square-1 /[$ 889G7 $\nabla-1$ $56 \angle 80^{\circ} \circ$ бटО0द टจ6SG จ $\angle$ Leg9 $\nabla-$

$88156^{\circ} \circ-$

$\nabla 6286^{\circ} \mathrm{r}-$

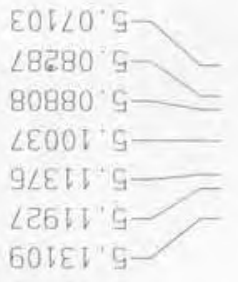

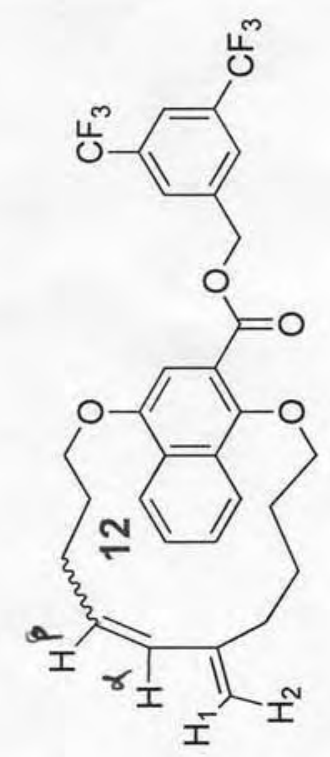

$\infty$

it

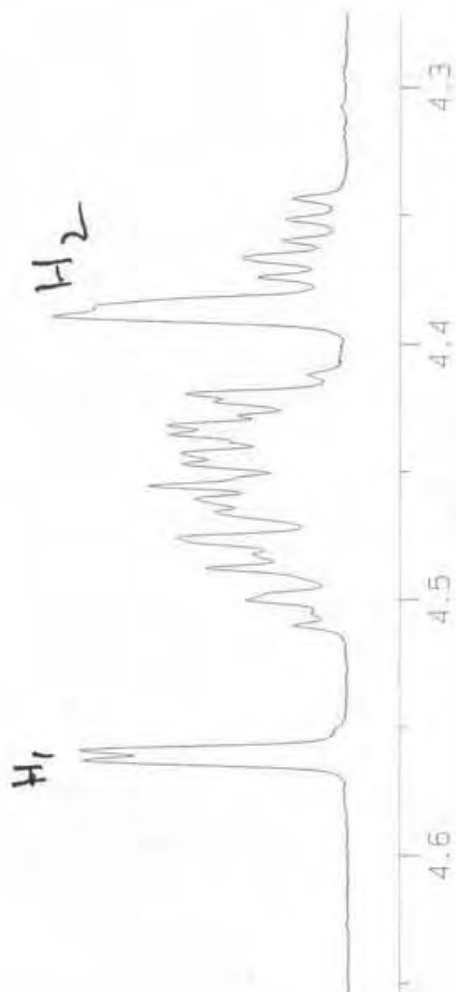

$\checkmark$

$\infty$

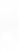

o! 


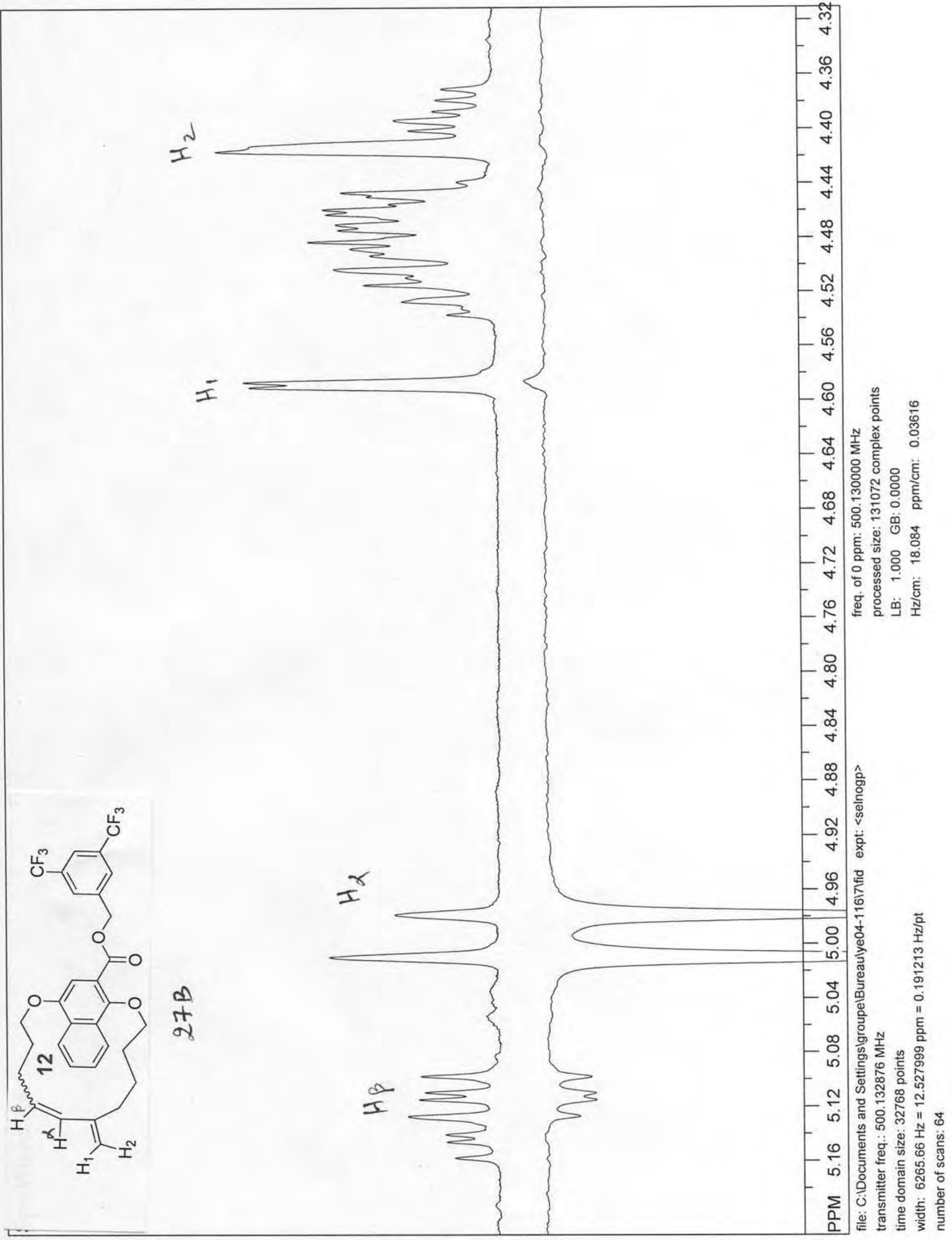



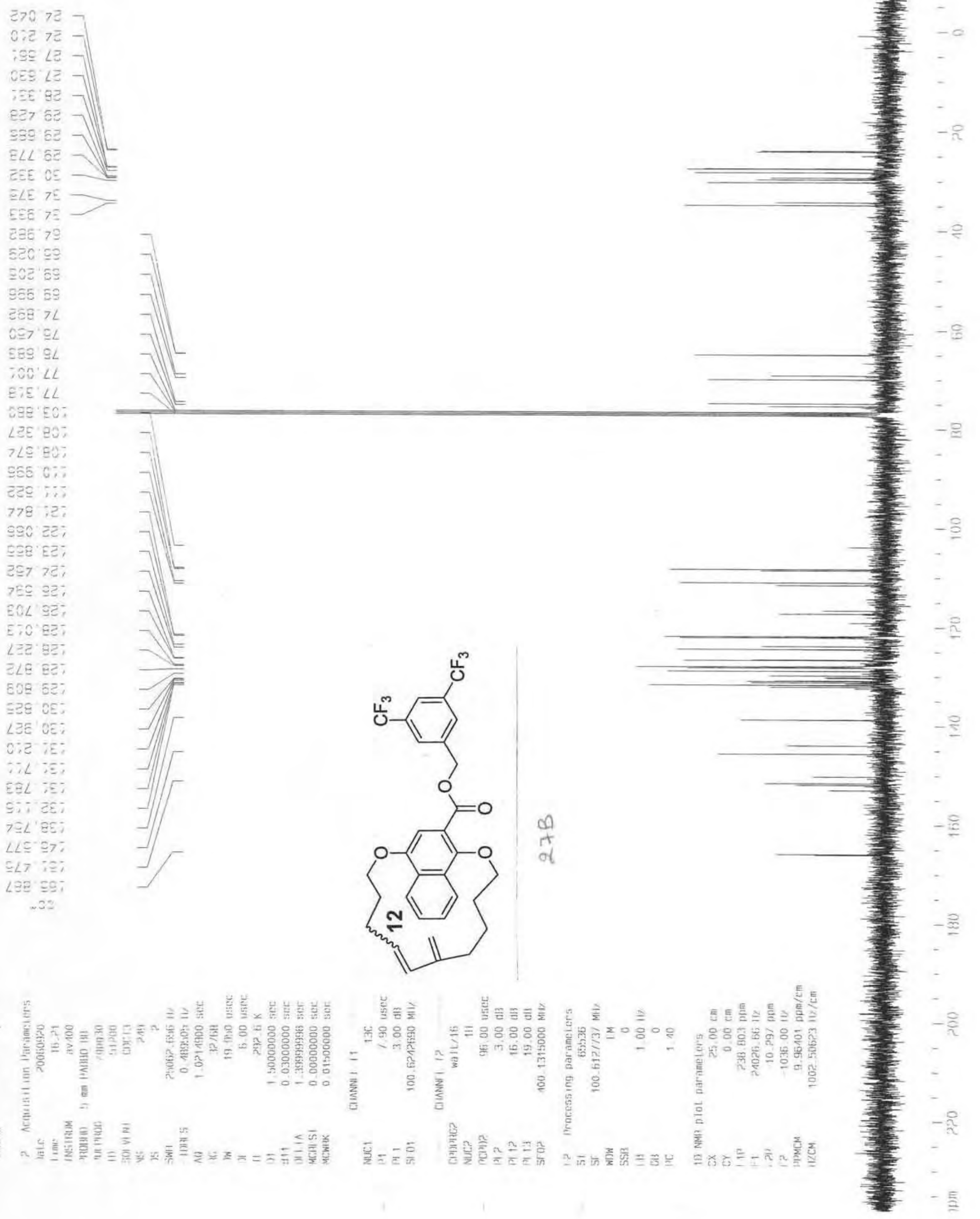

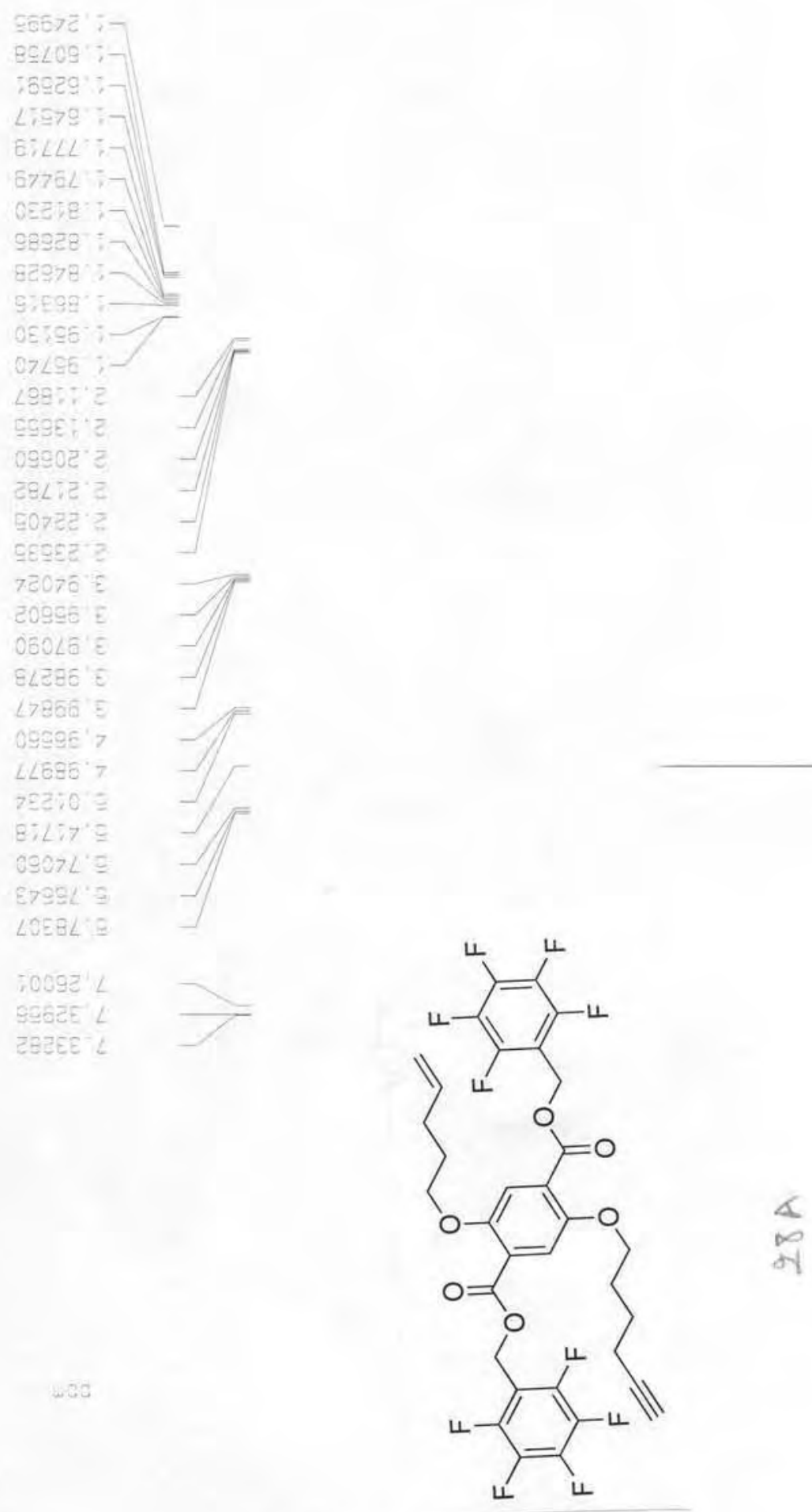

400

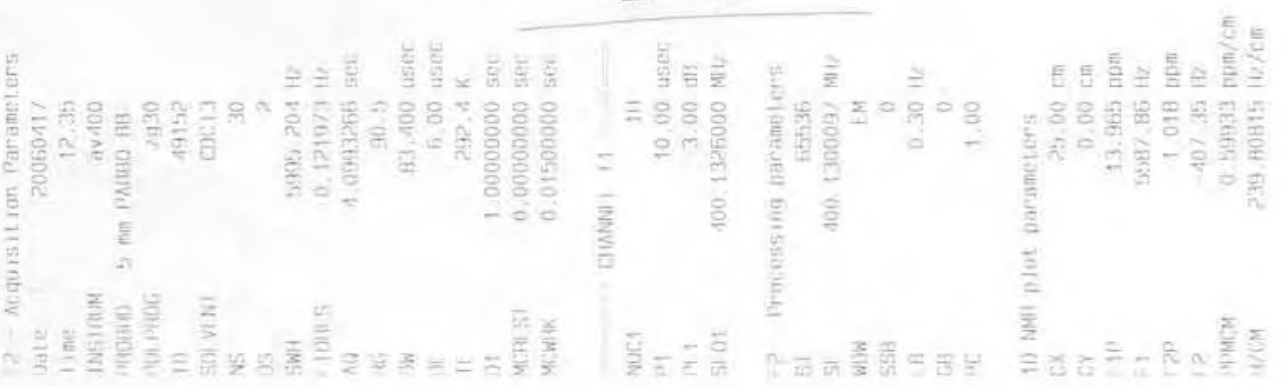


70079

96อ 89

$27 L$ ' 99

396 $8 \mathrm{~s}$

TES $9 L$

65E. $9 L$

LIE LL

ELLEO
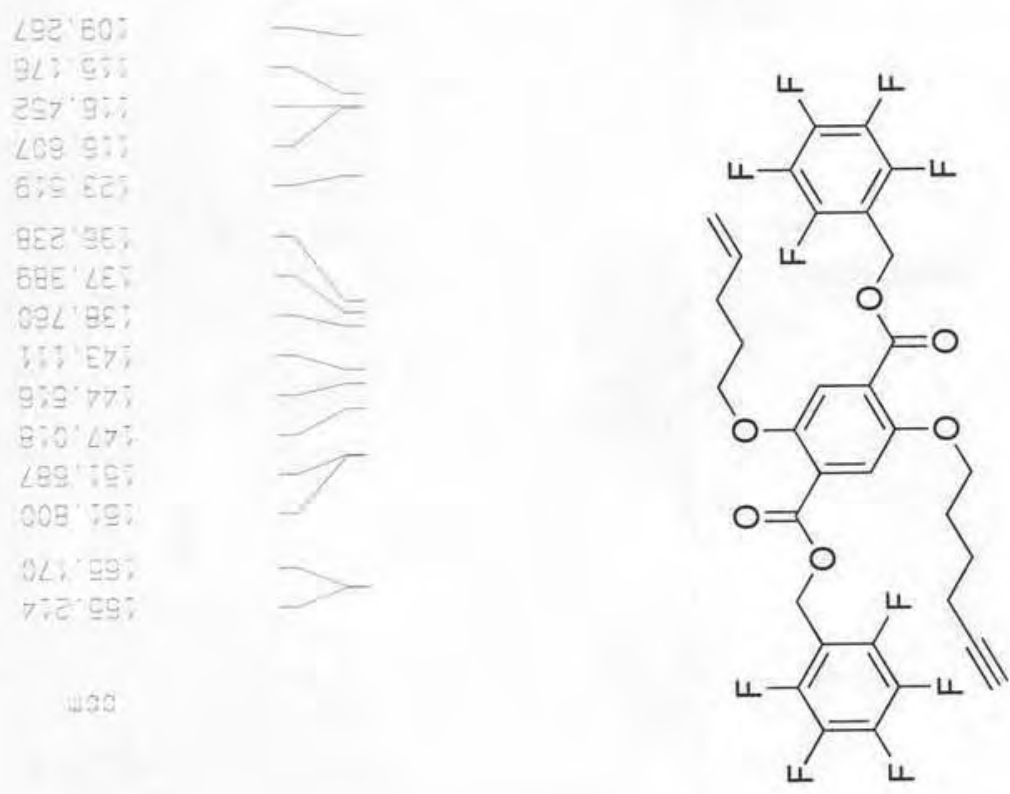

$\sum_{\infty}^{\infty}$

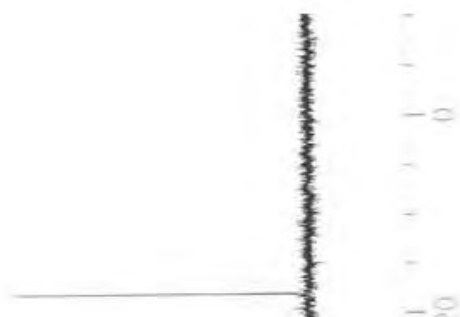

$-\stackrel{ }{5}$

\&
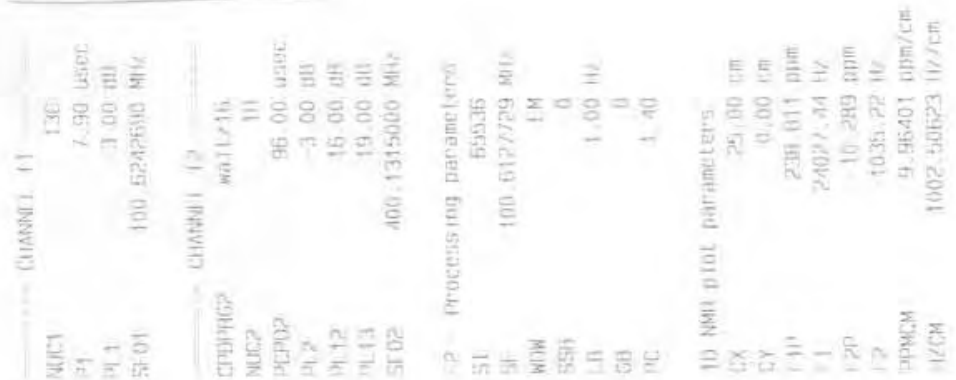

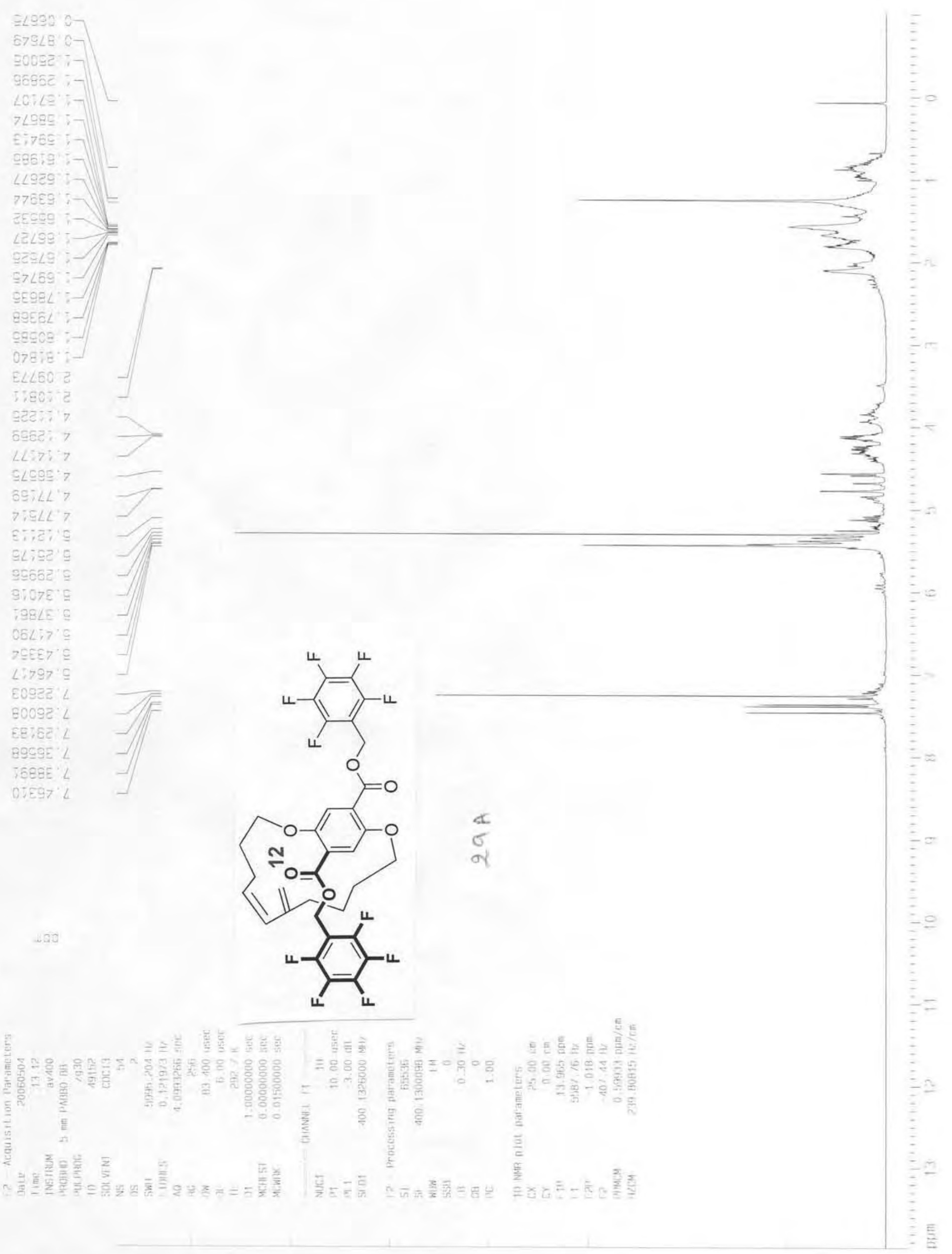

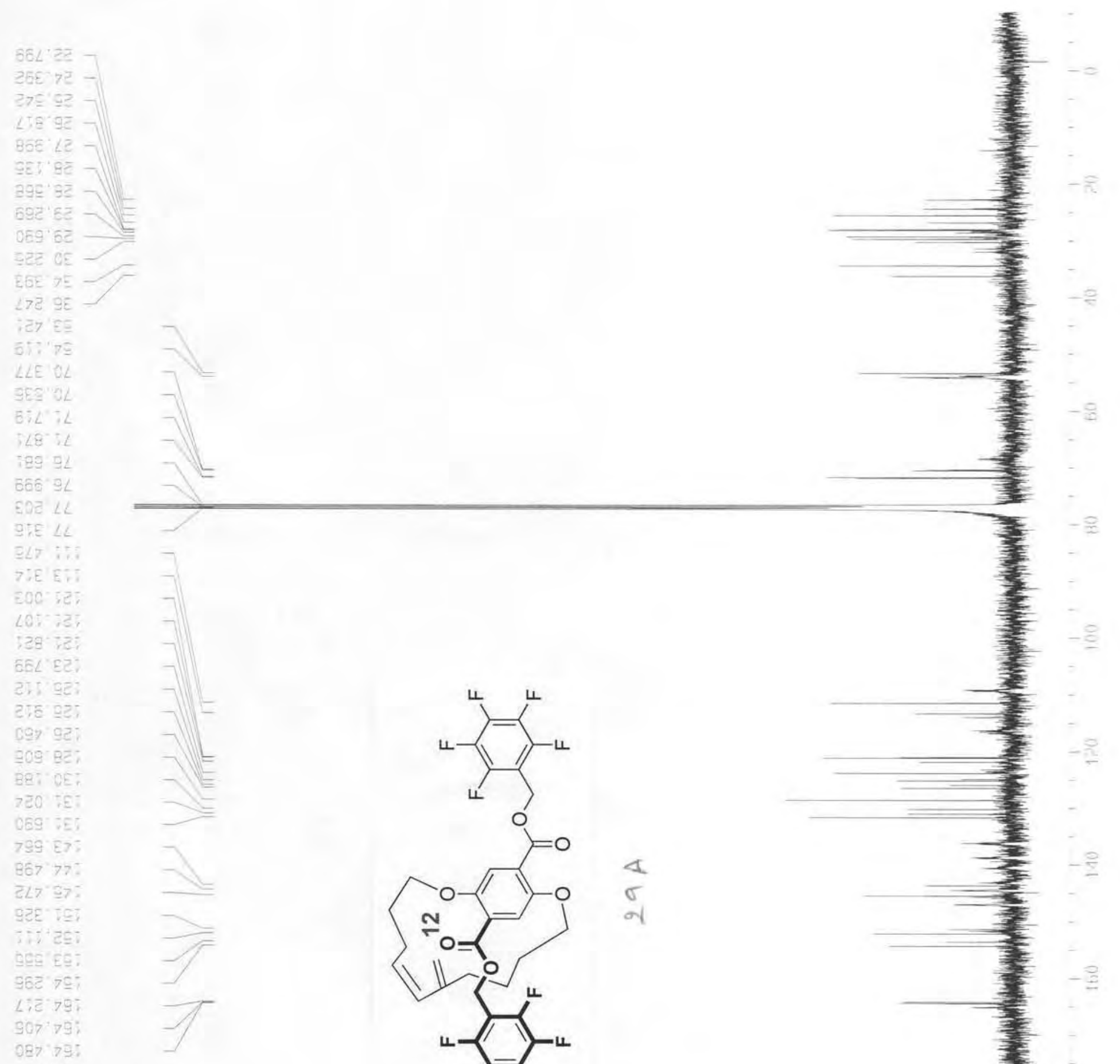

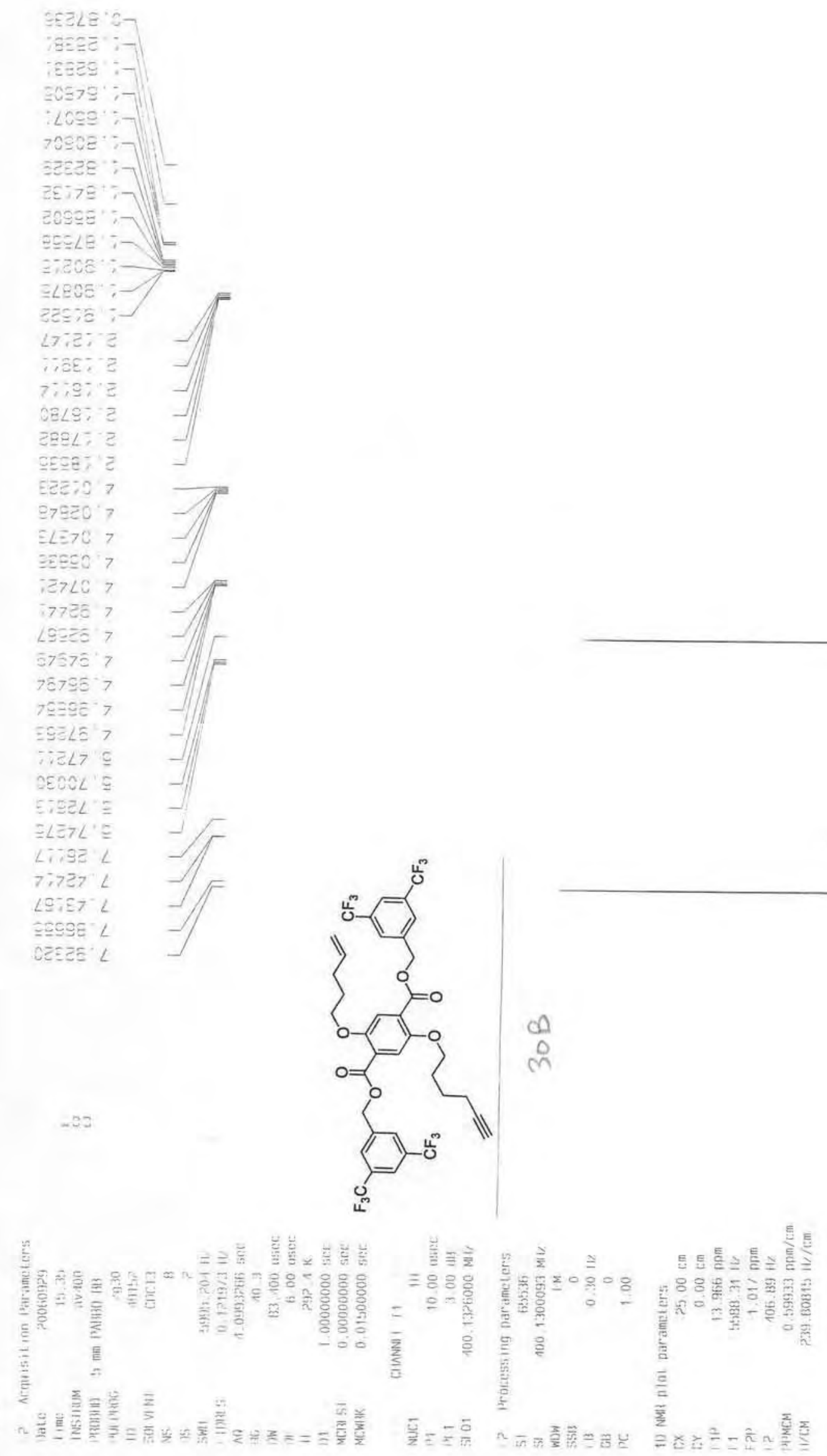

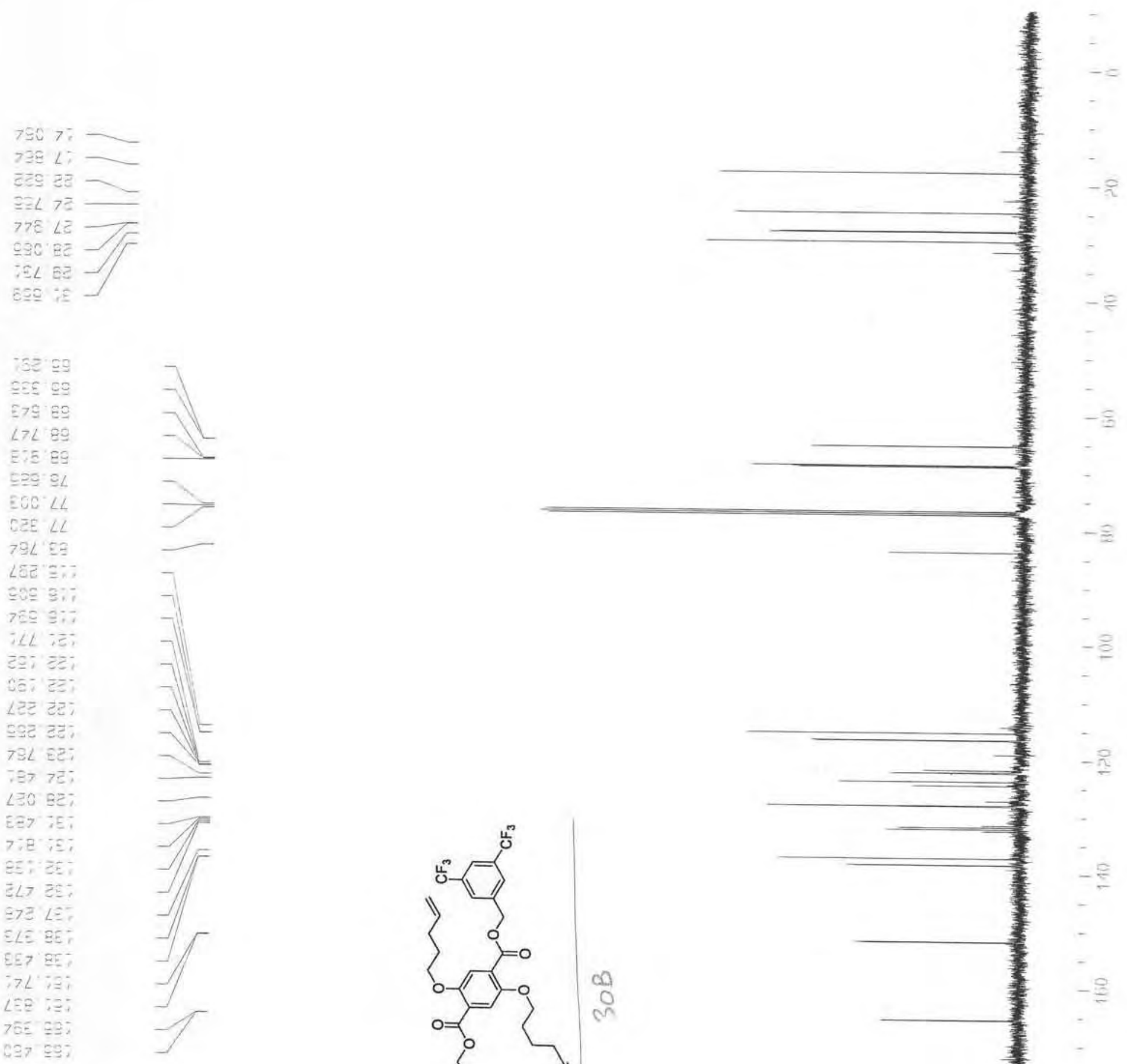

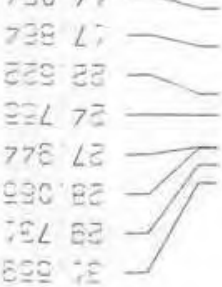

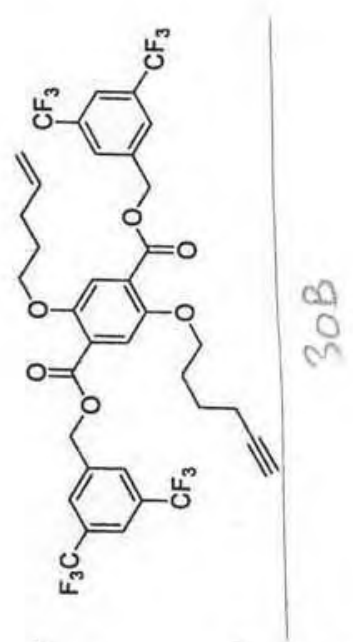

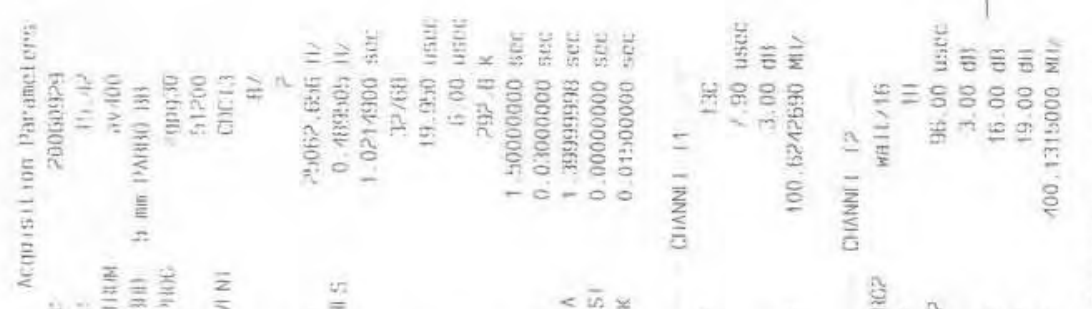

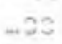

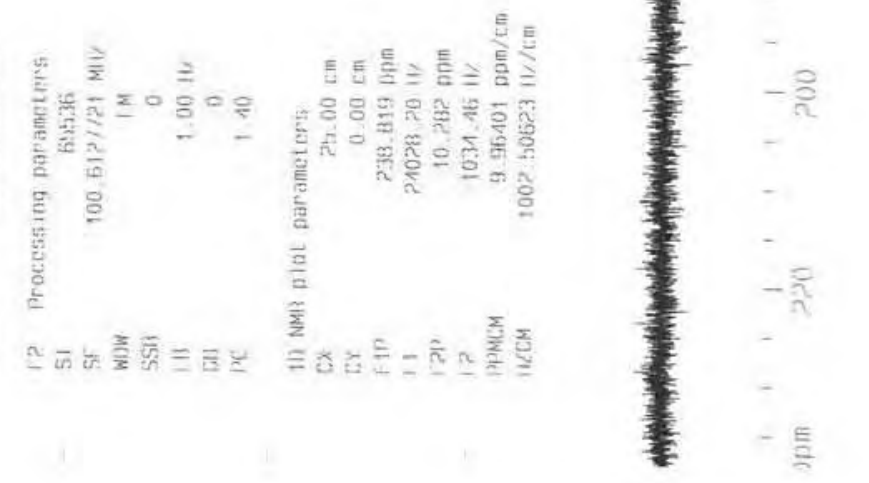



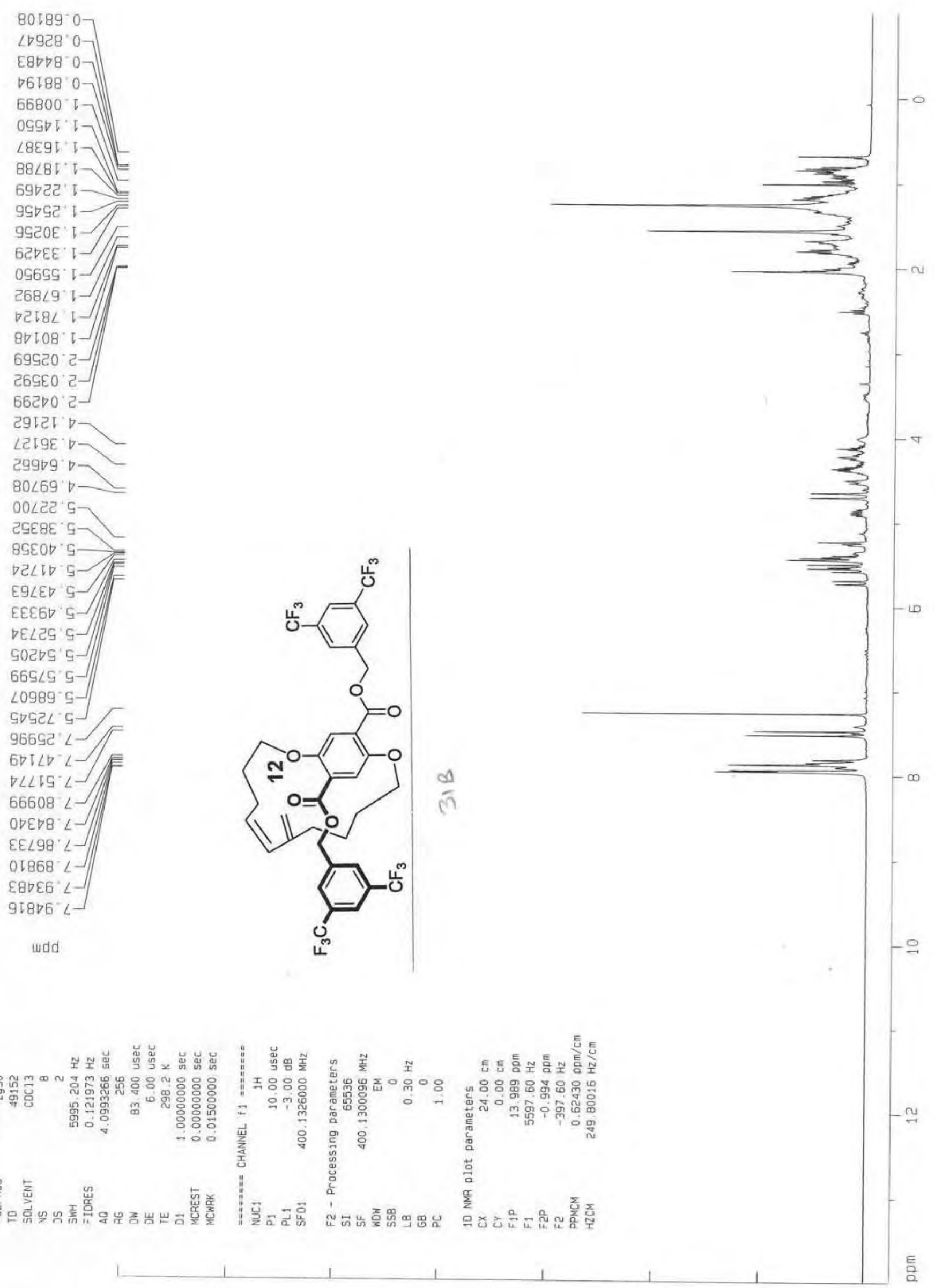

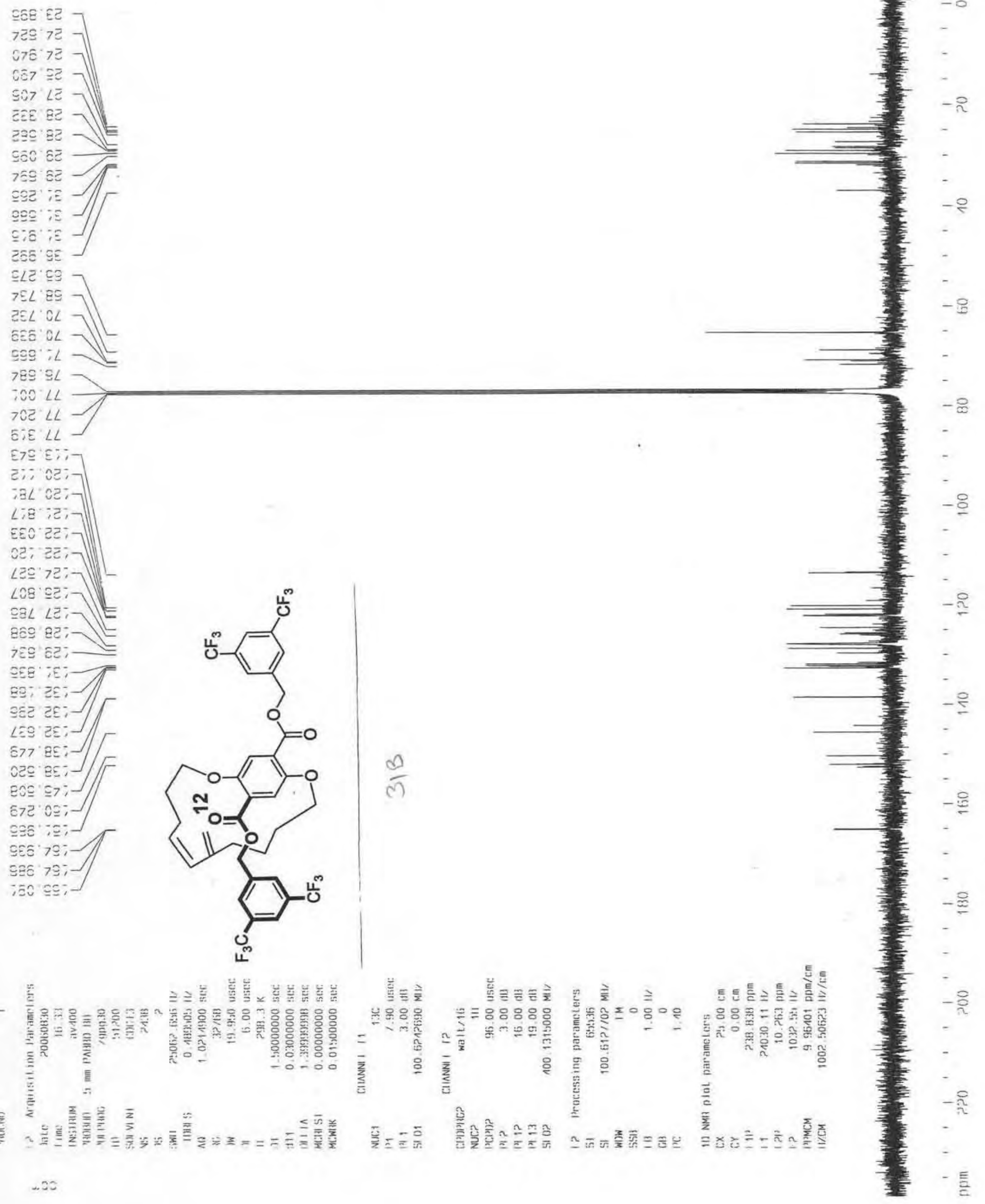

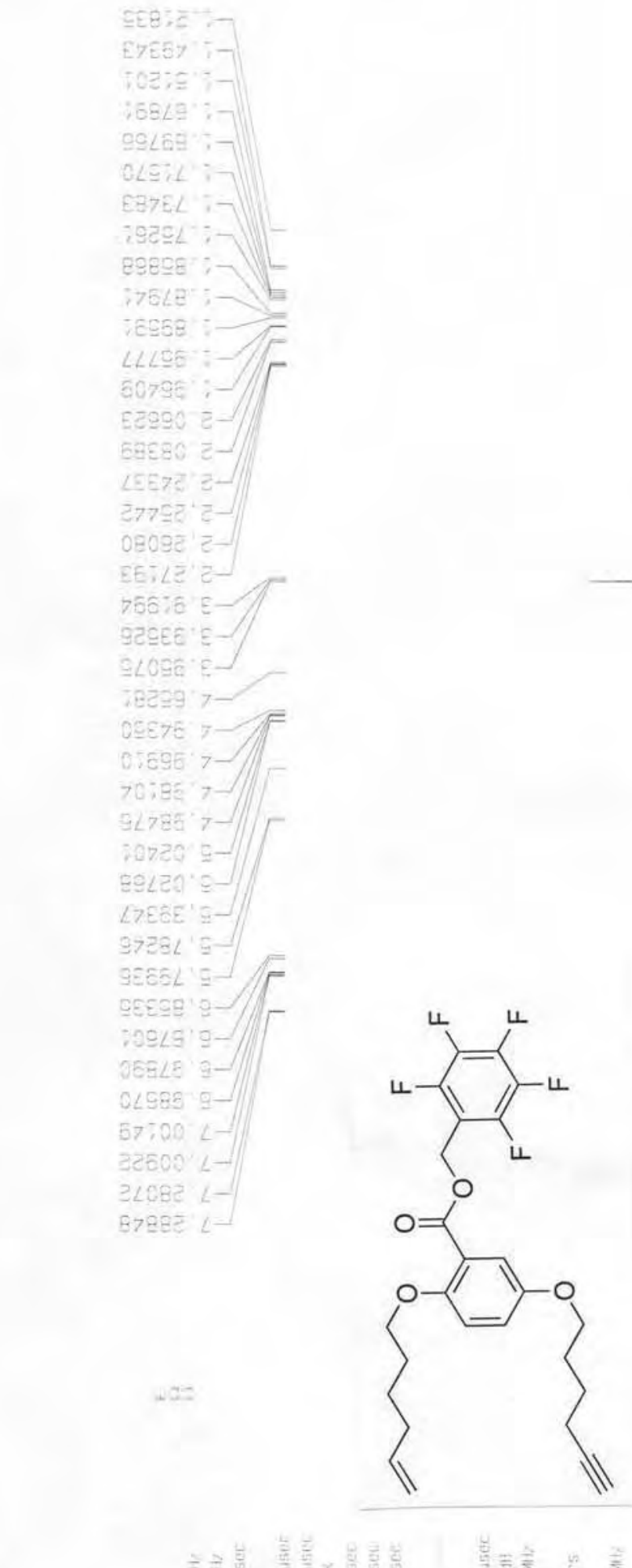

$\measuredangle$

ल)

$-x$

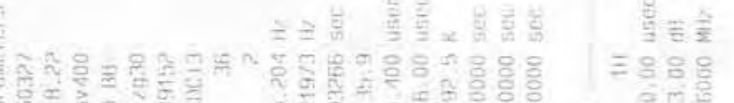

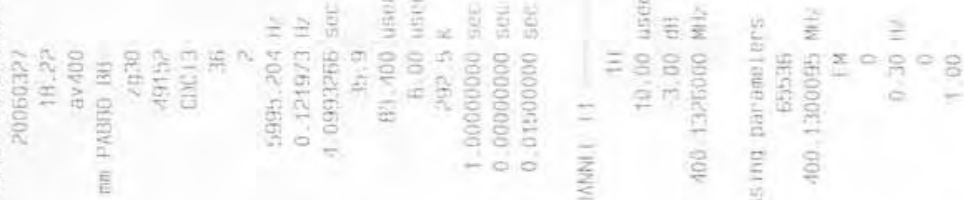

$\frac{5}{x}$

E.

Q

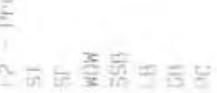
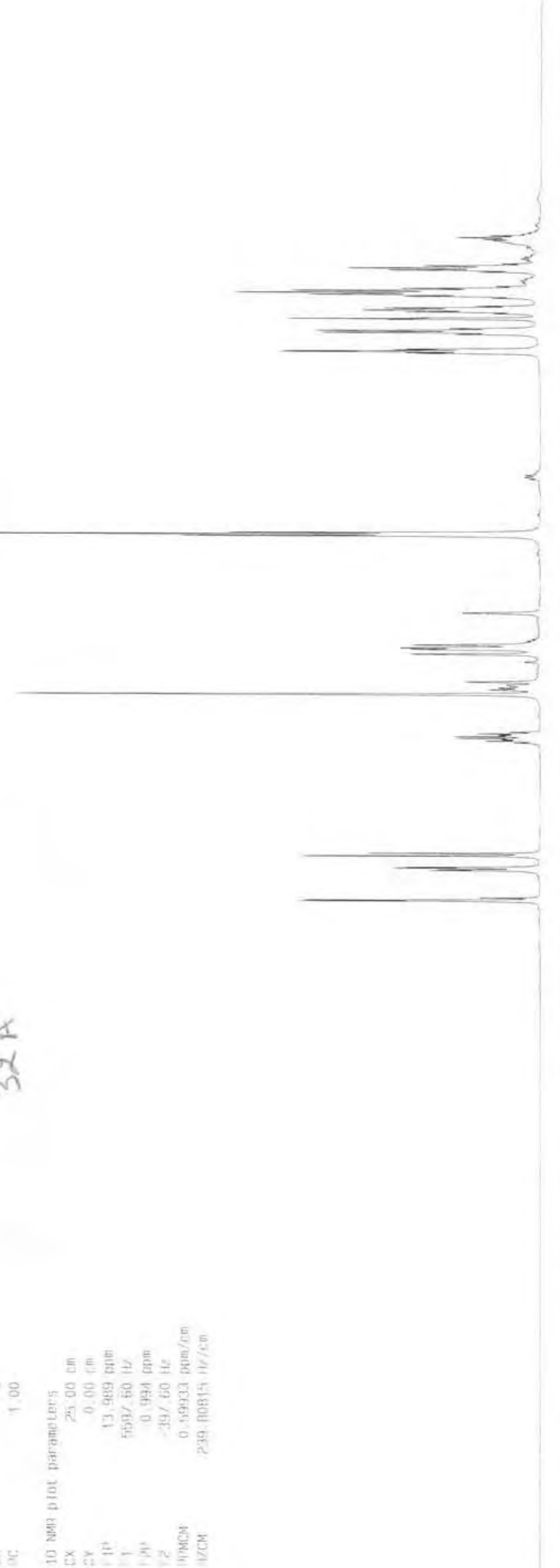


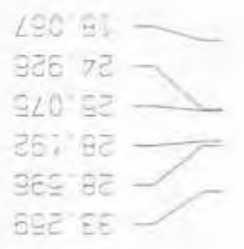

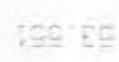

$905^{\circ} \mathrm{L9}$

960199

$\angle 0759$

789 9L

$7.00^{\prime} \mathrm{LL}$

कiE LL

E96" 29
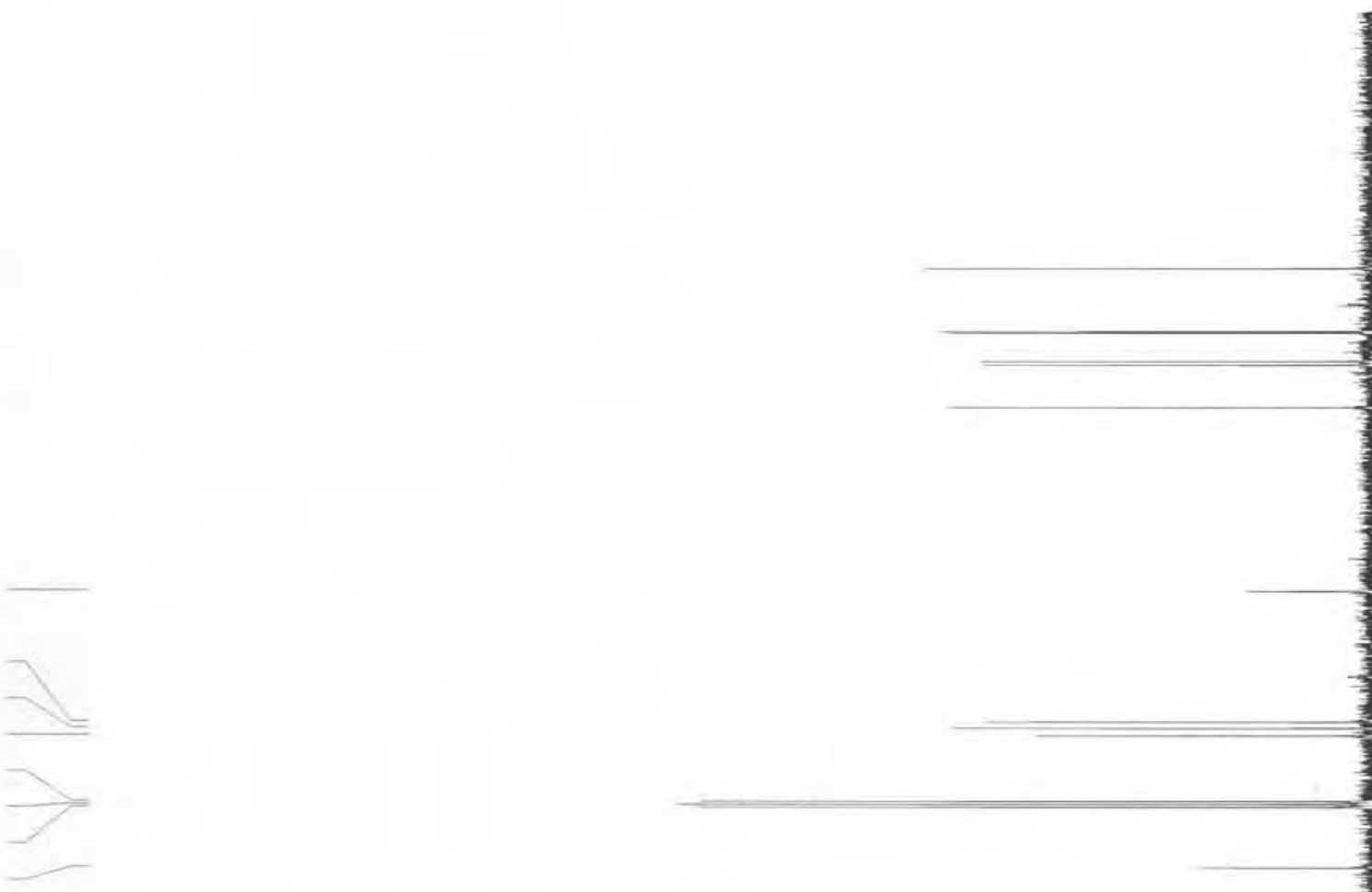

$969^{\prime} 60$ :

909 '

巨海 $7:$

ป9L'อ7:

zeg'⿺⿻一𠃋十

LSE QE:

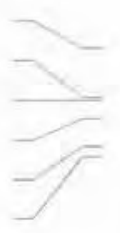

GLE BEI

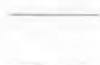

उE7 77 ;

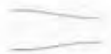

cos 37

:

690' हE?

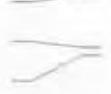

ctý

Lat
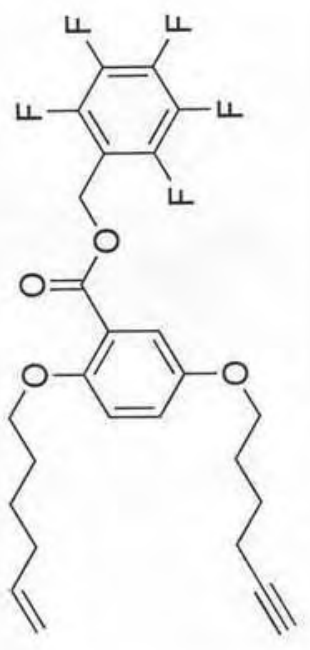

$\varangle$

o

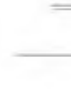



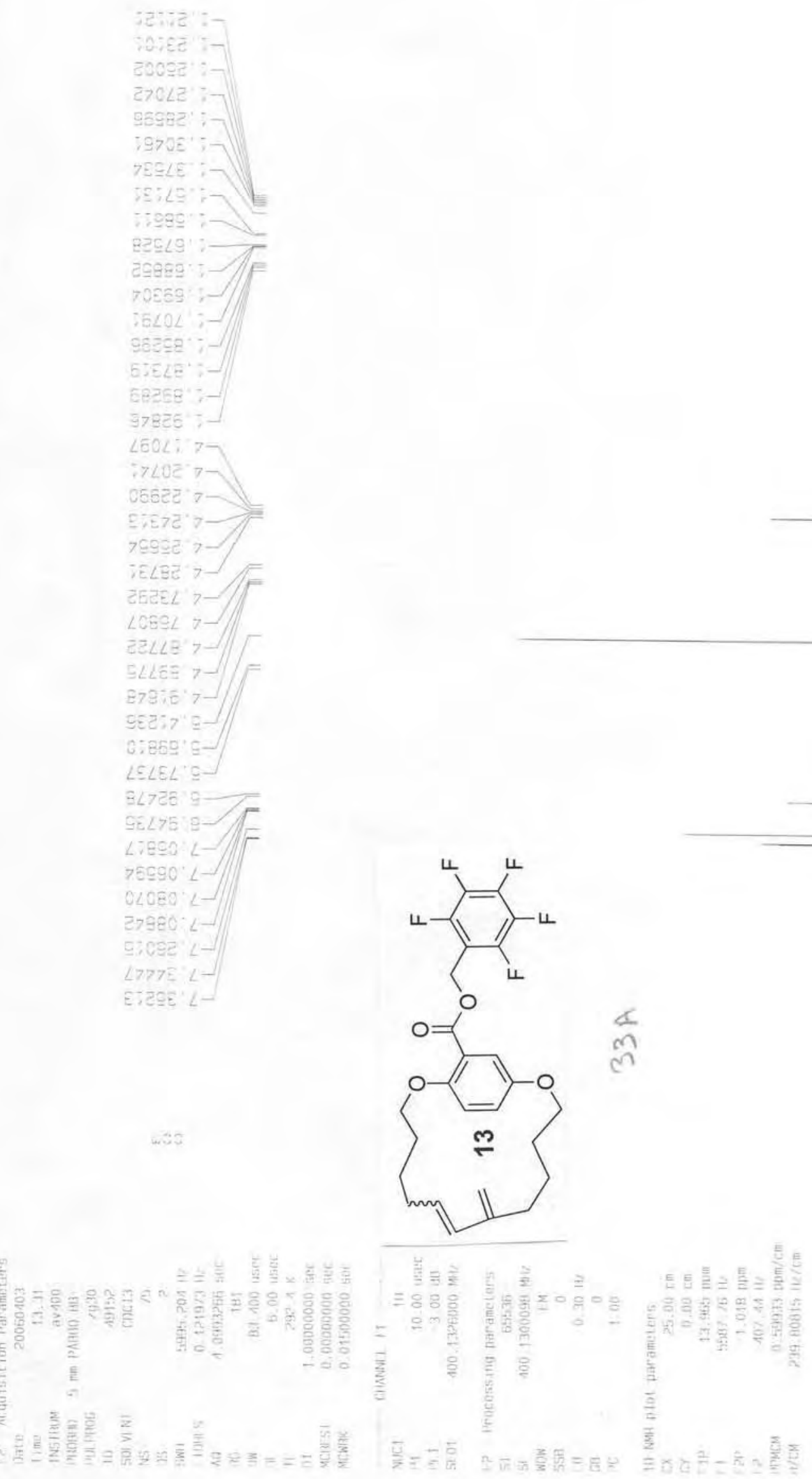


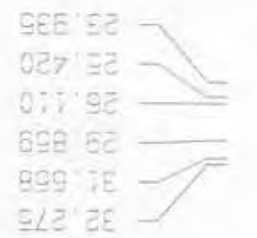

$99 L^{\prime} 69$

5E9 89

$\angle B L 59$

IB9 $9 L$

68692

S:E 'LL
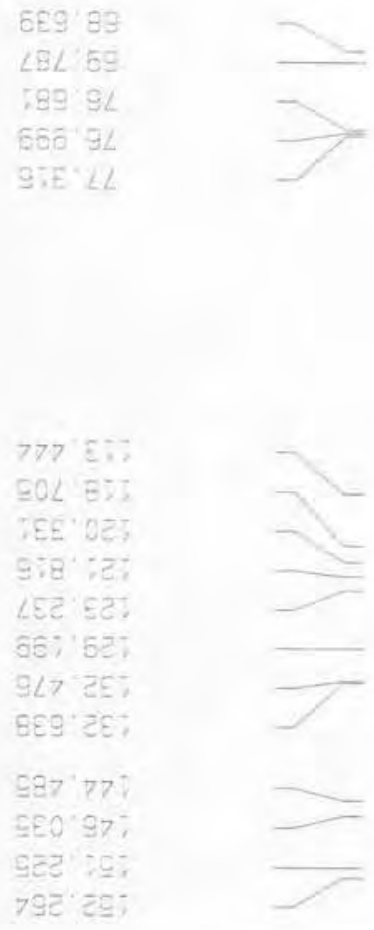

cLo ges

wad
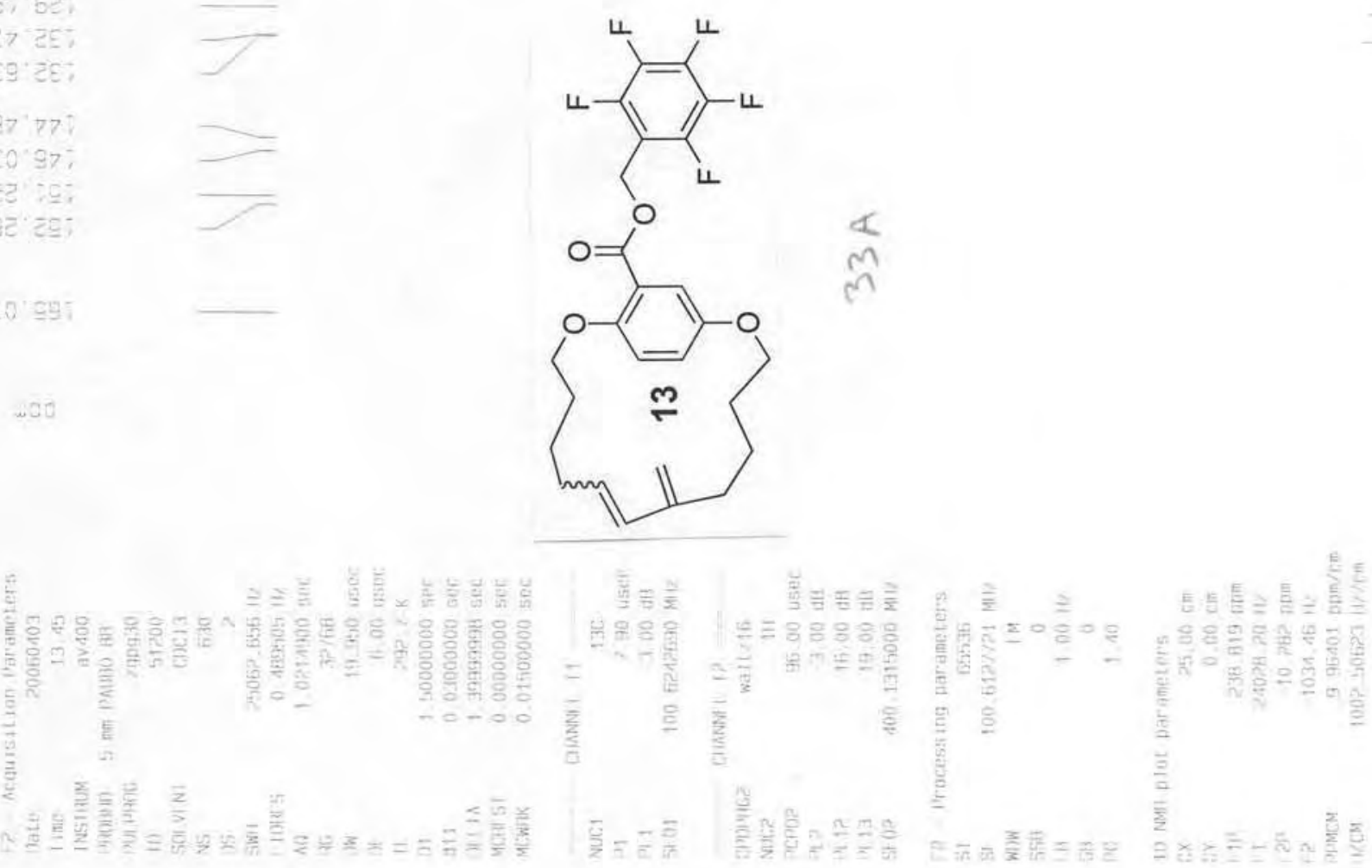

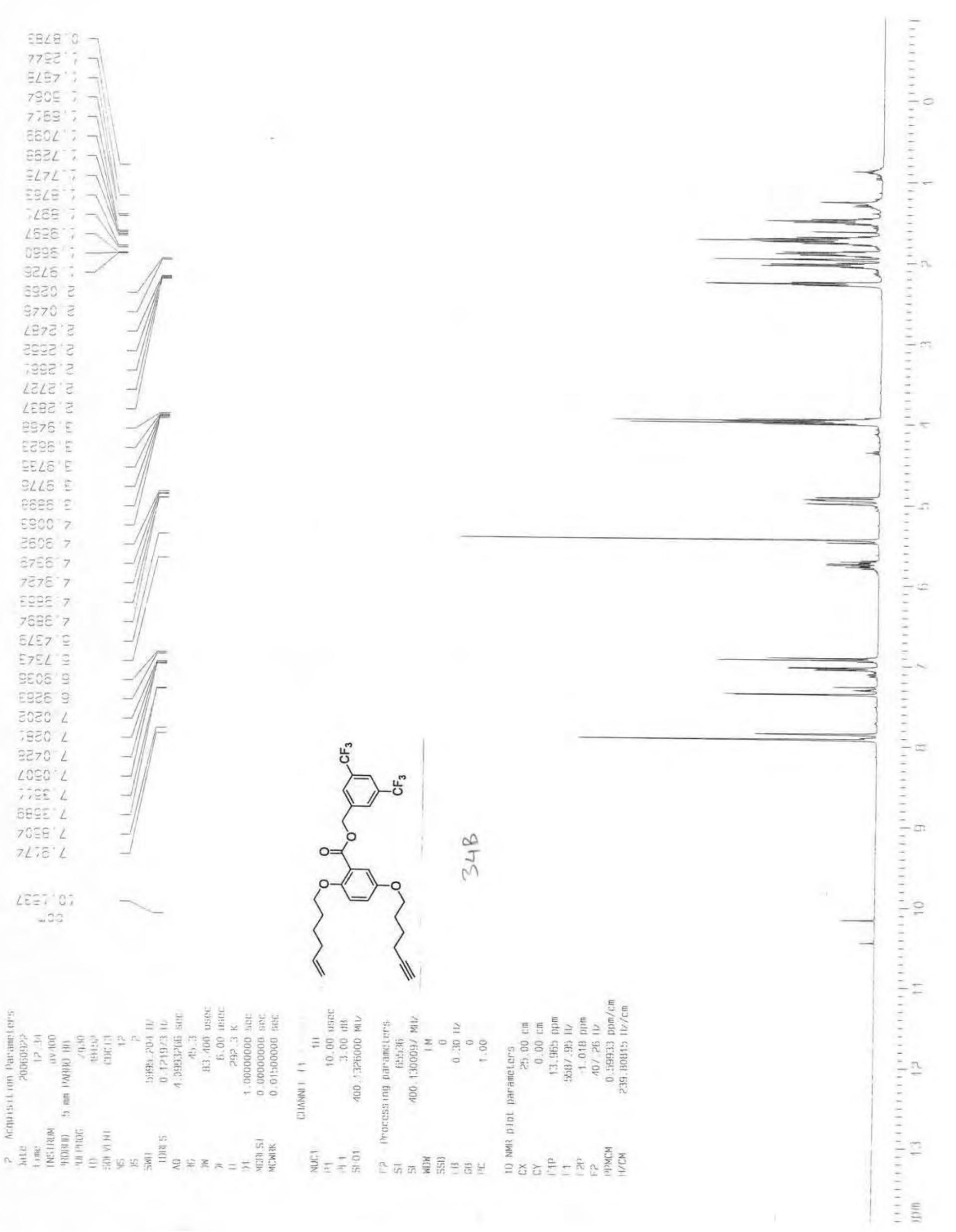

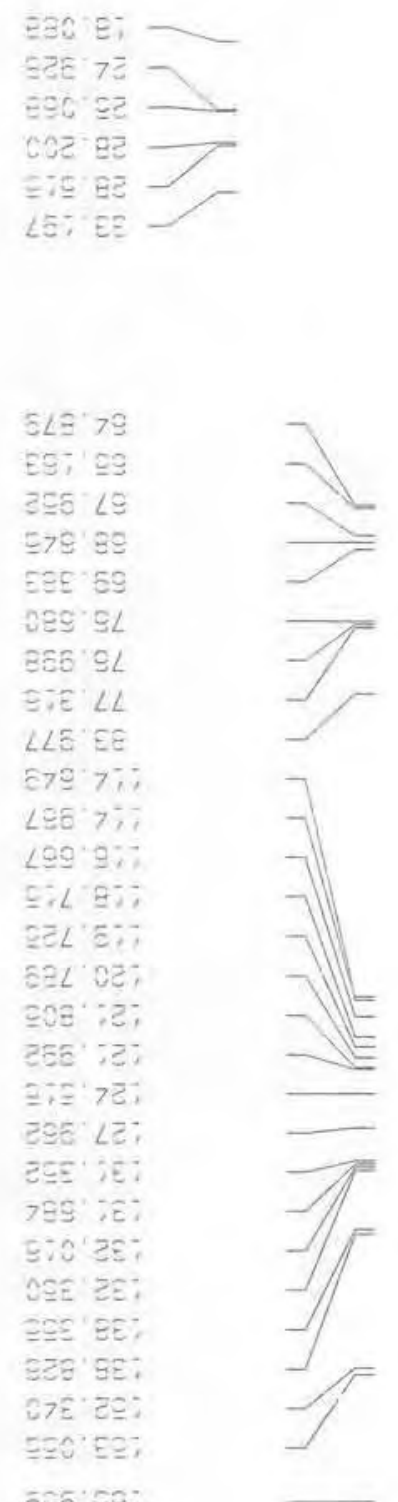

วะอ่อะ:

was
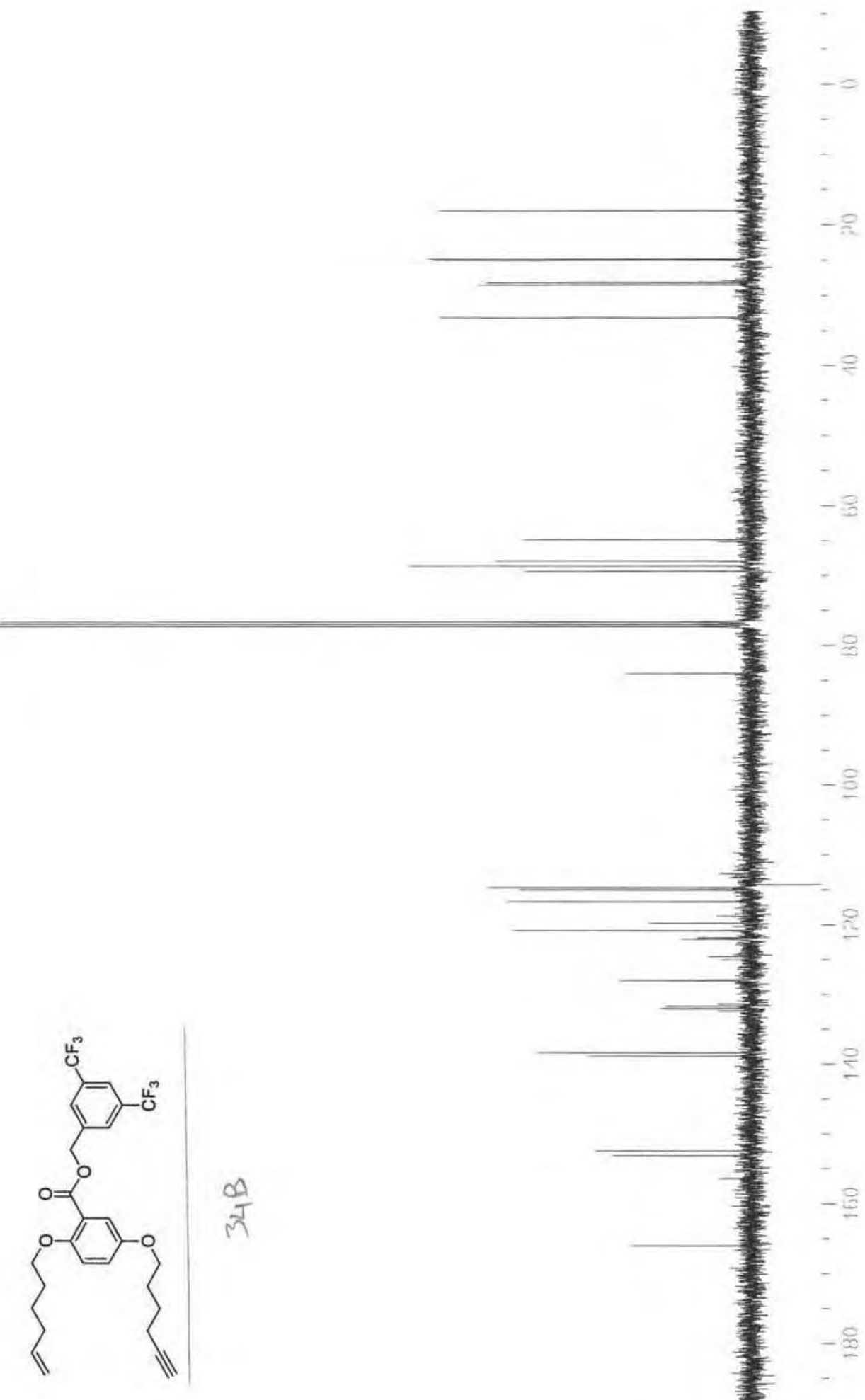

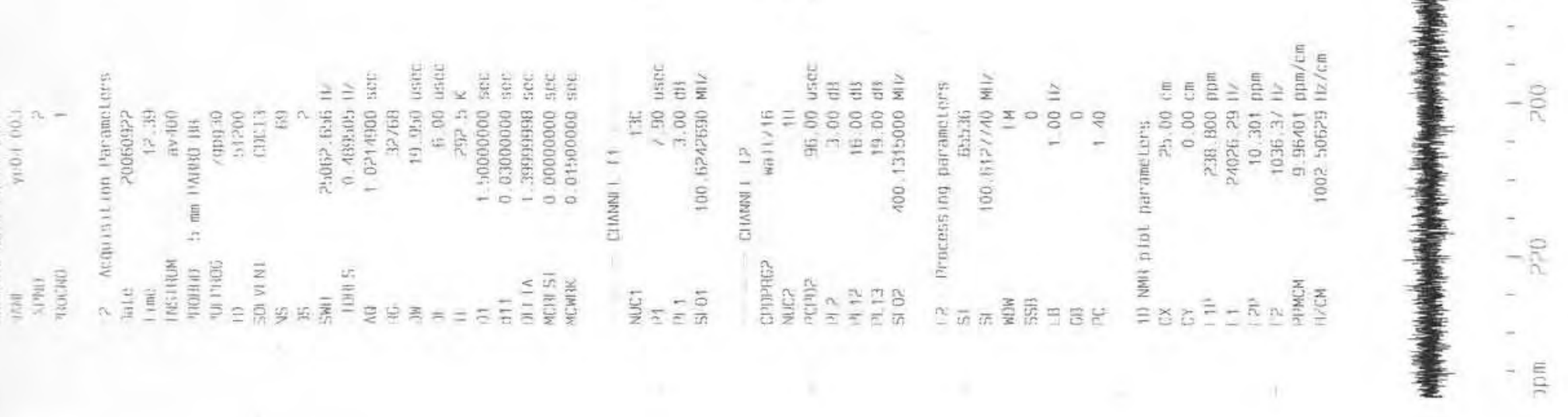




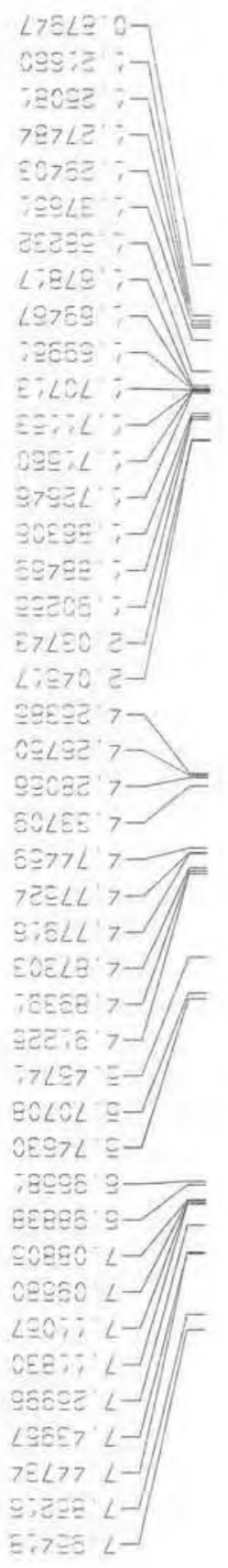

$-25$

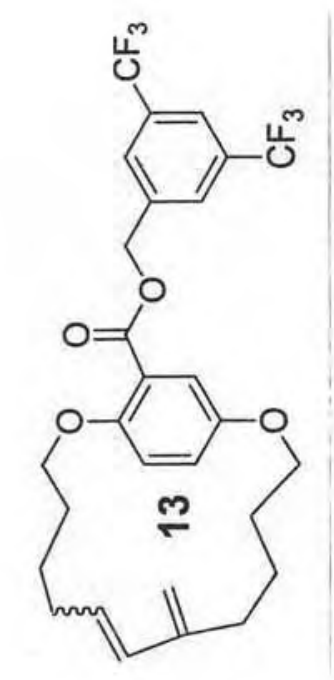

की

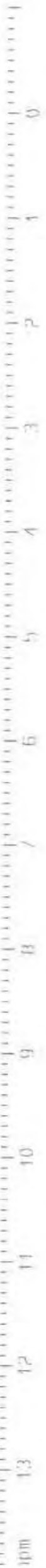




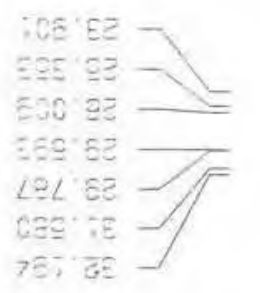

Tละ 79

$i \in 799$

ELL 59

gES $9 L$

EOS LL

उEE LL

LEQE:

ZLE B::

हอ7 28 ;

¿75 洨:

हiอ iz:

टอร हE;

CSe 7 :

इ⿻日 $L Z$ :

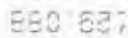

Bze ie?

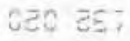

ऽLミこE;

SIE QE:

ऽอ6 §7;

도: is:

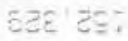

$\Xi, L \subseteq:$

$-32$
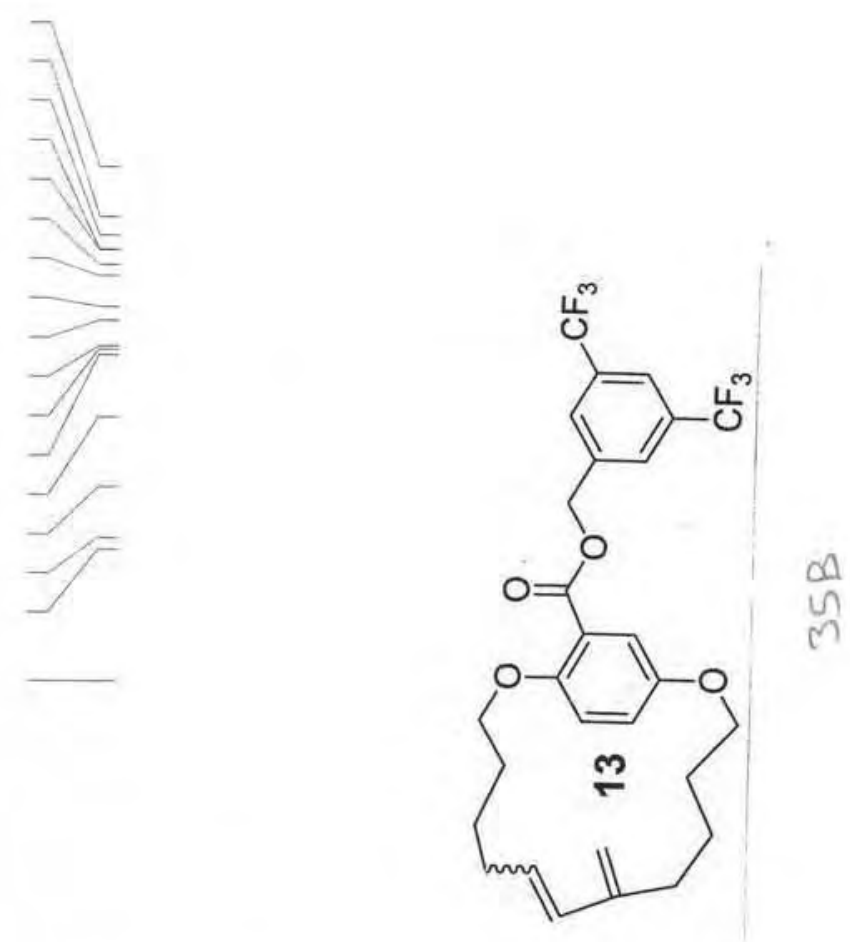

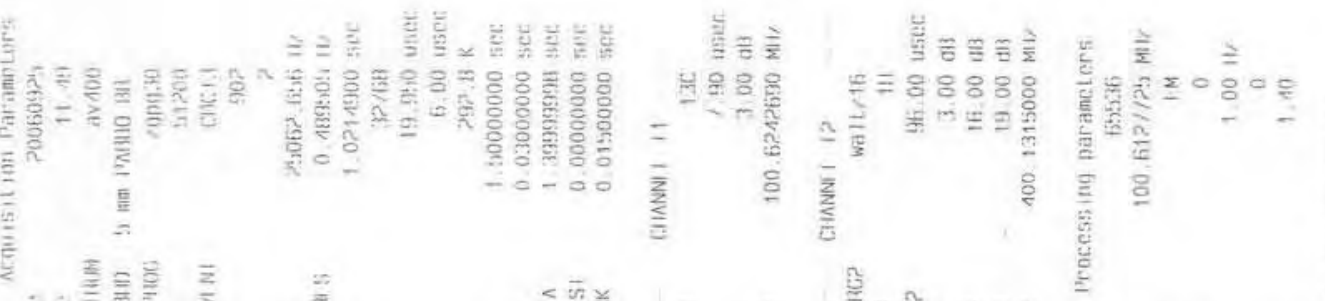

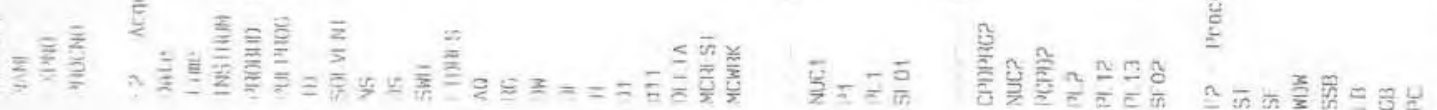

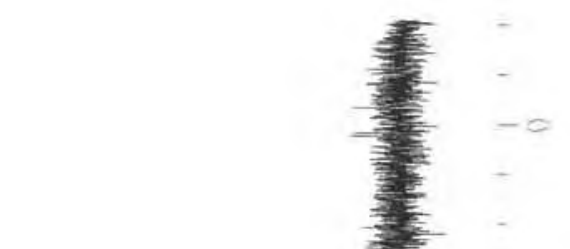




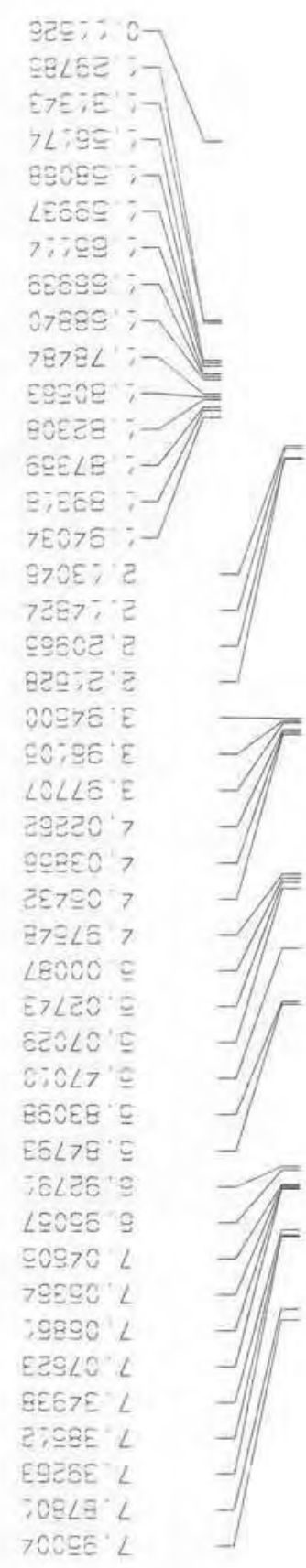

ב

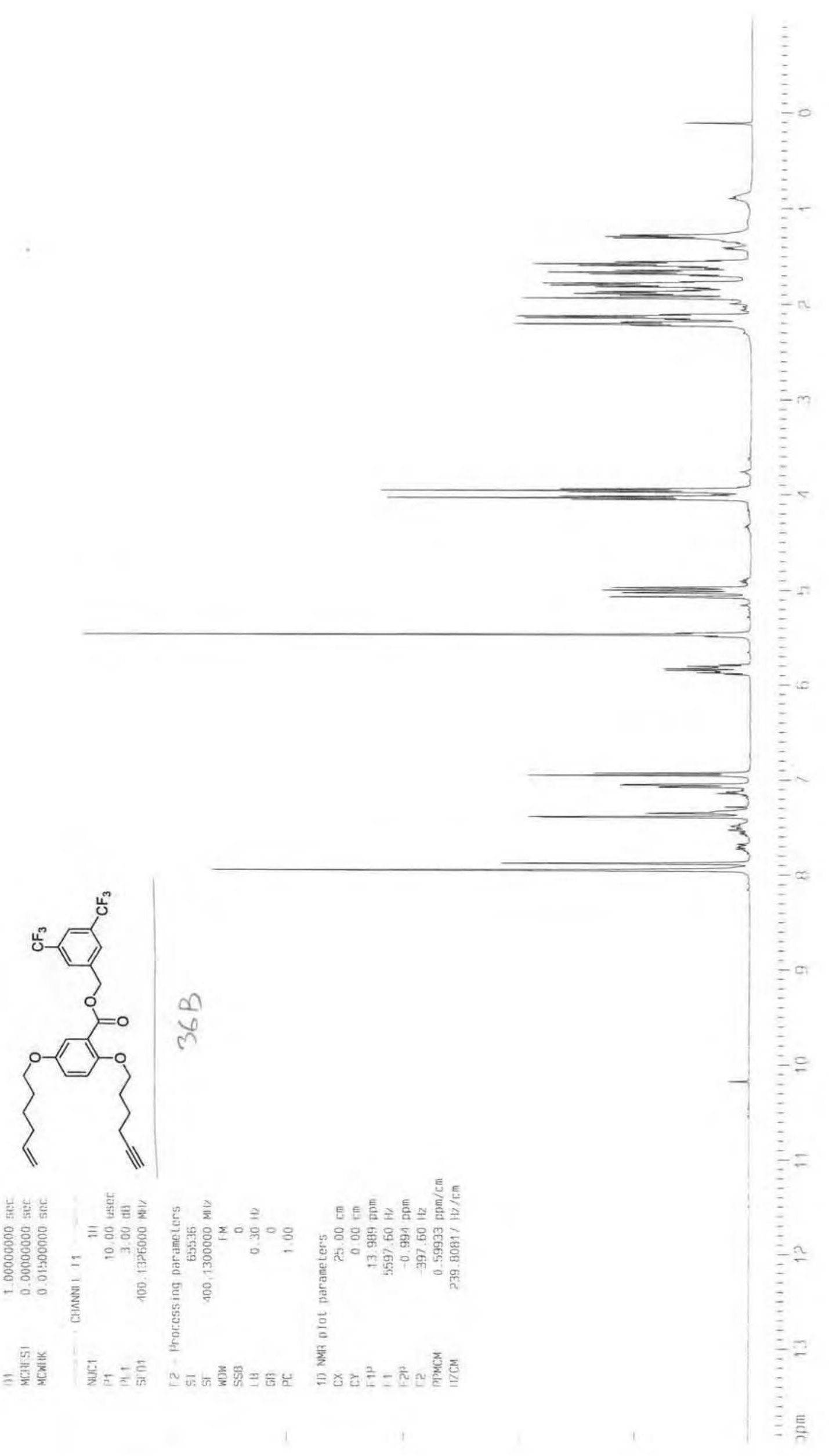



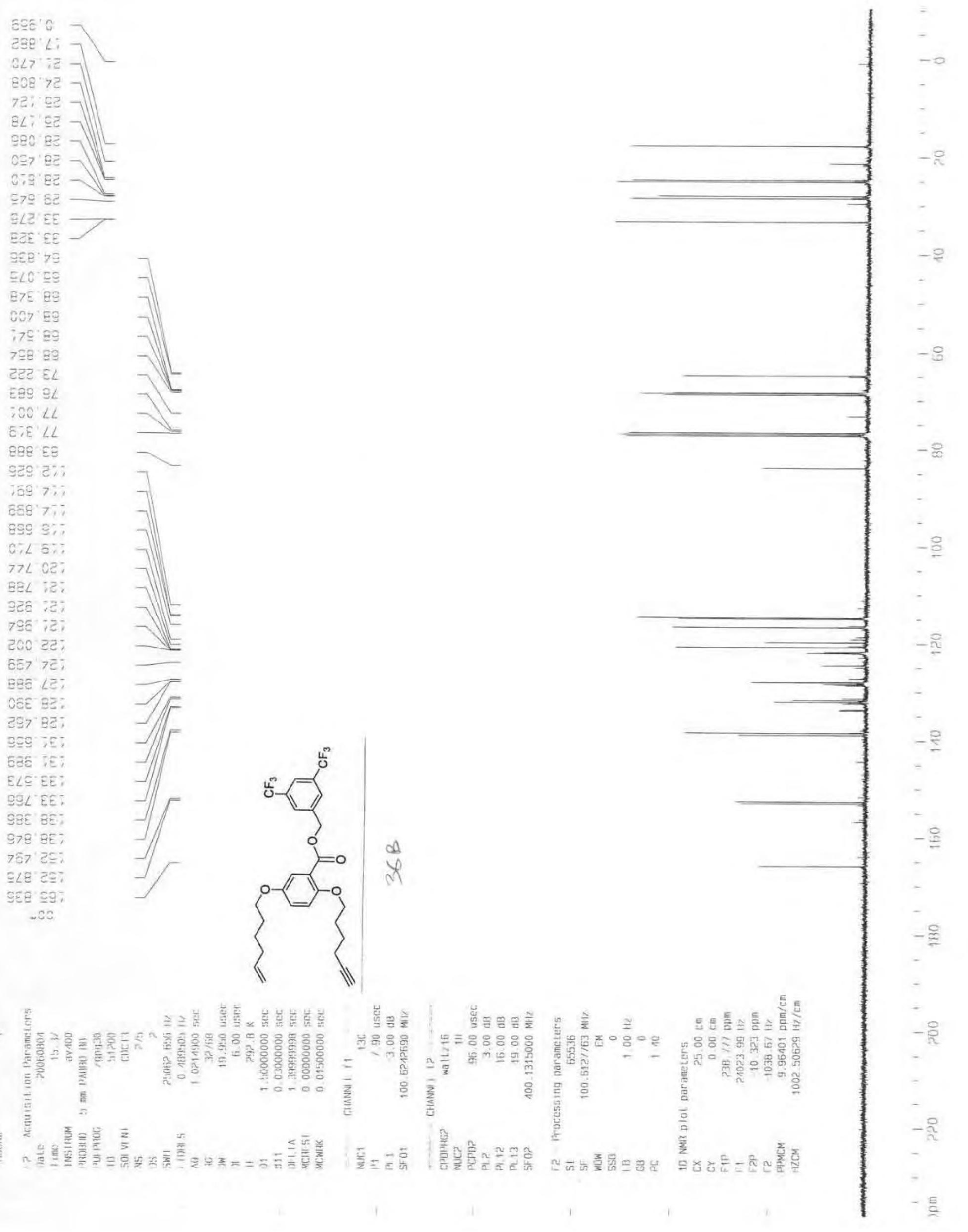

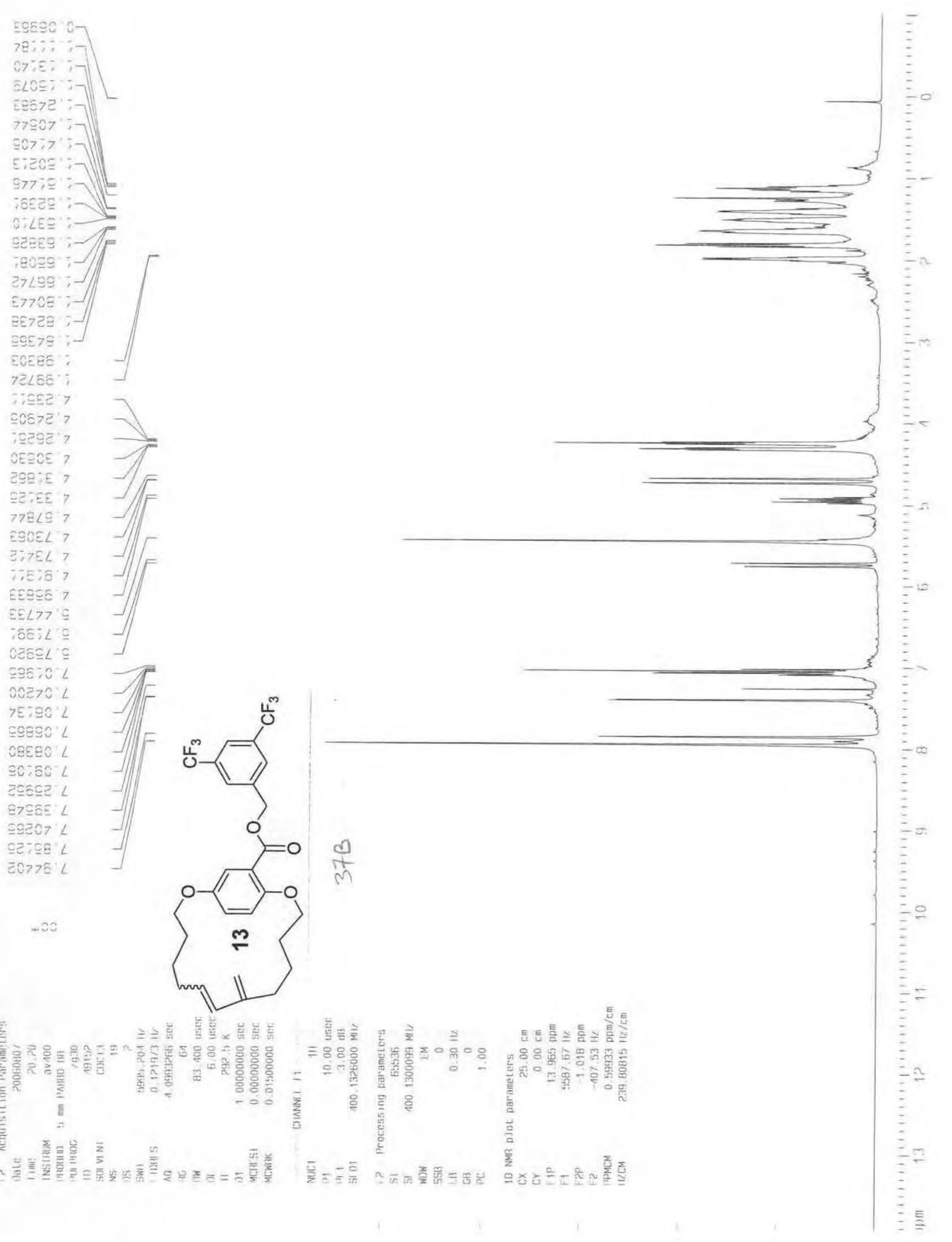

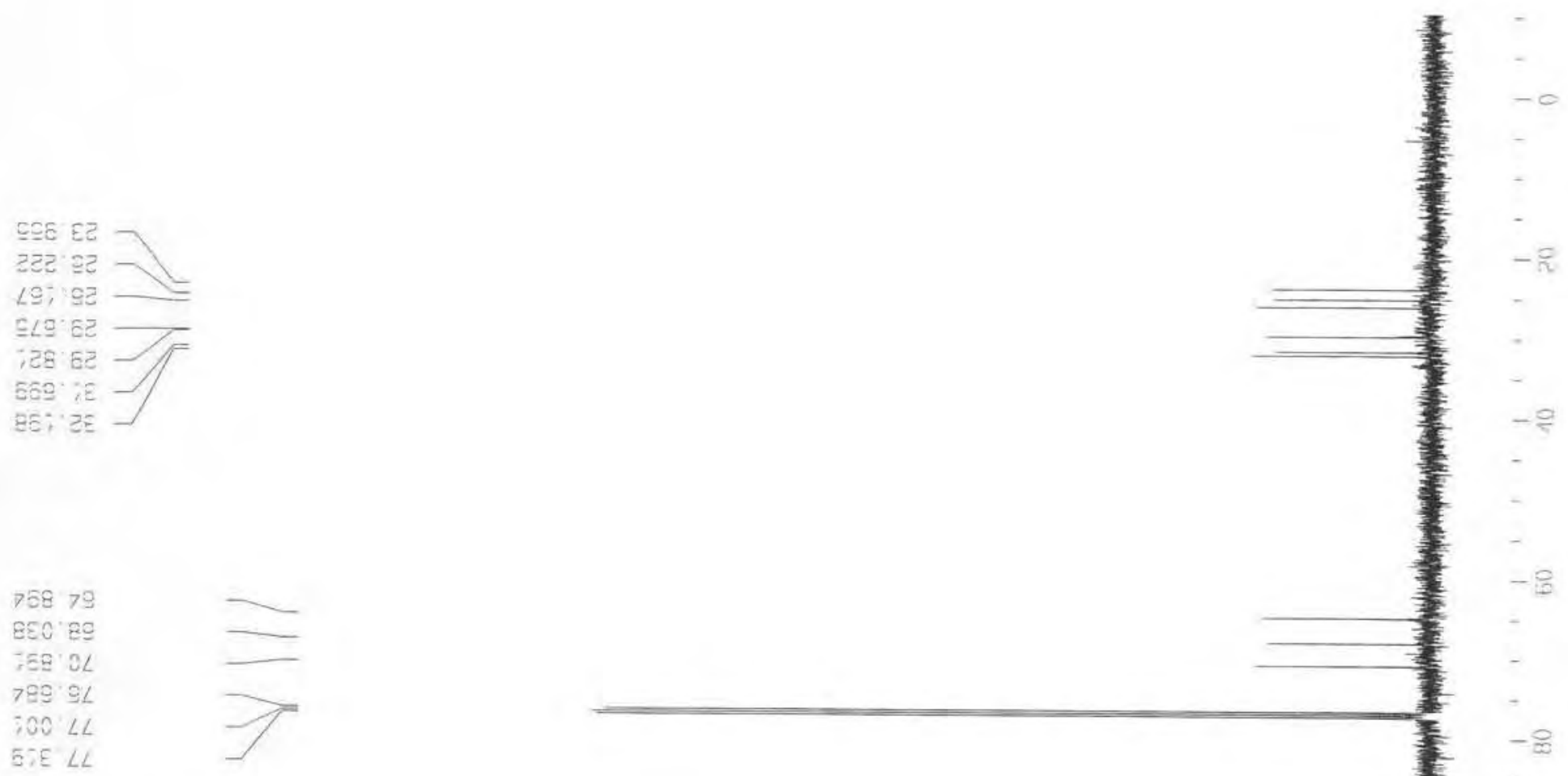

E泟E:

क्ट० हा:

व:E 0己?

LउG' iz:

ฮ己; ここ;

8E> E?;

ตEะ 72 :

$7 E L L E$ :

LEO EZ;

Ees ie;

टडर $25:$

BOS टE:

6.8 8 ;

; $8 \mathrm{9} \cong 7$.

घटc 0

$0 \equiv 0$ हE;

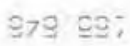

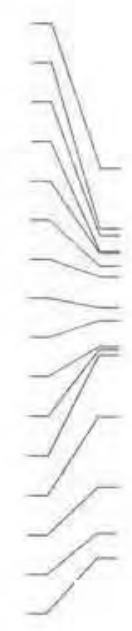

. .50

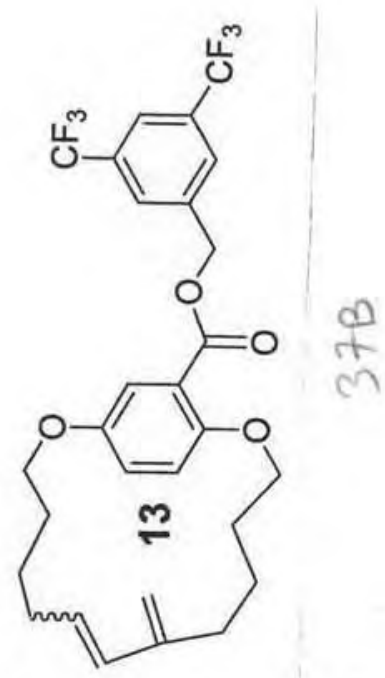

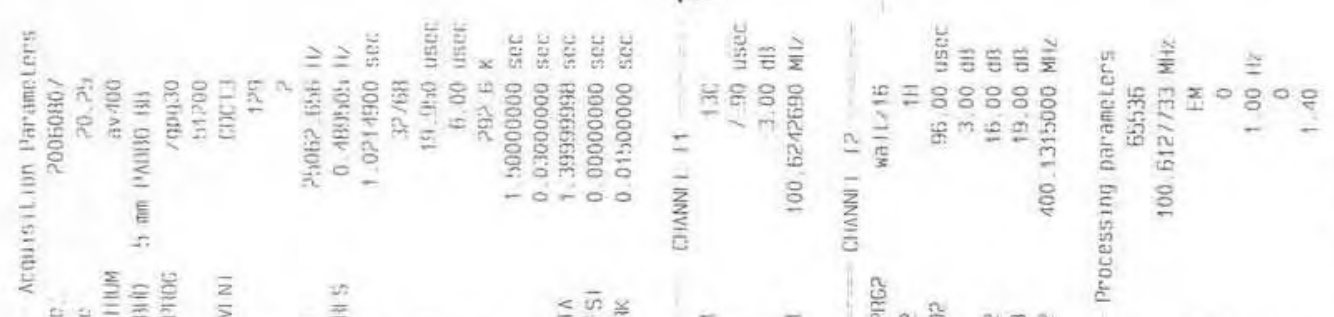

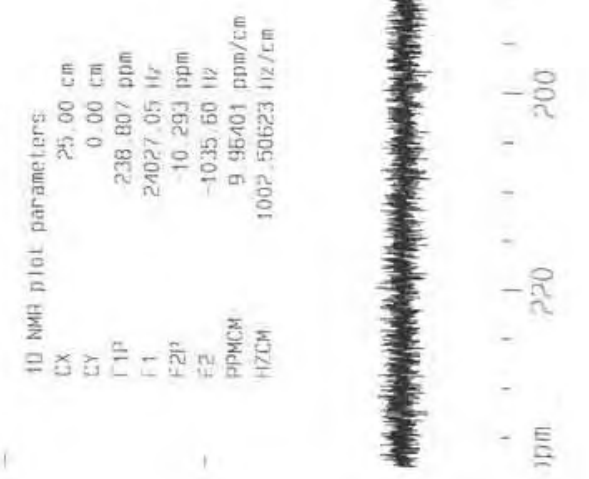




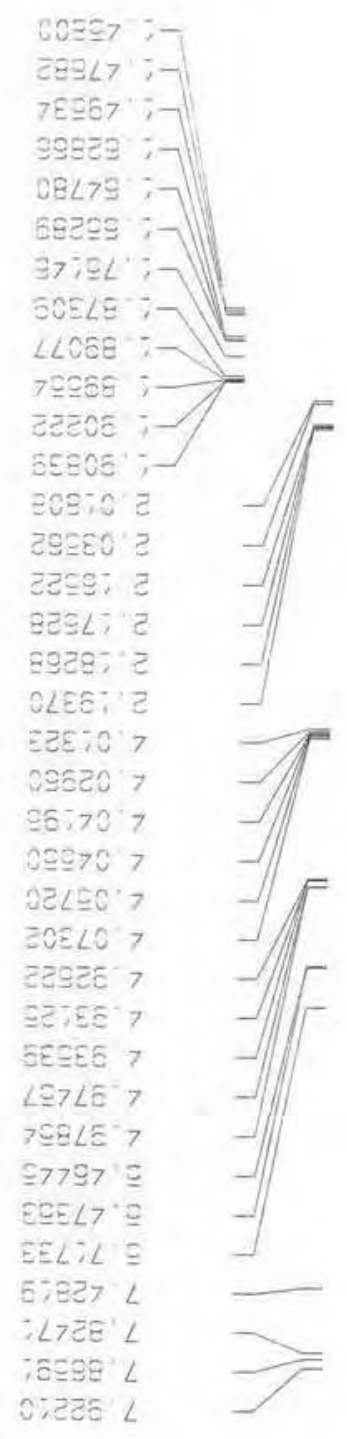

$-32$
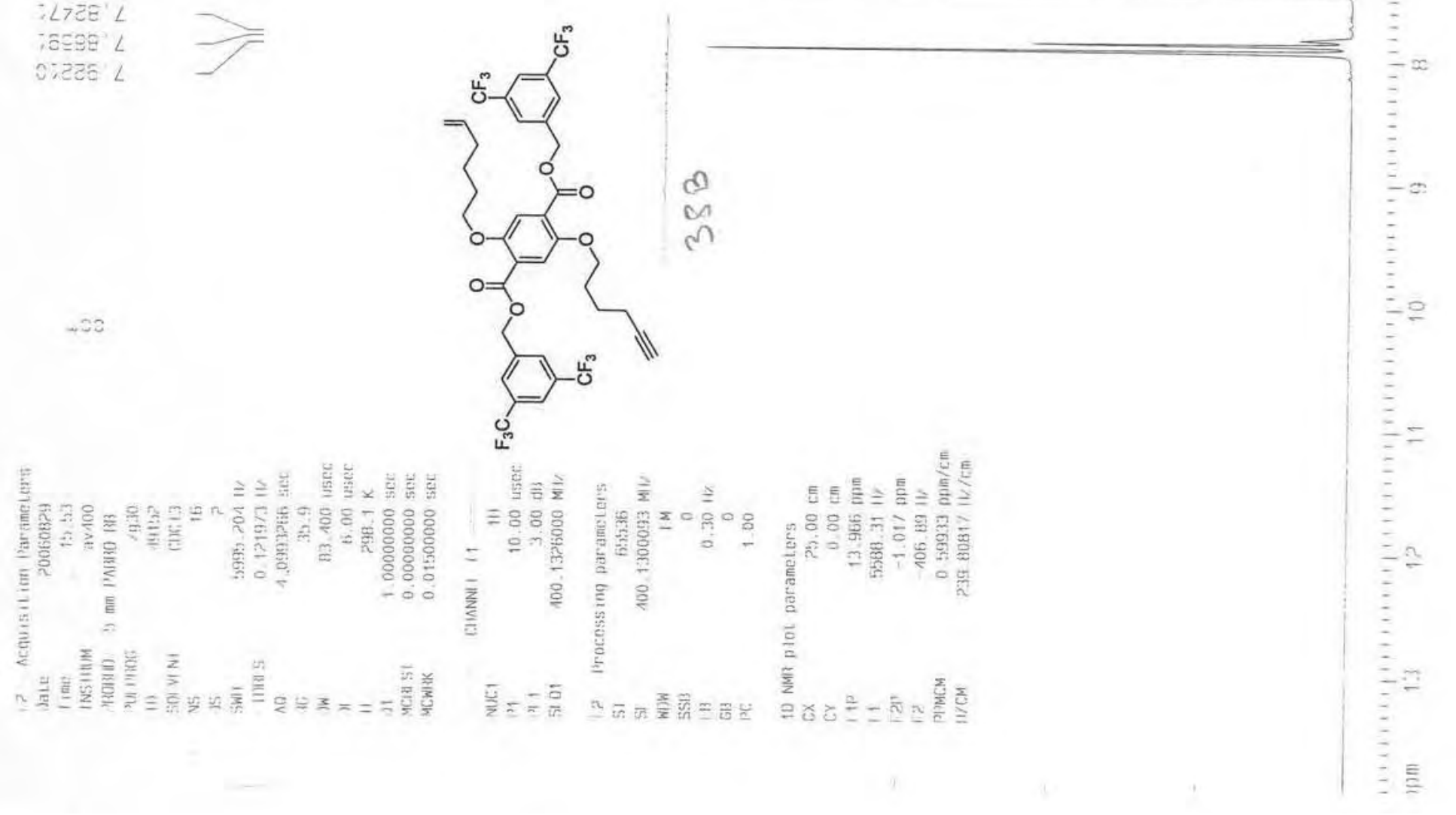

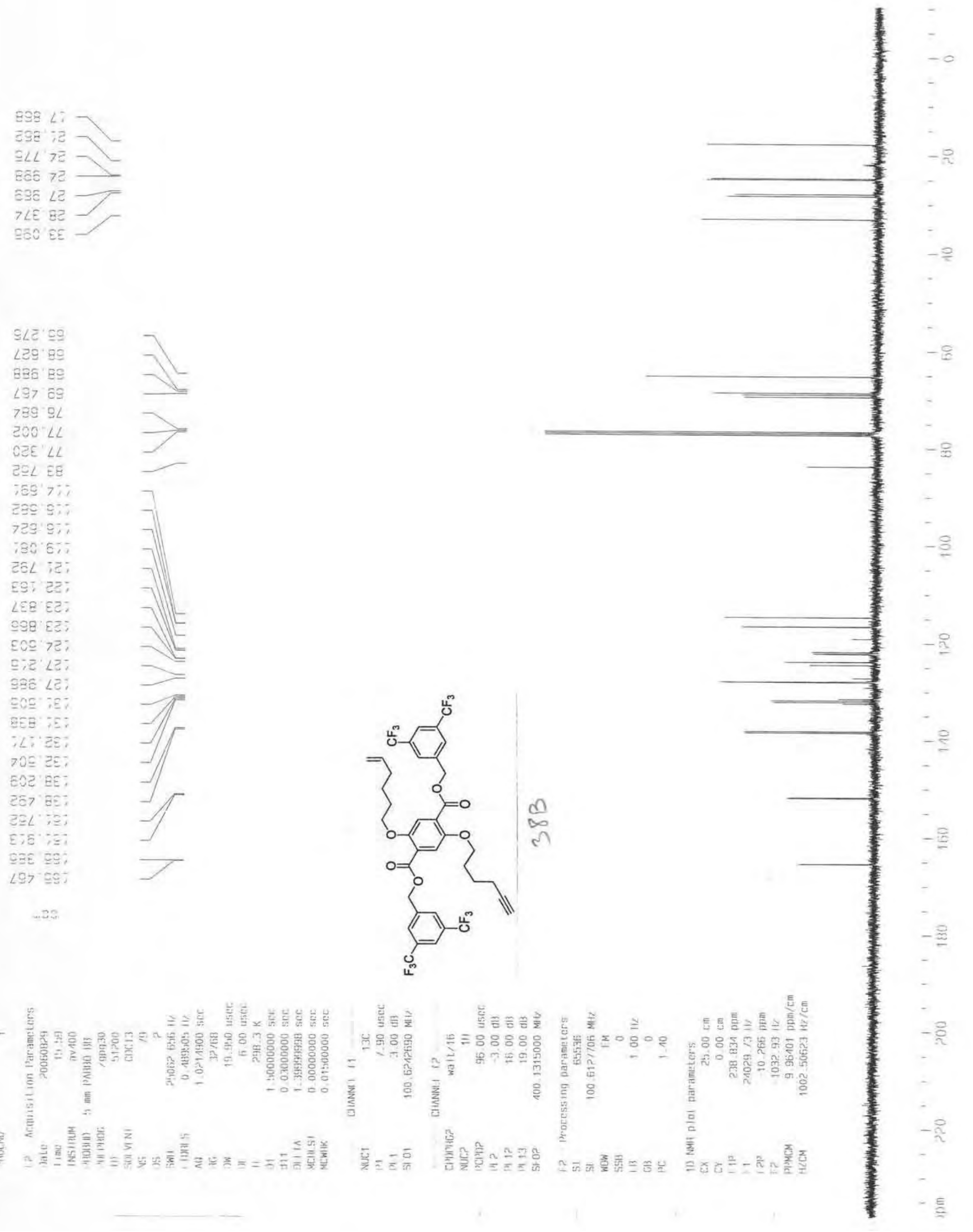

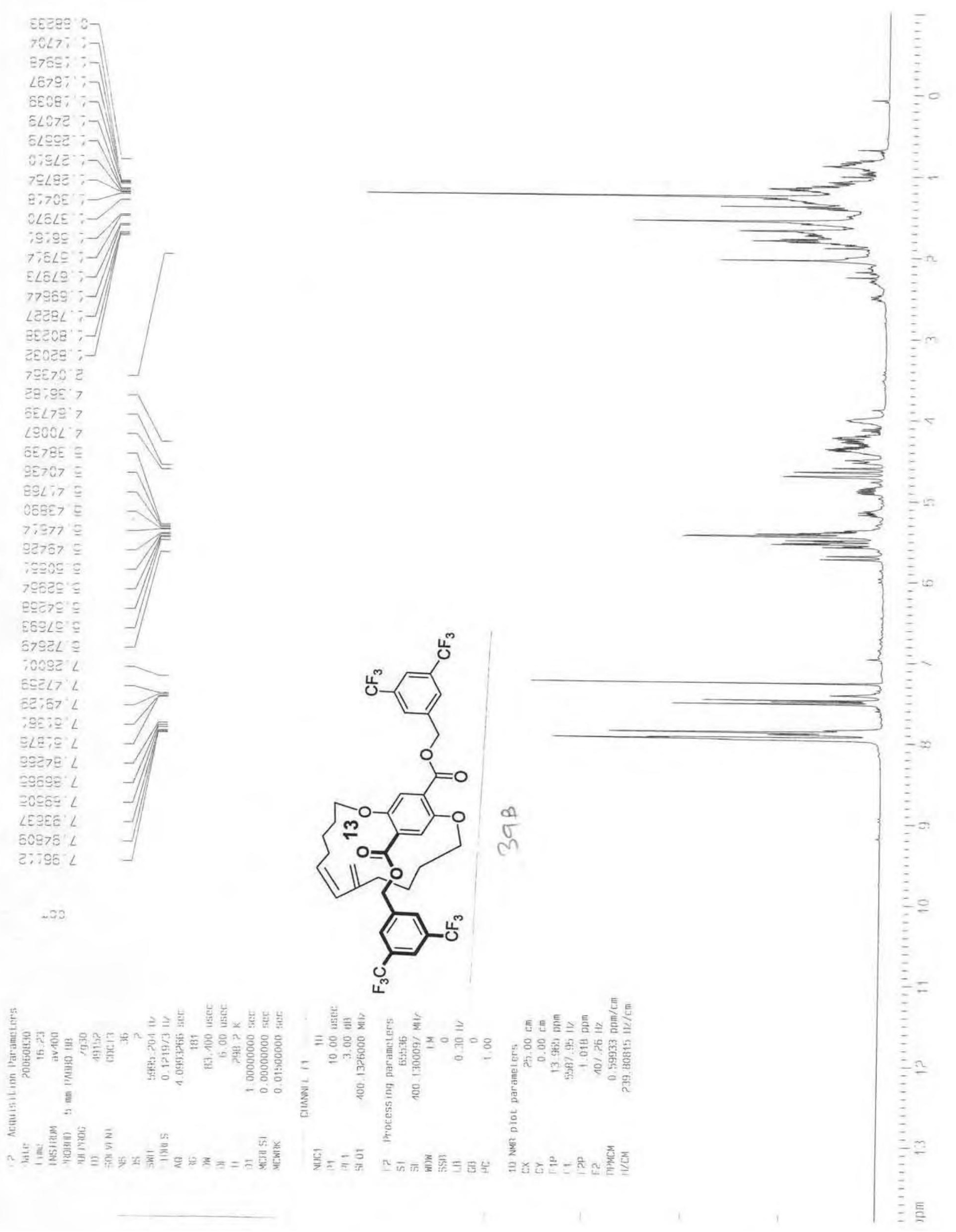

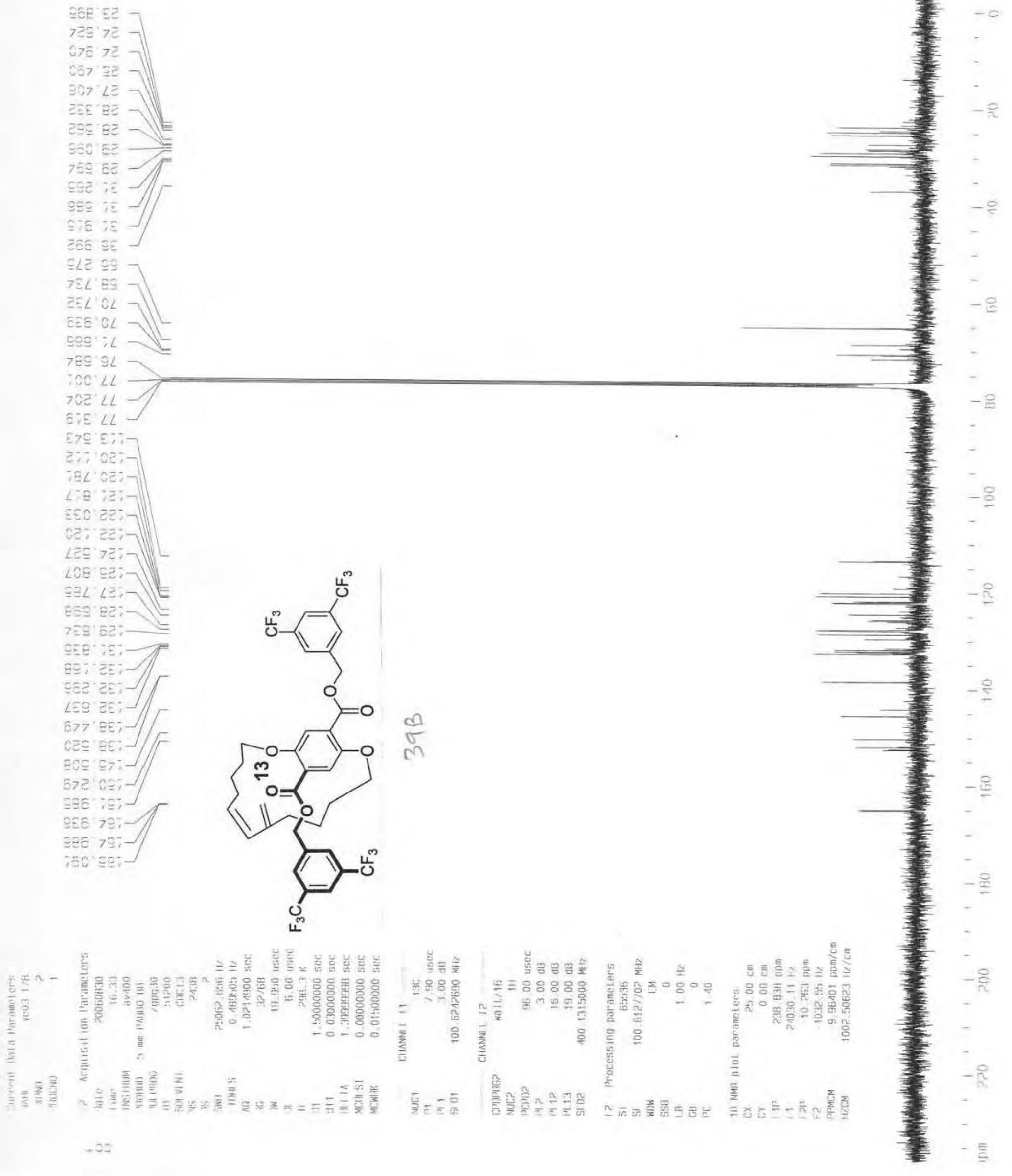


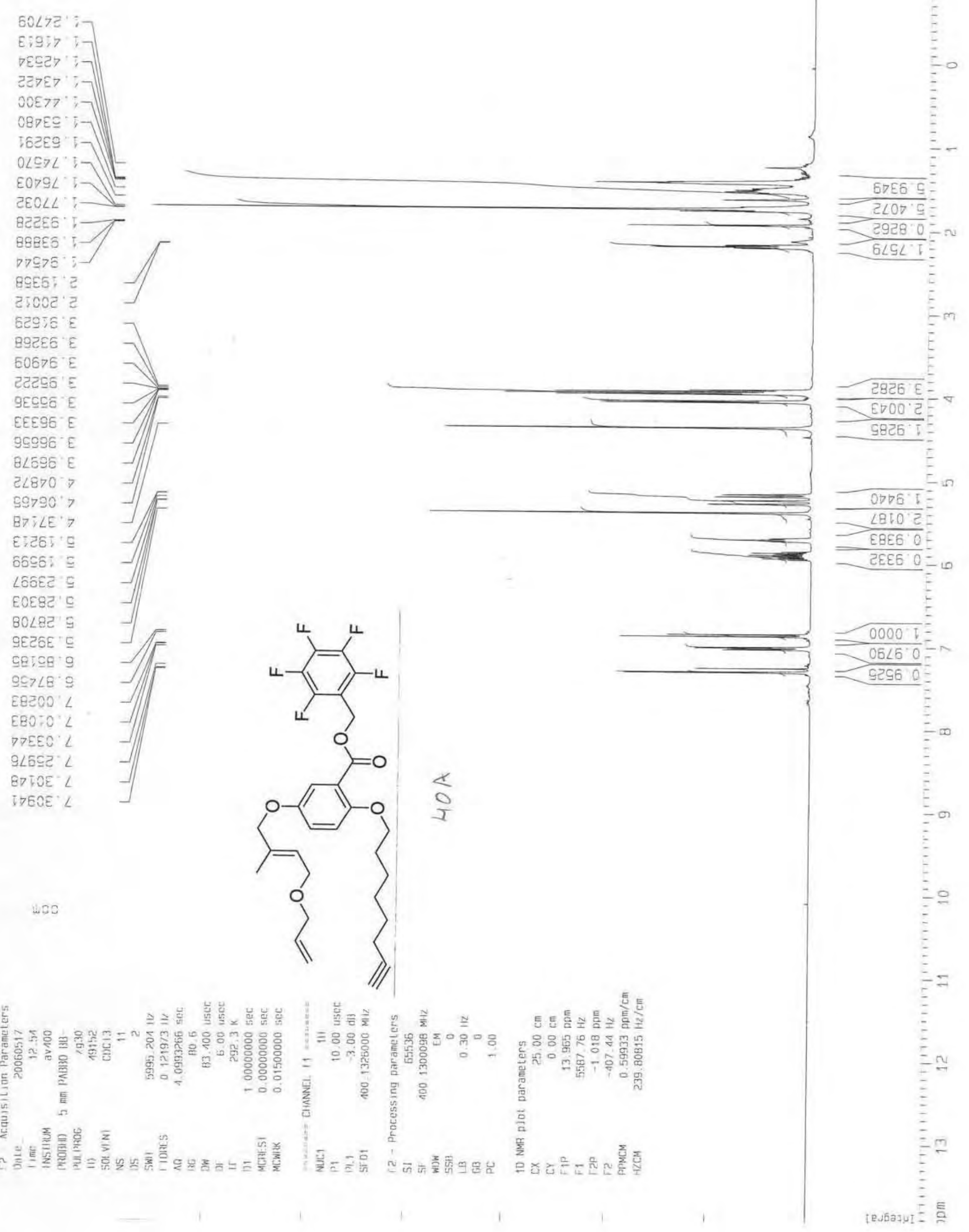




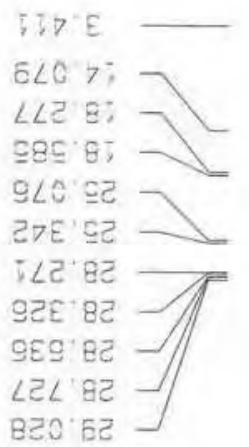

\section{BLS EC}

$\angle 66 \mathrm{CS}$

ZL; $B 9$

Bะ7 ธ9

BST IL

GES'EL

TEQ $9 L$

666.94

9TE LL

$\varepsilon \angle 7>B$
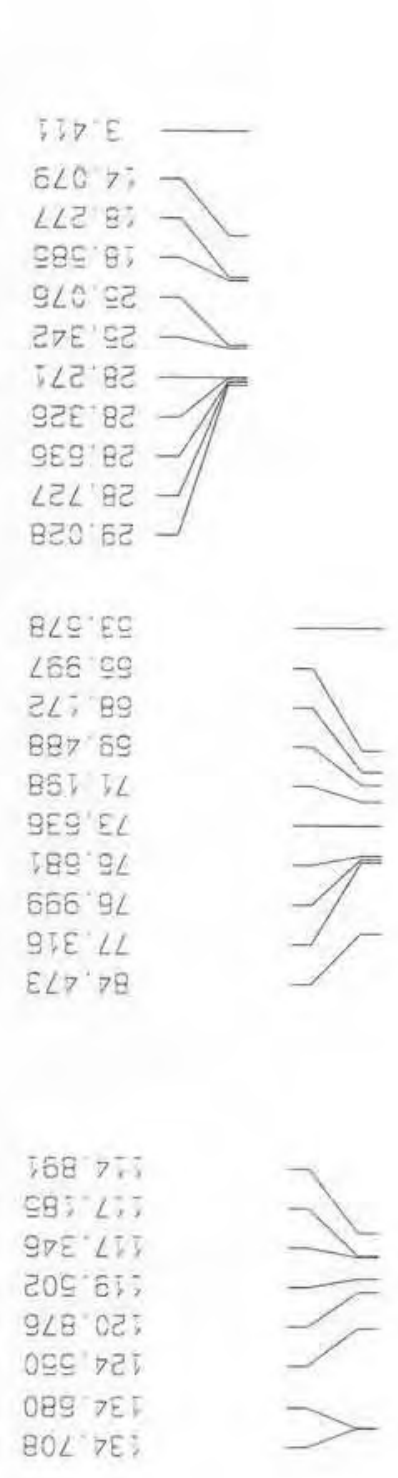

อIอ TE;

EEZ'EES

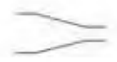

$7 \angle E$ C9?

wdC
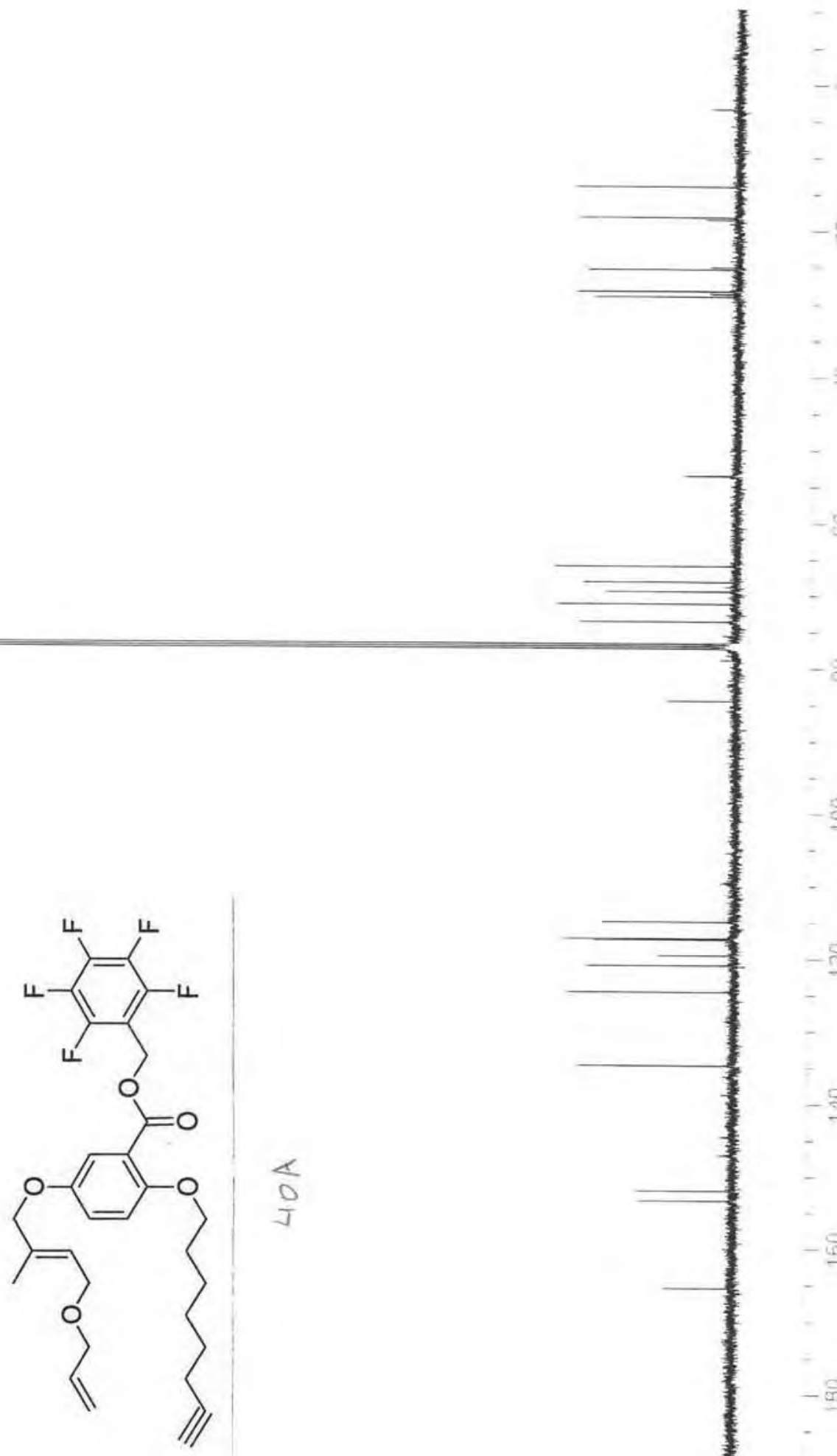

- 

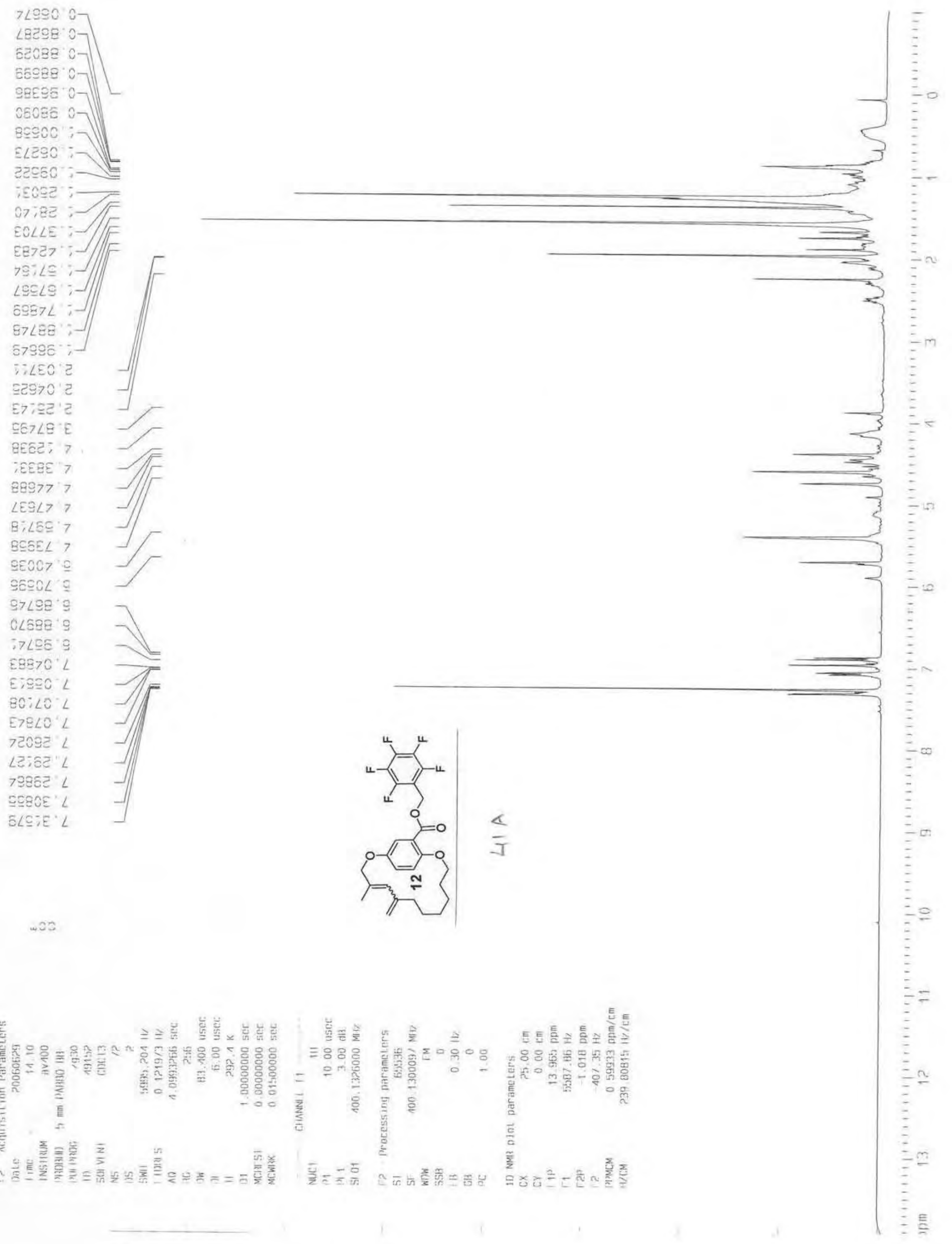


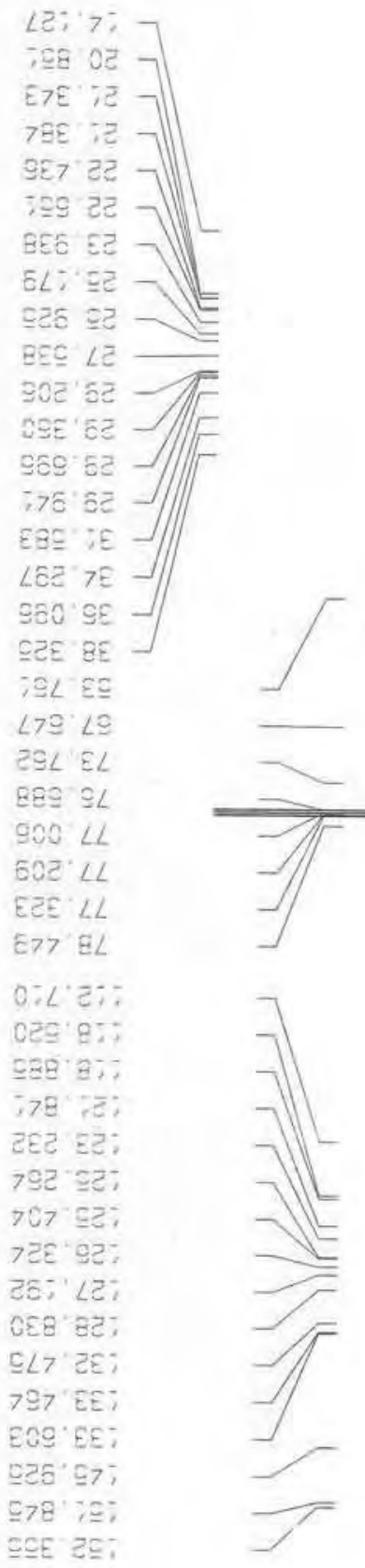

ELE'フ9:

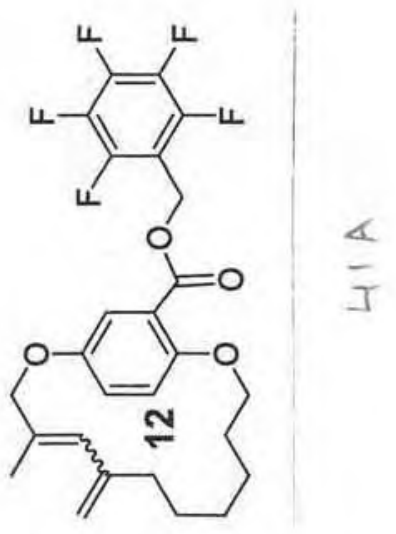

TCE 0

कL: ge

क्य

goc 3

7 ' के

马E iE

ce 78

टरF

ZSL EL

EEE 14

저 E

ESe 1

70 कर

707 G2:

CE. है ह

CL7 $\mathrm{Ze}$

$797 \mathrm{EE}$

EOS EE?

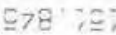

TSE 2 :

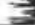

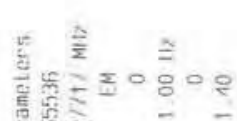

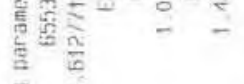
总豆

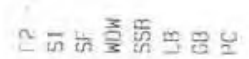

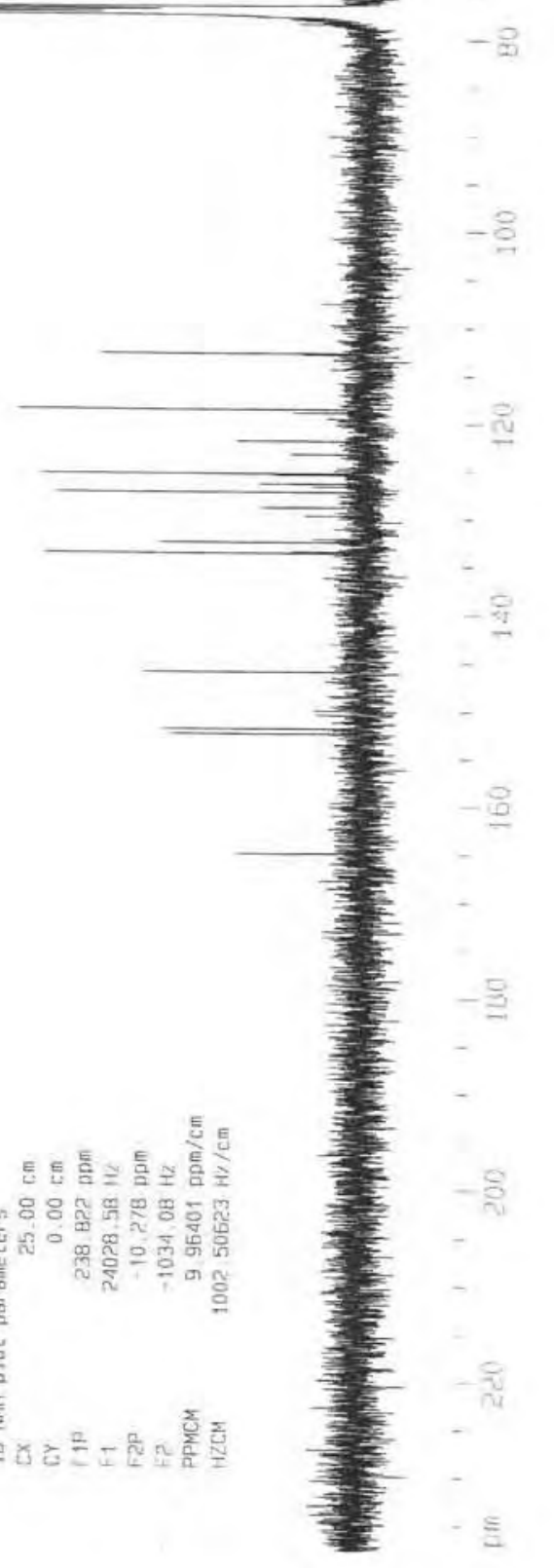




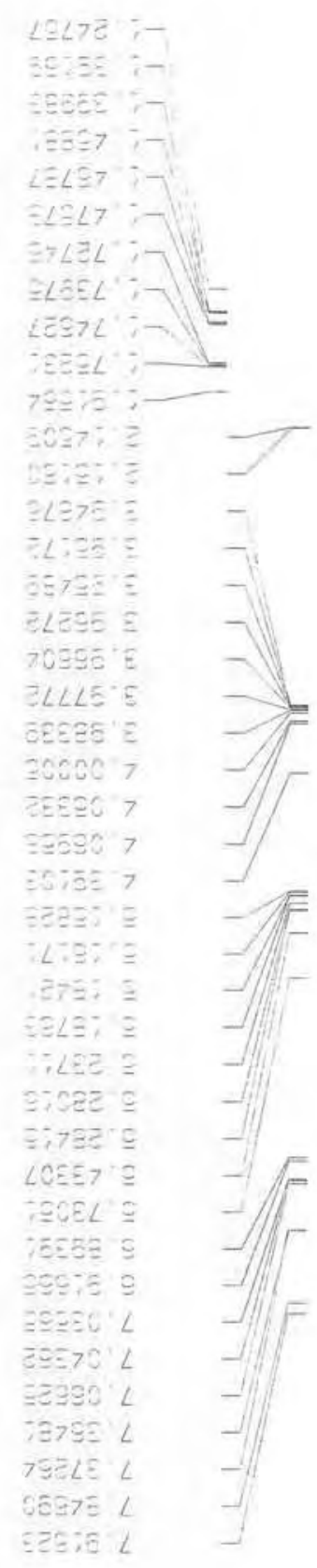

$-23$
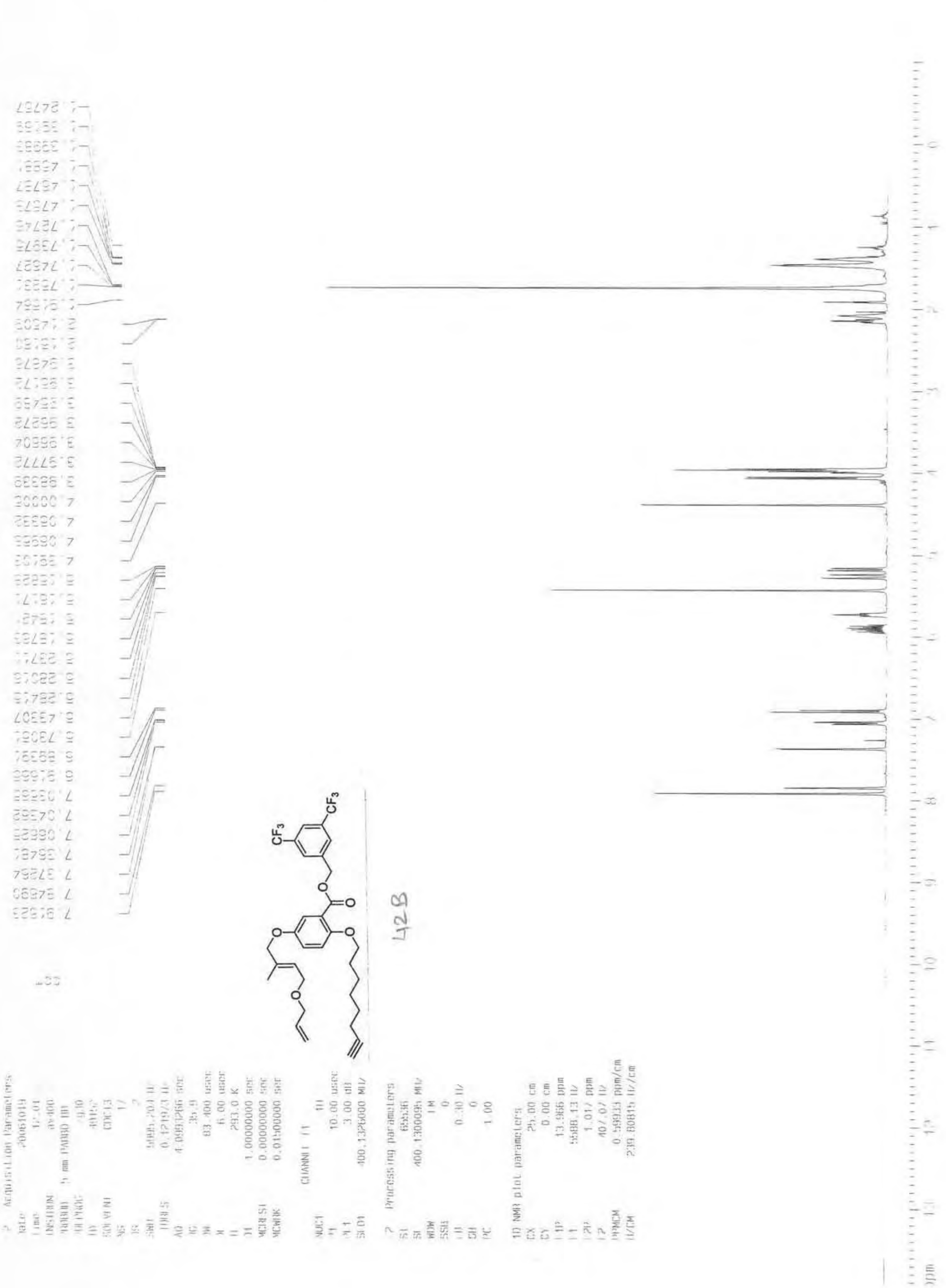

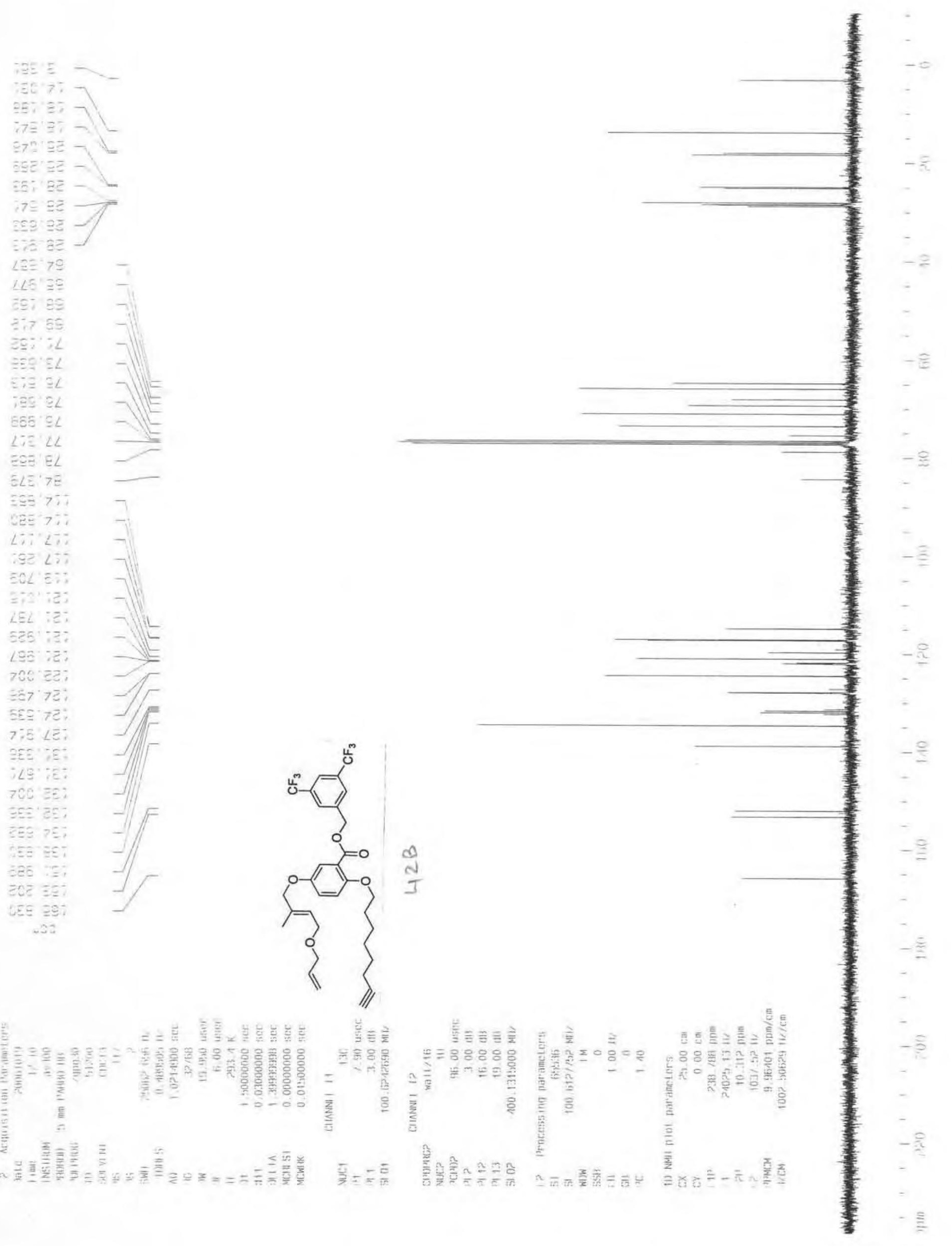


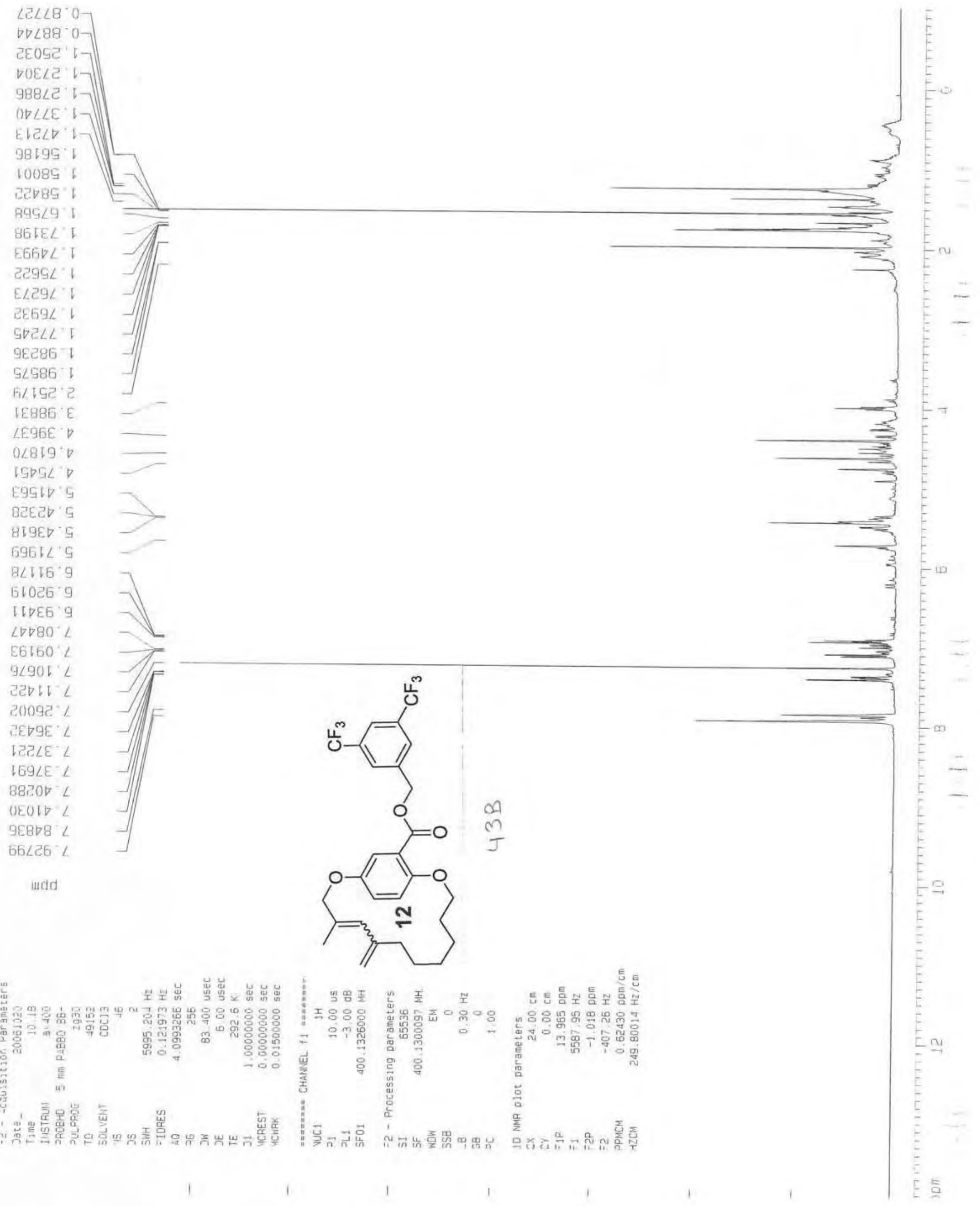




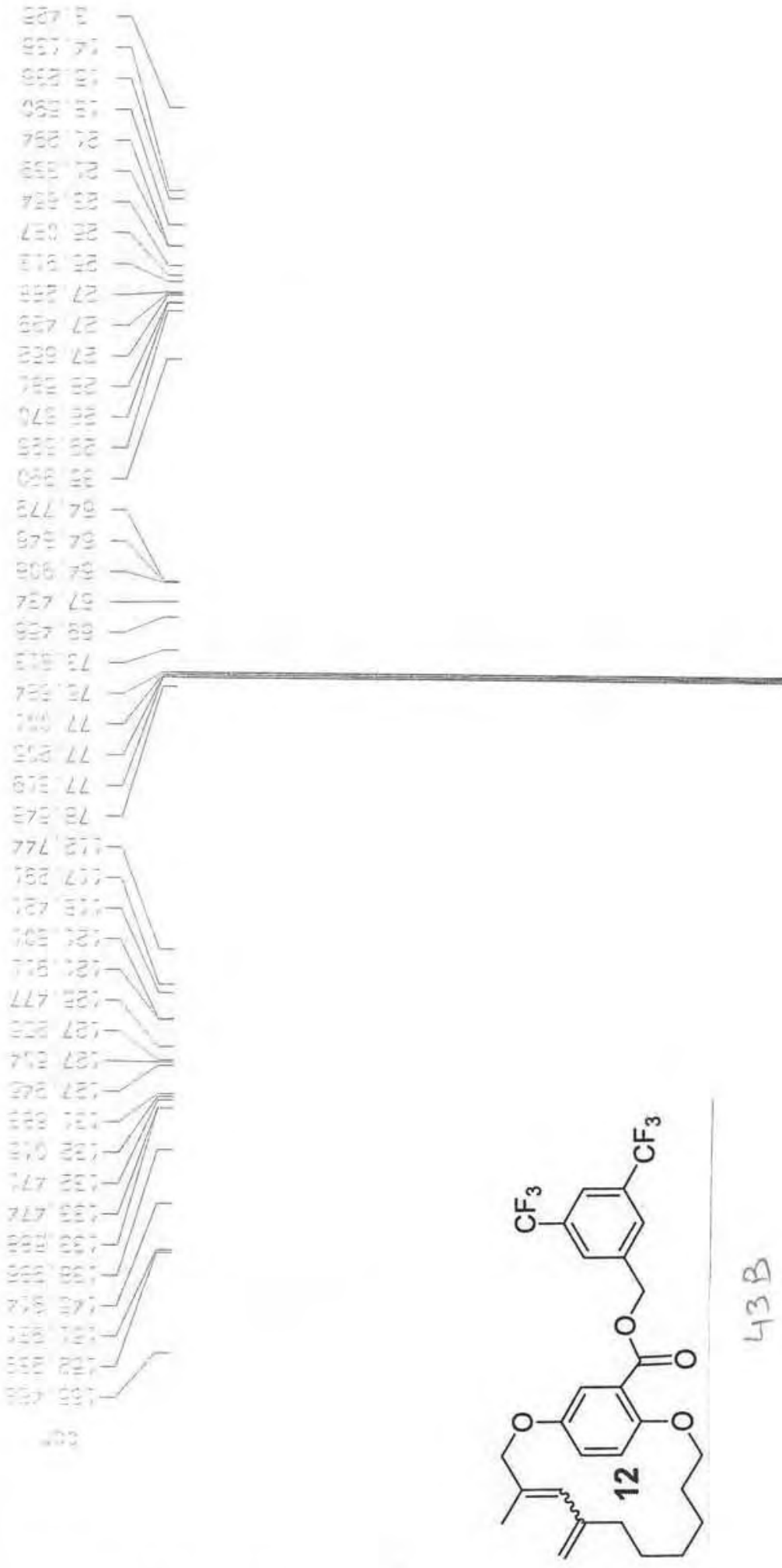

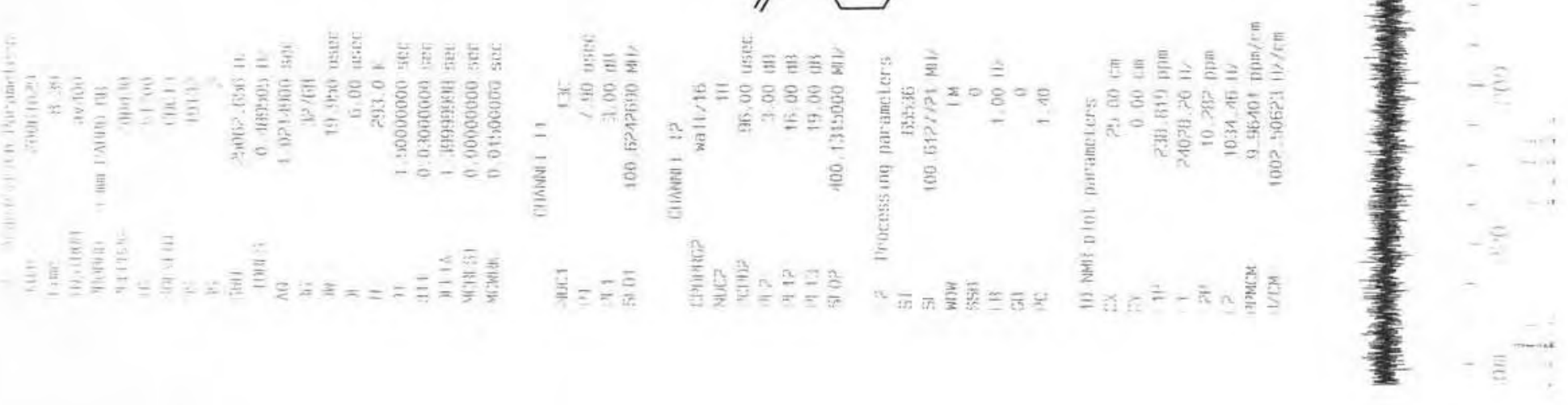



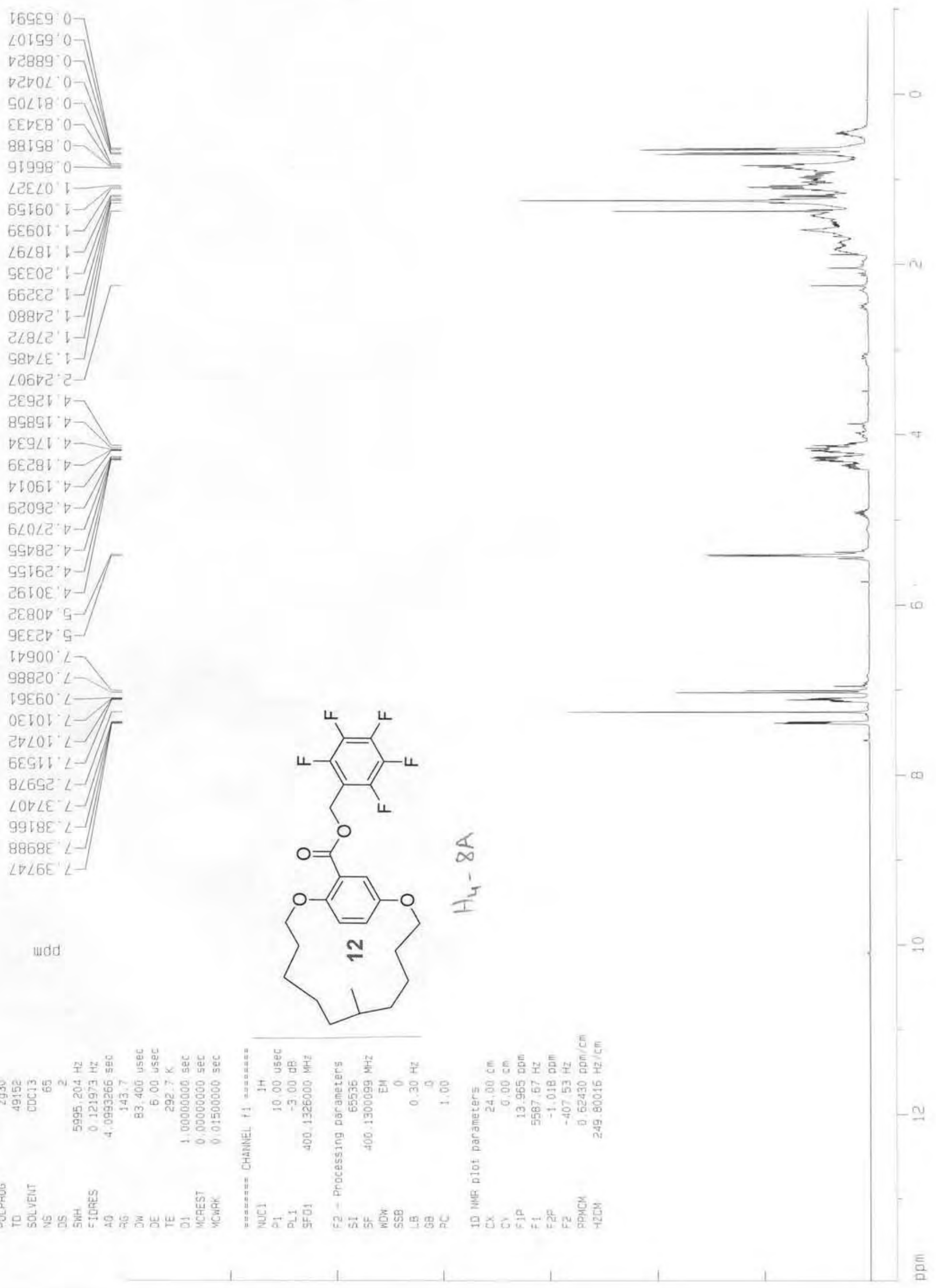


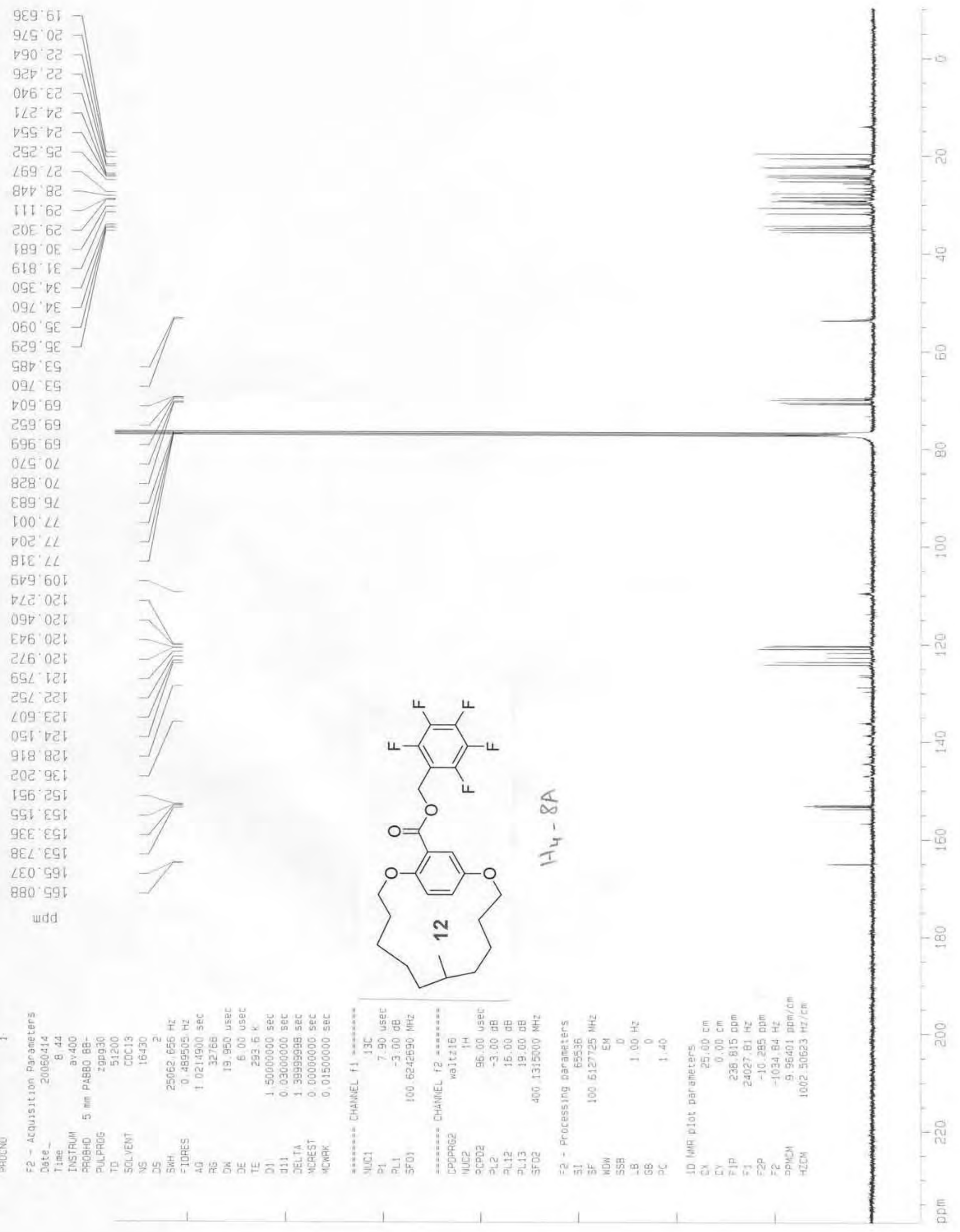



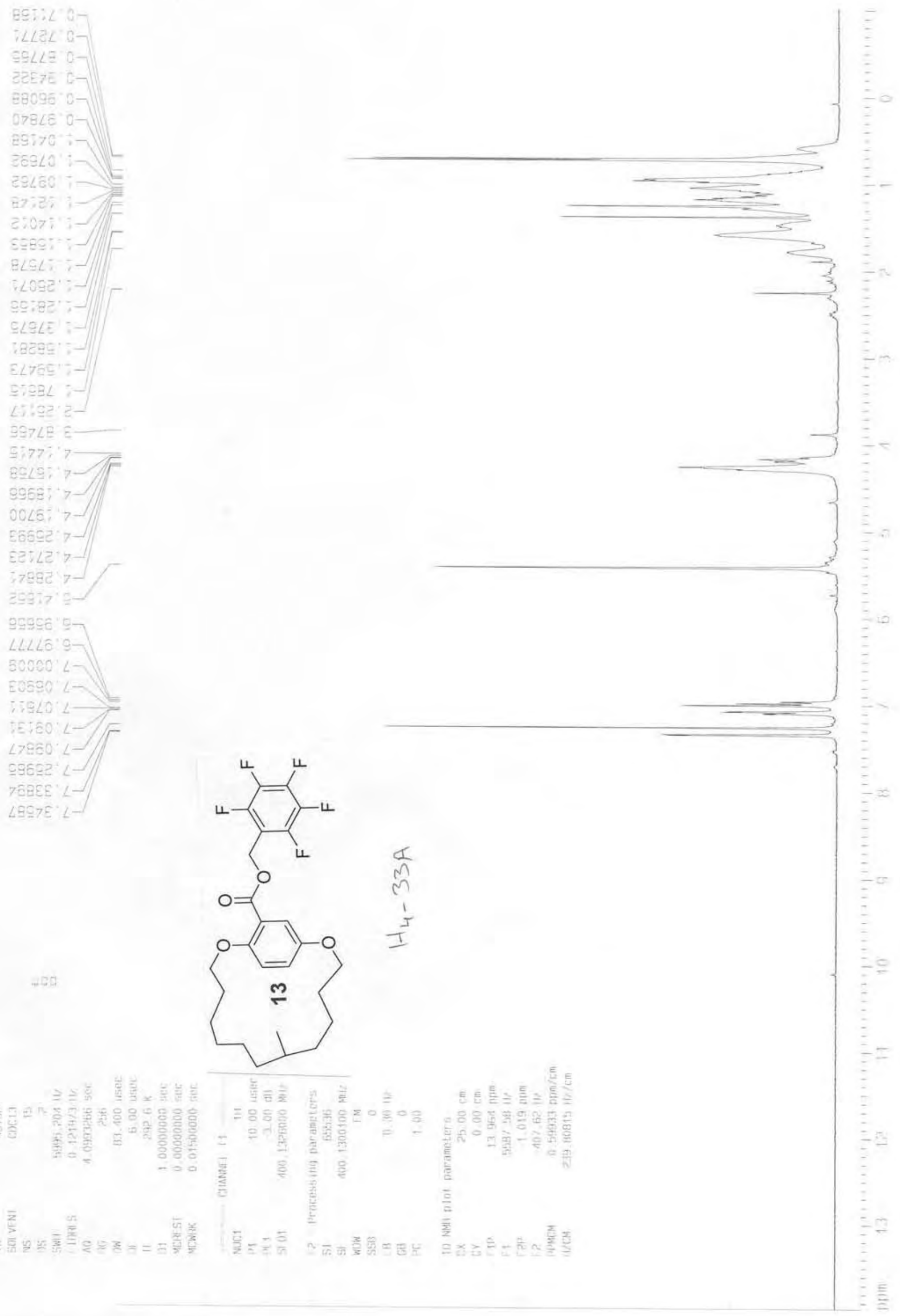

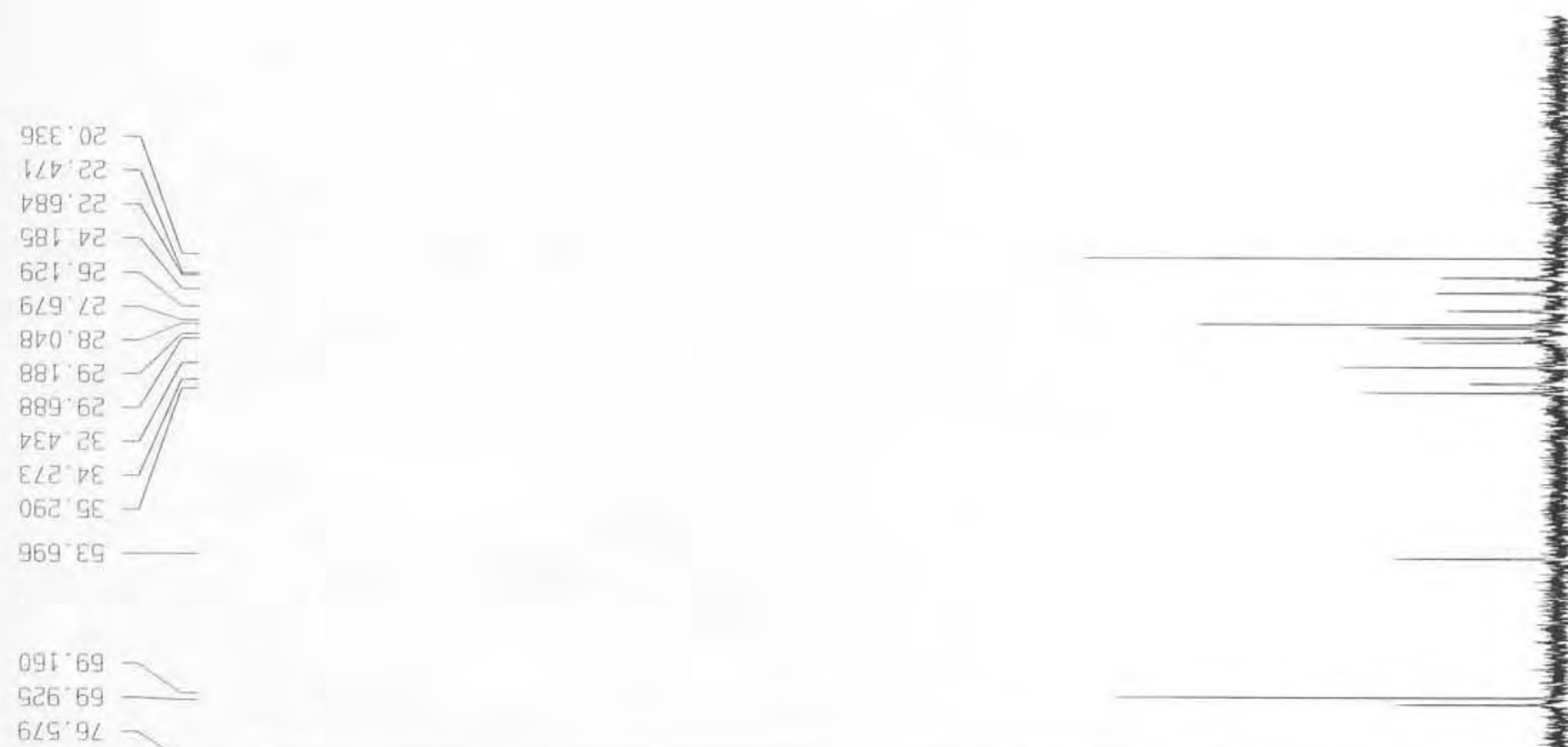

991' $69 \longrightarrow$

$6 \angle G^{\circ} 9 L$

$200^{\circ} \angle L$

$502 \%$

Gटb' L

\section{$10 \angle 501$} BEL'ZGI-
SEO'ESI$99 e^{\prime}$ ' 91 $\angle 80^{\circ}$ EZI-

992,491
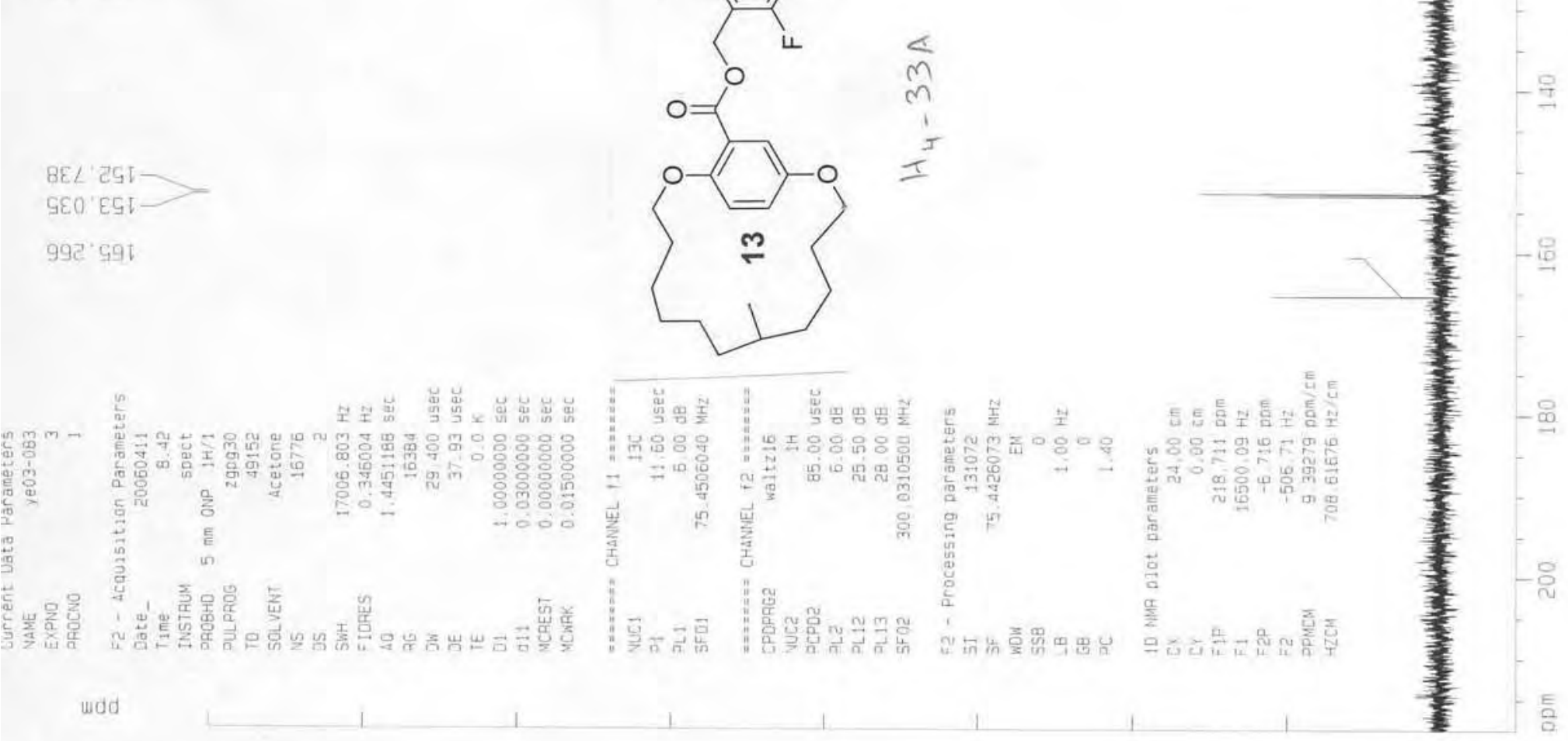


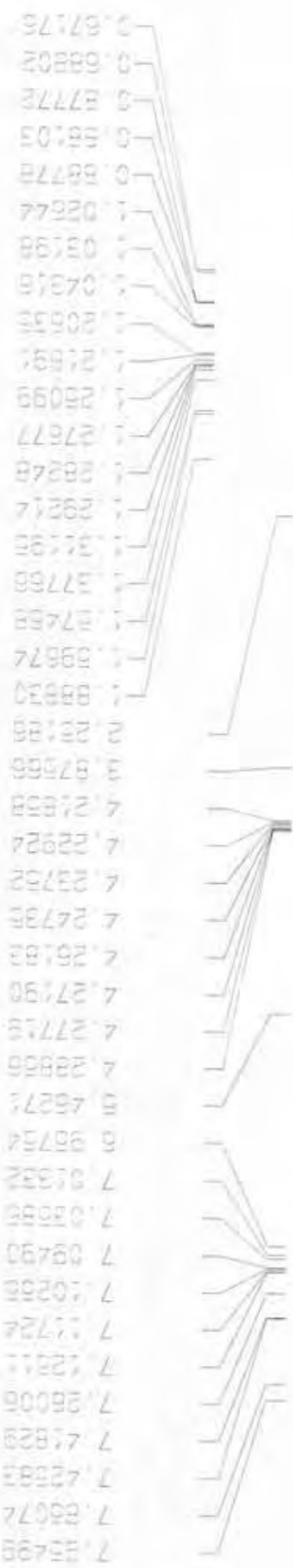

$4-35$

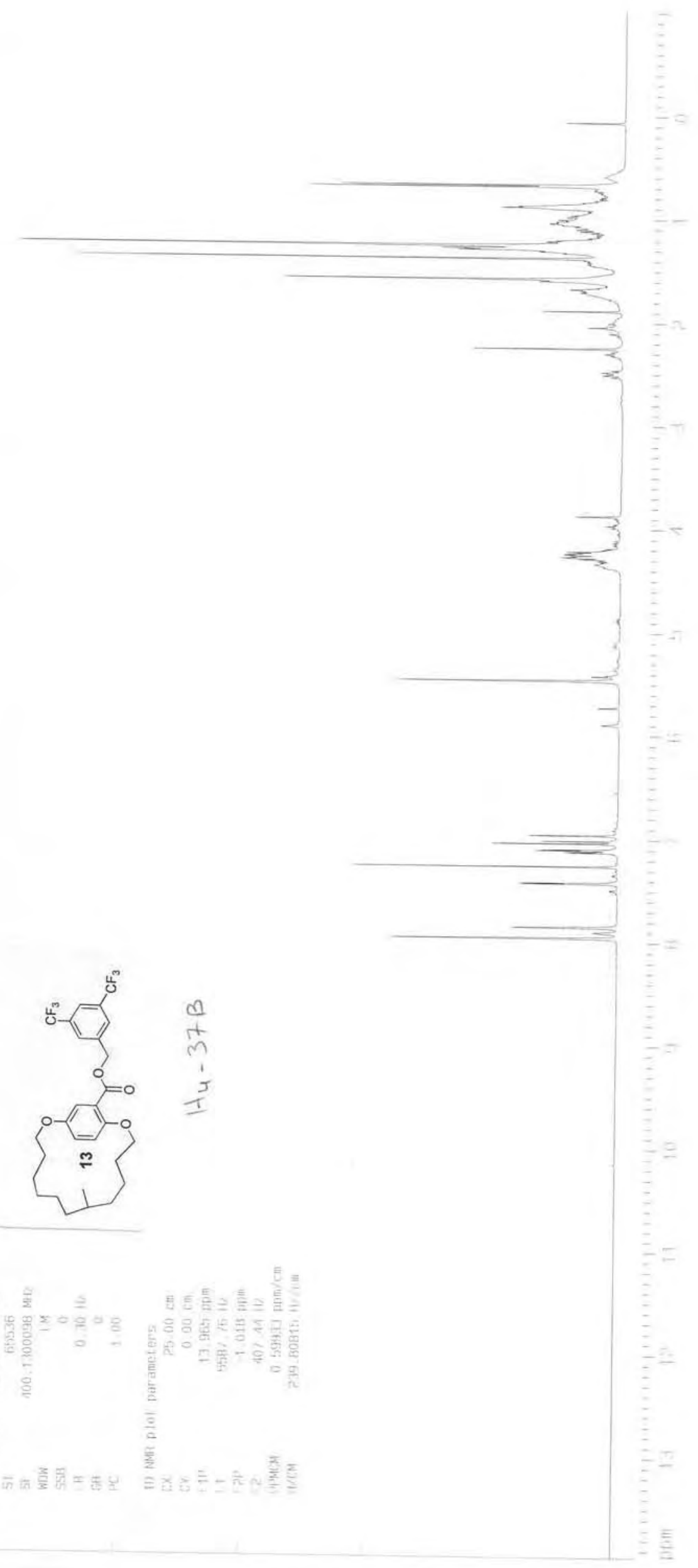




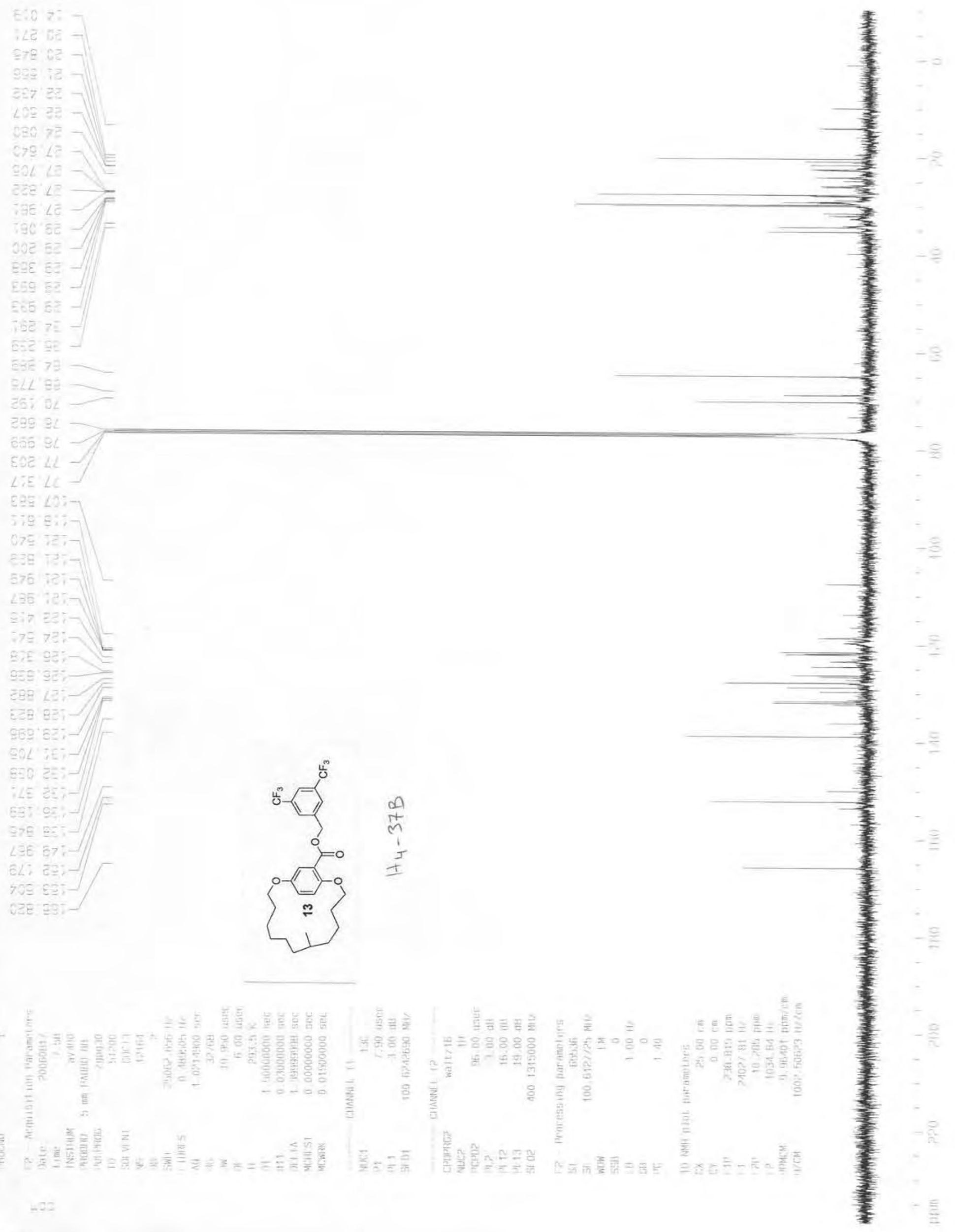

